\title{
A Systems Immunology Approach to Graft-Versus-Host Disease
}

Pedro Miguel de Ascensão Santos e Sousa

Thesis submitted to UCL in fulfilment of the requirements for the degree of Doctor of Philosophy

\author{
August 2016
}
Department of Haematology
UCL Cancer Institute
Royal Free Campus
Supervisors: Prof Ronjon Chakraverty
Prof Stephen Mackinnon
Dr Clare Bennett




\section{Declaration of originality}

I, Pedro Miguel de Ascensão Santos e Sousa confirm that the work presented in this thesis is my own. Where information has been derived from other sources, I confirm that this has been indicated in the thesis. 


\section{Abstract}

It is not known why only certain tissues are prone to graft-versus-host disease (GVHD) injury following allogeneic hematopoietic stem cell transplantation despite widespread antigen expression. Although it is known that $T$ cell effector pathways can have distinct effects upon individual GVHD organs, there has been no unbiased or systems-wide approach to defining the mechanisms underlying tissue-specific pathology. This thesis reports the results from a systems immunology approach to address the hypothesis that GVHD target tissues exert dominant, idiosyncratic roles in regulating effector T cell functions.

To test this concept, gene expression profiles of effector $\mathrm{CD} 8^{+} \mathrm{T}$ cells infiltrating lymphoid and GVHD target organs were compared in two clinically relevant murine GVHD models. Using Weighted Gene Network Correlation Analysis, a dichotomy between the transcriptomes of $\mathrm{T}$ cells in peripheral tissues and lymphoid organs was identified. These profiles diverged sharply between the different GVHD target organs, and between individual subcompartments of single organs, independently of the TCR repertoire and antigen distribution.

In the skin, expression of a broad effector program was determined by the transition of $\mathrm{T}$ cells from the dermis to the epidermis, in a process regulated by Langerhans cells (LC). In the absence of LC, T cells were rendered incapable of up-regulating the full panoply of effector genes, showed impaired 
differentiation into resident memory cells and failed to induce cutaneous GVHD. By performing localized LC depletion, it was demonstrated that LC regulated T cell effector programs in situ within the epidermis by providing signals to enhance local cytokine production, promote resistance to apoptosis and enhance local survival.

Collectively, these data demonstrate that GVHD is defined by tissueautonomous regulation of effector T cells; in the skin, this is dictated by interaction with epidermal LC in situ. This work provides a rationale for precision therapies directed at blocking GVHD in individual tissues. 


\section{Table of Contents}

$\begin{array}{ll}\text { List of Figures } & 10\end{array}$

$\begin{array}{ll}\text { List of Tables } & 17\end{array}$

$\begin{array}{ll}\text { List of abbreviations } & 19\end{array}$

I. INTRODUCTION 23

1. Haematopoietic stem-cell transplantation 24

1.1. Brief historical review 32

2. Graft-versus-host disease 40

2.1. Classification and clinical features 42

2.2. Acute GVHD immunopathology 45

a) Priming of the immune response $\quad 45$

b) Activation of donor T cells $\quad 48$

c) Alloreactive T cell proliferation and differentiation 51

d) Migration of effector cells to GVHD target tissues 52

e) Target tissue destruction by $T_{\text {eff }}$ cells $\quad 54$

$\begin{array}{lll}\text { 3. Pre-clinical models of GVHD } & 57\end{array}$

$\begin{array}{ll}\text { 3.1. Murine models } & 60\end{array}$

$\begin{array}{ll}\text { 4. Unanswered questions in GVHD } & 67\end{array}$

$\begin{array}{lll}\text { 5. Project rationale } & 69\end{array}$

$\begin{array}{ll}\text { 6. Hypothesis } & 72\end{array}$

$\begin{array}{ll}\text { 7. Aims and objectives } & \mathbf{7 2}\end{array}$ 
$\begin{array}{ll}\text { 1. Animal work } & \mathbf{7 4}\end{array}$

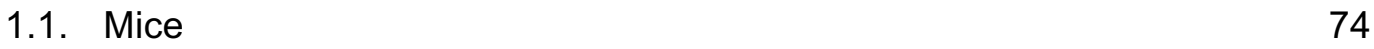

$\begin{array}{ll}\text { 1.2. Haematopoietic stem cell transplantation } & 74\end{array}$

$\begin{array}{ll}\text { 1.3. In vitro T cell activation } & 77\end{array}$

$\begin{array}{ll}\text { 1.4. Animal welfare } & 77\end{array}$

$\begin{array}{ll}\text { 1.5. Treatments } & 79\end{array}$

$\begin{array}{ll}\text { a) Antibiotic prophylaxis } & 79\end{array}$

b) Lymph node lymphocyte egress blockage $\quad 79$

$\begin{array}{ll}\text { c) Langerhans cell depletion } & 79\end{array}$

$\begin{array}{ll}\text { d) Notch signalling blockage } & 80\end{array}$

2. Tissue and organ harvest $\quad \mathbf{8 0}$

2.1. Isolation of murine immune cells $\quad 81$

a) Lymph nodes and spleens $\quad 81$

$\begin{array}{ll}\text { b) Bone marrow } & 81\end{array}$

c) Blood $\quad 82$

d) Liver $\quad 82$

e) Small intestine $\quad 82$

f) Skin $\quad 83$

3. Cell counting 84

$\begin{array}{lrl}\text { 4. Immunolabelling } & 84\end{array}$

$\begin{array}{ll}\text { 5. Flow cytometry } & \mathbf{8 6}\end{array}$

$\begin{array}{ll}\text { 6. Sample preparation for gene expression } & 87\end{array}$ 
$\begin{array}{ll}\text { 7. Microarray analysis } & 88\end{array}$

7.1. Data acquisition and processing 88

7.2. Samples relationship visualisation $\quad 89$

$\begin{array}{ll}\text { 7.3. Differential gene expression } & 89\end{array}$

7.4. Gene set enrichment analysis (GSEA) 90

7.5. Weighted gene co-expression network analysis (WGCNA) 90

$\begin{array}{ll}\text { 7.6. Gene ontology (GO) overrepresentation analysis } & 91\end{array}$

8. RNA-seq analysis 92

$\begin{array}{ll}\text { 8.1. Library preprocessing } & 92\end{array}$

8.2. Calculation of read-level metrics 92

8.3. Single-sample GSEA $\quad 92$

9. Statistical analysis 93

\begin{tabular}{lr} 
III. RESULTS & 95 \\
\hline
\end{tabular}

1. Transcription profiles of donor-derived $T_{\text {eff }}$ cells are different between SLO and GVHD target organs $\quad 96$

1.1. Brief characterization of the minor histocompatibility antigen mismatched $\begin{array}{ll}\text { models } & 98\end{array}$

a) $\mathrm{B} 6 \rightarrow 129$ model $\quad 98$

b) MataHari T cell model $\quad 99$

1.2. Purification and microarray analysis of donor derived $C D 8^{+} \mathrm{T}$ cells 107

$\begin{array}{ll}\text { 1.3. Exploratory data analysis } & 111\end{array}$

a) $\mathrm{B} 6 \rightarrow 129$ model $\quad 111$

b) MataHari T cell model 113 
1.4. SLO - GVHD target organ dichotomy reflects disparities in differentiation status of donor $\mathrm{T}_{\text {eff }}$ cells

1.5. Transcriptome diversity is neither due to early trafficking of effectors to the peripheral tissues nor to differential homing imprinting of donor-derived T cells 128

1.6. Discussion

2. $T_{\text {eff }}$ cell transcription profiles show compartmental specificity in acute

GVHD

2.1. Identification of gene clusters based on correlation and co-expression across tissues using WGCNA

2.2. WGCNA defined modules are preserved in an independent murine model of GVHD 147

2.3. Organ specific modules are conserved across species 149

2.4. Module annotation reveals biologically meaningful clusters of genes 156

2.5. Identification of highly connected hub genes 158

2.6. Discussion

3. Donor $\mathrm{CD}^{+} \mathrm{T}_{\text {eff }}$ pathogenicity in the skin is regulated in situ by epidermal Langerhans cells

3.1. LC depletion reduces the differences between dermal and epidermal donor $\mathrm{CD}^{+} \mathrm{T}_{\text {eff }}$ cells transcriptional profiles

3.2. In the absence of LC, epidermal donor T cells fail to fully up-regulate the effector cell programs

3.3. Donor $\mathrm{CD}^{+} \mathrm{T}$ cells expression of the epidermal specific transcriptional signature is regulated by host LC 
3.4. LC dictate donor $\mathrm{T}$ cell accumulation, survival, effector function and $\mathrm{T}_{\mathrm{RM}}$ differentiation potential in the epidermis

3.5. Responsiveness to INF-y by LC and Notch pathway signalling are central in the host LC - donor T cell crosstalk $\quad 188$

3.6. Discussion

1.1 Systems immunology - a comprehensive approach to acute GVHD study

1.2 A modular conception of the cellular programs underpinning GVHD development 208

1.3 A "two-hit" model of cutaneous acute GVHD development

1.4 Study limitations \& Future work 


\section{List of Figures}

Figure 1. Partial spectrum of conditioning regimens of various intensities and their impact on toxicity, and the dependence of transplant success upon GVT effects.

Figure 2. Timeline showing the main milestones in haematopoietic stem cell transplantation, 1957-2016.

Figure 3. Causes of death after HLA-matched sibling and unrelated donor HSCT.

Figure 4. Progression of events in aGvHD development.

Figure 5. Critical interactions for induction of GVHD.

Figure 6. Antigen presentation in GVHD in MHC-matched allogeneic stem-cell transplantation.

Figure 7. Genomic map of the mouse MHC complex.

Figure 8. B6 $\rightarrow 129$ model.

Figure 9. MataHari T cell model.

Figure 10. Adapted MataHari T cell $\rightarrow$ Langerin-DTR model.

Figure 11. Characterisation of the $\mathrm{B} 6 \rightarrow 129$ model. 
Figure 12. Donor $\mathrm{CD}^{+} \mathrm{T}$ cell infiltration of the secondary lymphoid organs and GVHD target organs at day 6 post-transplant.

Figure 13. Frequency of $\mathrm{H} 60$-specific from tissue infiltrating donor $\mathrm{CD}^{+} \mathrm{T}$ cells.

Figure 14. Kinetics of MataHari T cell infiltration of the GVHD target organs.

Figure 15. Characterisation of the MataHari T cell model.

Figure 16. Donor $\mathrm{CD}^{+}$(MataHari) T cell infiltration of the secondary lymphoid organs and GVHD target organs at day 7 post-transplant.

Figure 17. Strategy for high-purity FACS sorting of donor $\mathrm{CD}^{+} \mathrm{T}$ cells.

Figure 18. Staining strategy to isolate cells with equivalent number of divisions and homing imprinting.

Figure 19. Sort quality check and evaluation of sample purity.

Figure 20. Principal components analysis of the $B 6 \rightarrow 129$ data set.

Figure 21. Hierarchical clustering of the $B 6 \rightarrow 129$ data set, validated through non-parametric bootstrap methodology.

Figure 22. Multidimensional scaling analysis of the alloBMT samples from the $B 6 \rightarrow 129$ data set.

Figure 23. Hierarchical clustering of the MataHari $\mathrm{T}$ cell data set, validated through non-parametric bootstrap methodology.

Figure 24. Multidimensional scaling analysis of the alloBMT samples from the MataHari T cell data set. 
Figure 25. Transcriptional profiles are independent of the TCR repertoire and do not reflect antigen distribution.

Figure 26. Transcriptional differences between SLO and GVHD target organs are biologically meaningful.

Figure 27. Venn diagram representing the overlap of enriched gene sets from the KEGG PATHWAY collection in the GVHD target organs vs the SLO, found in the $\mathrm{B} 6 \rightarrow 129$ and the MataHari T cell models.

Figure 28. Heat map of selected genes commonly used to characterise $\mathrm{T}$ cell differentiation, including: transcription factors, chemokine/cytokine receptors, signalling molecules, integrins, activation markers, coregulatory molecules, and effector function molecules.

Figure 29. Comparison of the differentiation status of donor $\mathrm{CD}^{+} \mathrm{T}$ cells across the tissue. Single-sample GSEA was used to determine the enrichment for gene sets associated with Tc1/Tc17 polarisation, pathogenicity, and cytokine or effector function.

Figure 30. Experimental design to evaluate the effect of SLO lymphocyte egress blockage on the transcriptional profile of donor $\mathrm{CD} 8^{+} \mathrm{T}_{\text {eff }}$ cells in the lymph nodes.

Figure 31. Treatment with FTY720 blocks lymphocyte egress from the SLO.

Figure 32. Differences in gene expression between $T_{\text {eff }}$ cells are not due to early egress of optimally activated $\mathrm{T}_{\text {eff }}$ cells. 
Figure 33. Diversity of transcription profiles is not explained by differences in homing imprinting of donor T cells.

Figure 34. Selection of the power $\beta$ for gene correlation network generation.

Figure 35. Dendrogram obtained by hierarchical clustering of genes based on topological overlap, showing the assigned modules colour-coded.

Figure 36. Visualization of the eigengene network representing the relationships among the modules.

Figure 37. Heat map representation of the module-trait relationships.

Figure 38. Eigengene network generated using the edge-weighted spring embedded layout algorithm.

Figure 39. Module preservation study.

Figure 40. Validation of the murine modules on a data set from rhesus macaque.

Figure 41. Validation of the murine modules on a data set from human samples.

Figure 42. GSEA revealed statistically significant enrichment for module 24 in the samples from patients without GVHD.

Figure 43. GVHD target organ-associated modules accurately distinguish between samples isolated from the epidermis and samples isolated from the blood of transplanted patients with cutaneous acute GVHD. 
Figure 44. Immunofluorescence staining of the skin of alloBMT recipients with cutaneous acute GVHD (MataHari T cell model).

Figure 45. Host-derived LC replacement kinetics after alloBMT.

Figure 46. Effect of systemic host LC depletion on cutaneous acute GVHD.

Figure 47. Effect of localised host $\mathrm{LC}$ depletion on donor $\mathrm{CD} 8^{+} \mathrm{T}$ cell infiltration of the epidermis.

Figure 48. Correlation matrix comparing the transcription profiles of donor $\mathrm{CD}^{+} \mathrm{T}_{\text {eff }}$ cells isolated from the lymph nodes, dermis and epidermis of LC depleted and LC replete alloBMT recipients, at D+7 post-tranplant.

Figure 49. Heat map showing the enrichment for the 91 gene sets significantly over- or under-represented in the dermis vs the lymph nodes and in the epidermis $v s$ the dermis, with or without LC.

Figure 50. Classification of the gene sets that were differentially enriched in the epidermis in the presence of LC according to KEGG PATHWAY mapping.

Figure 51. Heat map of the 443 genes that form module 28 , comparing the transcriptional profiles of the lymph nodes, dermis and epidermis, in the presence or absence of LC.

Figure 52. Module 28 top 100 gene network, where the circles represent the genes and the lines represent the connectivity between the genes. 
Figure 53. Experimental design to evaluate the effect of LC depletion on proliferation, effector function and survival of skin infiltrating donor $\mathrm{CD} 8^{+} \mathrm{T}$ cells.

Figure 54. Assessment of the proliferation, survival and effector function of donor $\mathrm{CD}^{+} \mathrm{T}$ cells by FACS staining.

Figure 55. Summary data referring to the study of the effect of LC depletion upon proliferation, survival and effector function of donor $\mathrm{CD}^{+} \mathrm{T}_{\text {eff }}$ cells.

Figure 56. Experimental design to evaluate the effect of LC depletion donor $\mathrm{CD}^{+} \mathrm{T}$ cells capacity to differentiate into epidermal $\mathrm{T}_{\mathrm{RM}}$ cells.

Figure 57. Kinetics of donor derived $\mathrm{CD}^{+}$epidermal $\mathrm{T}_{\mathrm{RM}}$ cells in the presence or absence of LC.

Figure 58. Experimental design to evaluate the requirement of LC responsiveness to IFN- $\mathrm{Y}$ for donor $\mathrm{CD}^{+} \mathrm{T}$ cell accumulation in the epidermis.

Figure 59. Comparison of alloBMT driven changes in LC expression of MHC class I H-2Db, CD40, CD70 and CD86.

Figure 60. Study of the survival and effector function of donor $\mathrm{CD}^{+} \mathrm{T}$ cells isolated from alloBMT recipients incapable of detecting IFN-y signalling (lfngr1 $\left.{ }^{-1-}\right)$ vs WT.

Figure 61. Summary data refering to the study of the effect of LC responsiveness to IFN-Y signalling upon accumulation, survival and effector function of donor $\mathrm{CD} 8^{+} \mathrm{T}_{\text {eff }}$ cells. 
Figure 62. Experimental design to evaluate the effect of Notch signalling blockade on donor $\mathrm{CD}^{+} \mathrm{T}$ cell effector function in the epidermis.

Figure 63. Study of the effect of Notch signalling blockade on donor $\mathrm{CD}^{+} \mathrm{T}$ cell effector function in the spleen, dermis and epidermis. 


\section{List of Tables}

Table 1. Comparison of the characteristics of the 3 different sources of stem cells.

Table 2. Main clinical indications for HSCT in adult patients.

Table 3. Distinguishing acute and chronic GVHD.

Table 4. Organ involvement in acute GVHD.

Table 5. Organ involvement in chronic GVHD.

Table 6. Advantages and disadvantages of various preclinical model systems.

Table 7. H-2 haplotypes and alloantigens of some of the most commonly used laboratory mouse strains.

Table 8. Commonly used mouse models of HSCT.

Table 9. Assessment of clinical GVHD severity in transplanted subjects.

Table 10. Antibodies used for cell surface immunolabelling.

Table 11. Antibodies used for intracellular immunolabelling.

Table 12. Top 10 pathways enriched in GVHD target organs vs SLO common to both data sets (MSigDB C2:CP:KEGG collection). 
Table 13. WGCNA modules.

Table 14. Module specific driver genes summary.

Table 15. Top 10 type I and type II IFN responsive genes upregulated by LC after alloBMT. 


\section{List of abbreviations}

$\begin{array}{ll}\text { aGVHD } & \text { acute GVHD } \\ \text { allo } & \text { allogeneic } \\ \text { auto } & \text { autologous } \\ \text { APC } & \text { antigen presenting cells } \\ \text { AraC } & \text { cytosine arabinoside } \\ \text { ATG } & \text { anti-thymocyte globulin } \\ \text { BM } & \text { bone marrow } \\ \text { BMT } & \text { bone marrow transplantation } \\ \text { BSA } & \text { body surface area } \\ \text { BU } & \text { busulphan } \\ \text { CB } & \text { cord blood } \\ \text { cGVHD } & \text { chronic GVHD } \\ \text { CMV } & \text { cytomegalovirus } \\ \text { ConA } & \text { concanavalin A } \\ \text { CSA } & \text { cyclosporine A } \\ \text { CTL } & \text { cytotoxic T cells } \\ \text { CY } & \text { cyclophosphamide } \\ \text { DAMP } & \text { damage-associated molecular patterns } \\ \text { DE } & \text { differential expression } \\ \text { DETC } & \text { dendritic epidermal yo T cells } \\ \text { DLI } & \text { donor lymocyte infusion } \\ \text { DM } & \text { a }\end{array}$


DOT $\quad \mathrm{V} \delta 1^{+} \mathrm{T}$ cells

DT diphtheria toxin

EdU 5-ethynyl-2'-deoxyuridine

FACS Fluorescence-activated cell sorting

FLU fludarabine

FTY720 fingolimod

GO Gene Ontology

GSEA gene set enrichment analysis

GVHD graft-versus-host disease

GVL graft-versus-leukaemia

GVT graft-versus-tumour

HLA human leukocyte antigen

Hp haploidentical donor

HSCT haematopoietic stem cell transplantation

IEL intraepithelial lymphocytes

ILC3 group 3 innate lymphoid cells

KEGG Kyoto Encyclopedia of Genes and Genomes

LC Langerhans cells

LFA lymphocyte function-associated antigen

LN lymph nodes

LP lamina propria

MDS multidimensional scaling

MHC major histocompatibility complex

miHA minor histocompatibility antigen

MLN mesenteric lymph nodes

MSigDB Molecular Signatures Database 


\begin{tabular}{ll} 
MUD & matched unrelated donor \\
NES & normalised enrichment score \\
PBSC & peripheral blood stem cells \\
PCA & principal components analysis \\
PLN & peripheral lymph nodes \\
RIC & reduced intensity conditioning \\
S1P & sphingosine 1-phosphate \\
SIB & sibling \\
SLO & secondary lymphoid organs \\
SPF & specific pathogen-free \\
SSGSEA & single-sample gene set enrichment analysis \\
syn & syngeneic \\
TBI & total body irradiation \\
TCR & T cell receptor \\
Teff & effector T cells \\
TOM & topological overlap matrix \\
TRM & tissue resident memory T cells \\
WGCNA & weighted gene co-expression network analysis \\
WT & wild-type \\
\hline
\end{tabular}




\section{Introduction}




\section{Haematopoietic stem-cell transplantation}

Hematopoietic cell transplantation (HSCT) includes a variety of procedures in which a patient receives an infusion of hematopoietic progenitor cells following a preparative regimen of chemo- and/or radiotherapy, and can be broadly classified into 3 subtypes based upon the hematopoietic cell donor:

- Autologous HSCT (autoHSCT) refers to the reinfusion of the patient's hematopoietic progenitor cells, collected from the patient prior to the administration of high-dose chemotherapy targeted at an underlying malignancy, and is performed to rescue the patient from long-lasting and profound pancytopenia;

- Syngeneic HSCT (synHSCT) refers to the use of hematopoietic progenitor cells obtained from an identical twin, offering the advantage of providing a graft that is free of tumor cells;

- Allogeneic HSCT (alloHSCT) refers to the use of hematopoietic progenitor cells collected from a related or unrelated donor, who may display variable degrees of immunologic disparity with the recipient, thus adding the potentially beneficial of an immunological reaction against the tumour.

Allogeneic haematopoietic stem cell transplantation (alloHSCT) has revolutionised the treatment of many haematological and non-haematological 
malignancies, not only by permitting the usage of more intensive chemo- and radiotherapy regimens, but also, and most importantly, by introducing adoptive cellular immunotherapy into the clinical practice. ${ }^{(1)}$ Initially regarded as a means of replacing an irreversibly damaged haematopoietic compartment and overcoming the radio- and chemotherapy dose limitation imposed by bone marrow toxicity, it was soon recognised that this technique had a second powerful therapeutic effect, the graft-versus-tumour (GVT) effect, in which the host's tumour cells are recognize as foreign and killed by the lymphocytes contained in the donor graft. ${ }^{(2)}$ However, this alloreactive immune response is not restricted to the tumour sites and, while useful in combating any residual disease, when it affects the host's healthy tissues it can cause serious lifethreatening damage, the graft-versus-host disease (GVHD).

The success of alloHSCT is thus based on three basic principles: (1) conditioning of the patient in order to "create space" for donor stem cells to access the host stem cell niches and for engraftment to occur; (2) long term disease control, either through the eradication of the malignant cells or by reconstituting the damaged environment; and (3) modulation of the immune system, to balance the beneficial GVT effect while avoiding the development of GVHD. ${ }^{(3)}$

The conditioning of the recipient is a critical element in HSCT, thus, the selection of the optimal preparative regimen for any given patient is dependent upon disease-related factors (i.e. diagnosis and remission status) and patient-related factors (e.g. age, donor availability, comorbid conditions). ${ }^{(4)}$ Traditionally the conditioning of the patients was achieved by delivering 
maximally tolerated doses of multiple chemotherapeutic agents with non-overlapping toxicities, with or without radiation. However, even though this high-dose myeloablative approach was very effective against most leukaemias and lymphomas, and was able to penetrate the sanctuary sites, the associated toxicity limited its use to young patients with a good performance status. ${ }^{(4)}$ The increasing evidence that the eradication of malignant disorders could be accomplished through the GVT effect led to the development of reducedintensity and nonmyeloablative regimens, making allogeneic HSCT a viable therapeutic option for frail and older and patients, unable to tolerate high-dose conditioning (Figure 1). ${ }^{(4,5)}$

Initially, the main graft source was the bone marrow, harvested from the posterior iliac crests under general anaesthesia (hence the designation of bone marrow transplantation, BMT). ${ }^{(6)}$ Further characterisation of the haematopoietic stem cell compartment prompted their identification in the peripheral blood, ${ }^{(7-9)}$ and the development of new techniques to enrich for and harvest them. ${ }^{(10)}$ Subsequently, mobilized peripheral blood stem cells (PBSC) have become one of the main stem cell source for HSCT, and although no standardised indications have been established to prefer PBSC over bone marrow, operating room unavailability, lack of personnel to perform bone marrow aspiration, contraindication to general anaesthesia and choice of the donor, are some of the factors that may determine this trend. ${ }^{(11)}$ The discovery of cord blood as a rich source of stem cells, in $1978,{ }^{(12)}$ and its successful use in allogeneic HSCT, ${ }^{(13)}$ provided a third source of stem cell 


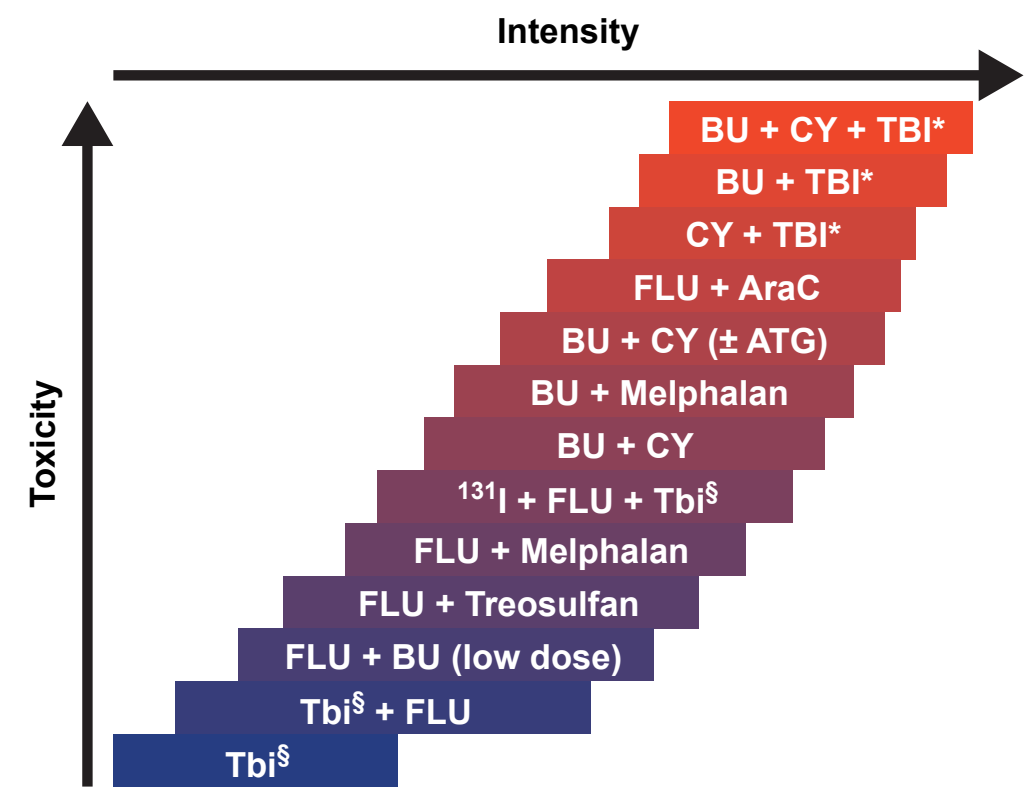

\section{Requirement of GVT effect}

Figure 1. Partial spectrum of conditioning regimens of various intensities and their impact on toxicity, and the dependence of transplant success upon GVT effects.

AraC, cytosine arabinoside; ATG, anti-thymocyte globulin; BU, busulfan; CY, cyclophosphamide; FLU, fludarabine; ${ }^{131} \mathrm{I}$, radioiodine; TBI, total body irradiation; *High-dose TBI (800-1320 cGy); ' Low-dose TBI (200-400 cGy).

Adapted with permission from Deeg HJ, Maris MB, Scott BL, Warren EH. Optimization of allogeneic transplant conditioning: Not the time for dogma. Leukemia 2006, 20(10):1701-5; Copyright Nature Publishing Group. 
devoid of risks to the donors, with reduced likelihood of transmitting infections, particularly CMV, which can be fully tested and HLA typed, cryopreserved, and made available for immediate use (Table 2). ${ }^{(6)}$

Moreover, manipulation of the cellular components of the grafts via $\mathrm{CD}^{+}{ }^{+}$and $\mathrm{T}$ cell depletion has expanded the field even further, extending the option of HSCT to patients without an HLA-matched related or unrelated donor. ${ }^{(14)}$ The success of this approach has fuelled the search for the optimal graft composition, providing rapid engraftment kinetics and enhanced immune reconstitution, with high antitumoural activity but low GVHD-inducing potential. The leading strategies already in clinical trials include infusion of regulatory $T$ cells, ${ }^{(15)}$ graft depletion of $\alpha \beta^{+} T$ and $B$ cells, ${ }^{(16,17)}$ NK cell adoptive immunotherapy, ${ }^{(18)}$ and selective allo-depletion of T cells. ${ }^{(19)}$

HSCT has thus evolved, in just a few decades, from an experimental procedure for patients with bone marrow failure or incurable leukaemia to a standard of care therapy for an expanding range of patients with congenital or acquired disorders of the hematopoietic system or radio-, chemo- or immunesensitive malignancies (Table 2). ${ }^{(20)}$ 
Table 1. Comparison of the characteristics of the 3 different sources of stem cells.

\begin{tabular}{|c|c|c|c|}
\hline & BM & PBSC & CB \\
\hline $\begin{array}{l}\text { Typical time frame from } \\
\text { initiation of search to } \\
\text { transplantation }\end{array}$ & 3-6 months & $3-6$ months & 2-4 weeks \\
\hline Adverse effects for donor & $\begin{array}{l}\text { wound infection, } \\
\text { bleeding, general } \\
\text { anesthesia, } \\
\text { etc. }\end{array}$ & $\begin{array}{c}\text { bleeding/thrombosis, } \\
\text { infection, hypotension, } \\
\text { electrolyte disturbance, } \\
\text { etc. }\end{array}$ & None \\
\hline $\begin{array}{l}\text { Minimal cell dose for } \\
\text { transplant }\end{array}$ & $\begin{array}{l}\text { Total nucleated cell: } \\
\qquad 2 \times 10^{8} / \mathrm{kg}\end{array}$ & $\begin{array}{l}\text { Total CD } 34^{+} \text {cell: } \\
2 \times 10^{6} / \mathrm{kg}\end{array}$ & $\begin{array}{c}\text { Total nucleated cell: } \\
2.5 \times 10^{7} / \mathrm{kg}\end{array}$ \\
\hline $\begin{array}{l}\text { Possibility to give additional } \\
\text { stem cell dose }\end{array}$ & Possible & Possible & Impossible \\
\hline HLA matching requirement & $\begin{array}{l}\text { More stringent } \\
(7-8 / 8 \text { matched })\end{array}$ & $\begin{array}{l}\text { More stringent } \\
(7-8 / 8 \text { matched })\end{array}$ & $\begin{array}{l}\text { Less stringent } \\
(4-6 / 6 \text { matched })\end{array}$ \\
\hline Neutrophil engraftment & About 3 weeks & About 2 weeks & About 4 weeks \\
\hline Immune reconstitution & Faster & Faster & Slower \\
\hline GVHD risk & Medium & Highest & Lowest \\
\hline Post-HSCT infection risk & Lower & Lower & Higher \\
\hline Latent virus transmission risk & Higher & Higher & Lower \\
\hline CMV transmission risk & Higher & Higher & Lower \\
\hline Relapse risk & Higher & Lower & Higher \\
\hline
\end{tabular}

BM, bone marrow; CB, cord blood; CMV, cytomegalovirus; GVHD, graft-versus-host disease; HLA: human leukocyte antigen; PBSC, peripheral blood stem cell. 
Table 2. Main clinical indications for HSCT in adult patients.

\begin{tabular}{|c|c|c|c|c|c|}
\hline Disease & Disease status & SIB $^{*}$ & MUD* & $\mathrm{CB} / \mathrm{Hp}^{*}$ & Auto* \\
\hline $\begin{array}{l}\text { Acute myeloid } \\
\text { leukaemia }\end{array}$ & $\begin{array}{l}\text { CR1 (low risk) } \\
\text { CR1 (intermediate) } \\
\text { CR1 (high risk) } \\
\text { CR2 } \\
\text { CR3, incipient relapse } \\
\text { M3 Molecular persistence } \\
\text { M3 Molecular CR2 } \\
\text { Relapse or refractory }\end{array}$ & $\begin{array}{l}\text { CO (II) } \\
\text { S (II) } \\
\text { S (II) } \\
\text { S (II) } \\
\text { S (III) } \\
\text { S (II) } \\
\text { S (II) } \\
\text { CO (II) }\end{array}$ & $\begin{array}{l}\text { D (II) } \\
\text { CO (II) } \\
\text { S (II) } \\
\text { S (II) } \\
\text { CO (III) } \\
\text { CO (II) } \\
\text { CO (II) } \\
\text { CO (II) }\end{array}$ & $\begin{array}{l}\text { GNR (II) } \\
\text { D (II) } \\
\text { CO (II) } \\
\text { CO (II) } \\
\text { D (III) } \\
\text { GNR (III) } \\
\text { GNR (III) } \\
\text { D (II) }\end{array}$ & $\begin{array}{l}\text { CO (I) } \\
\text { S (I) } \\
\text { CO (I) } \\
\text { CO (II) } \\
\text { GNR (III) } \\
\text { GNR (III) } \\
\text { S (II) } \\
\text { GNR (III) }\end{array}$ \\
\hline $\begin{array}{l}\text { Acute lymphoblastic } \\
\text { leukaemia }\end{array}$ & $\begin{array}{l}\mathrm{Ph}^{(-)}, \mathrm{CR} 1 \text { (standard risk) } \\
\mathrm{Ph}^{(-)}, \mathrm{CR} 1 \text { (high risk) } \\
\mathrm{Ph}^{(+)}, \mathrm{CR} 1 \\
\text { CR2, incipient relapse } \\
\text { Relapse or refractory }\end{array}$ & $\begin{array}{l}\text { D (II) } \\
\text { S (II) } \\
\text { S (II) } \\
\text { S (II) } \\
\text { CO (II) }\end{array}$ & $\begin{array}{l}\text { GNR (II) } \\
\text { S (II) } \\
\text { S (II) } \\
\text { S (II) } \\
\text { D (II) }\end{array}$ & $\begin{array}{l}\text { GNR (III) } \\
\text { CO (II) } \\
\text { CO (II) } \\
\text { CO (II) } \\
\text { D (II) }\end{array}$ & $\begin{array}{l}\text { CO (III) } \\
\text { GNR (III) } \\
\text { CO (III) } \\
\text { GNR (II) } \\
\text { GNR (III) }\end{array}$ \\
\hline $\begin{array}{l}\text { Chronic myeloid } \\
\text { leukaemia }\end{array}$ & $\begin{array}{l}\text { 1st CP, failing TKI } \\
\text { Accelerated phase or }>1 \mathrm{st} \mathrm{CP} \\
\text { Blast crisis }\end{array}$ & $\begin{array}{l}S \text { (II) } \\
\text { S (II) } \\
S \text { (II) }\end{array}$ & $\begin{array}{l}S \text { (II) } \\
\text { S (II) } \\
\text { S (II) }\end{array}$ & $\begin{array}{l}\mathrm{CO}(\mathrm{III}) \\
\mathrm{CO}(\mathrm{II}) \\
\mathrm{CO}(\mathrm{II})\end{array}$ & $\begin{array}{l}\text { GNR (II) } \\
\text { D (III) } \\
\text { GNR (III) }\end{array}$ \\
\hline Myelofibrosis & $\begin{array}{l}\text { Primary or secondary with an int. } \\
\text { or high DIPSS score }\end{array}$ & $S(I I)$ & $S(I I)$ & $S(I I I)$ & GNR (III) \\
\hline $\begin{array}{l}\text { Myelodysplastic } \\
\text { syndromes }\end{array}$ & $\begin{array}{l}\text { RA, RCMD, RAEB I and II } \\
\text { sAML in CR1 or CR2 } \\
\text { More advanced stages }\end{array}$ & $\begin{array}{l}S(I I) \\
S(I I) \\
S(I I)\end{array}$ & $\begin{array}{l}S(I I) \\
S \text { (II) } \\
S \text { (II) }\end{array}$ & $\begin{array}{l}S(I I) \\
S(I I) \\
S(I I)\end{array}$ & $\begin{array}{l}\text { GNR (III) } \\
\text { CO (II) } \\
\text { GNR (III) }\end{array}$ \\
\hline $\begin{array}{l}\text { Chronic lymphocytic } \\
\text { leukaemia }\end{array}$ & Poor risk disease & $S(I I)$ & $S(I I)$ & $\mathrm{D}(\mathrm{III})$ & GNR (I) \\
\hline $\begin{array}{l}\text { Diffuse large B cell } \\
\text { lymphoma }\end{array}$ & $\begin{array}{l}\text { CR1 (intermediate/high IPI at dx) } \\
\text { Chemosensitive relapse, } \geq \text { CR2 } \\
\text { Chemosensitive relapse after } \\
\text { autoHSCT failure } \\
\text { Refractory disease }\end{array}$ & $\begin{array}{l}\text { GNR (III) } \\
\text { CO (II) } \\
\mathrm{S}(\mathrm{II}) \\
\mathrm{CO}(\mathrm{II})\end{array}$ & $\begin{array}{l}\text { GNR (III) } \\
\text { CO (II) } \\
\text { S (II) } \\
\text { CO (II) }\end{array}$ & $\begin{array}{l}\text { GNR (III) } \\
\text { D (III) } \\
\text { CO (III) } \\
\text { D (III) }\end{array}$ & $\begin{array}{l}\text { CO (I) } \\
\text { S (I) } \\
\text { GNR (III) } \\
\text { CO (II) }\end{array}$ \\
\hline Mantle cell lymphoma & $\begin{array}{l}\text { CR1 } \\
\text { CR/PR }>1 \text {, no prior autoHSCT } \\
\text { CR/PR }>1 \text {, prior autoHSCT } \\
\text { Refractory }\end{array}$ & $\begin{array}{l}\text { D (III) } \\
\text { CO (III) } \\
\text { S (II) } \\
\text { CO (II) }\end{array}$ & $\begin{array}{l}\text { D (III) } \\
\text { CO (III) } \\
\text { S (II) } \\
\text { CO (II) }\end{array}$ & $\begin{array}{l}\text { GNR (III) } \\
\text { D (III) } \\
\text { CO (III) } \\
\text { D (III) }\end{array}$ & $\begin{array}{l}\text { S (I) } \\
\text { S (II) } \\
\text { GNR (II) } \\
\text { GNR (II) }\end{array}$ \\
\hline Follicular lymphoma & $\begin{array}{l}\text { CR1 } \\
\text { Chemosensitive relapse, } \geq \text { CR2 } \\
\geq \text { CR2 after auto-HSCT failure } \\
\text { Refractory }\end{array}$ & $\begin{array}{l}\text { GNR (III) } \\
\text { CO (III) } \\
\text { S (II) } \\
\text { CO (II) }\end{array}$ & $\begin{array}{l}\text { GNR (III) } \\
\text { CO (III) } \\
\text { S (II) } \\
\text { CO (II) }\end{array}$ & $\begin{array}{l}\text { GNR (III) } \\
\text { GNR (III) } \\
\text { D (III) } \\
\text { CO (III) }\end{array}$ & $\begin{array}{l}\text { D (II) } \\
\text { S (II) } \\
\text { GNR (III) } \\
\text { GNR (III) }\end{array}$ \\
\hline $\begin{array}{l}\text { Waldenstrom } \\
\text { macroglobulinaemia }\end{array}$ & $\begin{array}{l}\text { CR1 } \\
\text { Chemosensitive relapse, } \geq \mathrm{CR} 2 \\
\text { Poor risk disease }\end{array}$ & $\begin{array}{l}\text { GNR (III) } \\
\text { GNR (III) } \\
\text { CO (II) }\end{array}$ & $\begin{array}{l}\text { GNR (III) } \\
\text { GNR (III) } \\
\text { CO (II) }\end{array}$ & $\begin{array}{l}\text { GNR (III) } \\
\text { GNR (III) } \\
\text { D (III) }\end{array}$ & $\begin{array}{l}\text { D (II) } \\
\text { CO (II) } \\
\text { GNR (III) }\end{array}$ \\
\hline T cell lymphoma & $\begin{array}{l}\text { CR1 } \\
\text { Chemosensitive relapse, } \geq \text { CR2 } \\
\text { Refractory }\end{array}$ & $\begin{array}{l}\mathrm{CO}(\mathrm{II}) \\
\mathrm{S}(\mathrm{II}) \\
\mathrm{CO}(\mathrm{II})\end{array}$ & $\begin{array}{l}\mathrm{CO}(\mathrm{II}) \\
\mathrm{S}(\mathrm{II}) \\
\mathrm{CO}(\mathrm{II})\end{array}$ & $\begin{array}{l}\text { GNR (III) } \\
\text { CO (III) } \\
\text { CO (III) }\end{array}$ & $\begin{array}{l}\text { CO (II) } \\
\text { CO (II) } \\
\text { GNR (II) }\end{array}$ \\
\hline $\begin{array}{l}\text { Primary cutaneous T } \\
\text { cell lymphoma }\end{array}$ & $\begin{array}{l}\text { EORTC/ISCL I-IIA (early) } \\
\text { EORTC/ISCL IIB-IV (advanced) }\end{array}$ & $\begin{array}{l}\text { GNR (III) } \\
\text { CO (III) }\end{array}$ & $\begin{array}{l}\text { GNR (III) } \\
\text { CO (III) }\end{array}$ & $\begin{array}{l}\text { GNR (III) } \\
\text { D (III) }\end{array}$ & $\begin{array}{l}\text { GNR (III) } \\
\text { GNR (III) }\end{array}$ \\
\hline \multirow[t]{2}{*}{ Hodgkin's lymphoma } & $\begin{array}{l}\text { CR1 } \\
\text { Chemosensitive relapse, no prior } \\
\text { autoHSCT }\end{array}$ & $\begin{array}{l}\text { GNR (III) } \\
\text { D (III) }\end{array}$ & $\begin{array}{l}\text { GNR (III) } \\
\text { D (III) }\end{array}$ & $\begin{array}{l}\text { GNR (III) } \\
\text { GNR (III) }\end{array}$ & $\begin{array}{l}\text { GNR (I) } \\
\mathrm{S}(\mathrm{I})\end{array}$ \\
\hline & $\begin{array}{l}\text { Chemosensitive relapse, prior } \\
\text { autoHSCT } \\
\text { Refractory }\end{array}$ & $\begin{array}{l}S(I I) \\
D(I I)\end{array}$ & $\begin{array}{l}S(I I) \\
D(I I)\end{array}$ & $\begin{array}{l}\text { CO (III) } \\
\text { D (III) }\end{array}$ & $\begin{array}{l}\mathrm{CO}(\mathrm{III}) \\
\mathrm{CO}(\mathrm{III})\end{array}$ \\
\hline Multiple myeloma & & $\mathrm{CO}(\mathrm{I})$ & $\mathrm{CO}(\mathrm{II})$ & GNR (III) & $S(I)$ \\
\hline
\end{tabular}


Table 2. (continued)

\begin{tabular}{|c|c|c|c|c|c|}
\hline Disease & Disease status & SIB & MUD & CB/Hp & Auto \\
\hline Amyloidosis & & CO (III) & CO (III) & GNR (III) & $\mathrm{CO}(\mathrm{II})$ \\
\hline $\begin{array}{l}\text { Acquired severe } \\
\text { aplastic anaemia }\end{array}$ & $\begin{array}{l}\text { Newly diagnosed } \\
\text { Relapse/Refractory }\end{array}$ & $\begin{array}{l}S(I I) \\
S(I I)\end{array}$ & $\begin{array}{l}\mathrm{CO}(\mathrm{II}) \\
\mathrm{S}(\mathrm{II})\end{array}$ & $\begin{array}{l}\text { GNR (III) } \\
\text { CO (II) }\end{array}$ & $\begin{array}{l}\text { NA } \\
\text { NA }\end{array}$ \\
\hline \multirow{2}{*}{$\begin{array}{l}\text { Constitutional severe } \\
\text { aplastic anaemia }\end{array}$} & Fanconi anaemia & $S(I I)$ & S (II) & $\mathrm{CO}(\mathrm{II})$ & NA \\
\hline & Dyskeratosis congenital & $S(I I)$ & $S(I I)$ & $\mathrm{CO}(\mathrm{II})$ & NA \\
\hline $\begin{array}{l}\text { Acquired aplastic } \\
\text { anaemia/PNH }\end{array}$ & $\begin{array}{l}\text { Newly diagnosed } \\
\text { Relapse/Refractory }\end{array}$ & $\begin{array}{l}S(I I) \\
S(I I)\end{array}$ & $\begin{array}{l}\mathrm{CO}(\mathrm{II}) \\
\mathrm{S}(\mathrm{II})\end{array}$ & $\begin{array}{l}\text { GNR (III) } \\
\text { CO (II) }\end{array}$ & $\begin{array}{l}\text { NA } \\
\text { NA }\end{array}$ \\
\hline Haemolytic PNH & & GNR (II) & GNR (II) & GNR (II) & NA \\
\hline Breast cancer & $\begin{array}{l}\text { Adjuvant high risk, HER2-negative } \\
\text { Metastatic, chemosensitive }\end{array}$ & $\begin{array}{l}\text { GNR (III) } \\
\text { D (II) }\end{array}$ & $\begin{array}{l}\text { GNR (III) } \\
\text { D (II) }\end{array}$ & $\begin{array}{l}\text { GNR (III) } \\
\text { GNR (III) }\end{array}$ & $\begin{array}{l}\mathrm{CO}(\mathrm{II}) \\
\mathrm{D} / \mathrm{CO}(\mathrm{II})\end{array}$ \\
\hline Germ cell tumours & $\begin{array}{l}\text { Second line, high risk } \\
\text { Primary refractory, second \& further } \\
\text { relapse }\end{array}$ & $\begin{array}{l}\text { GNR (III) } \\
\text { GNR (III) }\end{array}$ & $\begin{array}{l}\text { GNR (III) } \\
\text { GNR (III) }\end{array}$ & $\begin{array}{l}\text { GNR (III) } \\
\text { GNR (III) }\end{array}$ & $\begin{array}{l}\mathrm{CO}(\mathrm{II}) \\
\mathrm{S}(\mathrm{II})\end{array}$ \\
\hline Ovarian cancer & High risk/recurrent & $\mathrm{D}(\mathrm{II})$ & GNR (III) & GNR (III) & GNR (I-II) \\
\hline Medulloblastoma & Post-surgery, high risk & GNR (III) & GNR (III) & GNR (III) & $\mathrm{D} / \mathrm{CO}(\mathrm{III})$ \\
\hline Small cell lung cancer & Limited & GNR (III) & GNR (III) & GNR (III) & $D(I-I I)$ \\
\hline Soft tissue sarcoma & Metastatic & $\mathrm{D}(\mathrm{III})$ & GNR (III) & GNR (III) & GNR (II) \\
\hline $\begin{array}{l}\text { Ewing's sarcoma } \\
\text { family of tumours }\end{array}$ & $\begin{array}{l}\text { Locally advanced/metastatic, } \\
\text { chemosensitive }\end{array}$ & $\mathrm{D}(\mathrm{III})$ & GNR (III) & GNR (III) & $\mathrm{CO}(\mathrm{III})$ \\
\hline Renal cell carcinoma & Metastatic, cytokine-refractory & $\mathrm{D}(\mathrm{II})$ & $\mathrm{D}(\mathrm{II})$ & GNR (III) & GNR (III) \\
\hline Pancreatic cancer & Advanced & $\mathrm{D}(\mathrm{III})$ & GNR (III) & GNR (III) & GNR (III) \\
\hline Colorectal cancer & Metastatic & $\mathrm{D}(\mathrm{III})$ & GNR (III) & GNR (III) & GNR (III) \\
\hline Multiple sclerosis & & $\mathrm{D}(\mathrm{III})$ & GNR (III) & & $\mathrm{CO}(\mathrm{II})$ \\
\hline Systemic scleroderma & & $\mathrm{D}(\mathrm{III})$ & GNR (III) & & $\mathrm{CO}(\mathrm{II})$ \\
\hline $\begin{array}{l}\text { Systemic lupus } \\
\text { erythematosus }\end{array}$ & & $\mathrm{D}(\mathrm{III})$ & GNR (III) & & $\mathrm{CO}(\mathrm{II})$ \\
\hline Crohn's disease & & GNR (III) & GNR (III) & & $\mathrm{CO}(\mathrm{II})$ \\
\hline Rheumatoid arthritis & & GNR (III) & GNR (III) & & $\mathrm{CO}(\mathrm{II})$ \\
\hline Vasculitis & & GNR (III) & GNR (III) & & $\mathrm{CO}(\mathrm{II})$ \\
\hline $\begin{array}{l}\text { Polymyositis- } \\
\text { dermatomyositis }\end{array}$ & & GNR (III) & GNR (III) & & $\mathrm{CO}(\mathrm{II})$ \\
\hline
\end{tabular}

${ }^{*} \mathrm{~S}$, standard of care (generally indicated in suitable patients); $\mathrm{CO}$, clinical option (can be carried after careful assessment of risks and benefits); D, developmental (further trials are needed); GNR, generally not recommended. Levels of evidence are displayed in braquets.

Abbreviations: CP, chronic phase; CR1/2/3, first/second/third complete remission; DIPSS, Dynamic International Prognostic Score System; IPI, International prognostic index; M3, promyelocytic leukaemia; RA, refractory anaemia; RAEB, refractory anaemia with excess blasts; RCMD, refractory cytopenia with multilineage dysplasia; SAML, secondary AML; TKI, tyrosine kinase inhibitors.

Adapted with permission from Sureda A, Bader $P$, Cesaro S, Dreger $P$, et al., Indications for allo- and auto-SCT for haematological diseases, solid tumours and immune disorders: current practice in Europe, 2015. Bone Marrow Transplant 2015, 50(8):1037-56; Copyright Nature Publishing Group. 


\subsection{Brief historical review}

The rational basis for bone marrow transplantation (BMT) stems from pioneer experiments performed in the post-World War II period in an effort to overcome the lethal complications of high-dose radiation exposure. ${ }^{(1,21)}$ Jacobson et al. demonstrated in mice that survival from otherwise lethal radiation exposure could be achieved with the shielding of the spleen and/or femur by a lead foil, ${ }^{(22,23)}$ and proposed the existence of "a substance of noncellular nature within the spleen and bone marrow" which protected the subjects from irradiation toxicity (humoral hypothesis). Further work by Lorenz et al. showing that infusion of spleen or bone marrow cells had a similar protective effect in irradiated rodents, ${ }^{(24)}$ introduced an alternative hypothesis which viewed the spleen and bone marrow cellular constituents as being responsible for the clinical recovery observed (cellular hypothesis). A few years later, several research groups provided definite evidence supporting the latter, documenting in mice that after irradiation and infusion of bone marrow or spleen cells the haematopoietic recovery was dependent on the cells derived from the donor grafts. ${ }^{(25-29)}$

Many of the factors known to be responsible for a successful bone marrow engraftment were established in early studies in murine BMT models: ${ }^{(30)}$ (1) Van Bekkum et al. showed that repopulation of the marrow spaces was effectively achieved if bone marrow cells were given intravenously; ${ }^{(31)}$ (2) conditioning with cyclophosphamide was sufficient for 
allogeneic engraftment; ${ }^{(32)}$ (3) successfully engrafted allogeneic bone marrow cells could induce an aggressive reaction against the host causing a wasting syndrome (secondary disease), now recognised as being graft-versus-host disease (GVHD); ${ }^{(33)}$ (4) genetic factors controlled the severity of the immune reaction of donor cells against the host; ${ }^{(34)}$ (5) GVHD could be prevented or ameliorated through immunosuppression with methotrexate. ${ }^{(35)}$

However, transferring the knowledge obtained from experimental animal models to humans was met with many challenges. The results from the initial attempts at translating the procedure into the clinic were disappointing: although in some of the patients treated with BMT aimed at reconstituting the BM following radiotherapy, successful engraftment and leukaemia remission were achieved, none survived for more than a few months, ${ }^{(36)}$ succumbing to early complications associated with bone marrow aplasia (e.g. bleeding, severe infections) or after developing severe fatal GVHD. These discouraging results tempered the clinicians' enthusiasm, with some prominent haematologists stating that "... these failures have occurred mainly because the clinical applications were undertaken too soon, most before even the minimum basic knowledge required to bridge the gap between mouse and patient had been obtained". (30)

Further research was subsequently conducted in dogs as it was predicted that the wide genetic diversity of out-bred dogs would be a much more suitable animal model for preclinical studies. ${ }^{(37)}$ Although the existence of murine histocompatibility systems had already been documented in the mid-1950s, ${ }^{(38)}$ it was only with Epstein et al. in 1968 that it was demonstrated 
that leukocyte antigen compatibility between donor and recipient was absolutely required for the success of allogeneic transplantation. ${ }^{(39)}$ Moreover, disparities involving minor histocompatibility antigens (miHA) were found to be equally capable of eliciting GVHD. ${ }^{(40)}$ On the other hand, mutual graft-versus-host tolerance was documented to be established after 3 to 6 months of methotrexate treatment, suggesting that suspension of immunosuppressive therapy would be possible thereafter. ${ }^{(41)}$

The encouraging results in pre-clinical models, together with the discovery of the Human Leukocyte Antigen (HLA) system by J. Dausset ${ }^{(42)}$ and J.J. Van Rood ${ }^{(43)}$ which permitted selection of HLA identical donors, inspired further trials of allogeneic HSCT between matched human siblings. Additionally, improvements in supportive care (in particular blood component transfusions, antibiotic/ antifungal / antiviral therapy, vascular access, and parenteral nutrition) lead to a reduction in pancytopenia associated complications and permitted the development of new high-dose conditioning regimens. ${ }^{(44)}$

In the late 1960s, Gatti et al., Bach et al. and deKoning et al. reported the first 3 successful cases of allogeneic marrow transplantation in patients with severe combined immunological deficiency using HLA-matched sibling donors, ${ }^{(45-47)}$ with a long term survival of over 25 years. ${ }^{(48)}$ In the following years, the Seattle Marrow Transplant Team described their results with leukaemia and aplastic anaemia patients transplanted after failure of conventional therapy, showing some long-term disease-free survival, in spite of still having a high transplant-related mortality. ${ }^{(49,50)}$ Due to the low success rate and morbidity 
associated with the procedure, at this stage, BMT was restricted to patients with immunoglobulin deficiencies, severe aplastic anaemia, or refractory advanced leukaemia/lymphoma who had HLA-matched sibling donors. This remained the leading practice for over a decade, until 1977 when Thomas et al. showed that patients in poorer conditions at transplantation had a significantly lower survival, ${ }^{(50)}$ suggesting that higher success rates would be achieved if transplantation occurred earlier in the course of the disease, when tumour burdens are reduced and patients are still in good medical condition.

Consistent with earlier findings in dogs, half of the patients receiving a transplant from an HLA-matched sibling donor and treated with methotrexate alone still developed GVHD. However, by associating cyclosporine A (CSA) or tacrolimus, GVHD management was significantly improved, ${ }^{(51-53)}$ to such an extent that these combinations of immunosuppressants are widely used to this day. Long-term survival of allogeneic transplanted patients introduced two new concepts into clinical practice: chronic GVHD and the graft-versus-leukaemia (GVL) effect. As it emerged that tumour was controlled both by the conditioning's cytotoxicity and by the effects of the donor immune system against malignant host cells, a link between GVL and GVHD was recognised. ${ }^{(54-}$ 56)

Gradually, allogeneic BMT began to be viewed as a valid treatment, not to be used as a desperate measure for patients with refractory end-stage disease, but rather as a planned procedure offered to patients in first complete remission of an acute leukaemia, in first blast phase of chronic myeloid leukaemia, or for as soon as a donor was identified in patients with aplastic 
anaemia. Concurrently, autologous HSCT was demonstrated to be an effective treatment option for patients with chemo- or radiotherapy- sensitive malignancies, such as lymphoid malignancies ${ }^{(57)}$ and some childhood tumours (e.g. neuroblastoma), ${ }^{(58)}$ as extramedullary toxicity of the conditioning became the sole limiting factor of dose intensification.

This new chapter in HSCT was marked by important breakthroughs (Figure 2):

- introduction of new conditioning regimens - the combination of high dose busulphan (BU) and cyclophosphamide $(\mathrm{CY})^{(59)}$ avoided irradiation, making HSCT possible in centres without access to total body irradiation (TBI);

- creation of a network of national donor registries ${ }^{(60)}$ - inclusion of tissue-typed volunteers in the donor pool extended the applicability of HCST to patients who didn't have HLA-matched sibling donors;

- recognition of the negative effect of blood transfusions on subsequent bone marrow grafts ${ }^{(61)}$ - transplant recipients can become sensitized to non-HLA antigens by receiving unirradiated transfusional support, increasing the risk of graft rejection;

- advances in immunogenetics of $\mathrm{HLA}^{(62)}$ - replacement of the serological typing system by molecular techniques of allele characterisation revealed the full heterogeneity of the major histocompatibility complex (MHC), and made possible a more adequate donor-recipient matching, improving the results of unrelated grafts to the level of HLA-identical sibling transplantation, at least for young patients; ${ }^{(63)}$ 


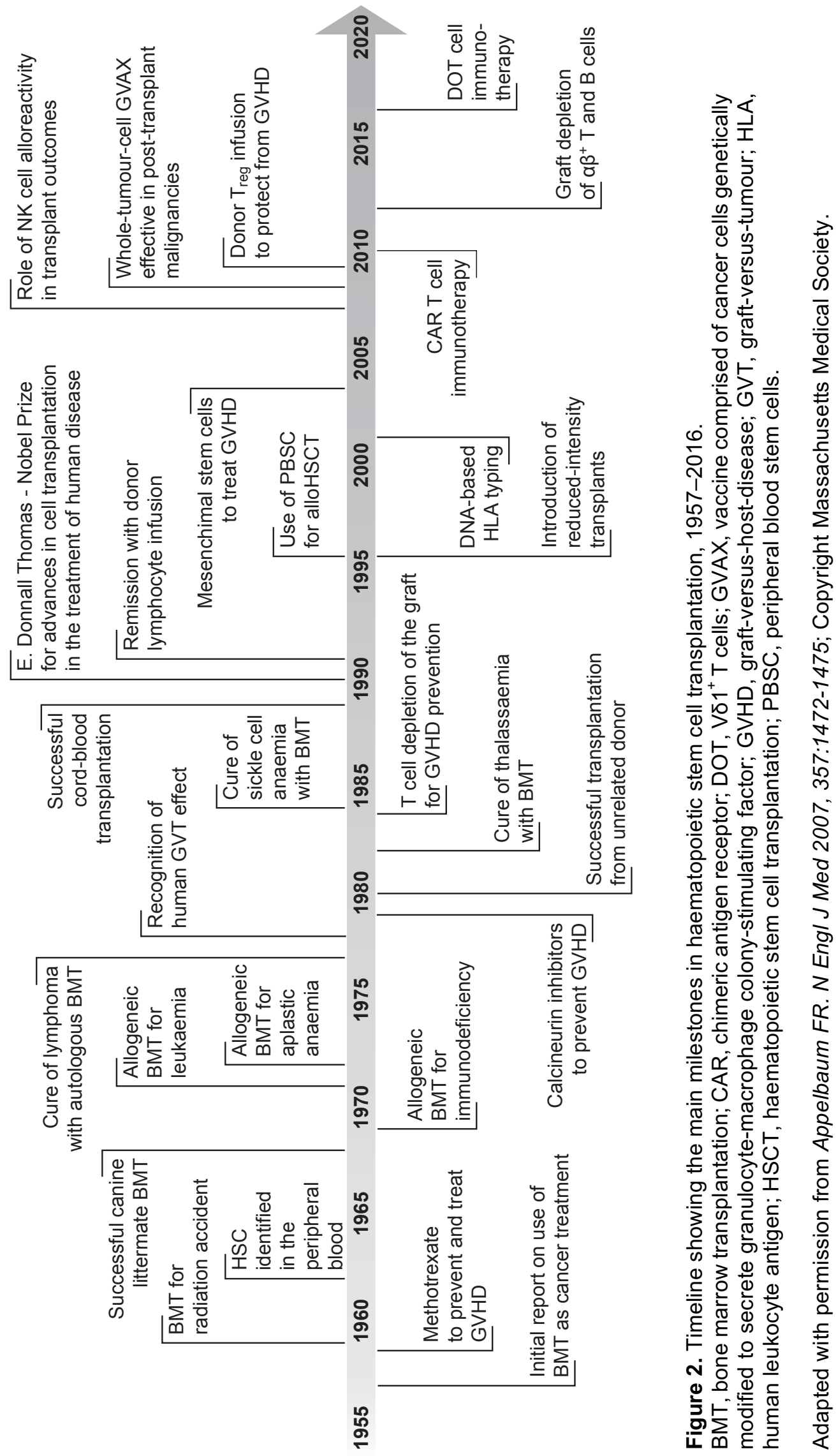


- use of alternative graft sources - the identification of the glycoprotein CD34 as a marker of haematopoietic progenitor cells and the development of centrifuge technology for bulk blood leukocyte collection provided the means to isolate stem cells from the peripheral blood ${ }^{(64)}$ and from umbilical cord blood. ${ }^{(13)}$

As the understanding of GVT biology grew, it became clear that the key to a successful alloHSCT resided in the immunologic elimination of malignant stem cells. This concept shifted the focus from the traditional myeloablative preparative regimens and led to the development of reduced-intensity conditioning (RIC) regimens extending HSCT to older and frailer patients. ${ }^{(65,66)}$ However, although safe and effective in patients with slow-growing cancers, the low transplant related mortality rate associated with $\mathrm{RIC}$ regimens may be offset by higher relapse rates in patients with advanced diseases. ${ }^{(67,68)}$

In recent years, research has focused on developing new strategies for increasing disease free survival. However, achieving a separation between GVHD and GVL has proved challenging. It has been shown that graft $\mathrm{T}$ cell depletion is associated with a lower incidence of both acute and chronic GVHD, but at the cost of a delayed immune reconstitution with an increased risk of infectious complications, and higher disease relapse rates. ${ }^{(69,70)}$ Moreover, adoptive immunotherapy through subsequent donor lymphocyte infusion (DLI), although effective in boosting immune reconstitution and treating leukaemia relapses, still carry the risk of triggering GVHD. ${ }^{(71)}$ Other approaches include: manipulation of the graft to reduce its alloreactive T cell component, either by eliminating activated $\mathrm{T}$ cells, ${ }^{(72)}$ selecting for memory $\mathrm{T}$ cells, ${ }^{(73,74)}$ inducing 
donor $\mathrm{T}$ cells tolerance for host-type alloantigens, ${ }^{(75)}$ or selecting for regulatory $\mathrm{T}$ cells; ${ }^{(76)}$ vaccinations against tumor-associated antigens; ${ }^{(77,78)}$ immunotoxin-based therapy; ${ }^{(79)}$ and targeted adoptive cellular immunotherapy. ${ }^{(80)}$

Altogether, HSCT is at present the treatment which provides the best chance for cure for many pathologies, including non-haematological malignancies and autoimmune diseases. 


\section{Graft-versus-host disease}

Despite significant progress in the past 20 years, GVHD is still a major barrier to successful HSCT, affecting $30-50 \%$ of transplanted patients, and remaining one of the leading causes of long-term morbidity and mortality (Figure 3). ${ }^{(81)}$ The risk of GVHD is multifactorial, increasing with certain conditioning regimens, graft type (peripheral blood stem cells $>$ bone marrow $>$ cord blood), type of HSCT (HLA-mismatched > HLA-matched unrelated > HLAmatched sibling), age of the donor, multiparous female donor, and age of the recipient.

This immunological complication of alloHSCT is caused by donor T cells recognizing the genetically disparate transplant recipient (host) as foreign, thus initiating an immune reaction that leads to tissue damage, which can be further amplified by disturbances in immunological reconstitution and failure to acquire immunological tolerance. ${ }^{(82)}$ 


\section{Causes of death}

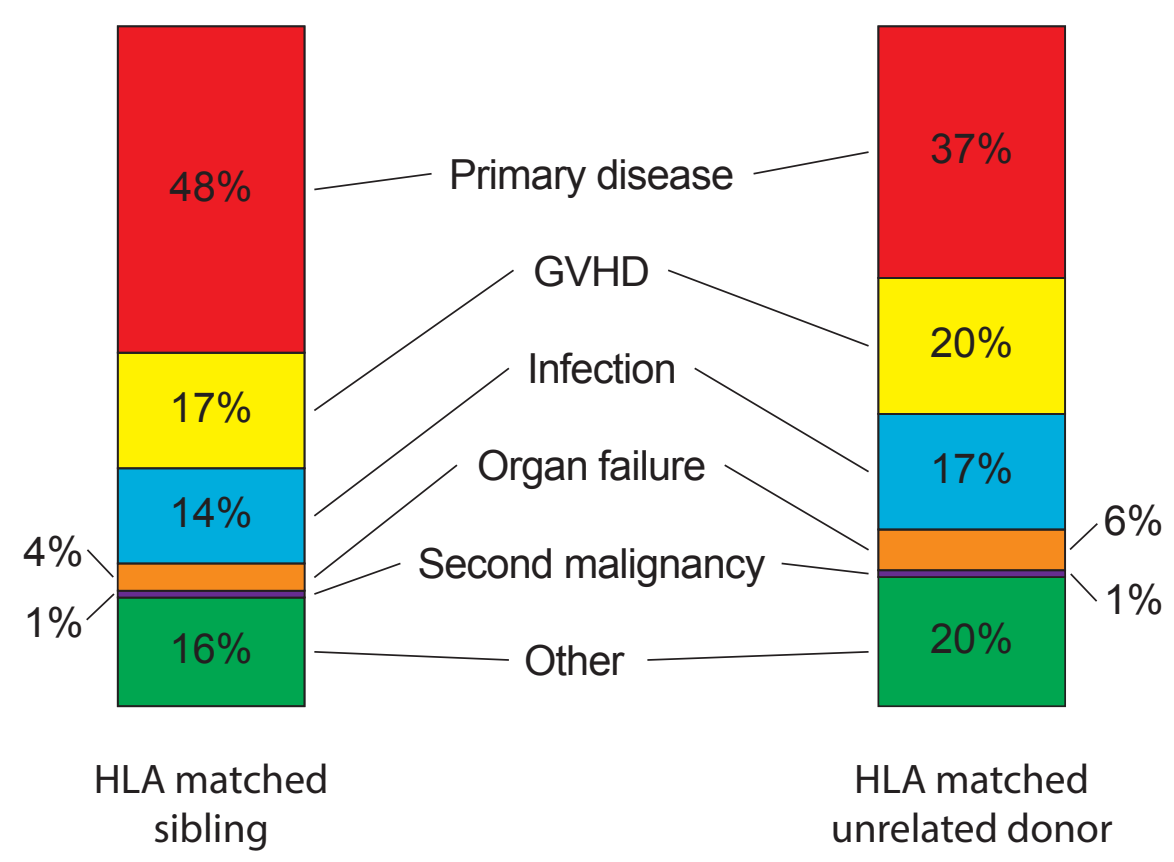

Figure 3. Causes of death after HLA-matched sibling (A) and unrelated donor (B) HSCT.

Adapted with permission from Pasquini MC, Zhu X. Current uses and outcomes of haematopoietic stem cell transplantation: CIBMTR Summary Slides, 2015; Copyright The Medical College of Wisconsin, Inc. and the National Marrow Donor Program. 


\subsection{Classification and clinical features}

Two main forms of GVHD can be recognised, acute and chronic. Although the classical distinction of the two forms of the disease, based on the time of its occurrence (before versus after D+100 post-HSCT) and the main clinical features, are still valid today, the boundaries between acute and chronic GVHD have become less rigid as there is cumulative evidence supporting that GVHD should be viewed as a continuum process (Table 3$){ }^{\left({ }^{(83)}\right.}$ Nevertheless the clinical manifestation of the disease remain central in the current classification of GVHD syndromes. While acute GVHD (aGVHD) affects primarily the skin (erythroderma), liver (cholestasis) and gut (diarrhoea with or without haematochezia) (Table 4), ${ }^{(84)}$ chronic GVHD (cGVHD) can present itself with a myriad of manifestations across multiple organs, most frequently, the skin (dermatosclerosis), mouth (xerostomia), eyes (xerophthalmia), vagina (scarring and stonis), esophagus (strictures), lung (bronchiolitis obliterans), and musculoskeletal system (fasciitis and joint stiffness) (Table 5). ${ }^{(83)}$

However, the pathophysiology underlying each of the syndromes is quite distinct. Whilst, aGVHD can be viewed as a distortion of the normal inflammatory cellular responses, involving alloreactive donor $\mathrm{T}$ cell-mediated cytotoxic damage to the recipient's tissues via cell-surface and secreted factors, ${ }^{(85)}$ cGVHD is mainly characterised by end-organ fibrosis, and its features resemble those of well-recognized autoimmune diseases, suggesting similar pathophysiology. ${ }^{(86)}$ 
Table 3. Distinguishing acute and chronic GVHD.

\begin{tabular}{lccc}
\hline Category & $\begin{array}{c}\text { Time of } \\
\text { symptoms }\end{array}$ & $\begin{array}{c}\text { aGVHD } \\
\text { features }\end{array}$ & $\begin{array}{c}\text { cGVHD } \\
\text { features }\end{array}$ \\
\hline \hline $\begin{array}{l}\text { Acute GVHD } \\
\text { Classic acute }\end{array}$ & & & \\
$\quad \begin{array}{l}\text { Persistent, recurrent or late onset acute } \\
\text { Chronic GVHD }\end{array}$ & $>100$ days & Yes & No \\
$\quad \begin{array}{ll}\text { Classic chronic } \\
\text { Overlap syndrome }\end{array}$ & Yes & No \\
\hline
\end{tabular}

aGVHD, acute GVHD; cGHVD, chronic GVHD

Adapted with permission from Apperley J, Masszi T. Chapter 13 - graft-versus-host disease. In: Apperley J, Carreras E, Gluckman E, Masszi T, editors. ESH-EBMT Handbook on Haematopoietic Stem Cell Transplantation. European School of Haematology, Paris; 2012. p. 216-33; Copyright European School of Haematology \& European Society for Blood and Marrow Transplantation.

Table 4. Organ involvement in acute GVHD.

\begin{tabular}{cccc}
\hline & Skin & Liver (bilirubin) & Gut (stool output/day) \\
\hline \hline $\begin{array}{c}\text { Stage } \\
0\end{array}$ & No GVHD rash & $<2 \mathrm{mg} / \mathrm{dl}$ & $\begin{array}{c}<500 \mathrm{ml} / \mathrm{day} \\
\text { or persistent nausea. }\end{array}$ \\
1 & $\begin{array}{c}\text { Maculopapular rash } \\
<25 \% \text { BSA }\end{array}$ & $2-3 \mathrm{mg} / \mathrm{dl}$ & $500-999 \mathrm{ml} / \mathrm{day}$ \\
2 & $\begin{array}{c}\text { Maculopapular rash } \\
25-50 \% \text { BSA }\end{array}$ & $3.1-6 \mathrm{mg} / \mathrm{dl}$ & $1000-1500 \mathrm{ml} / \mathrm{day}$ \\
3 & $\begin{array}{c}\text { Maculopapular rash } \\
>50 \% \text { BSA }\end{array}$ & $6.1-15 \mathrm{mg} / \mathrm{dl}$ & $\begin{array}{c}\text { Adult: }>1500 \mathrm{ml} / \mathrm{day} \\
\text { Severe abdominal pain } \\
\text { with or without ileus }\end{array}$ \\
\hline
\end{tabular}

BSA, body surface area

Adapted with permission from Jacobsohn DA. Acute graft-versus-host disease in children. Bone Marrow Transplant 2008, 41(2): 215-21; Copyright Macmillan Publishers Ltd. 


\subsection{Acute GVHD immunopathology}

Most of our current knowledge about the biology of GVHD is derived from observations made in animal models of the disease. Early studies established that the development of GVHD required: (1) an immunologically competent graft containing mature T cells; (2) inability of the recipient to reject the transplanted cells; and (3) expression of tissue antigens by the recipient absent in the transplant donor. ${ }^{(87-89)}$ The current conceptual model is that its pathogenesis can be divided into 5 sequential steps (Figure 4). ${ }^{(81,90,91)}$

\section{a) Priming of the immune response}

The first phase of acute GVHD is triggered by tissue injury induced by the primary disease, prior chemo- and radiotherapy treatments or infectious complications, and further exacerbated by the HSCT conditioning regimen. ${ }^{(90)}$ The damaged tissues produce a storm of pro-inflammatory cytokines (e.g. TNF- $\alpha$, IL-1, IL-6) $)^{(92-94)}$ which lead to the up-regulation of a variety of "danger signals", such as adhesion and costimulatory molecules, MHC antigens, and chemokines, activating host antigen presenting cells (APC). ${ }^{(95)}$

Damage of gastrointestinal mucosa and disruption of the intestinal microbiome are of particular importance in this process. ${ }^{(94)}$ Gastrointestinal toxicity is known to be one of the most important dose-limiting factors in highdose conditioning for HSCT. Changes to the permeability of the gut mucosal barrier allows bacterial and/or endotoxin translocation into the systemic 
A

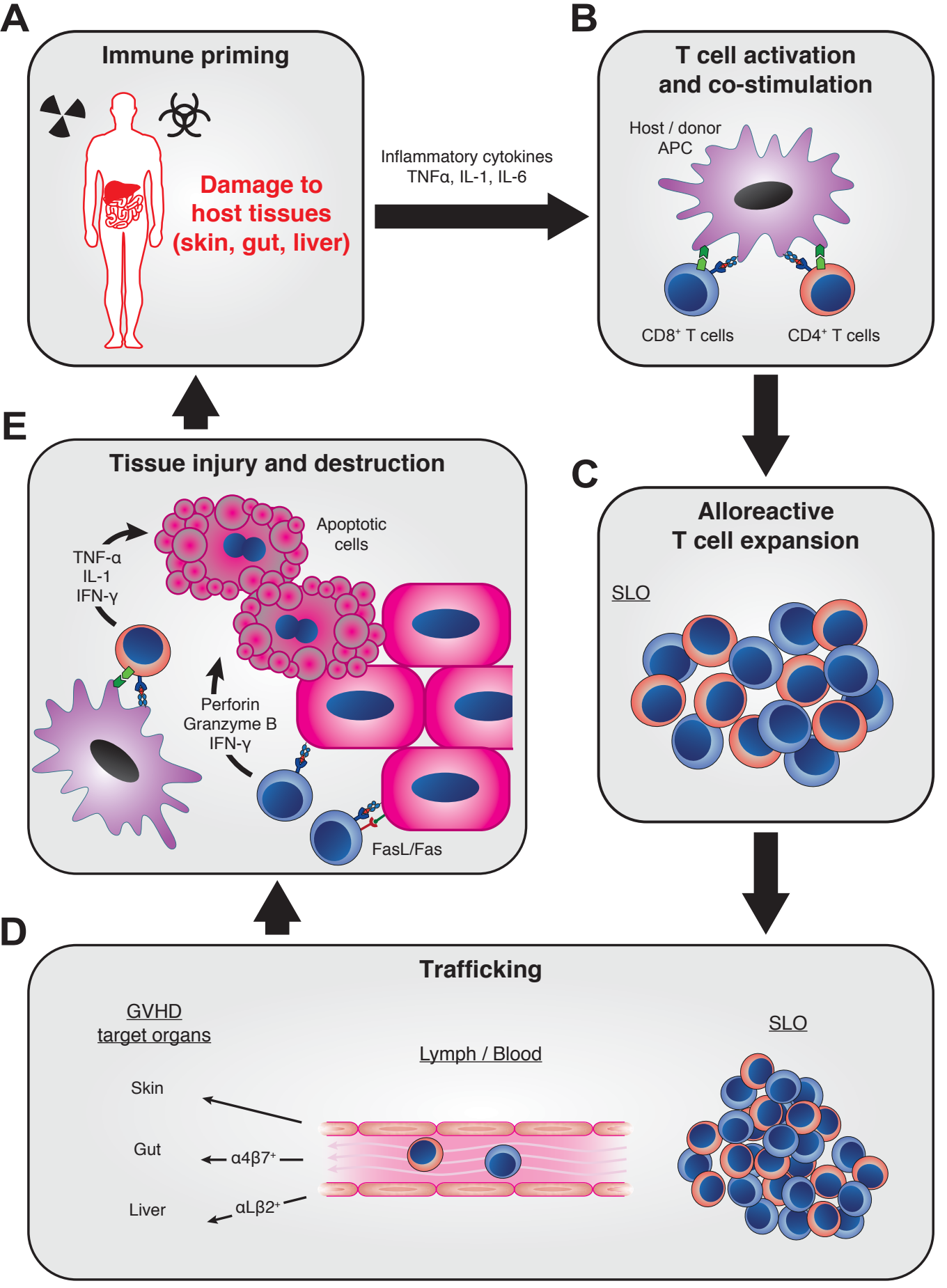

Figure 4. Progression of events in aGvHD development: immune priming (A), activation (B), T-cell expansion (C), T-cell trafficking (D) and host tissue injury (E). 
circulation. ${ }^{(96)}$ Endotoxins are potent stimulators of toll-like receptors, leading to the release of an array of cytokines that enhance tissue inflammation. ${ }^{(97)}$ Conversely, in murine HSCT models in which the integrity of intestinal barrier is protected, the severity of GVHD is significantly diminished. ${ }^{(98,99)}$

Recent studies looking into the biology of intestinal epithelial cells and the intestinal microbiota have unveiled a mechanistic link between changes in microbiome diversity, intestinal epithelial cell preservation and GVHD immunopathology. ${ }^{(100-103)}$ For instance, it has been shown that a decrease in the amount of the microbial metabolite butyrate, a short-chain fatty acid histone deacetylase inhibitor, in the intestinal tissue of alloHSCT recipients resulted in decreased acetylation of histone $\mathrm{H} 4$ in intestinal epithelial cells, leading to apoptosis. Moreover, direct intragastric administration of exogenous butyrate restored acetylation of histone $\mathrm{H} 4$, protected epithelial barrier by altering the expression ratio of anti-apoptotic to pro-apoptotic molecules and increasing the expression of proteins relevant for junctional integrity, which resulted in decreased GVHD severity. ${ }^{(100)}$

The role of TLR signalling has further been recognised in the skin; in studies where an activator of TLR7 was topically applied to the epidermis before inducing GVHD, extensive T cell infiltrates and GVHD histopathologic changes were only observed at the site of treatment. ${ }^{(104)}$ Other relevant molecules released following conditioning regimen-induced tissue damage, such as the damage-associated molecular patterns (DAMP), have been implicated in the induction of GVHD. For instance, binding of extracellular ATP to P2X7 receptor and activation of the inflammasome leads to the over-expression of 
co-stimulatory molecules on antigen presenting cells (APC). ${ }^{(105)}$

Altogether, these findings are consistent with the correlation observed between the risk of developing GVHD and the intensity of the HSCT conditioning regimens. ${ }^{(106-108)}$

\section{b) Activation of donor T cells}

Once primed, tissue APC migrate to the secondary lymphoid organs (SLO) where they induce $\mathrm{T}$ cell activation. This process requires cognate interaction between donor $\mathrm{T}$ cells and alloantigen presenting APC. The recognition of host allopeptide presented on the MHC of APC by donor lymphocyte $\mathrm{T}$ cell receptor (TCR) is not by itself sufficient to induce $\mathrm{T}$ cell activation; ${ }^{(109)}$ costimulatory signals, such as CD27, OX40, ICOS, CD40, 4-1BB, are required for $\mathrm{T}$ cell proliferation, differentiation and survival (Figure 5). ${ }^{(110,111)}$

It has been shown in murine models, that GVHD is primarily induced by donor $\mathrm{T}$ cell interactions with host-derived haematopoietic APC, ${ }^{(112)}$ even though, once initiated, both donor- and host-derived APC are sufficient to further promote $\mathrm{T}$ cell activation (Figure 6). ${ }^{(113)}$ Donor T cell activation can be achieved both by direct presentation on host APC (the peptide bound to allogeneic MHC molecules or allogeneic MHC molecules without peptide are recognised by donor T cells) and indirect presentation on donor APC (peptide generated by degradation of the allogeneic MHC molecules is presented on self-MHC). ${ }^{(114-116)}$ However, to this date, no study using stringent conditional ablation of individual APC subsets has been able to demonstrate the absolute requirement of any single recipient APC subset for GVHD induction. ${ }^{(95)}$ 


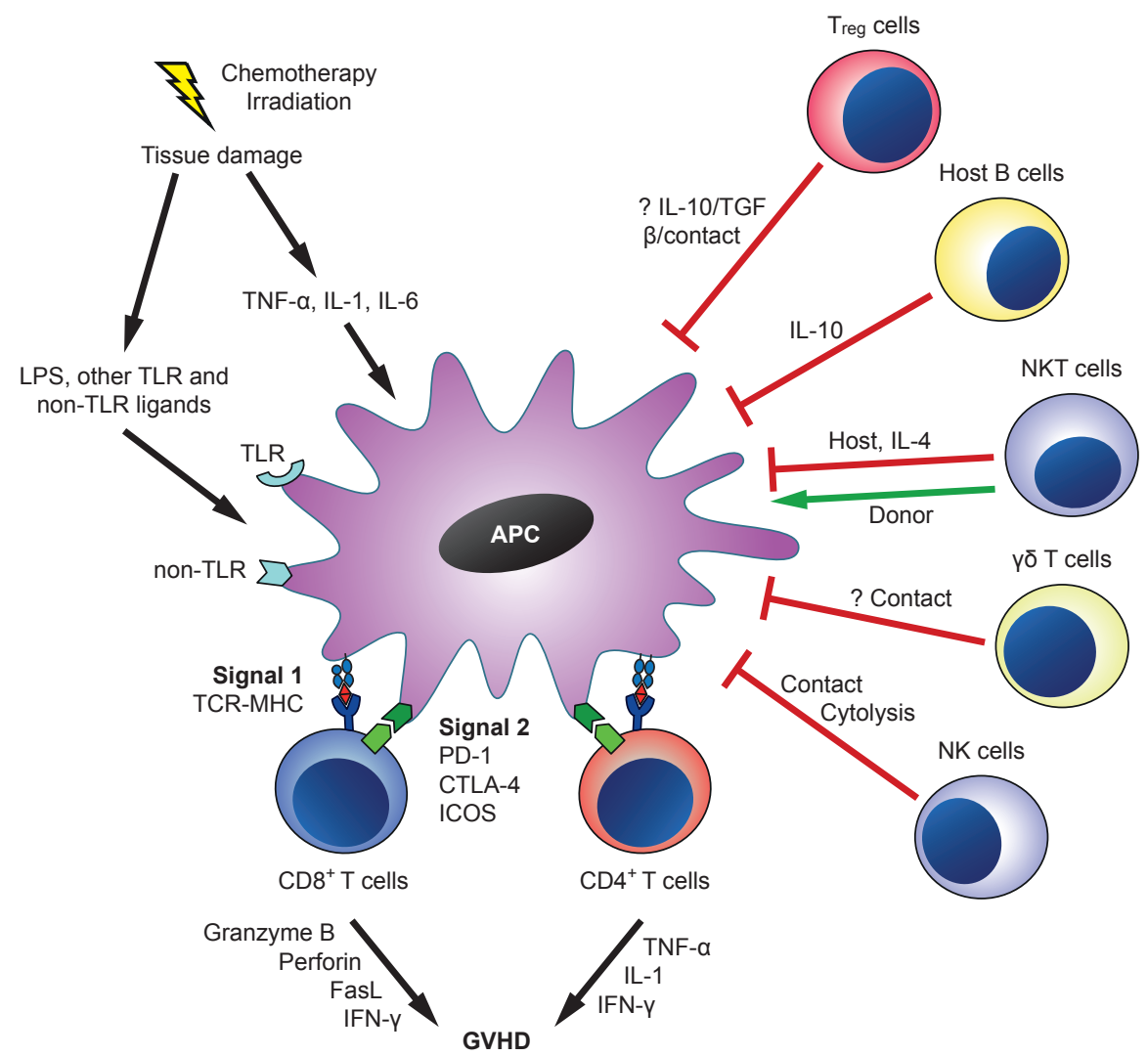

Figure 5. Critical interactions for induction of GVHD.

Adapted with permission from Paczesny S, Hanauer D, Sun Y, Reddy $P$. New perspectives on the biology of acute GVHD. Bone Marrow Transplant 2010, 45(1): 1-11; Copyright Nature Publishing Group. 
A

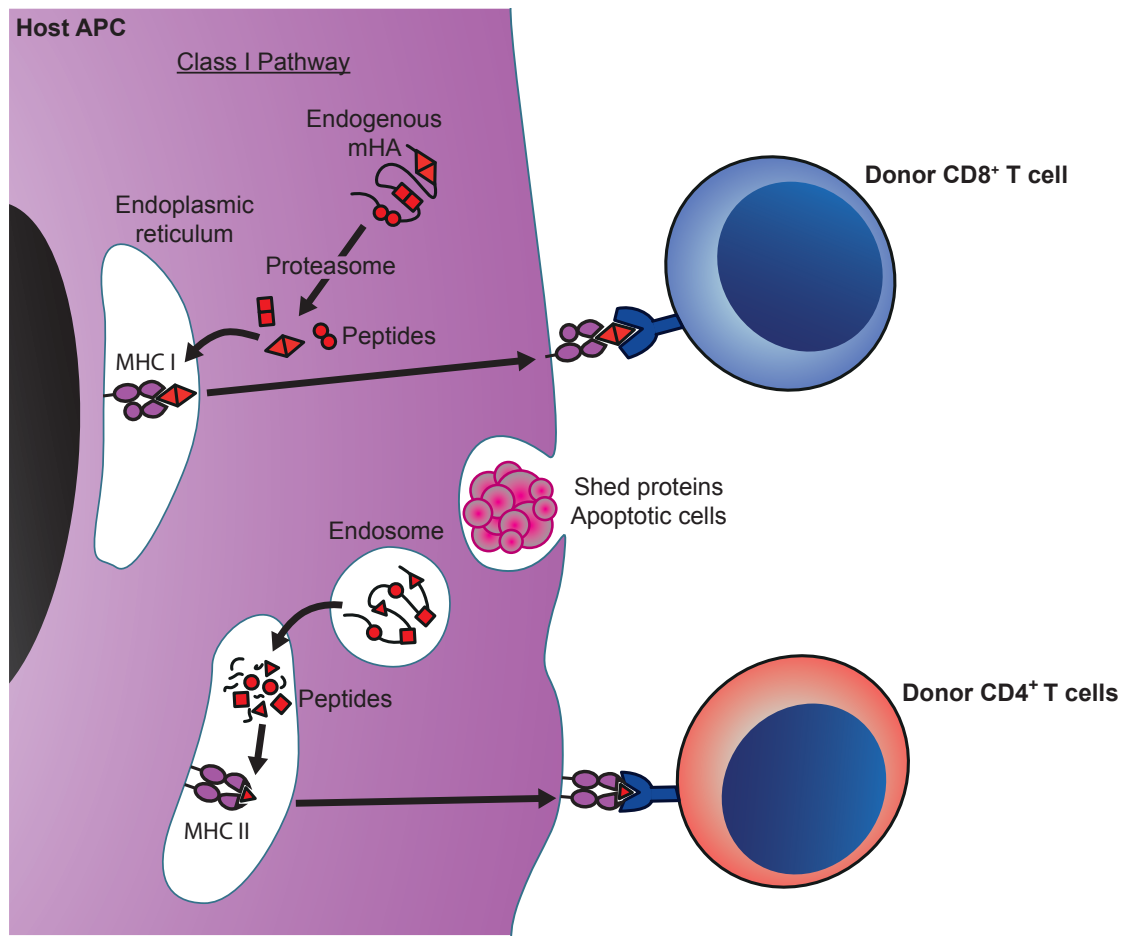

B

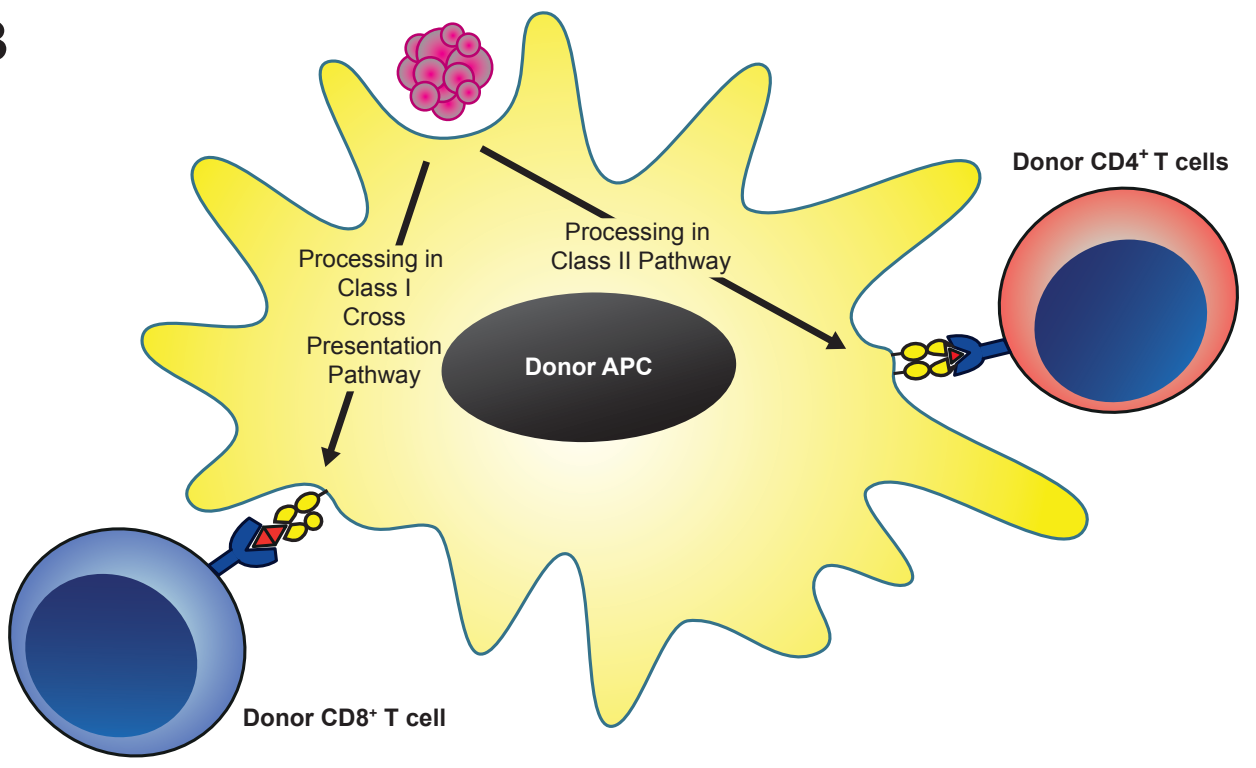

Figure 6. Antigen presentation in GVHD in MHC-matched allogeneic stem-cell transplantation. (A) Allogeneic immune reaction is initiated by donor $\mathrm{CD}^{+} \mathrm{T}$ cells recognizing the peptide products of polymorphic genes (miHAs). In the class I antigen presentation pathway, host APC processes cytosolic proteins through the proteasome and the resulting peptides, including miHAs, are transported into the endoplasmic reticulum, where loading onto MHC class I molecules occurs. Peptide-MHC class I complexes exported to the cell surface are then made available for antigen recognition by donor $\mathrm{CD} 8+\mathrm{T}$ cells. Initial $\mathrm{CD} 4{ }^{+} \mathrm{T}$-cell activation, on the other hand, can be directed against endocytosed antigens by host- or donor-derived APC and processed by the class II pathway. (B) When donor-derived APC have fully replaced the hostderived population, activation of donor $\mathrm{CD}^{+} \mathrm{T}$ cells would solely rely on cross-presentation of exogenously acquired antigens, through the uptake of apoptotic recipient or shed proteins in a process involving phagosome retrotranslocation. TAP, transporter associated with antigen processing.

Adapted with permission from Shlomchik WD. Graft-versus-host disease. Nat Rev Immunol 2007, 7(5): 340-52; Copyright Nature Publishing Group. 
The role of non-haematopoietic APC in the induction of GVHD is still controversial. Although it has been shown that donor $\mathrm{CD}^{+} \mathrm{T}$ cells can be activated by non-haematopoietic APC in the gastrointestinal tract, ${ }^{(117,118)}$ haematopoietic APC are critical for CD8+ T cell priming, particularly in the context of MHC-compatible but miHA-mismatched HSCT. ${ }^{(112)}$

\section{c) Alloreactive $\mathrm{T}$ cell proliferation and differentiation}

Following priming, donor T cells proliferate and differentiate into effector cells, a process characterised by gain of cytolytic function and ability to secrete cytokines, such as IL-1, IL-3, IL-7, GM-CSF, TNF- $\alpha$ and IFN-ץ. Differentiation into Th1/Tc1, Th2/Tc2 and Th17/Tc17 is dependent on the cytokine milieu. For instance, it has been shown that while in the presence of IL-12, CD4 ${ }^{+} \mathrm{T}$ cells differentiate into IFN-ץ producing Th1 cells, IL-4 induces differentiation into Th2 cells producing IL-4, IL-5, and IL-13; as for Th17 cells, TGF- $\beta$ and IL-6 is required for their differentiation, and IL-23 and IL-21 are critical for their expansion and survival. ${ }^{(119)}$ Although it has been demonstrated that the balance between Th1/Tc1, Th2/Tc2 and Th17/Tc17 subsets impacts the manifestation of GVHD, the exact contribution of each of these elements is still under active investigation. ${ }^{(120)}$

The pro-inflammatory molecules IFN-y, IL-2 and TNF- $\alpha$ produced by Th1/Tc1 cells have been implicated in the pathogenesis of GVHD, ${ }^{(92,96)}$ being linked to an increased mortality and to the classical syndrome characterised by weight loss, diarrhoea and skin changes. ${ }^{(121)}$ However, the role of some cytokines is still controversial, as IFN-y was shown to both regulate immune suppression and support cellular cytotoxicity. ${ }^{(122)}$ 
Conversely, Th2 polarization of $\mathrm{T}$ cells has been reported to reduce aGVHD. ${ }^{(123,124)}$ IL-2 and IL-4 treatment of donor mice was shown to induce generation of IL-4 and IL-10 producing Th2 cells which induced protection from tissue injury associated with GVHD when transferred into sublethally irradiated mice or nonirradiated F1 recipients. ${ }^{(125,126)}$ Furthermore, in miHA mismatch BMT models of GVHD, transplantation of Th2 polarized cells resulted in a significant reduction in GVHD associated mortality. ${ }^{(127)}$

Th17/Tc17 cell subsets have been proposed to have a dual role after HSCT, in particular on the gastrointestinal tract. IL-17 pro-inflammatory action is well established in several autoimmune diseases, ${ }^{(128-130)}$ and its role mediating GVHD has also been demonstrated in experimental models. ${ }^{(131)}$ On the other hand, like group 3 innate lymphoid cells (ILC3), ${ }^{(132,133)}$ Tc17 cells produce IL-22, a cytokine that acts on the intestinal epithelial stem cell compartment to limit apoptosis and preserve mucosal barrier function. ${ }^{(134)}$ However, with the onset of acute GVHD the differentiation, homeostasis and function of host ILC3 are compromised, and decrease in intestinal ILC3 is directly associated with enhanced intestinal tissue damage owing to a loss of IL-22. Post-HSCT Th17/Tc17 differentiation may thus be central for mucosal immunity reconstitution and restoration of its barrier function, but when dysregulated the primary pro-inflammatory effects are responsible for the induction of severe gastrointestinal damage.

\section{d) Migration of effector cells to GVHD target tissues}

After being retained in the SLO for a period of 2 to 3 days during which proliferation and differentiation occurs, ${ }^{(135)}$ effector $T\left(T_{\text {eff }}\right)$ cells migrate into the 
tissues in a process that involves changes in vascular permeability and requires specific selectin-ligand, chemokine-receptor, and integrin-ligand interactions. ${ }^{(136)}$

T cell egress from the lymphoid organs is thought to be mediated by sphingosine 1-phosphate (S1P) signalling. ${ }^{(137)}$ Experimental work using FTY720, an antagonist drug that targets S1P receptors, demonstrated that the expression of S1P receptor 1 on $\mathrm{T}$ cells and the establishment of a S1P gradient, low in the LN parenchyma and high at the exit site, was critical for $T$ cells to exit the SLO. Furthermore, in a murine model of GVHD, FTY720 treatment starting at the time of transplantation was shown to be an effective strategy to prevent target organ T cell infiltration. ${ }^{(138)}$

During differentiation, $\mathrm{T}$ cells up-regulate a number of chemokine receptors, such as CCR2, CCR5, CXCR3, and CXCR6, and produce IFN-Y and TNF- $\alpha$. These pro-inflammatory cytokines enter the circulation and induce host cells in the peripheral tissues to produce an array of chemokines (e.g. CXCL9, CXCL10, and CXCL11), ${ }^{(139)}$ creating an inflammatory environment that directs $\mathrm{T}_{\text {eff }}$ cells to the target organs. ${ }^{(136)}$

Upon entering the peripheral tissues, donor $\mathrm{T}$ cells induce a second wave of chemokine ligand production, including CCL3, CCL4, and CCL5, which help maintain target organ infiltration when CXCL9, CXCL10, and CXCL11 production has subsided. ${ }^{(140,141)}$

In addition to chemokines and their receptors, migration of inflammatory cells to target organs is regulated by the expression of selectins, integrins and their ligands. ${ }^{(142,143)}$ Homing of donor $\mathrm{T}$ cells to intestinal mucosa requires 
interaction between the $\alpha 4 \beta 7$ integrin (also known as lymphocyte Peyer patch adhesion molecule - LPAM), expressed by T cells, and its ligand, the mucosal vascular addressin cell adhesion molecule 1 (MadCAM-1), ${ }^{(143,144)}$ while entry of effector $\mathrm{T}$ cells into the lung and liver involves interactions between $\alpha \mathrm{L} \beta 2$ (lymphocyte function-associated antigen 1 - LFA-1)/ intercellular adhesion molecule 1 (ICAM-1), and aLß2/ICAM-1, -2 , and -3 , respectively. ${ }^{(136)}$

\section{e) Target tissue destruction by $T_{\text {eff }}$ cells}

GVHD target organ damage is the result of a complex immune response in which cytotoxic T cells (CTL), NK cells and inflammatory cytokines induce antigen-dependent and -independent cell death (Figure 4-E).

Data from murine models of GVHD using T cell donors deficient for FasL, perforin or granzyme $\mathrm{B}$, or by the in vivo administration of neutralizing anti-FasL antibodies, demonstrated that perforin and granzyme B dependent cytolysis and Fas-mediated apoptosis are two of the main mechanisms of cytotoxicity employed by CTL. ${ }^{(145,146)}$ Supporting these observations, it has been reported in human patients a positive correlation between the serum levels of soluble FasL and Fas and the clinical severity of GVHD. ${ }^{(147,148)}$ Notably, different cytotoxic pathways dominate in each organ, while CTL use preferentially the Fas/FasL pathways to induce liver damage, the perforin and granzyme pathways appear to be more important in the gastrointestinal tract and skin. ${ }^{(91,149)}$

Although CTL and NK cells differ in the receptors involved in the recognition of target cells, the mechanism by which they induce apoptosis are fundamentally the same. However, NK cell cytotoxicity appears to be mediated 
primarily through perforin/granzyme-dependent processes, even though they express FasL and are likely to use these molecules to kill certain target cells. ${ }^{(150)}$

Other death ligand receptor signalling pathways that have been suggested to be implicated in GVHD target tissue damage are TWEAK/Fn14, ${ }^{(151)}$ TRAIL, ${ }^{(152)}$ LIGHT/LT $\beta .{ }^{(153)}$ However, their distinct role in GVHD is still uncertain. ${ }^{(154,155)}$

The secretion of inflammatory mediators, e.g. TNF- $\alpha$, IL-1, IFN- $y$, and nitric oxide (NO), can also induce GVHD target organ injury independent of any cognate interaction. The relevance of TNF- $\alpha$ in GVHD immunopathology has been made clear in trials where prophylaxis and treatment of steroid-resistant GVHD patients with a TNF- $\alpha$ blocker have shown efficacy, particularly against gastrointestinal disease. ${ }^{(156-158)}$

IL-1 has long been considered the second major pro-inflammatory cytokine in the effector phase of acute GVHD, ${ }^{(159)}$ being predominantly secreted in the spleen and skin. ${ }^{(160)}$ Several studies in murine models supported this concept, as neutralization of IL-1 significantly reduced GVHD mortality ${ }^{(161)}$ and IL-1 blockade using IL-1Ra downregulated Th17 cell differentiation and reduced the severity of GVHD. ${ }^{(162)}$ However, its relevance in human GVHD pathophysiology is uncertain as IL-1Ra treatment in a controlled randomised clinical trial has proved to be insufficient to reduce GVHD or to improve survival. ${ }^{(163)}$

IFN-y has been reported to have ambivalent effects in vivo, as it can both amplify and suppress acute GVHD. ${ }^{(122,164)}$ Regarding amplification, IFN-Y 
increases the expression of several surface molecules such as chemokine receptors, MHC proteins, and adhesion molecules in APC and stromal cells, enhancing their response to pro-inflammatory stimuli. ${ }^{(165)}$ Moreover, NO mediated damage to the intestinal epithelium and the epidermis is primarily dependent on donor-derived IFN- $y \cdot{ }^{(166)}$ However, by accelerating the apoptosis of activated donor T cells, IFN-y can both dampen GVHD severity and induce profound deficits in immune function after HSCT. ${ }^{(167)}$

Further to the traditional Th1/Th2 paradigm of $\mathrm{T}$ cell polarisation, increasing evidence supports the role of Th17/Tc17 cells in the pathophysiology of GVHD. Studies in which the cytokines that drive Th17 differentiation were neutralised, in particular IL-6 and IL-23, showed a significant reduction in the severity of acute GVHD. ${ }^{(168-171)}$ In accordance with these results, blockage of transcription factors critical to Th17 generation (e.g. RORyt, RORa, IRF-4) resulted in diminished GHVD, and was associated with lower levels of IL-17A and TNF- $\alpha$ being detected in the serum and GVHD target organs. ${ }^{(172)}$

Many of the cytokines produces by Th17 cells (IL-21, IL-22, TNF-a, GM-CSF, IL-17F) have now been linked to the development of both acute and chronic GVHD. ${ }^{(173)}$ However, their individual pathogenic role is still difficult to interpret independently of coexpressed Th1 cytokines. Additionally, it is now recognised that Th17 cells display a broad context-dependent plasticity, associated with higher in vivo survival and self-renewal capacity, and are able of acquiring functional features of Th1 cells, a characteristic that may prove critical for GVHD pathology. ${ }^{(174)}$ 


\section{Pre-clinical models of GVHD}

As described above, some of the major advances in HSCT and much of the current understanding of GVHD biology was derived from experimental work largely in preclinical animal models with rodents, dogs and, to lesser extent, non-human primates (Table 6). ${ }^{(91)}$ As an outbred large animal model, with a diversity of leukocyte antigens similar to the HLA, the dog has been invaluable in the development of conditioning regimens, ${ }^{(175,176)}$ prophylaxis and treatment of GVHD, ${ }^{(41,51,177)}$ and understanding of the pathology of GVHD. ${ }^{(178)}$ However, such models are greatly limited by cost and experimental group size, time/duration of experiments, lack of detailed or validated immune reagents, and inability to assess GVT effects due to the unavailability of compatible tumour lines. ${ }^{(179)}$

Since the early experimental work performed in the mid- $20^{\text {th }}$ century, the inbred mouse model has had a central place in the progression of our knowledge in HSCT. Having a highly controlled genetic background, with a variety of genetic modified strains available, and being kept on a consistent diet, under tightly regulated specific pathogen-free housing conditions, they provide the ideal experimental system in which researchers can dissect individual variables of disease and exclude the effect of external factors that interfere disease development and progression. ${ }^{(179)}$ Even though the aforementioned characteristics of the murine models warrant great reproducibility of the results, 
some disparities in radio-sensitivity and GVHD severity ${ }^{(180)}$ have been observed that may arise from differences in animals from different vendors, related to differences in feed, microbiomes, specific pathogen-free environments, or genetic drift. $^{(181-183)}$

Furthermore, there are significant inter-specie differences that need to be considered before broad conclusions can be drawn and translated into clinical practice:(91) distinct conditioning regimen modalities induce different severities of tissue inflammation; ${ }^{(104,184)}$ the depth of genetic disparities between donor and recipient affect the kinetics and pathophysiology of GVHD and GVT;(185) differences in ratio and homing characteristics of the immune cells infused affect the pattern of GVHD or GVT induction; ${ }^{(186)}$ the recipient's microbiome and its changes due antibiotic prophylaxis and/or treatment can affect the response to the conditioning regimen, marrow engraftment and GVHD development; ${ }^{(100,187,188)}$ post-HSCT immune reconstitution and GVHD severity are influenced both by the donor an recipient's age, related to increased immune senescence, impaired tissue repair mechanisms, increased alloreactivity of recipient APC and lymphocytes, and underlying chronic low-grade inflammation status. ${ }^{(189,190)}$ a 
Table 6. Advantages and disadvantages of various preclinical model systems.

\begin{tabular}{|c|c|c|}
\hline Species & Advantages & Disadvantages \\
\hline $\begin{array}{l}\text { Murine } \\
\text { model }\end{array}$ & $\begin{array}{l}\text { - MHC well defined } \\
\text { (major mismatch vs minor } \\
\text { mismatch HSCT models) } \\
\text { - Variety of genetic knock out } \\
\text { models available } \\
\text { - Environmental/housing control } \\
\text { - Relatively short life span } \\
\text { - Predictable, reproducible } \\
\text { response } \\
\text { - Allows for specific experimental } \\
\text { intervention testing }\end{array}$ & $\begin{array}{l}\text { - Transplantation models only } \\
\text { recapitulate single organ-specific } \\
\text { GVHD pathologies } \\
\text { - SPF environment, cannot mimic the } \\
\text { interactions between the host } \\
\text { immune system and environmental } \\
\text { pathogens } \\
\text { - Microbiome diversities do not mimic } \\
\text { the complexity of the human scenario } \\
\text { - Tumours are derived from cell lines } \\
\text { rather than spontaneous tumours }\end{array}$ \\
\hline $\begin{array}{l}\text { Cani } \\
\text { mod }\end{array}$ & $\begin{array}{l}\text { - Similar environmental } \\
\text { - Masposures to humans } \\
\text { leukocyte antigens similar to } \\
\text { human leukocyte antigens } \\
\text { - Mimics both unrelated and } \\
\text { related haploidentical bone } \\
\text { marrow transplant in the clinic } \\
\text { - Spontaneous haematological } \\
\text { tumour development }\end{array}$ & $\begin{array}{l}\text { - Haplotyping of dog leukocyte } \\
\text { antigens is very difficult } \\
\text { - Genetic knockout models are not } \\
\text { available } \\
\text { - Requires specialized veterinary } \\
\text { technician assistance } \\
\text { - Housing requirements limit the } \\
\text { numbers of animals per cage, } \\
\text { increasing housing costs and space } \\
\text { needed } \\
\text { - Difficult to standardize treatments, as } \\
\text { may require different conditioning } \\
\text { based on differences in breed, age, } \\
\text { body fat, parasite status etc. }\end{array}$ \\
\hline $\begin{array}{c}\text { Non-human } \\
\text { primate } \\
\text { model }\end{array}$ & $\begin{array}{l}\text { - MHC locus closely resembles } \\
\text { that of humans and may mimic } \\
\text { the different degrees of MHC } \\
\text { mismatch observed in the } \\
\text { transplantation setting } \\
\text { - Share similar near-universal } \\
\text { exposure to pathogens } \\
\text { - Similar response to conditioning } \\
\text { regimens, as well as toxicity and } \\
\text { GVHD incidence; cellular } \\
\text { immunity composition and } \\
\text { markers are similar to humans }\end{array}$ & $\begin{array}{l}\text { - Haplotyping of non-human primates } \\
\text { is difficult } \\
\text { - Lack of spontaneous tumours } \\
\text { - Lack of reagents available for } \\
\text { immunological studies } \\
\text { - Limited numbers of non-human } \\
\text { primates available for studies, and } \\
\text { prohibitive costs } \\
\text { - Variability in the amount of irradiation } \\
\text { conditioning required due to } \\
\text { difference in body fat, age or health } \\
\text { status makes standardization difficult } \\
\text { - Requires specialized veterinary } \\
\text { technician assistance, and housing } \\
\text { requirements limit the numbers of } \\
\text { animals per cage, increasing the } \\
\text { housing costs and space needed } \\
\text { - Ethical concerns regarding primates } \\
\text { cognitive and emotional abilities, and } \\
\text { very long lifespan }\end{array}$ \\
\hline
\end{tabular}

SPF, specific pathogen-free

Adapted with permission from Stolfi JL, Pai CC, Murphy WJ. Preclinical modeling of haematopoietic stem cell transplantation - advantages and limitations. FEBS J 2016, 283(9): 1595-606; Copyright John Wiley and Sons. 


\subsection{Murine models}

Current murine models of HSCT can be broadly grouped into those in which GVHD is directed to MHC (class I, class II, or both) or to single or multiple miHA only, and they usually involve the transplantation of bone marrow (as a source of haematopoietic stem cells), supplemented with donor lymphocytes, into lethally irradiated allogeneic recipients. ${ }^{(180)}$ Depending on the biological question being addressed, adjustments to the number, phenotype and time point of transfer of donor T cells can be made.

Several strain combinations of donor-recipient pairs have been studied and the dominant features of each model characterised. ${ }^{(191)}$ For instance, based on the differences in MHC class I and/or II molecules (Figure 7 and Table 7) or minor histocompatibility antigens it can be predicted whether the effectors of GVHD are either $\mathrm{CD}^{+}$or $\mathrm{CD}^{+} \mathrm{T}$ cells, or both (Table 8). GVHD tissue injury in the context of a full MHC mismatch, both for class I and II molecules (e.g. B6 $\rightarrow$ BALB/c) is primarily dependent on $\mathrm{CD}^{+}{ }^{\mathrm{T}}$ cells, with $\mathrm{CD}^{+}$ T cells providing only additive pathology. ${ }^{(154)}$ These models of CD4 ${ }^{+}$-dependent GVHD are characterised by the development an inflammatory "cytokine storm”, capable of inducing damage in the target tissues without requirement for cognate $\mathrm{T}$ cell interaction with $\mathrm{MHC}$ in situ. ${ }^{(192)} \mathrm{By}$ contrast, $\mathrm{CD}^{+} \mathrm{T}$ cellmediated GVHD injury (e.g. $\mathrm{B} 6 \rightarrow \mathrm{bm} 1)$ requires the TCR to engage $\mathrm{MHC}$ on target tissue in order to trigger the cytolytic machinery. ${ }^{(193)}$ 


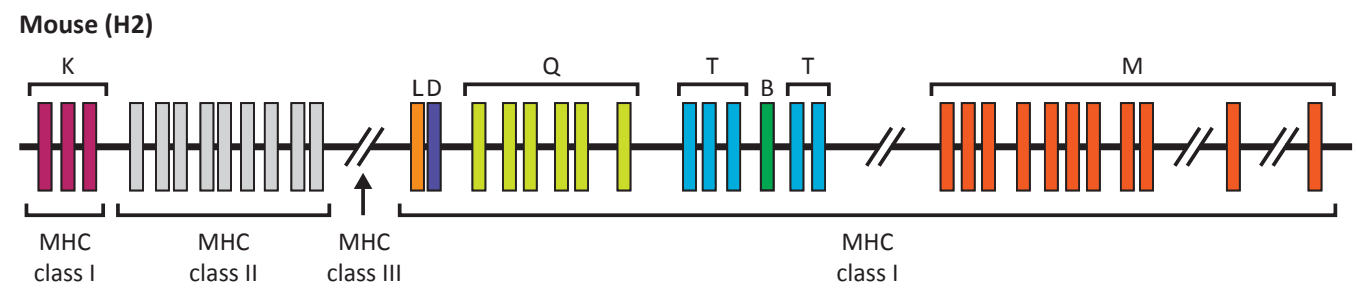

Figure 7. Genomic map of the mouse MHC complex

Table 7. H-2 haplotypes and alloantigens of some of the most commonly used laboratory mouse strains.

\begin{tabular}{|c|c|c|c|c|c|c|c|c|}
\hline \multirow{2}{*}{ Mouse Strains } & \multirow{2}{*}{$\begin{array}{c}\text { MHC } \\
\text { Haplotype }\end{array}$} & \multicolumn{3}{|c|}{ MHC Class I } & \multicolumn{2}{|c|}{ MHC Class II } & \multicolumn{2}{|c|}{ MHC Class Ib } \\
\hline & & $\mathrm{H}-2 \mathrm{~K}$ & $\mathrm{H}-2 \mathrm{D}$ & $\mathrm{H}-2 \mathrm{~L}$ & I-A & I-E & Qa-2 & Qa-1 \\
\hline $129 /-$ & $b$ & $b$ & $b$ & null & $b$ & null & a & $b$ \\
\hline$A / J$ & a & k & $d$ & $d$ & $\mathrm{k}$ & k & a & a \\
\hline BALB/B & $b$ & $b$ & $b$ & null & $b$ & null & a & $b$ \\
\hline $\mathrm{BALB} / \mathrm{C}$ & $d$ & $d$ & $d$ & $d$ & $d$ & $d$ & a & b \\
\hline $\mathrm{C} 3 \mathrm{H} / \mathrm{He}$ & $\mathrm{k}$ & $\mathrm{k}$ & $\mathrm{k}$ & null & $\mathrm{k}$ & $\mathrm{k}$ & $\mathrm{b}$ & b \\
\hline C57BL/6 & b & b & $b$ & null & $b$ & null & a & b \\
\hline C57BL/10 & b & b & b & null & b & null & a & b \\
\hline C57L/J & bc & $b$ & $b$ & null & $b$ & null & a & b \\
\hline $\mathrm{CBA} / \mathrm{J}$ & $\mathrm{k}$ & $\mathrm{k}$ & $\mathrm{k}$ & null & $\mathrm{k}$ & $\mathrm{k}$ & b & b \\
\hline DBA/1 & $q$ & $q$ & $q$ & $q$ & $q$ & null & a & - \\
\hline $\mathrm{DBA} / 2$ & $d$ & $d$ & $d$ & $d$ & $d$ & $d$ & a & $b$ \\
\hline FVB/N & $q$ & $q$ & $q$ & $q$ & $q$ & null & - & - \\
\hline LP/J & $\mathrm{bc}$ & $b$ & $b$ & null & $b$ & null & - & - \\
\hline NOD & g7 & $d$ & $b$ & null & g7 & null & a & - \\
\hline $\mathrm{P} / \mathrm{J}$ & $\mathrm{p}$ & $p$ & $p$ & $\mathrm{p}$ & $\mathrm{p}$ & $\mathrm{p}$ & $b$ & - \\
\hline $\mathrm{RF} / \mathrm{J}$ & $\mathrm{k}$ & k & k & null & k & $\mathrm{k}$ & $b$ & - \\
\hline SWR/J & q2 & $q$ & $q$ & $q$ & $q$ & null & a & a \\
\hline
\end{tabular}


Table 8. Commonly used mouse models of HSCT.

\begin{tabular}{|c|c|c|c|}
\hline Donor strain & Recipient strain & Genetics & $\mathrm{T}$ cell dependence \\
\hline \multicolumn{4}{|l|}{ MHC-mismatched } \\
\hline C57BL/6 $\left(\mathrm{H}-2 \mathrm{D}^{\mathrm{b}}\right)$ & BALB/C $\left(H-2 D^{d}\right)$ & $\begin{array}{l}\text { Mismatched for MHC-I, } \\
\text { MHC-II and miHA }\end{array}$ & $\mathrm{CD}^{+}$and $\mathrm{CD} 8^{+}$ \\
\hline $\mathrm{C} 3 \mathrm{H} / \mathrm{HeJ}\left(\mathrm{H}-2 \mathrm{D}^{\mathrm{k}}\right)$ & C57BL/6 $\left(H-2 D^{b}\right)$ & $\begin{array}{l}\text { Mismatched for MHC-I, } \\
\text { MHC-II and miHA }\end{array}$ & $\mathrm{CD}^{+}$and $\mathrm{CD} 8^{+}$ \\
\hline C57BL/6 $\left(\mathrm{H}-2 \mathrm{D}^{\mathrm{b}}\right)$ & $\mathrm{B} 6 \mathrm{C} 3 \mathrm{~F} 1\left(\mathrm{H}-2 \mathrm{D}^{\mathrm{k} / \mathrm{b}}\right)$ & $\begin{array}{l}\text { Mismatched for MHC-I, } \\
\text { MHC-II and miHA }\end{array}$ & $\mathrm{CD}^{+}$and $\mathrm{CD}^{+}$ \\
\hline C57BL/6 (H-2D $)$ & B6D2F1 $\left(H-2 D^{b / d}\right)$ & $\begin{array}{l}\text { Mismatched for MHC-I, } \\
\text { MHC-II and miHA }\end{array}$ & $\mathrm{CD}^{+}$and $\mathrm{CD} 8^{+}$ \\
\hline $\mathrm{C} 57 / \mathrm{BI} 6\left(\mathrm{H}-2 \mathrm{D}^{\mathrm{b}}\right)$ & $\mathrm{B} 6 \mathrm{AF} 1\left(\mathrm{H}-2 \mathrm{D}^{\mathrm{b} / \mathrm{a}}\right)$ & $\begin{array}{l}\text { Mismatched for MHC-I, } \\
\text { MHC-II and miHA }\end{array}$ & $\mathrm{CD}^{+}$and $\mathrm{CD} 8^{+}$ \\
\hline C57BL/6 $\left(H-2 D^{b}\right)$ & B10.BR $\left(H-2 D^{k}\right)$ & $\begin{array}{l}\text { Mismatched for MHC-I, } \\
\text { MHC-II and miHA }\end{array}$ & $\mathrm{CD}^{+}$and $\mathrm{CD} 8^{+}$ \\
\hline C57BL/6 $\left(\mathrm{H}-2 \mathrm{D}^{\mathrm{b}}\right)$ & $\begin{array}{l}\text { B6.C-H2bm1 (bm1) } \\
\text { (H2b background } \\
\text { with mutation at MHC I) }\end{array}$ & MHC I mismatch & $\mathrm{CD}^{+}$ \\
\hline C57BL/6 $\left(\mathrm{H}-2 \mathrm{D}^{\mathrm{b}}\right)$ & $\begin{array}{c}\text { B6.C-H2bm12 }(\mathrm{bm} 12) \\
\text { (H2b background } \\
\text { with mutation at MHC II) }\end{array}$ & MHC II mismatch & $\mathrm{CD}^{+}$ \\
\hline \multicolumn{4}{|l|}{ miHA mismatched } \\
\hline B10.BR $\left(H-2 D^{k}\right)$ & $\mathrm{CBA}\left(\mathrm{H}-2 \mathrm{D}^{\mathrm{k}}\right)$ & miHA mismatches & $\mathrm{CD}^{+}$ \\
\hline$B 10 . D 2\left(H-2 D^{d}\right)$ & $\mathrm{DBA} / 2\left(\mathrm{H}-2 \mathrm{D}^{\mathrm{d}}\right)$ & miHA mismatches & $\begin{array}{c}\mathrm{CD}^{+} \\
\text {(minor } \mathrm{CD} 8^{+} \text {contribution) }\end{array}$ \\
\hline B10.D2 $\left(H-2 D^{d}\right)$ & BALB/C $\left(H-2 D^{d}\right)$ & miHA mismatches & $\begin{array}{c}\mathrm{CD}^{+} \\
\text {(minor } \mathrm{CD}^{+} \text {contribution) }\end{array}$ \\
\hline C3H.SW (H-2D $\left.{ }^{b}\right)$ & C57BL/6 $\left(H-2 D^{b}\right)$ & miHA mismatches & $\begin{array}{c}\mathrm{CD}^{+} \\
\text {(with } \mathrm{CD}^{+} \text {help) }\end{array}$ \\
\hline C57BL/6 $\left(\mathrm{H}-2 \mathrm{D}^{\mathrm{b}}\right)$ & $129 / \mathrm{Sv}\left(H-2 D^{b}\right)$ & miHA mismatches & $\begin{array}{c}\mathrm{CD}^{+} \\
\text {(with } \mathrm{CD}^{+} \text {help) }\end{array}$ \\
\hline
\end{tabular}

Adapted with permission from Schroeder MA, DiPersio JF. Mouse models of graft-versus-host disease: Advances and limitations. Dis Model Mech 2011, 4(3): 318-33; Copyright Elsevier Inc. 
Due to their capacity to induce the full spectrum of clinically relevant GVHD, allowing the in vivo and ex vivo measurement of immunologic pathways, the MHC disparate systems have been the most commonly used model employed to dissect the immunologic mechanisms of GVHD. However, as MHC-mismatched transplants in humans are not commonly performed, miHA-mismatched models of acute GVHD are considered to be better representative of human biology. ${ }^{(191)}$

Similar to what is found in humans, miHA-mismatched models exhibit less morbidity than MHC-mismatched models, but still result in lethal acute GvHD. Interestingly, in mouse miHA-mismatched HSCT models, differences in MHC haplotypes (Figure 7) influence the type and specificity of the T-cell response underlying the GVHD phenotype. ${ }^{(191)}$ For example, in the B10.D2 $\rightarrow$ BALB/C or DBA/2 $\left(H-2 D^{d}\right)$ models, acute GVHD is primarily dependent on $\mathrm{CD}^{+} \mathrm{T}$ cells and less on $\mathrm{CD}^{+} \mathrm{T}$ cells, ${ }^{(194)}$ while in $\mathrm{C} 3 \mathrm{H} . \mathrm{SW} \rightarrow \mathrm{B} 6$ and $\mathrm{B} 6 \rightarrow 129 / \mathrm{Sv}\left(\mathrm{H}-2 \mathrm{D}^{\mathrm{b}}\right), \mathrm{GvHD}$ depends primarily on $\mathrm{CD}^{+} \mathrm{T}$ cells. ${ }^{(193)}$

Much of the described variability in these miHA-mismatched HSCT models is believed to be related to the fact that the disparities between donor and recipient are in multiple antigens. ${ }^{(195)}$ Although more than 200 miHA loci have been described in murine models using congenic mouse strains, immune responses are restricted to a few dominant miHA, including $\mathrm{H} 4, \mathrm{H} 7, \mathrm{H} 13, \mathrm{H} 28$, H60, and H-Y. ${ }^{(196-201)}$ Model-specific GVHD causing mechanisms and pattern of target organ involvement can be a result of the different tissue distribution of 
dominant $\mathrm{miHA}^{(202)}$ or differences in $\mathrm{MHC}$ affinity for antigens found in certain target tissues. ${ }^{(191)}$

To evaluate the relevance of individual miHA in triggering GVHD, several antigen-specific transgenic TCR models have been established. ${ }^{(191)}$ By restricting the specificity of the donor $\mathrm{T}$ cells to a single peptide epitope it is possible to overcome the effect of antigen-independent bystander activation of T cells and investigate several aspects of GVHD, including the role of cross presentation of antigens, antigen affinity, and antigen-induced T-cell activation and proliferation. ${ }^{(191)}$ Examples of TCR-transgenic mice used in GVHD research include the MataHari and Marilyn systems $(\mathrm{H}-\mathrm{Y}),{ }^{(118)}$ TEa system (H2-E $\left.{ }^{\mathrm{a}}\right),{ }^{(203)}$ D011.10 and OT-I (ovalbumin). ${ }^{(204,205)}$

Clinical data from MHC-matched HSCT show an increased risk of developing GVHD in male patients receiving grafts from female donors $(\mathrm{F} \rightarrow \mathrm{M}){ }^{(206)}$ in a process that appears to be dependent on $\mathrm{H}$-Y-specific alloresponses. ${ }^{(207-209)}$ Due to its ubiquitous tissue expression both in human and mice, research focused on $\mathrm{H}-\mathrm{Y}$ dependent alloreactions in GVHD have proven to be particularly useful, not only to study the pathologic mechanisms underlying gender mismatch HSCT, but also to dissect the biology of immune dysfunction in the context of miHA disparities in general. ${ }^{(118,210,211)}$

To further explore the cellular and molecular mechanism involved in the pathogenesis of GVHD, several genetically modified strains have been created. The usage of knockout or transgenic mice as donors or recipients in HSCT has allowed the study of the effect of specific cell populations or molecules on the development of alloreactive responses. For example: the usage of 
$\left(\beta 2 m^{-/-} \rightarrow B 6\right)$ chimeras as recipients for HSCT in miHA-mismatch model of GVHD, created a system in which haematopoietic cells are unable to present antigen through MHC-I, revealed that antigen presentation by haematopoieticderived APC is required but not obligatory for the induction of optimal $\mathrm{CD} 8^{+} \mathrm{T}$ cell mediated GVHD; ${ }^{(118)}$ transfer of $\mathrm{Ccr}^{-1-} \mathrm{T}$ cells in the context of HSCT corroborated the role of CCR5-CCL5 interactions both for the influx of donor T cells into the small intestine and for the induction of acute GVHD; ${ }^{(212)}$ by using perforin-deficient $\left(\mathrm{Prf1}^{-1-}\right)$ and granzyme B-deficient $\left(\mathrm{Gzmb}^{-/-}\right)$mice as T cells donors in MHC-mismatched model of GVHD it was demonstrated that perforin/granzyme-dependent mechanisms are required for MHC-I restricted acute GvHD but only have a minor role in MHC-II restricted acute GVHD. ${ }^{(213)}$

However, usage of systems in which genes are constitutively silenced or activated, or where cell populations are altogether absent, requires caution in the interpretation of the results as there are some caveats that need to be considered, in particular regarding developmental abnormalities induced by the genetic alteration. The current controversy regarding the role of Langerhans cells (LC) in the development of cutaneous acute GVHD is an example of such a situation. Being one of the few populations of professional APC that are resistant to the conditioning regimens and persist for long periods after transplantation, LC were regarded as having an important role in GVHD. Merad et al. supported this hypothesis demonstrating that administration of donor T cells to bone marrow-chimeric mice with persistent host Langerhans cells resulted in marked cutaneous GVHD, but were unable to induce skin damage in mice whose host Langerhans cells had been replaced by donorderived ones. ${ }^{(214)} \mathrm{Li}$ et al. contested these results by studying the development 
of cutaneous acute GVHD in Langerin-DTA recipients, a system which selectively lacks LC. In this model it was observed that the absence of recipient LC did not abrogate the development of GVHD. ${ }^{(215)}$ However, the authors failed to demonstrate that the constitutive LC depletion did not affect the skin's immune baseline, rendering other cell populations capable of inducing GVHD.

Alternative inducible models have now been created which allow for selective and time limited manipulations of the systems. Still in the context of the study of Langerhans cell biology, Dr Clare Bennett developed the Langerin-DTR model which allows for the selective depletion of LC upon treatment with diphtheria toxin (DT). ${ }^{(216)}$ Using Langerin-DTR mice as recipients in HSCT experiments, and treating them with DT only a few days before HSCT, a previous PhD student in Prof Chakraverty and Dr Clare Bennett's lab, Dr Thomas Conlan, showed that at the time of transplantation the only change to the recipients skin immune network was the absence of LC in the epidermis. In this highly controlled system, it was confirmed that host LC are required for the development of GVHD. ${ }^{(217)}$

Although animal models have many limitations that hinder the direct extrapolated of results into clinical application, preclinical modelling remains a valuable tool, allowing the simplification of complex systems and analysis of pathogenesis at the single-variable level in a controlled, reproducible manner.(179) 


\section{Unanswered questions in GVHD}

The results obtained in the animal models and in the clinical trials have been crucial for advancing HSCT practice over the last few years, leading to the development of new modalities of treatment and disease management. However, there are still some major gaps in our understanding of GVHD that require further research.

Although acute GVHD is a systemic disorder, the organs commonly targeted are consistently the gastrointestinal tract, the liver and the skin. It has been proposed that this pattern is due to the inherent pro-inflammatory milieu of these barrier organs, and that it may reflect differences in the distribution of antigens to which donor alloreactive T cells respond. ${ }^{(218)}$ However, it is well recognised that the conditioning regimen induces an inflammatory status that is not organ specific. ${ }^{(219)}$ Moreover, in experimental models using transgenic donor T cells that recognises a ubiquitously expressed antigen (e.g. MataHari), the pattern of organ involvement is maintained. ${ }^{(118)}$

The current scheme of GVHD development is based on data from models using myeloablative regimens and early transfer of donor T cells. ${ }^{(220)}$ The advent of reduced intensity conditioning regimens and delayed DLI, introduced the concept of late-onset acute GVHD. Arising at a point when 
conditioning-induced inflammation is subsiding, the pathophysiologic processes responsible for its onset have not yet been fully identified. ${ }^{(90)}$

The biology of chronic GVHD remains poorly defined, and although specific aspects of its pathophysiology are recapitulated by some animal models, none has been able to reproduce the most frequent modes of induction (i.e. acute GVHD evolving into chronic GVHD; chronic GVHD developing as immunosuppression is weaned). ${ }^{(221)}$

Moreover, the separation between the biologic pathways responsible for GVHD and GVL effect remain elusive; consequently, current approaches for GVHD prevention and treatment are broad-spectred and impact negatively on GVL responses and on immune reconstitution. ${ }^{(180)}$ As systemic corticosteroid therapy remains the standard primary therapy for GVHD, the development of steroid-refractory disease represents a life-threatening incident whose underlying mechanisms are still not understood. ${ }^{(90)}$

Recent advances in proteomics, gene profiling and whole genome sequencing have given new insights into the genetic basis of GVHD and to potential risk factors associated with the development of GVHD. ${ }^{(191)}$ However, thus far, these studies have failed to identify biomarkers with diagnostic significance which may change the paradigm of GVHD treatment from prophylactic to pre-emptive, and provide new means of improving the ability to predict the severity and kinetics of the disease. 


\section{Project rationale}

The current conceptual model of acute GVHD elegantly summarizes its biology, but in the attempt to deconvolute the complexity of the immune reactions involved in GVHD immunopathology to a linear, stepwise sequence of events compartmentalised into discrete stages, it fails to recognise the dynamic nature of the system, where cell populations play a diversity of roles depending on milieu, space and time. Indeed, most of the knowledge on the cellular and molecular mechanisms involved in GVHD pathogenesis come from a collection of results obtained in studies mainly focused on the spleen, lymph nodes (LN) and blood, at individual time points, from multiple model systems.

These SLO-centric approaches have thus failed to take into consideration the potential of interactions occurring in the peripheral tissues having a determinant role in regulating the effector phase of GVHD immunopathology. Data from experimental models of infection ${ }^{(222-225)}$ support the concept of a "two-hit" model of the immune response, in which the range the $\mathrm{T}$ cell effector function is not fixed at priming, but rather depend on additional antigen-dependent interactions with APC in the tissues. Such a model, if validated in the acute GVHD setting, could be useful for defining the determinants of target organ specificity. 
In the last years, new molecular tools such as gene expression profiling and genomic sequencing have provided the means to better characterise the changes that immune cells undergo during differentiation, and uncover important clues regarding the mechanisms underlying the establishment of protective responses against infections and the pathophysiology of several immune mediated diseases. Microarray and RNA-seq based technologies have also been used in GVHD studies to investigate differences between experimental groups. Most commonly, these studies employ classical tools which are based on the analysis of differential gene expression, and subsequent classification according to gene ontology annotation. Although this methodology has succeeded in identifying genes that are up- or downregulated in specific conditions (i.e. GVHD vs no GVHD; treated vs untreated; genetically modified vs wild-type), the pathophysiological significance of these findings is frequently difficult to determine, as the gene sets are often heterogeneous and biologically unrelated. Also, depending on the study design and on the heterogeneity of the populations included in each sample, the data may not be able to identify which cell type is involved. Moreover, this approach does not distinguish cause or effect nor how the gene expression changes occurs, and it fails to take into consideration gene-gene interactions which may be critical for normal cellular function or may be relevant for GVHD pathogenesis. $^{(226)}$

Advances in computational power have led to the development of more powerful bioinformatics tools which, instead of using preconceived thresholds of fold change and statistical significance to identify differentially expressed genes, analyse the correlation patterns among genes to generate networks of 
co-expressed and co-regulated genes. ${ }^{(227,228)}$ Weighted gene correlation network analysis (WGCNA) is one of such applications which can be used for finding clusters (modules) of highly correlated genes and for summarizing them using the module eigengene (defined as the first principal component of a given. module) or the intramodular hub genes (defined as the genes with the highest intramodular connectivity). ${ }^{(229)}$ By relating modules to one another and to external sample traits, new candidate biomarkers or therapeutic targets can be identified. ${ }^{(22)}$ These systems biology methods have been successfully applied in various biological contexts, identifying functionally related genes, enabling a more systematic and global interpretation of gene expression data. ${ }^{(230,231)}$

In this regard, the work in this thesis represents a novel approach to the study of GVHD, designed to evaluate the changes in the cellular programs of donor $\mathrm{CD}^{+} \mathrm{T}$ cells as acute GVHD develops in clinically relevant murine models of miHA-mismatch HSCT. By analysing the development of the alloreactive immune response at different sites and time points, within the same host, and employing both classical and systems biology methodologies to analyse the data obtained, this study constitutes an innovative multi-dimensional approach which provides valuable insights into the plasticity of $\mathrm{CD}^{+} \mathrm{T}_{\text {eff }}$ cell programs in the setting of acute GVHD. 


\section{Hypothesis}

GVHD target organs are major participants in shaping tissue injury, providing idiosyncratic signals that regulate alloreactive $\mathrm{CD}^{+} \mathrm{T}$ cell effector function.

\section{Aims and objectives}

- Develop a "systems immunology" methodology to characterise the transcriptional response of donor $\mathrm{CD}^{+} \mathrm{T}_{\text {eff }}$ cells as they traffic to multiple sites during the evolution of GVHD.

- Describe the main differences in donor $\mathrm{CD}^{+} \mathrm{T}_{\text {eff }}$ cell gene expression between the SLO and the GVHD target organs.

- Define tissue-specific alloreactive T cell gene signatures and identify the associated cellular programs.

- Validate the gene signatures against independent data sets and across different species.

- Identify the cellular and molecular mechanisms underlying the establishment of tissue-specific gene signatures. 
II. Materials \& Methods 


\section{Animal work}

\subsection{Mice}

C57BL/6 and 129/Sv mice were purchased from Charles River Laboratories (Margate, UK) and bred in house by UCL Biological Services (Royal Free Hospital, UK). C57BL/6 Langerin.DTREGFP (Langerin-DTR) mice were kindly provided by Bernard Malissen and Adrien Kissenpfennig (Université de la Mediterrannée, France) and bred in house. C57BL/6 TCR transgenic anti-HY (MataHari) mice were kindly provided by Jian Chai (Imperial College London, UK) and bred in house. Ifngr $1^{-/-}$mice were acquired from The Jackson Laboratory (USA) and bred in house.

Animals used as recipients for HSCT were $10-20$ weeks old, and those used as donors were 8 - 16 weeks old.

All procedures were conducted in accordance with the United Kingdom Home Office Animals (Scientific Procedure) Act of 1986, and were approved by the Ethics and Welfare Committee of the Comparative Biology Unit, Royal Free and University College London Medical School, London.

\subsection{Haematopoietic stem cell transplantation}

HSCT was performed as described before with minor modifications. ${ }^{(210)}$ Briefly, recipient mice were lethally irradiated (11 Gy TBI, split into two fractions 
over a period of 48 hours, at D-2 and D0) and reconstituted 4 hours later with $5 \times 10^{6}$ bone marrow cells, $2 \times 10^{6} \mathrm{CD}^{+}$splenocytes and $1 \times 10^{6} \mathrm{CD}^{+}$ splenocytes, administered by intravenous injection through the tail vein. Isolation of $\mathrm{CD}^{+}$and $\mathrm{CD}^{+} \mathrm{T}$ cells was performed by immunomagnetic selection of $\mathrm{CD}^{+}$or $\mathrm{CD}^{+}$splenocytes using Manual MACS ${ }^{\circledR}$ Cell Separation Technology (QuadroMACS Separator, LS columns, CD4 (L3T4) MicroBeads, CD8a (Ly-2) MicroBeads; Miltenyi, Germany), according to the manufacturer's instructions.

In the B6 $\rightarrow 129$ model: $129 / \mathrm{Sv}$ male mice $\left(\mathrm{CD} 45.2^{+} /\right.$Thy $\left.-1.2^{+}\right)$were used as recipients and $\mathrm{C} 57 \mathrm{BL} / 6$ female mice $\left(\mathrm{CD} 45.1^{+} /\right.$Thy $\left.-1.1^{+}\right)$were used as bone marrow and splenocyte donors (alloBMT group); or C57BL/6 female mice $\left(\mathrm{CD} 45.2^{+} /\right.$Thy $\left.-1.2^{+}\right)$were used as recipients and C57BL/6 female mice $\left(\mathrm{CD} 45.1^{+} /\right.$Thy $\left.-1.1^{+}\right)$were used as bone marrow and splenocyte donors (synBMT group) (Figure 8).

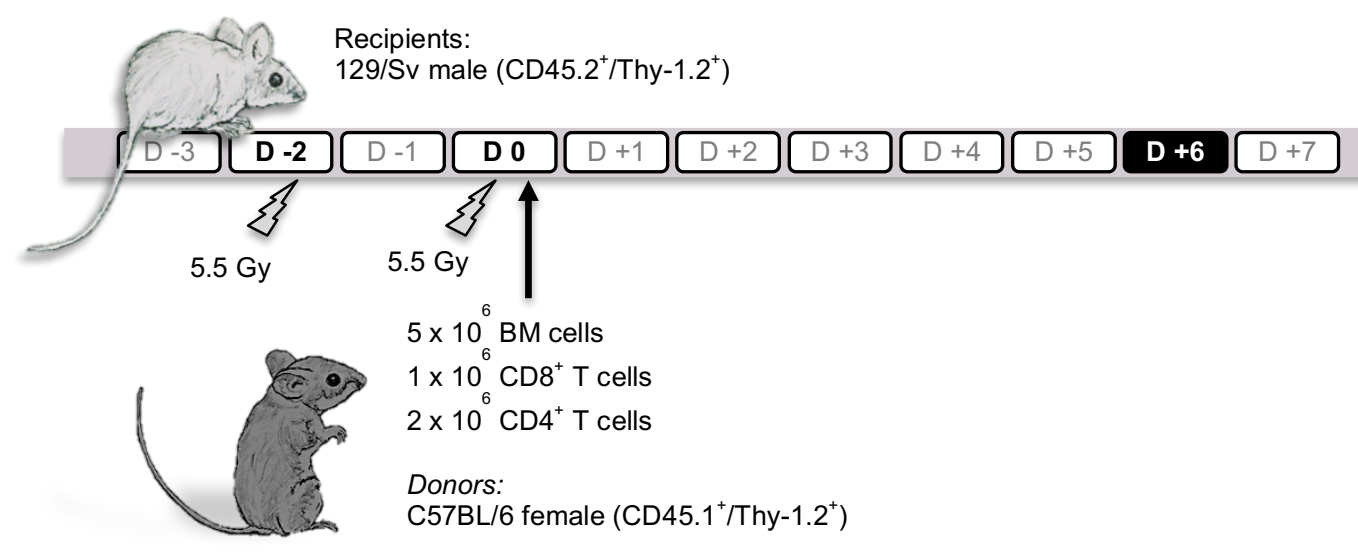

Figure 8. B6 $\rightarrow 129$ model.

In the MataHari T cell model: C57BL/6 male mice (CD45.2 $\left.2^{+} / T_{h y} 1.2^{+}\right)$ were used as recipients, C57BL/6 female mice $\left(\mathrm{CD} 45.2^{+} /\right.$Thy $\left.1.2^{+}\right)$were used 
as bone marrow and $\mathrm{CD}^{+}$splenocyte donors, and MataHari female mice $\left(\mathrm{CD} 45.2^{+} /\right.$Thy $\left.1.1^{+}\right)$were used as $\mathrm{CD}^{+}$splenocyte donors (alloBMT); C57BL/6 female mice $\left(\mathrm{CD} 45.2^{+} /\right.$Thy $\left.1.2^{+}\right)$were used as recipients, C57BL/6 female mice $\left(\mathrm{CD} 45.2^{+} /\right.$Thy $\left.1.2^{+}\right)$were used as bone marrow and $\mathrm{CD} 4^{+}$splenocyte donors, and MataHari female mice $\left(\mathrm{CD} 45.2^{+} /\right.$Thy $\left.1.1^{+}\right)$were used as $\mathrm{CD} 8^{+}$splenocyte donors (synBMT) (Figure 9).

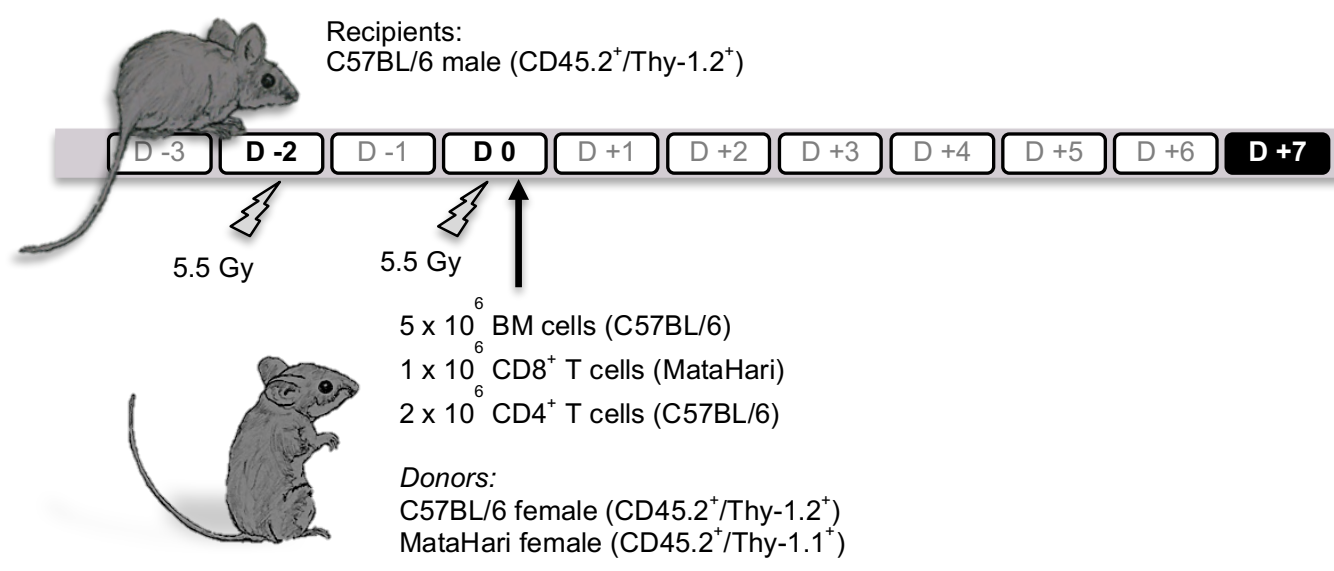

Figure 9. MataHari T cell model.

In the adapted MataHari T cell $\rightarrow$ Langerin-DTR model: Langerin-DTR male mice $\left(\mathrm{CD} 45.2^{+} /\right.$Thy $\left.1.2^{+}\right)$were used as recipients, C57BL/6 female mice $\left(\mathrm{CD} 45.2^{+} /\right.$Thy $\left.1.2^{+}\right)$were used as bone marrow and $\mathrm{CD} 4^{+}$splenocyte donors, and MataHari female mice (CD45.2 $2^{+}$Thy $\left.1.1^{+}\right)$were used as $C D 8^{+}$splenocyte donors (alloBMT); Langerin-DTR female mice (CD45.2 ${ }^{+} /$Thy $\left.1.2^{+}\right)$were used as recipients, C57BL/6 female mice $\left(\mathrm{CD} 45.2^{+} /\right.$Thy $\left.1.2^{+}\right)$were used as bone marrow and $\mathrm{CD}^{+}$splenocyte donors, and MataHari female mice (CD45.2 ${ }^{+} /$Thy $1.1^{+}$) were used as CD8 ${ }^{+}$splenocyte donors (synBMT) (Figure 10). 


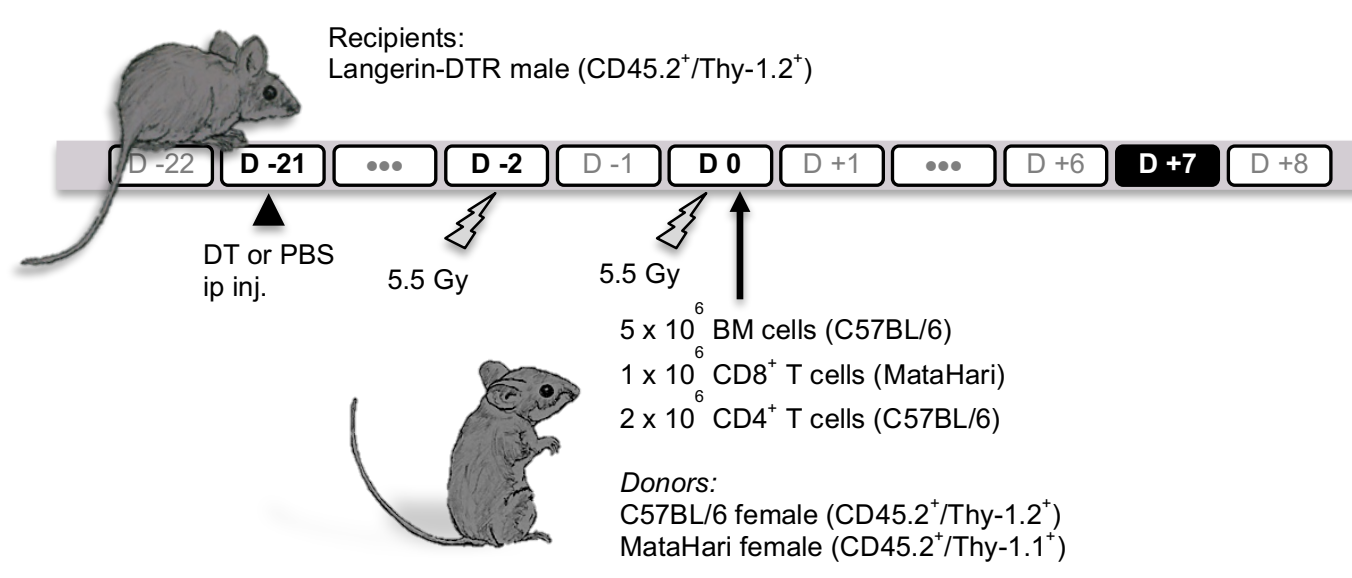

Figure 10. Adapted MataHari T cell $\rightarrow$ Langerin-DTR model.

\subsection{In vitro $\mathrm{T}$ cell activation}

In the experiments in which Ifngr $1^{-/-}$mice were used as HSCT recipients, donor $\mathrm{T}$ cells were activated in vitro prior to transfer. For this purpose, CD4 ${ }^{+}$ and $\mathrm{CD}^{+}$splenocytes were isolated as previously described and cultured overnight in complete $\mathrm{T}$ cell medium (RPMI, 1\% FCS, 1\% L-glutamine, 1\% penicillin-streptomycin, 1\% HEPES; Lonza, UK) supplemented with concanavalin A (ConA) $(2 \mu \mathrm{g} / \mathrm{ml}$, final concentration) and IL-7 (1 ng/ml, final concentration).

\subsection{Animal welfare}

All transplanted animals were kept in germ free conditions, housed in sterilised individually ventilated cages, and fed irradiated chow and acidified UV-treated water. 
Animal well-being was assessed by weight monitoring and GVHD severity evaluation of the experimental subjects'. Weight monitoring was started at the day of HSCT (D0), baseline, and all subsequent measurements were reported as percentage changes in body weight compared to baseline. Severity of GVHD was evaluated using a 6 parameter scoring system that takes into account weight loss, posture, activity, fur texture, eye opening and the presence of diarrhoea (Table 9).

Table 9. Assessment of clinical GVHD severity in transplanted subjects.

\begin{tabular}{|c|c|c|c|c|c|c|}
\hline Score & $\begin{array}{l}\text { Weight } \\
\text { loss }\end{array}$ & Posture & Activity & $\begin{array}{c}\text { Fur } \\
\text { texture }\end{array}$ & $\begin{array}{c}\text { Eye } \\
\text { opening }\end{array}$ & Diarrhoea \\
\hline 0 & $<10 \%$ & No hunch & Normal & Normal & Normal & Absent \\
\hline 0.25 & & & & & $\begin{array}{l}\text { Eyes closed } \\
\text { up to } 25 \%\end{array}$ & \\
\hline 0.5 & $\geq 10 \%$ & $\begin{array}{l}\text { Slight hunch, } \\
\text { lost on } \\
\text { movement }\end{array}$ & $\begin{array}{l}\text { Mild to moderate reduction } \\
\text { in spontaneous movement; } \\
\text { resists handling }\end{array}$ & $\begin{array}{l}\text { Angle } \\
0-45^{\circ}\end{array}$ & $\begin{array}{c}\text { Eyes closed } \\
25-50 \%\end{array}$ & \\
\hline 1 & & $\begin{array}{l}\text { Significant } \\
\text { hunch, reduced } \\
\text { on movement }\end{array}$ & $\begin{array}{l}\text { Severe reduction in } \\
\text { spontaneous movement; } \\
\text { does not resist handling }\end{array}$ & $\begin{array}{l}\text { Angle } \\
45-90^{\circ}\end{array}$ & $\begin{array}{c}\text { Eyes closed } \\
50-75 \%\end{array}$ & Present \\
\hline 1.5 & & & & & $\begin{array}{l}\text { Eyes closed } \\
75-100 \%\end{array}$ & \\
\hline 2 & & $\begin{array}{l}\text { Severe hunch, } \\
\text { persistent on } \\
\text { movement }\end{array}$ & $\begin{array}{c}\text { No spontaneous } \\
\text { movement }\end{array}$ & $\begin{array}{l}\text { Angle } \\
>90^{\circ}\end{array}$ & $\begin{array}{l}\text { Eyes } \\
\text { completely } \\
\text { closed }\end{array}$ & \\
\hline
\end{tabular}


Euthanasia of the experimental subjects was performed whenever the weight loss exceeded $20 \%$ of the baseline body weight, or the global GVHD severity score (calculated as the sum of the individual characteristics score) was equal or greater than 4.

\subsection{Treatments}

\section{a) Antibiotic prophylaxis}

All HSCT recipients received antibiotic prophylactic treatment from D-2 until the end of the experiment: enrofloxacin (Baytril ${ }^{\circledR}$; Bayer, Germany) was given in the drinking water at a final concentration of $200 \mathrm{mg} / \mathrm{ml}$.

\section{b) Lymph node lymphocyte egress blockage}

Homing trafficking blockage of activated lymphocytes from the lymph nodes to the peripheral tissues was achieved through treatment of transplanted animals with the sphingosine-1-phosphate antagonist, fingolimod (FTY720; Sigma, UK), as previously described. ${ }^{(232)}$ Briefly, HSCT recipients were injected intraperitoneally with $1.0 \mathrm{mg} / \mathrm{kg}$ of FTY720 daily, from D+3 to D+7 (experiment terminus); control subjects received an equivalent volume of saline.

\section{c) Langerhans cell depletion}

Selective depletion of epidermal Langerhans cells was achieved through treatment of Langerin-DTR mice with diphtheria toxin (DT; Sigma, UK) three weeks prior to HSCT (D-21), as previously described. ${ }^{(217)}$ Briefly: for systemic Langerhans cell depletion, animals received a single intraperitoneal injection of 
$400 \mathrm{ng}$ of DT; for localised Langerhans cell depletion, animals received intradermal injections of $25 \mathrm{ng}$ of DT in dorsal and ventral sides of the left ear (total DT dose: $50 \mathrm{ng}$ ), under general anaesthesia with $2 \%$ isoflurane with an oxygen flow rate of $1 \mathrm{~L} / \mathrm{min}$; control subjects received an equivalent volume of saline.

\section{d) Notch signalling blockage}

Blockage of Notch signalling was achieved through treatment of transplanted animals with a potent gamma secretase inhibitor, LY411575 (Sigma, UK). Animals were treated with $5 \mathrm{mg} / \mathrm{kg} /$ day of LY411575 given intraperitoneally, from $D+5$ to $D+7$ (experiment terminus); control subjects received an equivalent volume of saline.

\section{Tissue and organ harvest}

Blood samples were collected into heparinised tubes by venepuncture of the lateral tail vein, for interim analysis, or by cardiac puncture under terminal anaesthesia, at the experiment terminus.

Animals were euthanized by exposure to carbon dioxide gas in a rising concentration. Following intracardiac perfusion with $20 \mathrm{ml}$ of cold PBS to remove the blood from the vasculature, the organs of interest were harvested and stored in harvest medium (PBS, 2\% FCS, 1\% penicillin-streptomycin; Lonza, UK) on ice. Processing of the samples was started within $2 \mathrm{~h}$ from collection. 
Harvested organs included: spleen, peripheral lymph nodes (cervical, axillary, brachial and inguinal), mesenteric lymph nodes, tibias and femurs, liver, small intestine (from $0.5 \mathrm{~cm}$ below the stomach to $1 \mathrm{~cm}$ above the cecum), skin (body and ears).

\subsection{Isolation of murine immune cells}

\section{a) Lymph nodes and spleens}

Preparation of cell suspensions from spleens and lymph nodes was performed according to the laboratory's protocol, adapted from the procedure described in Current Protocols in Immunology (supplement 39). ${ }^{(233)}$ Briefly, the freshly removed organs were mashed and passed through a $40 \mu \mathrm{m}$ cell strainer; red blood cells were removed by isotonic lysis with ammonium chloride (ACK Lysing Buffer; Lonza, UK). Cells were resuspended in FACS buffer (PBS, 2\% FCS, 2 mM EDTA; Lonza UK) for counting and immunolabelling.

\section{b) Bone marrow}

Isolation of bone marrow cells was accomplished following an adaptation of the procedure described in Current Protocols in Immunology (supplement 67). ${ }^{(234)}$ Briefly, both epiphysis of the bones were cut and the bone marrow was flushed out with FACS buffer. The cell suspension was filtered through a $40 \mu \mathrm{m}$ cell strainer and red blood cells were removed by isotonic lysis with ammonium chloride. Cells were resuspended in FACS buffer for counting and immunolabelling. 


\section{c) Blood}

Erythrocytes were removed from whole blood samples by hypotonic lysis with distilled water. ${ }^{(235)}$ Cells were resuspended in FACS buffer for counting and immunolabelling.

\section{d) Liver}

An adapted version of the basic protocol described in Current Protocols in Immunology (supplement 22) ${ }^{(236)}$ was used to isolate liver lymphocytes. Briefly, the excised liver was cut into small fragments $(\sim 0.5 \mathrm{~cm}$ in diameter $)$ and incubated in digestion medium (RPMI, 2\% FCS; Lonza, UK; $200 \mathrm{U} / \mathrm{ml}$ collagenase IV; LifeTechnologies, USA; 200 U/mI DNAse I; Sigma, UK) at $37^{\circ} \mathrm{C}$ with shaking at $150 \mathrm{rpm}$ for $60 \mathrm{~min}$. The liver fragments were further dissociated and passed sequentially through a $100 \mu \mathrm{m}$ and a $40 \mu \mathrm{m}$ cell strainer. Density gradient centrifugation with Ficoll-Paque ${ }^{\mathrm{TM}}$ Plus (GE Healthcare, UK) was used for lymphocyte enrichment of the cell suspension. Cells were resuspended in FACS buffer for counting and immunolabelling.

\section{e) Small intestine}

Isolation of intraepithelial lymphocytes (IEL) and lamina propria (LP) cells from the small intestine was performed according to an adapted version of the protocol described in Current Protocols in Immunology (supplement 99). ${ }^{(237)}$ Briefly, the entire small intestine was flushed and rinsed with $40 \mathrm{ml}$ of ice cold harvest medium, and sectioned into $\sim 0.5 \mathrm{~cm}$ pieces. The intestinal pieces were incubated with detaching medium (HBSS, 5\% FCS, $1 \%$ penicillinstreptomycin, $5 \mathrm{mM}$ EDTA; Lonza, UK) at $37^{\circ} \mathrm{C}$ with shaking at $150 \mathrm{rpm}$ for 60 min. The supernatant, containing the IEL, was passed sequentially through 
a $100 \mu \mathrm{m}$ and a $40 \mu \mathrm{m}$ cell strainer and enriched for lymphocytes by density gradient centrifugation with Ficoll-Paque ${ }^{\mathrm{TM}}$ Plus. The intestinal pieces were further incubated in digestion medium at $37^{\circ} \mathrm{C}$ with shaking at $150 \mathrm{rpm}$ for $60 \mathrm{~min}$, dissociated and passed sequentially through a $100 \mu \mathrm{m}$ and a $40 \mu \mathrm{m}$ cell strainer. The cell suspension, containing the LP cells was enriched for lymphocytes by density gradient centrifugation with Ficoll-Paque ${ }^{T M}$ Plus. Cells were resuspended in FACS buffer for counting and immunolabelling.

\section{f) Skin}

Epidermal and dermal immune cells were isolated from the skin in accordance with the protocol described by Henri et al., ${ }^{(238)}$ with minor modifications. Briefly, the body skin was cut into $\sim 1 \times 1 \mathrm{~cm}$ pieces, after having been shaved and the subcutaneous fat removed, and the ears were split in two parts (ventral and dorsal). The pieces of skin were incubated overnight in dispase medium (HBSS, 2\% FCS; Lonza, UK; 2.5 mg/dl dispase II; Sigma, UK) at $4^{\circ} \mathrm{C}$, and the epidermal and dermal sheets were separated and cut into $\sim 0.5 \mathrm{~cm}$ fragments. The epidermal fragments were vortexed, mashed and passed sequentially through a $100 \mu \mathrm{m}$ and a $40 \mu \mathrm{m}$ cell strainer to disintegrate the remaining tissue and create a cell suspension. The dermal fragments were further incubated in digestion medium at $37^{\circ} \mathrm{C}$ with shaking at $150 \mathrm{rpm}$ for $60 \mathrm{~min}$, dissociated and passed through a $100 \mu \mathrm{m}$ and a $40 \mu \mathrm{m}$ cell strainer. Cells were resuspended in FACS buffer for counting and immunolabelling. 


\section{Cell counting}

Total viable cell concentration of the cell suspensions was determined using a CASY ${ }^{\circledR} 1$ Model TT cell counter and analyser system (Schärfe-System, Germany). Absolute number of specific cell populations was determined by flow cytometry using CountBright ${ }^{\mathrm{TM}}$ Absolute Counting Beads (LifeTechnologies, USA) following the manufacturer's protocol.

\section{Immunolabelling}

Cell immunolabelling was performed following the protocols described in Current Protocols in Immunology (supplement 44$)^{(239)}$ with minor adaptations. Briefly, before staining, cells were plated out at up to $1 \times 10^{6}$ cells per well in a 96 well conical bottom plate and incubated with $2.4 \mathrm{G} 2$ antibody at $4^{\circ} \mathrm{C}$ for at least 10 min to block Fc receptors. For cell surface immunolabeling, cells were incubated with the fluorochrome-conjugated antibodies (Table 10) diluted in $100 \mu \mathrm{l}$ of FACS buffer at $4^{\circ} \mathrm{C}$ for $20 \mathrm{~min}$ in the dark. For detection of $\mathrm{H} 60$ specific $\mathrm{CD}^{+} \mathrm{T}$ cells, samples were labelled with PE-conjugated pentamers for H60/H-2 $\mathrm{K}^{\mathrm{b}}$ complexes (Prolmmune, UK). When intracellular staining was required, after having performed cell surface immunolabelling, samples were washed twice with FACS buffer, fixed in $100 \mu \mathrm{l}$ of fixation solution (BD Cytofix/Cytoperm solution; BD Biosciences, UK) for $15 \mathrm{~min}$ at $4^{\circ} \mathrm{C}$ in the dark, washed twice with permeabilization solution $\left(\mathrm{BD}\right.$ Perm/Wash ${ }^{\mathrm{TM}}$ buffer; BD 
Biosciences, UK), and incubated with the fluorochrome-conjugated antibodies (Table 11) diluted in $100 \mu \mathrm{l}$ of permeabilization solution at $4^{\circ} \mathrm{C}$ for $30 \mathrm{~min}$ in the dark. For detection of cytokine production, cells were treated with brefeldin A (Sigma, UK) for $2 \mathrm{~h}$ at $37^{\circ} \mathrm{C}$, prior to immunolabelling. Samples were washed twice with FACS buffer and ressuspended in $300 \mu$ of FACS buffer for immediate analysis by flow cytometry. For dead cell exclusion, $2 \mu \mathrm{l}$ of propidium iodide (LifeTechnologies, USA) was added to the unfixed samples prior to analysis.

Table 10. Antibodies used for cell surface immunolabelling.

\begin{tabular}{|c|c|c|}
\hline Specificity & Clone & Manufacturer \\
\hline CD4 & GK1.5 & eBioscience, USA \\
\hline CD8a & $53-6.7$ & BioLegend, USA \\
\hline CD11b & $\mathrm{M} 1 / 70$ & eBioscience, USA \\
\hline CD25 & PC61 & BD Biosciences, Germany \\
\hline CD40 & HM40-3 & eBioscience, USA \\
\hline CD44 & IM7 & eBioscience, USA \\
\hline CD45 & $30-F 11$ & BioLegend, USA \\
\hline CD45.1 & A20 & BD Biosciences, Germany \\
\hline CD45.2 & 104 & BD Biosciences, Germany \\
\hline CD62L & MEL-14 & BD Biosciences, Germany \\
\hline CD69 & $\mathrm{H} 1.2 \mathrm{~F} 3$ & eBioscience, USA \\
\hline CD70 & FR70 & eBioscience, USA \\
\hline CD86 (B7-2) & GL1 & eBioscience, USA \\
\hline CD90.1 (Thy-1.1) & HIS51 & eBioscience, USA \\
\hline CD90.2 (Thy-1.2) & $53-2.1$ & BD Biosciences, Germany \\
\hline CD103 & M290 & BD Biosciences, Germany \\
\hline CD326 (EpCAM) & G8.8 & eBioscience, USA \\
\hline MHC class I $\left(H-2 K^{d}\right)$ & SF1-1.1 & BD Biosciences, Germany \\
\hline MHC class II (I-A/I-E) & M5/114.15.2 & eBioscience, USA \\
\hline Yठ TCR & eBioGL3 (GL-3, GL3) & eBioscience, USA \\
\hline V $\beta$ 8.3 TCR & 1B3.3 & BD Biosciences, Germany \\
\hline
\end{tabular}


Table 11. Antibodies used for intracellular immunolabelling.

\begin{tabular}{lll}
\hline Specificity & Clone & Manufacturer \\
\hline \hline Active Caspase-3 & C92-605 & BD Biosciences, Germany \\
CD207 (Langerin) & eBioL31 & eBioscience, USA \\
IFN-y & XMG1.2 & BD Biosciences, Germany \\
TNF- $\alpha$ & MP6-XT22 & BD Biosciences, Germany \\
\hline
\end{tabular}

Fluorescence minus one controls and isotype controls were used to accurately discriminate positive versus negative signals, and the gating strategy was established accordingly.

\section{Flow cytometry}

Multicolour flow cytometry data acquisition was done with BD LSRFortessa and BD LSR II cell analysers equipped with BD FACSDiva v6.2 software (BD Biosciences, Germany).

Fluorescence activated cell sorting was performed on a BD FACSAria equipped with BD FACSDiva v5.0.3 software (BD Biosciences, Germany). All samples were maintained at $4^{\circ} \mathrm{C}$ for the duration of the sort. Three biological replicates were obtained for every tissue, each containing a minimum of 5000 cells (2-5 animals samples were pooled when necessary).

Sort purity was accessed for all samples and only those with purity $\geq 95 \%$ were used for RNA extraction. Cells were sorted directly into Buffer RLT (Qiagen, USA) with 1\% $\beta$-mercaptoethanol (Sigma, UK), disrupted 
through vortexing at $3200 \mathrm{rpm}$ for $1 \mathrm{~min}$, and immediately stored at $-80^{\circ} \mathrm{C}$ until further processing.

Flow cytometry data were analysed with FlowJo X v10 (Tree Star, Inc, USA).

\section{Sample preparation for gene expression}

RNA was extracted using the RNeasy Micro Kit (Qiagen, USA) following the manufacturer's protocol. RNA yield, quality and integrity were evaluated using the RNA 6000 Pico LabChip kit on an Agilent 2100 Bioanalyser (Agilent Technologies, USA).

For microarray studies, the Ovation Pico WTA System V2 kit (NuGEN, USA) was used to prepare amplified cDNA from total RNA for fragmentation and labeling using the Encore Biotin Module kit (NuGEN, USA), according to kit instructions, and then hybridized onto GeneChip Mouse Gene 2.0 ST arrays (Affymetrix, USA).

For RNA-seq studies, RNA samples were amplifyed using the SMART-Seq ${ }^{\circledR}$ v4 Ultra ${ }^{\circledR}$ Low Input RNA Kit. Paired-end sequencing libraries were prepared from the amplified cDNA according to the Nextera ${ }^{\circledR}$ XT DNA library prep protocol, and sequenced using an Illumina NextSeq 500 (Illumina, USA) (38 base-paired reads).

Microarray and RNA-seq studies were performed in collaboration with UCL Genomics. 


\section{Microarray analysis}

\subsection{Data acquisition and processing}

Hybridized arrays were scanned with a GeneChip $30007 \mathrm{G}$ scanner (Affymetrix, USA) and the image data processed to generate .cel files. Expression Console Software, version 1.4.1 (Affymetrix, USA) was used to generate quality control statistics for each sample and the internal reproducibility was assessed by calculating the Pearson correlation coefficient for the pairs of biological replicates for each tissue (Appendix Supplementary Figure 1); samples with $r<0.9$ were considered outliers and were excluded from the analysis $(\mathrm{B} 6 \rightarrow 129$ model: mesenteric lymph nodes replicate 1; MataHari T cell model: blood $\alpha 4 \beta 7^{+}$- replicate 1 , bone marrow replicate 2, gut lamina propria - replicate 1 .

Raw sample expression signals were background subtracted, quantile normalized, and the probe level data were summarized using the Robust Multi-array Average algorithm;(240-242) implemented in the oligo BioConductor R package. ${ }^{(243)}$ The ComBat algorithm ${ }^{(244)}$ from the sva BioConductor $\mathrm{R}$ package ${ }^{(245)}$ was employed to adjust for batch effects. Transcripts identified through multiple probes were collapsed based on maximum expression values using the CollapseDataset module of GenePattern software (Broad Institute). ${ }^{(246)}$ To enhance statistical testing power, contaminant non-T cell genes were excluded from analysis by filtering out transcripts identified as 
tissue-specific (for liver, small intestine and skin) in the PaGenBase database (Appendix - Supplementary Table 1). ${ }^{(247)}$

\subsection{Samples relationship visualisation}

Multivariate statistical analysis methods implemented in the stats $\mathrm{R}$ package, ${ }^{(248)}$ in particular principal components analysis and multidimensional scaling, were applied to perform dimensionality reduction of the datasets and visualization of the samples relationships.

Similarities between groups were evaluated by unsupervised hierarchical clustering analysis, using average-linkage criteria and Pearson correlation-based dissimilarity matrix; the validity and stability of the clusters was assess by random sampling with replacement of the genes using non-parametric bootstrap methodology implemented in the pvclust CRAN R package..$^{(249,250)}$

Additional hierarchical clustering and heat maps were produced using the matrix visualization and analysis platform GENE-E (Broad Institute, USA). ${ }^{(251)}$

\subsection{Differential gene expression}

The limma BioConductor R package ${ }^{(252)}$ was used to perform analyses of gene differential expression, using an empirical Bayes moderated t-statistic, ${ }^{(253)}$ with a cut-off of 0.05 , corrected for multiple hypothesis testing using Benjamini-Hochberg procedure and an absolute fold-change cut-off $>2.0$. 


\subsection{Gene set enrichment analysis (GSEA)}

Gene Set Enrichment Analysis was performed as previously described, ${ }^{(254)}$ using the GSEA software ${ }^{(255)}$ with the curated gene sets (C2) from the KEGG pathway database (Kyoto Encyclopedia of Genes and Genomes), ${ }^{(256,257)}$ the gene sets derived from the Biological Process Ontology database (C5) defined by the Gene Ontology Consortium, ${ }^{(258,259)}$ collected in the Molecular Signatures Database (MSigDB v5.1), and the gene sets derived from the modules identified by WGCNA in the MataHari T cell data set.

\subsection{Weighted gene co-expression network analysis (WGCNA)}

Scale-free network topology analysis of microarray expression data was performed using the WGCNA R package, as previously described. ${ }^{(229,260)} \mathrm{A}$ signed hybrid weighted correlation network was constructed using a Pearson correlation matrix created from the pairwise comparison between all pairs of genes, and a soft thresholding power $\beta=8$. The topological overlap was calculated as a measure of network interconnectedness, and genes were grouped by average linkage hierarchical clustering on the basis of the topological overlap dissimilarity (1-topological overlap). Module eigengenes were calculated using a dynamic tree-cutting algorithm and merging threshold function at 0.25 . The modules identified were correlated to the sample traits using a binary vector representation of the tissues of origin and study groups. 
To validate the microarray analysis, preservation of the WGCNA modules identified in the MataHari model dataset was tested against the dataset of the $B 6 \rightarrow 129$ model, using the R function "modulePreservation" in the WGCNA R package, as previously described. ${ }^{(261)}$ Results were interpreted according to the following thresholds for $Z_{\text {summary: if }} Z_{\text {summary }} \geq 10$, module "strongly preserved"; if $2 \leq Z_{\text {summary }}<10$, module "weak to moderately preserved"; if $Z_{\text {summary }}<2$, module "not preserved". Significance of the $Z_{\text {summary }}$ scores was calculated by permutation analysis.

Intramodular hub genes, which are genes that have the highest number of connections within a module, were identified on the basis of having eigengene-based connectivity $(\mathrm{kME})>0.8$ and gene significance $(\mathrm{GS})>0.2$. Visualisation of the modules network of gene connections was accomplished with the Cytoscape v3.4 software. ${ }^{(262)}$

\subsection{Gene ontology (GO) overrepresentation analysis}

The Web-based Gene Set Analysis Toolkit (WebGestalt), ${ }^{(263,264)}$ a suite of tools for functional enrichment analysis, was used to identify overrepresented GO annotation categories and translate gene lists into functional profiles. Enrichment of GO terms and associated p-values were calculated based on hypergeometric distribution statistics, adjusting the false discovery rate using the Benjamini-Hochberg procedure. 


\section{RNA-seq analysis}

\subsection{Library preprocessing}

FASTQ Toolkit, version 1.0.0 (BaseSpace, Illumina), was used for adapter trimming of the reads in order to eliminate poor quality bases occurring at the ends of reads which could lead to erroneous read alignment. Read alignment was performed using TopHat Alignment, version 1.0.0 (BaseSpace, Illumina) by mapping the RNA-seq reads to the human genome. Transcripts were identified using Cufflinks Assembly \& DE, version 2.0.0 (BaseSpace, Illumina), based on the Homo sapiens hg38 RefSeq gene annotations.

\subsection{Calculation of read-level metrics}

Gene expression levels (expressed in Reads Per Kilobase per Million, RPKM) were calculated using the Cufflinks Assembly \& DE, version 2.0.0 (BaseSpace, Illumina), employing a geometric library normalization method and a fragment bias correction algorithm, which re-scales gene counts to correct for differences in both library sizes and gene length.

\subsection{Single-sample GSEA}

Single-sample GSEA was performed using the ssGSEAProjection (v4) module of GenePattern software (Broad Institute), ${ }^{(246)}$ following the protocol 
described by Barbie et al., ${ }^{(265)}$ using the modules identified by WGCNA in the MataHari T cell data set as gene sets. For this purpose the human orthologs for the mouse genes were using the MGI-Mouse Vertebrate Homology Database.

\section{Statistical analysis}

Apart from microarray and RNA-seq data, which were analysed with the aforementioned programs and methodologies, statistical analysis was performed using GraphPad Prism version 6.00 for Mac OsX (GraphPad Software, USA). ${ }^{(266)}$

Survival curves were created using the Kaplan-Meier method.

The data are expressed as mean \pm standard deviation. Significance was assessed using a student's t-test, 1-way ANOVA or 2-way ANOVA with Sidak's post hoc test. A p-value of less than 0.05 was taken to indicate a significant difference between groups; only statistically significant differences are marked in the figures. Sample sizes, n, are indicated in the figure legends. 
III. Results 


\section{Transcription profiles of donor-derived $\mathrm{T}_{\text {eff }}$ cells are different between SLO and GVHD target organs}

In order to test the hypothesis that GVHD target tissues exert dominant, idiosyncratic roles in regulating the effector function of alloreactive $\mathrm{CD} 8^{+} \mathrm{T}$ cells, two murine models of acute GVHD were studied: (1) B6 $\rightarrow 129$ model; ${ }^{(267,268)}$ (2) MataHari T cell model. ${ }^{(118)}$ The models used were selected for their clinical relevance, as they are both miHA-mismatched models that reproduce the clinical features and histological abnormalities found in patients with acute GVHD. The former is used as a model of HLA-identical sibling HSCT, and the latter simulates $\mathrm{F} \rightarrow \mathrm{M}$ HLA-identical transplant, which is associated with higher risk of GVHD.

In the $\mathrm{B} 6 \rightarrow 129$ model, the allogeneic immune reaction is mediated by a polyclonal T cell response against multiple antigens. ${ }^{(267,268)}$ This model mirrors the clinical setting of the majority of the HLA-matched HSCT, and was used to access the diversity of genetic signatures between the different organs. Since it has been described that $\mathrm{H} 60$ is an immunodominant miHA in the $\mathrm{B} 6 \rightarrow 129$ strain combination, ${ }^{(197)}$ characterisation of the ratio of $\mathrm{H} 60$-specific $\mathrm{CD}^{+}{ }^{+} \mathrm{T}$ cells was performed for each tissue to evaluate its impact on the variety of transcription profiles. 
In order to evaluate the extent to which distinct transcriptional profiles are a result of different TCR repertoire or reflect contrasting tissue antigen distribution, the MataHari $\mathrm{T}$ cell model was employed. In this model the donor-recipient mismatch is restricted to a single gender-specific ubiquitously expressed antigen, the male UTY peptide, and GVHD pathology is mediated by a monoclonal population of $\mathrm{CD}^{+} \mathrm{T}$ cells expressing a transgenic TCR specific for $\mathrm{H}-2 \mathrm{D}^{\mathrm{b}}$ :Uty. ${ }^{(118)}$

To ensure a global approach to the characterisation of donor $\mathrm{CD}^{+} \mathrm{T}$ cell programs during GVHD development, a whole-transcriptome profiling platform was chosen. In contrast with classical 3' expression microarrays which use the expression at the 3 ' end to approximate expression of the entire gene and are thus unable to discriminate between alternatively spliced transcripts that have identical 3' ends, whole-transcriptome approaches enable the detection not only of the level of expression, but also precisely what is being expressed, including alternative isoforms or genomic deletions. Affymetrix GeneChip $®$ Mouse Gene 2.0 ST Array provides a comprehensive transcriptome coverage identifying over 30000 coding and non-coding transcripts.

In this first results chapter, classical methods of microarray analysis were employed to characterise the broad changes to the transcriptional profile of donor $\mathrm{CD}^{+} \mathrm{T}$ cells occurring during the development of acute GVHD. 


\subsection{Brief characterisation of the minor histocompatibility antigen mismatched models}

\section{a) $B 6 \rightarrow 129$ model}

The $\mathrm{B} 6 \rightarrow 129$ is a moderate to severe multiple mHA murine GVHD model, achieved by the transplant of C57BL/6 bone marrow and T cells to lethally irradiated 129/Sv recipients (Chapter II-Materials \& Methods, Figure 8). The onset of the manifestations of GVHD was observed at day 3 - 4 and peaked at day 7 post-transplant (Figure 11-A). Intestinal involvement was one of the major features of this model, denoted by persistent diarrhoea. Consequently, a significant decrease in body weight was registered in the first week post-transplant (Figure 11-B), which was never recovered in the $\mathrm{BM}+\mathrm{T}$ cells group, in clear contrast to the BM only control group. Other manifestations of acute GVHD recorded were hair ruffling, back hunching and lowered mobility. The high early mortality observed (Figure 11-C) reflects the severity and the rapid progression of the disease, which required the euthanasia of over half of the $\mathrm{BM}+\mathrm{T}$ cell recipients by the end of the first week post-transplant.

Based on the GVHD kinetics, day 6 post-transplant was elected as the ideal time point for the collection of tissues samples for isolation of infiltrating lymphocytes. In accordance with the clinical features, the GVHD target organ with the highest proportion of donor derived $\mathrm{CD}^{+} \mathrm{T}$ cells from infiltrating 
lymphocytes was the small intestine, in particular in the intraepithelial compartment, followed by the skin and the liver (Figure 12).

It has been reported that in a wide variety of multiple mHA mismatch GVHD models, the cytotoxic effectors generated were specific for a small fraction of the non-H-2 $\mathrm{H}$ antigens. ${ }^{(269)}$ Choi and colleagues ${ }^{(197,270)}$ have demonstrated that in the $\mathrm{B} 6 \rightarrow 129$ model the $\mathrm{H} 60$ antigen is immunodominant, accounting for the specificity of a fifth of the expanded donor $\mathrm{CD}^{+} \mathrm{T}$ cells. To better characterise this effect and assess its impact on the diversity of the alloreactive immune response at each site, samples of lymphocytes isolated from the lymph nodes, spleen, blood, liver, small intestine and skin were analysed by flow cytometry using PE-conjugated pentamers for $\mathrm{H} 60 / \mathrm{H}-2 \mathrm{~K}^{\mathrm{b}}$ complexes (Figure 13). Overall, at day 6 post-transplant, the frequency of $\mathrm{H} 60$ pentamer positive donor derived $\mathrm{CD}^{+} \mathrm{T}$ cells was less then $7 \%$, with the exception of the gut lamina propria and the blood where it amounted to $9.4 \%$ and $11.7 \%$, respectively (Figure 13-B and C). These results show that although $\mathrm{H} 60$ is a dominant $\mathrm{mHA}$, it did not preclude the generation of CTL against other mHA; thus, gene expression profiling of such samples should still reflect the diversity of a polyclonal immune reaction against an array of antigens.

\section{b) MataHari T cell model}

The MataHari T cell model was characterised by Dr. Thomas Conlan, as part of his PhD project. ${ }^{(217)}$ Briefly, this is a model of a female into male mHA mismatch HSCT, in which a monoclonal population of transgenic $\mathrm{CD}^{+} \mathrm{T}$ cells that recognise the $D^{b}$ :Uty peptide (MataHari T cells) are transferred together 
with bone marrow and polyclonal $\mathrm{CD}^{+} \mathrm{T}$ cells from wild-type $\mathrm{C} 57 \mathrm{BL} / 6$ female into irradiated wild-type male C57BL/6 recipients (Chapter II-Materials \& Methods, Figure 9). Since the HY antigen is a ubiquitously expressed male-specific antigen, the acute GVHD immune reaction only develops in the male recipients.

This model has been described as of mild to moderate severity, affecting predominantly the skin, lung, small intestine and the liver (Figure 14). Similarly to the $B 6 \rightarrow 129$ model, the onset of the clinical signs of GVHD is at day 4 - 5 post-transplant, and it progresses gradually for 2 - 3 weeks (Figure 15-A and B), with 50\% mortality at 4 weeks post-transplant (Figure 15-C).

To maintain uniformity within the study, samples were collected at day 7 post-transplant. Interestingly, even though at this stage the clinical manifestations of acute GVHD were less severe than those observed in the B6 $\rightarrow 129$ model, the majority of the organs showed a greater lymphocyte infiltration (Figure 16). 
A

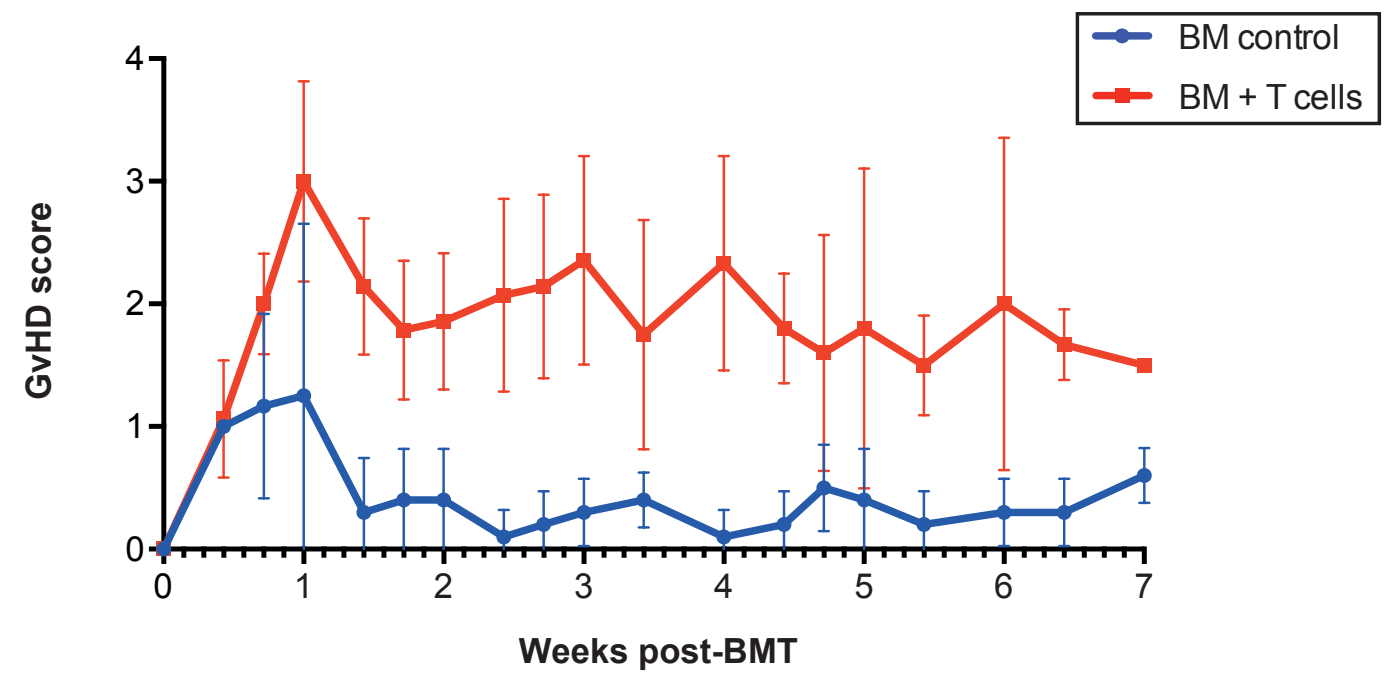

B

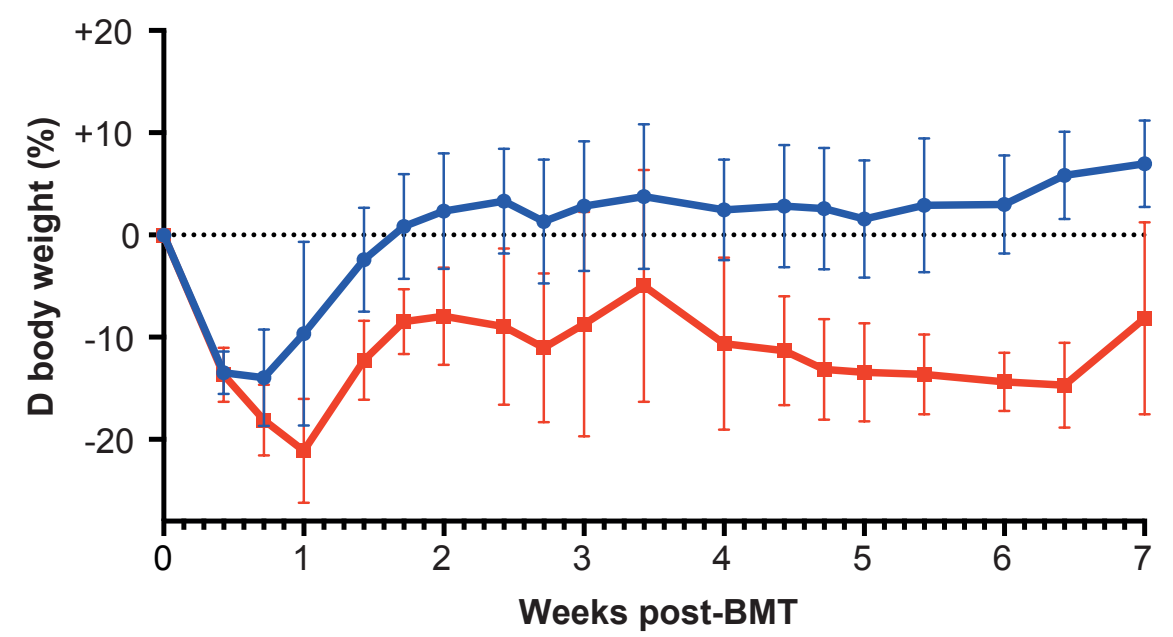

C

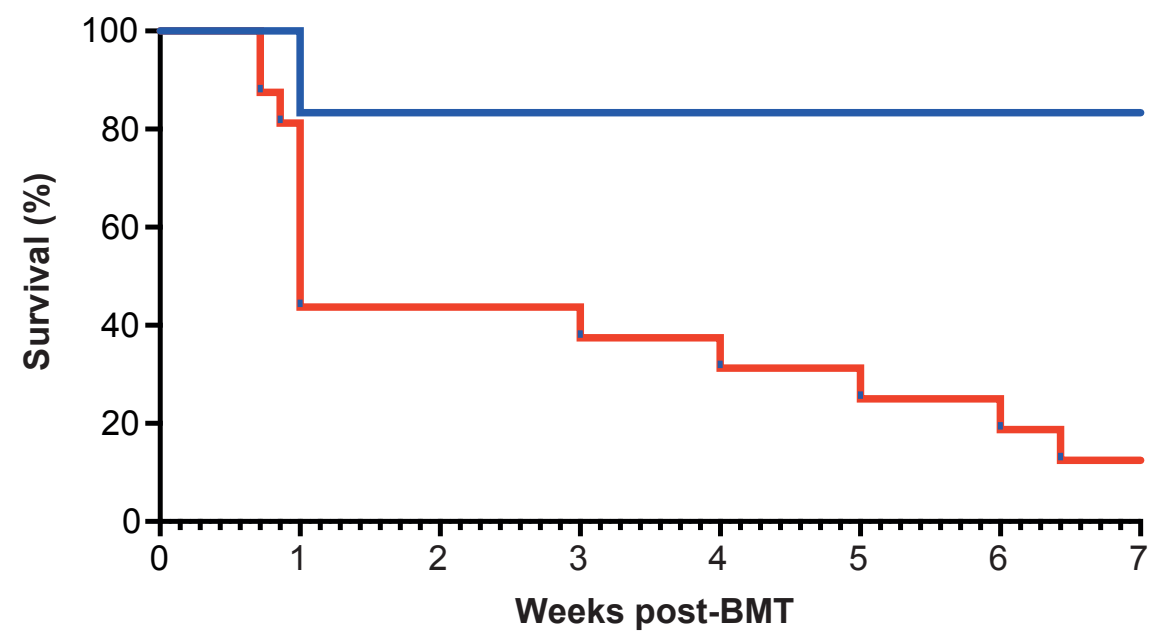

Figure 11. Characterisation of the $B 6 \rightarrow 129$ model: (A) GVHD score; (B) percentage of body weight change; (C) survival. Evaluation of the GVHD severity was performed by assessment of the subjects' global wellbeing through a standardised clinical score $(A)$, monitoring of the body weight $(B)$, and appraisal of the mortality $(C)$. Plots $A$ and $B$ represent the mean values and standard deviations measured at specific time-points over 7 weeks; plot $C$ is a Kaplan-Meyer survival plot showing the survival rates over time (blue line: $B M$ only recipient controls, $n=6$; red line: $B M+T$ cells recipients, $n=16$ ). The $B 6 \rightarrow 129$ is a moderate to severe GVHD model, with an early onset of GVHD manifestations and high lethality after the first week post-transplant. $\mathrm{BM}$, bone marrow 
A

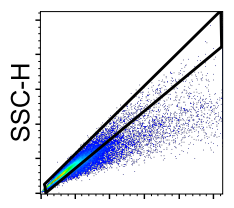

SSC-A

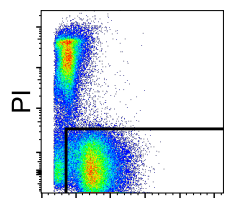

FSC-A

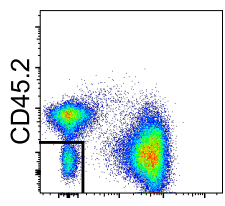

CD45.1

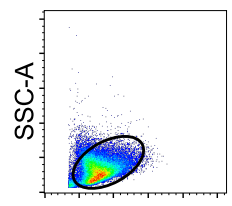

FSC-A

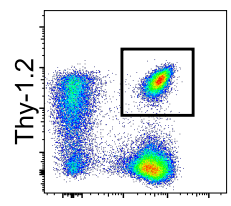

CD45.1
B
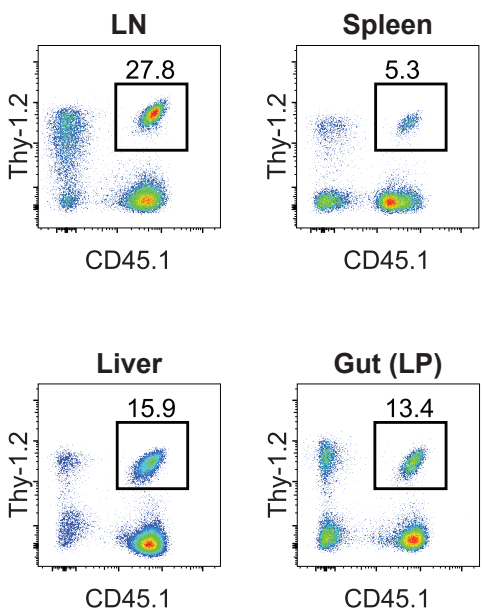

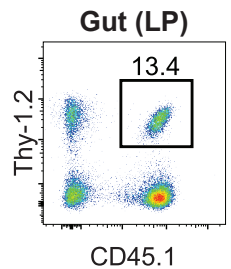

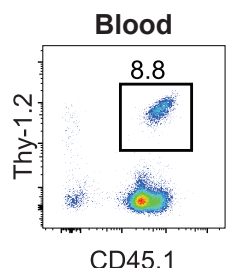

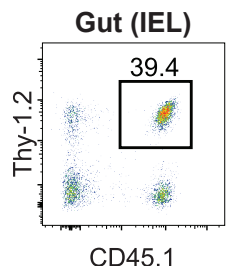

C

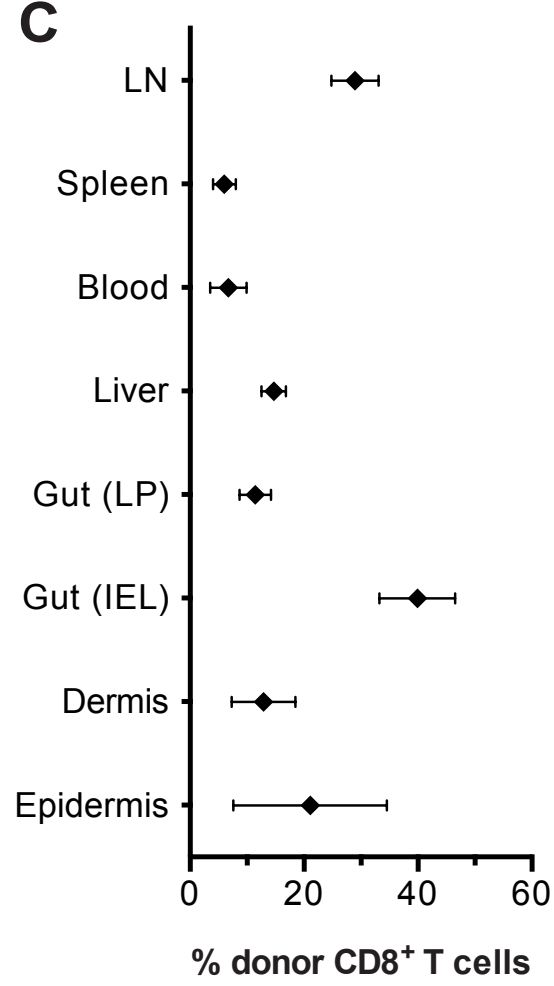

Figure 12. Donor $\mathrm{CD} 8^{+} \mathrm{T}$ cell infiltration of the secondary lymphoid organs and GVHD target organs at day 6 post-transplant in the B6 $\rightarrow 129$ model. (A) Gating strategy used to identify the donor $\mathrm{CD}^{+}{ }^{+}$T cells: exclusion of doublets $\rightarrow$ exclusion of propidium iodide positive cells $\rightarrow$ exclusion of stroma cells $\rightarrow$ morphologic lymphocyte selection $\rightarrow$ donor $\mathrm{CD} 8^{+}$T cells selection based on expression of congenic markers. (B) Representative plots showing the percentage from lymphocytes of donor $\mathrm{CD} 8^{+} \mathrm{T}$ cells in each organ. (C) Summary data: mean values and standard deviations of the percentage from lymphocytes of donor $\mathrm{CD} 8^{+} \mathrm{T}$ cells are plotted for each tissue ( $n=4-11$; data pooled from 4 independent experiments). LN, lymph nodes; LP, lamina propria; IEL, intraepithelial lymphocytes. 
A

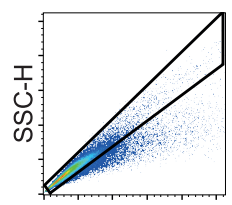

SSC-A

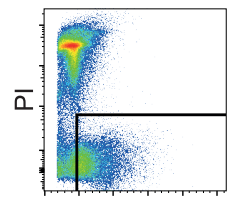

FSC-A

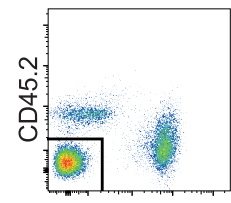

CD45.1

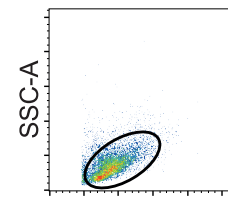

FSC-A

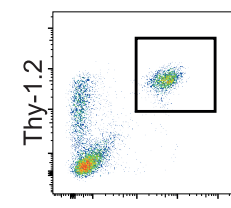

CD8
B
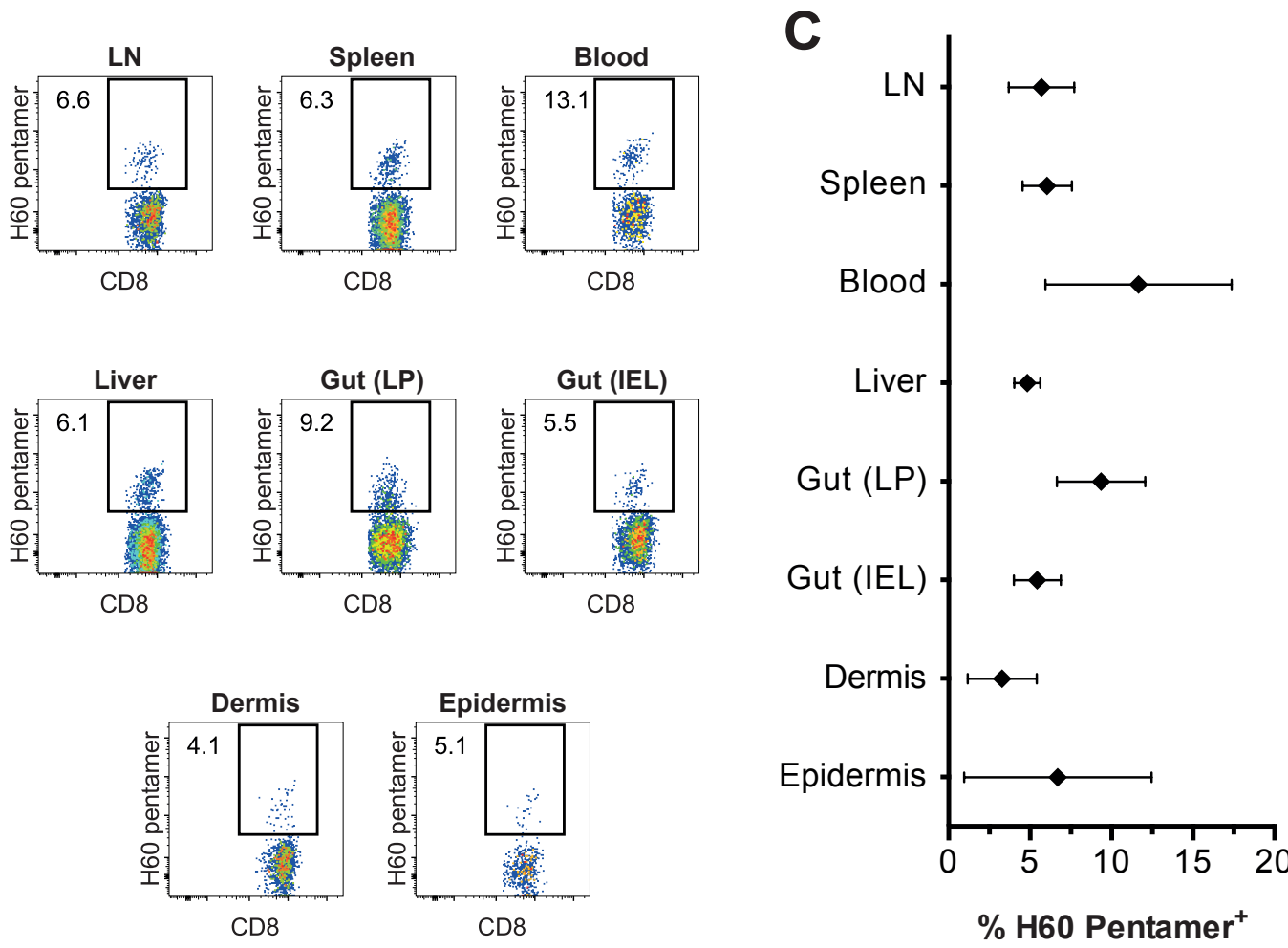

Figure 13. Frequency of $\mathrm{H} 60$-specific from tissue infiltrating donor $\mathrm{CD} 8^{+} \mathrm{T}$ cells in thr $\mathrm{B} 6 \rightarrow 129$ model. (A) Gating strategy used to identify the donor $\mathrm{CD}^{+} \mathrm{T}$ cells: exclusion of doublets $\rightarrow$ exclusion of propidium iodide positive cells $\rightarrow$ exclusion of stroma cells $\rightarrow$ morphologic lymphocyte selection $\rightarrow$ donor $\mathrm{CD}^{+}{ }^{\top}$ cells selection based on expression of congenic markers. (B) Representative plots showing the percentage $\mathrm{H} 60$-pentamer ${ }^{+}$cells from donor $\mathrm{CD}^{+} \mathrm{T}$ cells, in each organ. (C) Summary data: mean values and standard deviations of the percentage of H60-pentamer ${ }^{+}$cells from donor $\mathrm{CD}^{+} \mathrm{T}$ cells are plotted for each tissue $(n=4-8$; data pooled from 2 independent experiments). Although $\mathrm{H} 60$ behaves as a dominant $\mathrm{mHA}$, it accounts for less that $10 \%$ of the diversity of donor $\mathrm{CD}^{+} \mathrm{T}$ cells that infiltrate most of the tissues. LN, lymph nodes; LP, lamina propria; IEL, intraepithelial lymphocytes. 


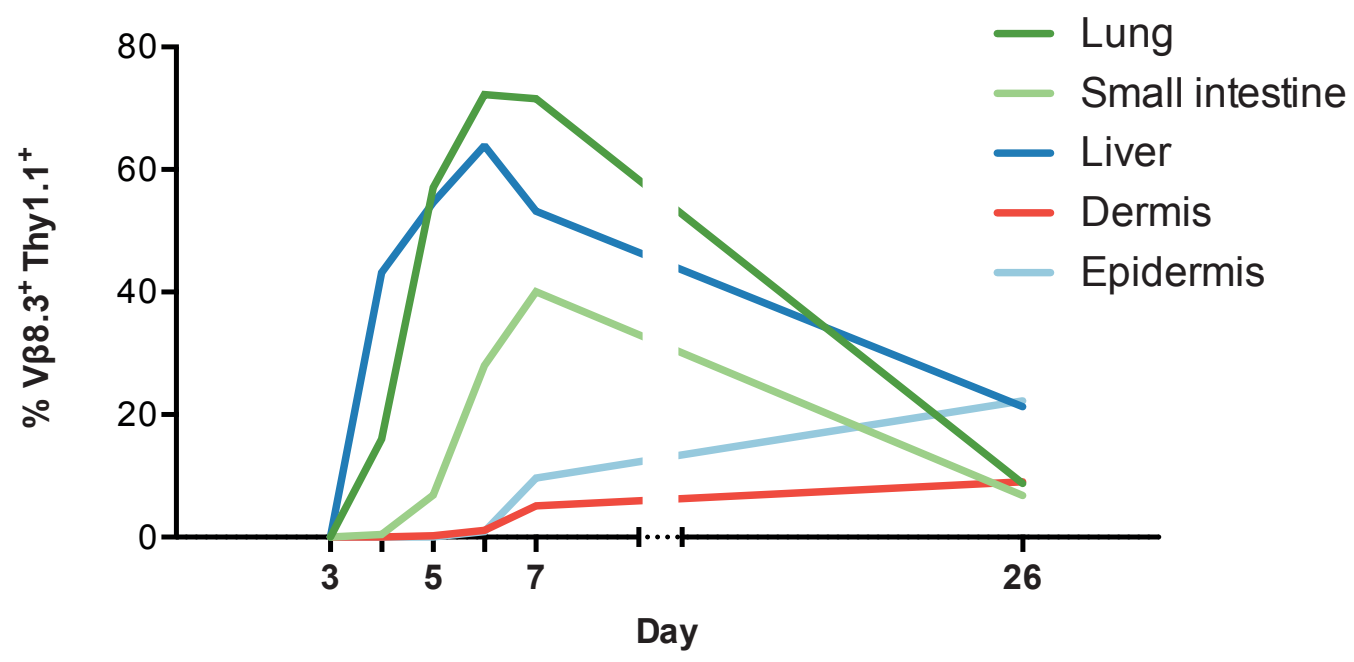

Figure 14. Kinetics of MataHari T cell infiltration of the GVHD target organs.

Courtesy of Dr Thomas Conlan..$^{(217)}$ 
A

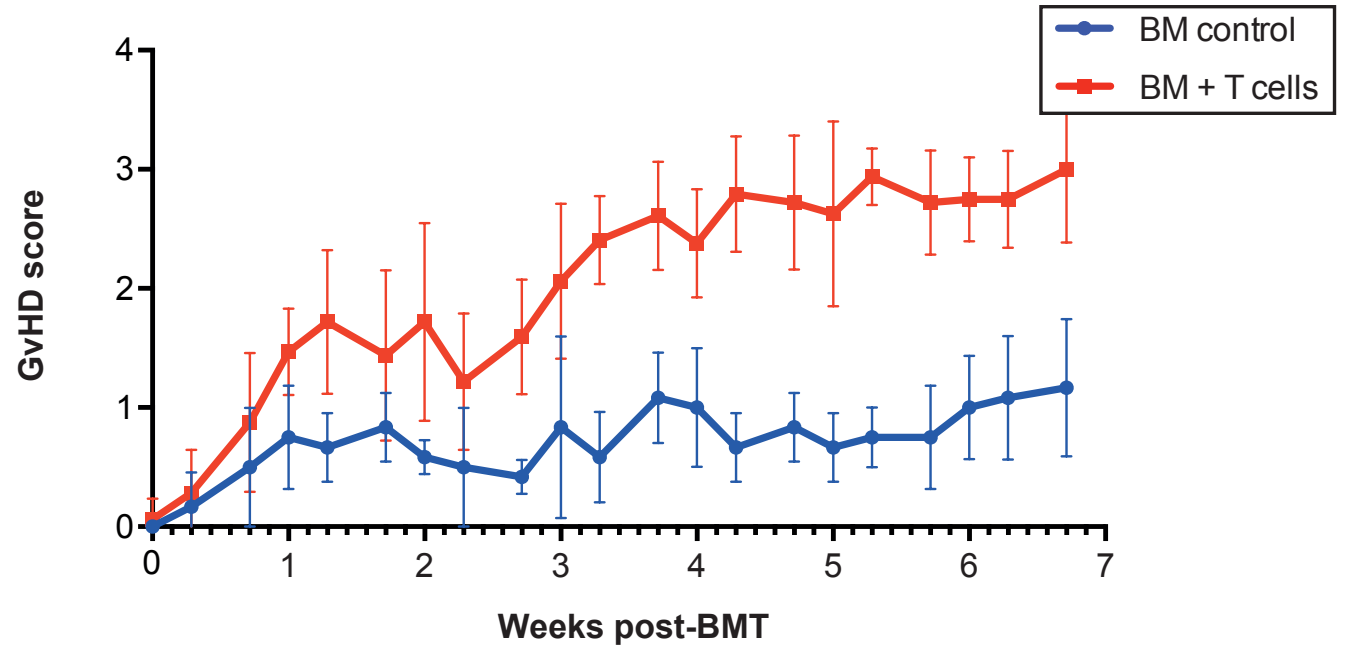

B

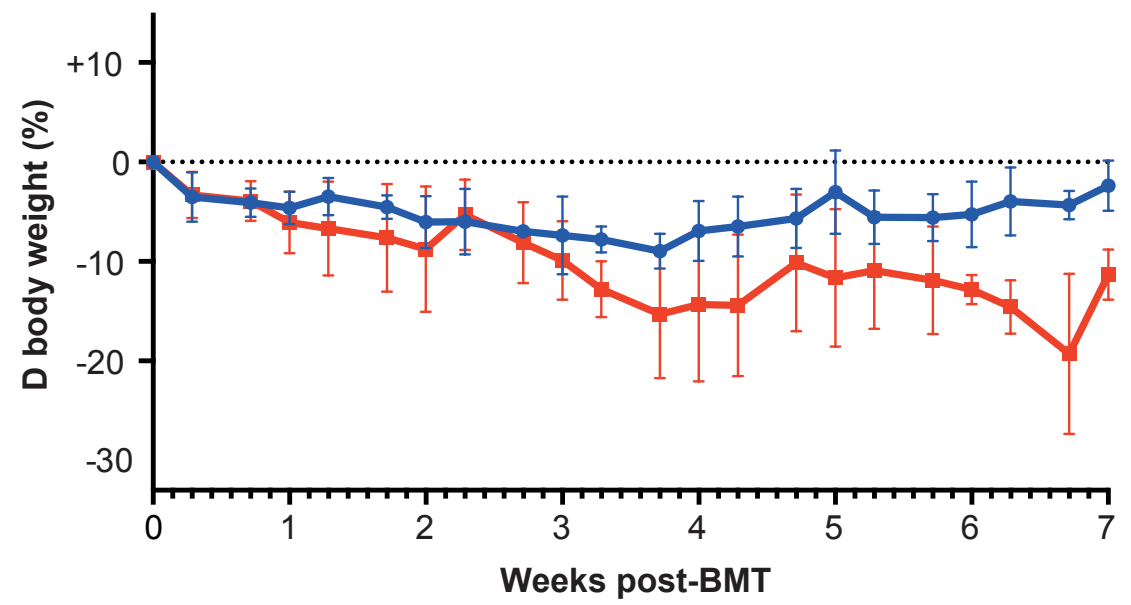

C

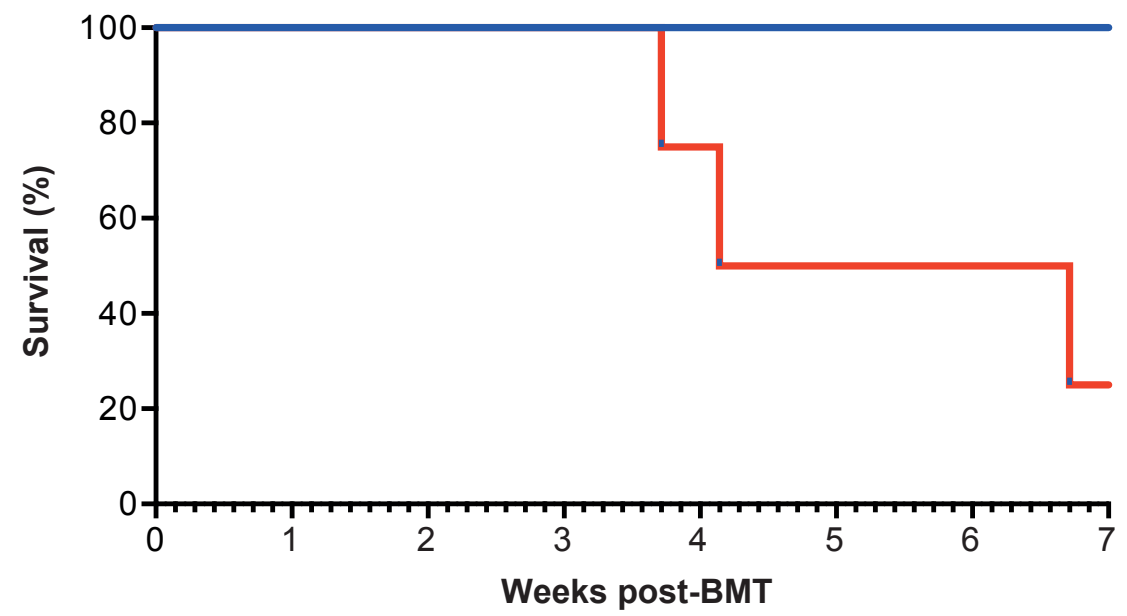

Figure 15. Characterisation of the MataHari T cell model: (A) GVHD score; (B) percentage of body weight change; (C) survival. General wellbeing of transplanted animals assessed by a standardised clinical score $(A)$, body weight $(B)$, and mortality $(C)$ records were used to determine GVHD severity. Plots $A$ and $B$ represent the mean values and standard deviations measured at specific time-points over 7 weeks; plot $C$ is a Kaplan-Meyer survival plot showing the survival rates over time (blue line: $B M$ only recipient controls, $n=3$; red line: $B M+T$ cells recipients, $n=8$ ). The MataHari T cell model is a mild to moderate GVHD model, when compared to the $\mathrm{B} 6 \rightarrow 129$ model, characterised by a gradual progression of clinical manifestations over the course of $3-4$ weeks.

Courtesy of Dr Thomas Conlan. ${ }^{(217)}$ 
A

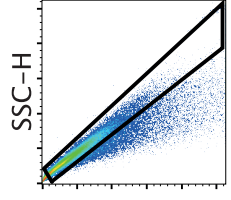

SSC-A

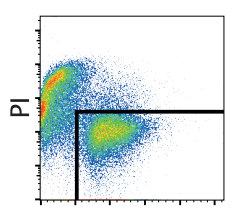

FSC-A

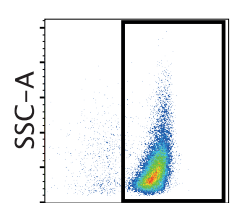

CD45.2

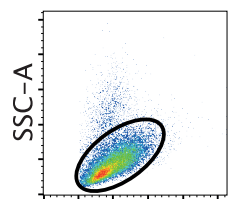

FSC-A

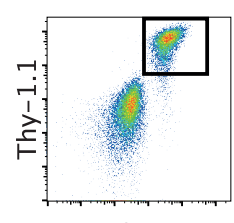

V $\beta 8.3$
B

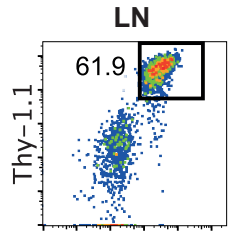

V $\beta 8.3$

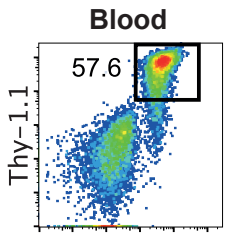

V $\beta 8.3$

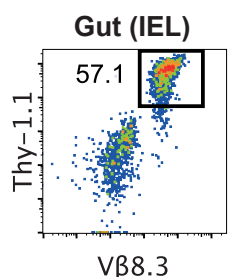

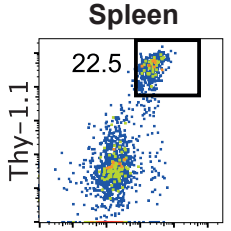

V $\beta 8.3$

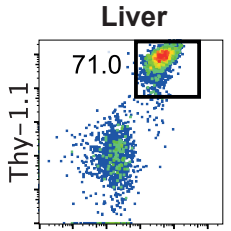

V $\beta 8.3$

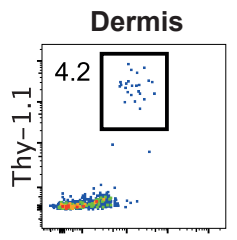

V $\beta 8.3$

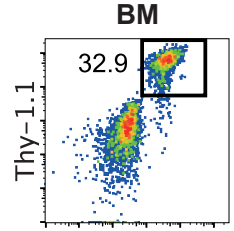

V $\beta 8.3$

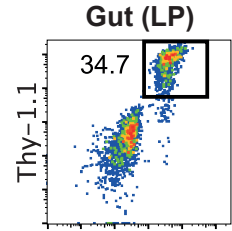

V $\beta 8.3$

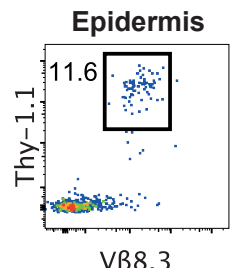

C

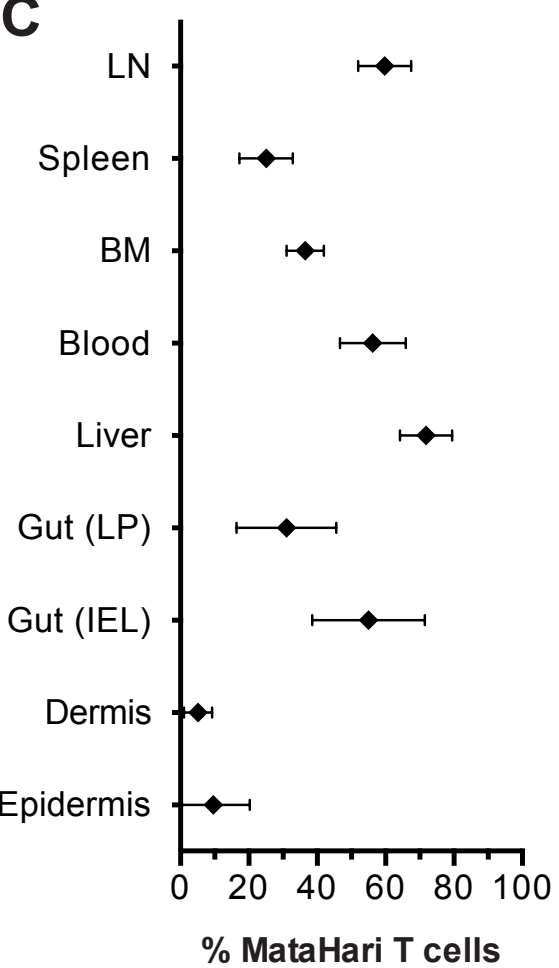

Figure 16. Donor $\mathrm{CD}^{+}$(MataHari) T cell infiltration of the secondary lymphoid organs and GVHD target organs at day 7 post-transplant. (A) Gating strategy used to identify the donor $\mathrm{CD}^{+} \mathrm{T}$ cells: exclusion of doublets $\rightarrow$ exclusion of propidium iodide positive cells $\rightarrow$ exclusion of stroma cells $\rightarrow$ morphologic lymphocyte selection $\rightarrow$ donor $\mathrm{CD}^{+} \mathrm{T}$ cells selection based on expression of congenic markers. (B) Representative plots showing the percentage from lymphocytes of donor $\mathrm{CD}^{+} \mathrm{T}$ cells in each organ. (C) Summary data: mean values and standard deviations of the percentage from lymphocytes of donor $\mathrm{CD}^{+} \mathrm{T}$ cells are plotted for each tissue ( $n=6$; data pooled from 2 independent experiments). Organ infiltration by donor CD8+ T cell exhibits a similar patter to that of the B6 $\rightarrow 129$ model. $L N$, lymph nodes; BM, bone marrow; LP, lamina propria; IEL, intraepithelial lymphocytes. 


\subsection{Purification and microarray analysis of donor derived $\mathrm{CDB}^{+} \mathrm{T}$ cells}

All samples used in this project were collected at day 6 (for the $\mathrm{B} 6 \rightarrow 129$ model) or day 7 (for the MataHari $\mathrm{T}$ cell model) post-transplant, processed and stored using optimized protocols designed to standardize the cell isolation methodology, reduce the processing time and maximize the cell sorting purity, in order to reproducibly yield sufficient quantities of cells to ultimately generate the required amount of high quality RNA.

To obtain precise expression profiles, positive selection of viable donor derived $\mathrm{CD}^{+} \mathrm{T}$ cells was performed through FACS sorting. Examples of the gating strategy employed for cell sorting in each model are illustrated in Figure 17: (1) exclusion of doublets, (2) exclusion of propidium iodide positive cells, (3) exclusion of stroma cells, (4) morphologic lymphocyte selection, (5) selection of donor $\mathrm{CD}^{+} \mathrm{T}$ cells based on the expression of congenic markers, (6) confirmation of CD8 positivity.

In order to ensure the comparability between groups, in particular in the secondary lymphoid organ samples, the donor $\mathrm{CD}^{+} \mathrm{T}$ cells were CFSE labelled prior to infusion into the recipients (both syngeneic and allogeneic). By only sorting CFSE low cells it was ensured that the microarray analysis was 
restricted to cells that had undergone equivalent number of divisions (Figure 18-A).

In respect to the blood samples, staining for the $\alpha 4 \beta 7$ integrin (LPAM-1) permitted the differential sorting of cells imprinted for gut homing (CFSE $^{\text {low }}$ LPAM-1 $1^{\text {high }}$ vs CFSE ${ }^{\text {low }}$ LPAM-1 ${ }^{\text {low }}$ ) (Figure 18-B).

Purity of isolated populations was measured as the percentage CD8 ${ }^{+}$ cells bearing the donor congenic markers (Figure 19-A), which in both models was greater than 98\% (Figure 19-B). Cell purification procedures were conducted at $4^{\circ} \mathrm{C}$ to minimize in vitro-induced changes in gene expression.

RNA samples extracted from the different groups of samples were labelled and hybridised to the Affymetrix GeneChip ${ }^{\circledR}$ Mouse Gene 2.0 ST array. 


\section{$\mathrm{B} 6 \rightarrow 129$ model}

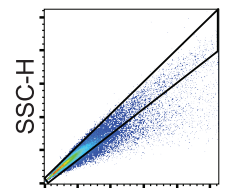

SSC-A

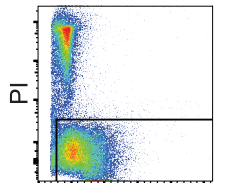

FSC-A

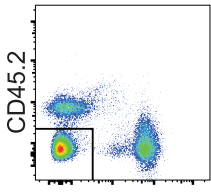

CD45.1

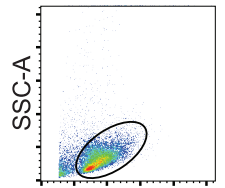

FSC-A

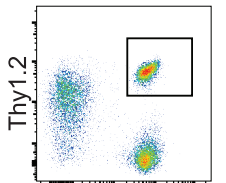

CD45.1

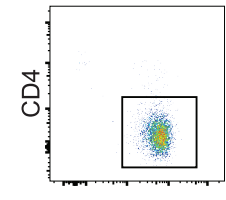

CD8

\section{MataHari T cell model}

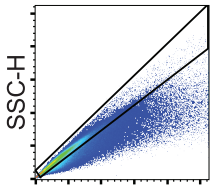

SSC-A

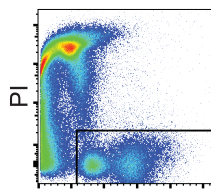

FSC-A

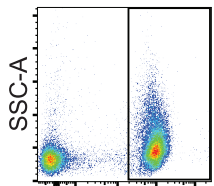

CD45.2

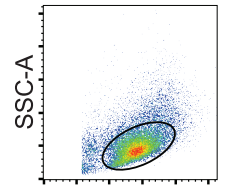

FSC-A

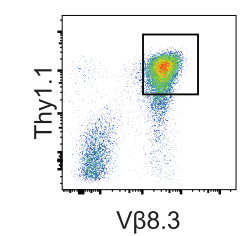

V 88.3

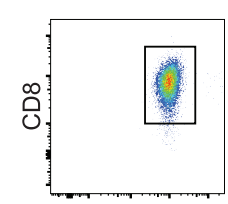

CD45. 2

Figure 17. Strategy for high-purity FACS sorting of donor $\mathrm{CD} 8^{+} \mathrm{T}$ cells. Gating sequence used to select donor derived $\mathrm{CD}^{+} \mathrm{T}$ cells in each GVHD model: exclusion of doublets $\rightarrow$ exclusion of propidium iodide positive cells $\rightarrow$ exclusion of stroma cells $\rightarrow$ morphologic lymphocyte selection $\rightarrow$ selection of donor $\mathrm{CD}^{+}{ }^{+}$cells based on the expression of congenic markers $\rightarrow$ confirmation of CD8 positivity.

A

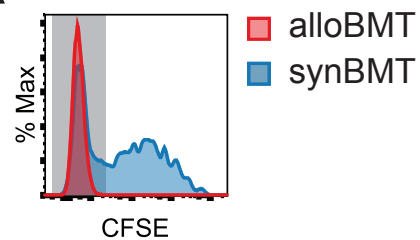

B

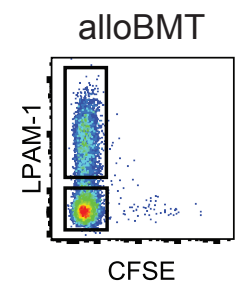

synBMT

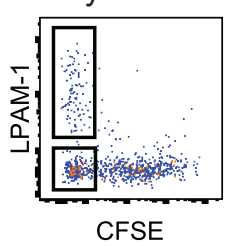

Figure 18. Staining strategy to isolate cells with equivalent number of divisions (A) and homing imprinting (B). (A) To ensure the comparability between groups, CSFE labelling of the $\mathrm{CD}^{+}{ }^{+} \mathrm{T}$ cells prior to infusion into the recipients was used as a means to identify cells that had undergone similar number of divisions. The plot is a representative example of the gating strategy followed to exclusively select cells that had diluted the dye (grey area). CD $8^{+} \mathrm{T}$ cells collected from SLO of synBMT recipients displayed a bimodal staining (blue curve) in contrast to those isolated from alloBMT recipients (red curve) which were uniformly CFSE negative by day 7 post transplant. (B) Donor $\mathrm{CD}^{+} \mathrm{T}$ cells from the blood were subdivided into CFSE ${ }^{\text {low }}$ LPAM- $1^{\text {high }}$ (cells imprinted for gut homing) and CFSE ${ }^{\text {low }}$ LPAM- $1^{\text {low }}$ cells. 
A

B6 $\rightarrow 129$ model

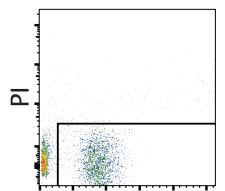

FSC-A

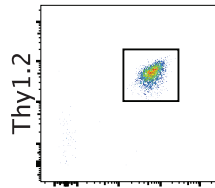

CD45. 1

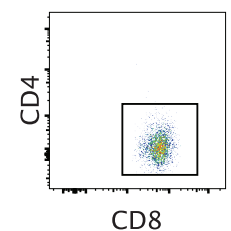

\section{MataHari T cell model}
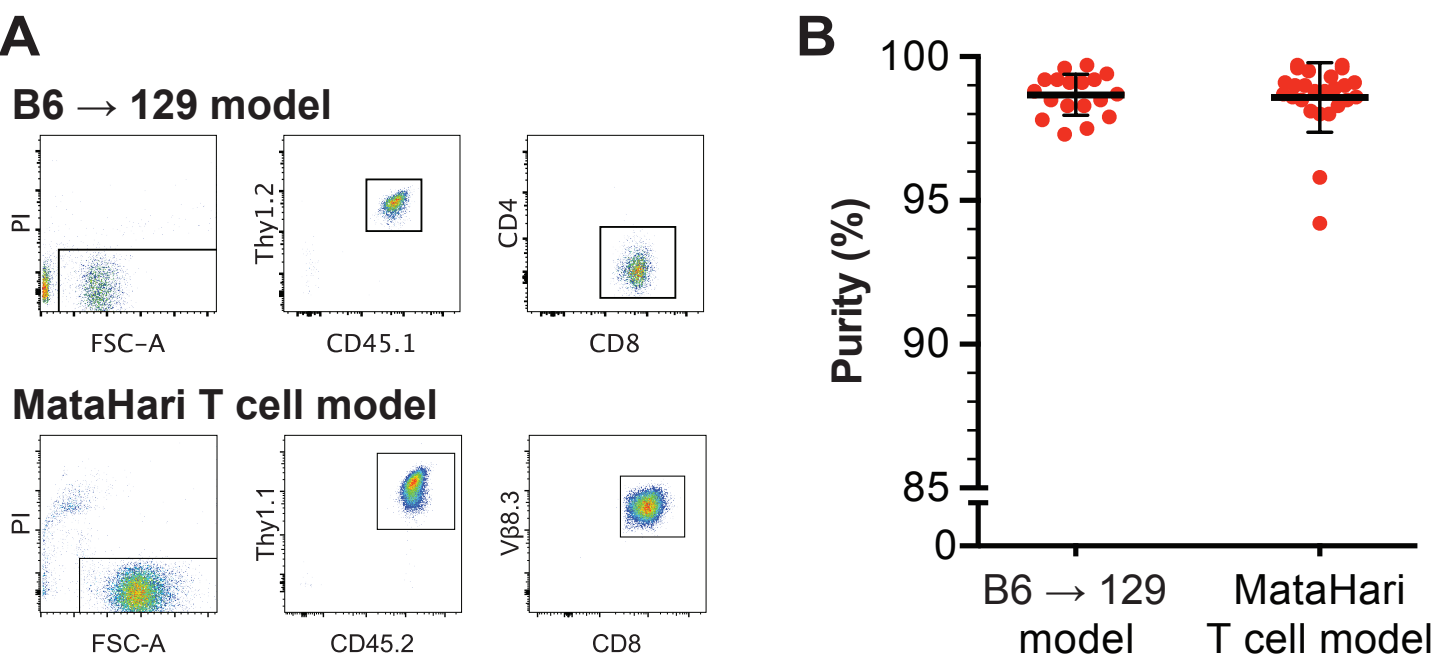

Figure 19. Sort quality check and evaluation of sample purity. (A) Gating strategy used to determine the purity of the isolated populations (viable cells, positive for CD8 and for the donor congenic markers). (B) Purity of the isolated populations (percentage of viable $\mathrm{CD} 8^{+}$cells bearing the donor congenic markers) selected for the RNA extraction used in the microarray study (B6 $\rightarrow 129$ model: $n=18$; MataHari T cell model: $n=45$ ). 


\subsection{Exploratory data analysis}

Unsupervised data analyses consist of exploratory techniques that do not require the incorporation of any prior knowledge and aim to identify groups with similar behaviours (i.e. expression profiles). ${ }^{(271)}$ Some commonly used mathematical tools that characterise the structure of the data include principal component analysis (PCA) and multidimensional scaling (MDS) for dimensionality reduction, ${ }^{(272,273)}$ as well as hierarchical clustering for relationship discovery. ${ }^{(274,275)}$

The advantage of performing PCA on microarray data sets is that it allows visual assessment of the similarities and differences between samples in $2 \mathrm{D}$ or $3 \mathrm{D}$ plots, by virtue of reducing the dimensionality of the data while retaining most of its variation. ${ }^{(276)}$ This reduction is accomplished through the identification of the directions (principal components) along which the variation in the data is maximal, so that each sample can be represented by relatively few values instead of by thousands of variables. ${ }^{(276)}$

\section{a) $B 6 \rightarrow 129$ model}

The two principle components of the gene expression profiles of the $B 6 \rightarrow 129$ data sets are ploted in Figure 20 . The samples have been colour coded to reflect the three study groups (samples from healthy donors in light blue, syngeneic BMT recipient controls in dark blue and allogeneic BMT study subjects in red). Analysis of the bi-plots showed a greater diversity of gene 
expression profiles in the alloBMT setting when compared to the syngeneic BMT controls or the donor samples, consistent with the underlying major differences in cellular developmental stage: donor - naïve; synBMT - antigeninexperienced T cells undergoing homeostasis-driven proliferation; alloBMT antigen-experienced effector T cells.

Clustering algorithms were used to establish groups that share similar characteristics. The result of these techniques can be depicted as dendrograms, tree like structures in which the nodes represent subsets of an expression data set joined to form groups according to their distance or similarity. ${ }^{(227,277)}$ As illustrated in Figure 21, hierarchical clustering of the samples using Pearson's correlation as the dissimilarity distance metric with an average-linkage clustering method confirmed the existence of a great dissimilarity between the gene expression profiles of the alloBMT samples and the other groups. Moreover, within the alloBMT groups, a dichotomy between SLO and the GVHD target-organs was revealed. Evaluation of the validity and stability of the clusters using non-parametric bootstrap methodology ${ }^{(249,278)}$ supported these observations.

In the interest of resolving the similarities and dissimilarities within the alloBMT group and visualise the interrelationships between samples, multidimensional scaling was performed, as it conveys a superior fidelity in the representation of the distances between different cases, particularly for highdimensional geometric objects. ${ }^{(279,280)}$ From Figure 22, it is clear the presence of a rift between the gene expression of T cells in the SLO and in the GVHD target organs. 


\section{b) MataHari T cell model}

To evaluate if the diversity in transcriptional profiles observed in the $B 6 \rightarrow 129$ model could be due to a segregation of subpopulations of $T$ cells according to their TCR repertoire, merely reflecting an asymmetric tissue antigen distribution, the same analysis was performed in the MataHari T cell model. If differences in antigen distribution were the main driver of the dissimilarities in gene expression among donor T cells isolated from different organs, it would be expected that in the MataHari T cell model the diversity in transcriptional profiles would be greatly reduced, as the antigen recognised by the MataHari T cells is uniformly expressed in all tissues.

The results, however, do not support this hypothesis. Both the hierarchical clustering (Figure 23) and the multidimensional scaling analysis (Figure 24) show a similar pattern to that observed in the $B 6 \rightarrow 129$ model. Although, in this model, the donor $\mathrm{CD} 8^{+} \mathrm{T}$ cell population is monoclonal, there still exists a clear difference between the transcription profiles of cells isolated from the SLO and the GVHD target organs. Interestingly, when assessing the variability within these two compartments, it appears that in the $B 6 \rightarrow 129$ model the individual tissue clusters are more discrete than in the MataHari T cell model, suggesting that, even though TCR repertoire variety is not required for the establishment of a SLO - GVHD target organ dichotomy, it could contribute to enhance the differences between tissues.

To further explore this concept, a direct comparison between the 2 data sets was performed, after correction for batch effects with the 'ComBat' function 
of the 'sva' package for ${ }^{(245)}$ Figure 25 depicts the average linkage agglomerative hierarchical clustering of all samples, with the underlying similarity matrix showing the individual Pearson's correlation coefficients colour coded for easier interpretation. As can be observed, the separation between the three groups (donor + synBMT vs alloBMT - SLO vs alloBMT - GVHD target organs) is maintained, and the clustering of the samples appears to reflect the source tissue, rather than the GVHD model. This result validates the previous observations and confirms that the transcriptional diversity observed was largely independent of the TCR repertoire and antigen distribution. 


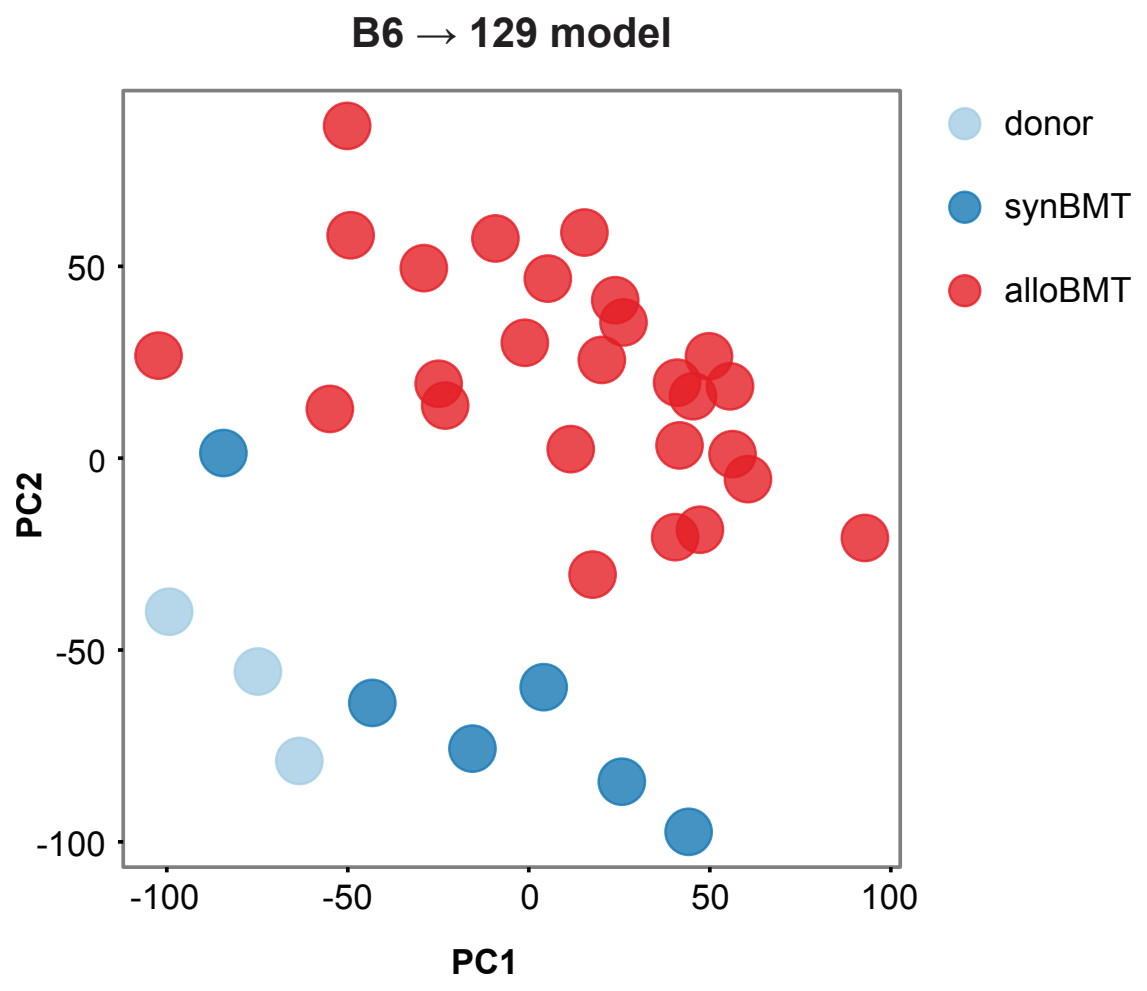

Figure 20. Principal components analysis of the $B 6 \rightarrow 129$ data set. The plot shows the $2 D$ projection of the first two principal components; samples were colour coded according to study groups (light blue: donors; dark blue: syngeneic BMT; red: allogeneic BMT). It is noteworthy a greater dispersion of the points in the alloBMT group when compared to the syngeneic BMT controls or the donor samples. $\mathrm{PC} 1,1^{\text {st }}$ principal component; $\mathrm{PC} 2,2^{\text {nd }}$ principal component. 


\section{B6 $\rightarrow 129$ model}

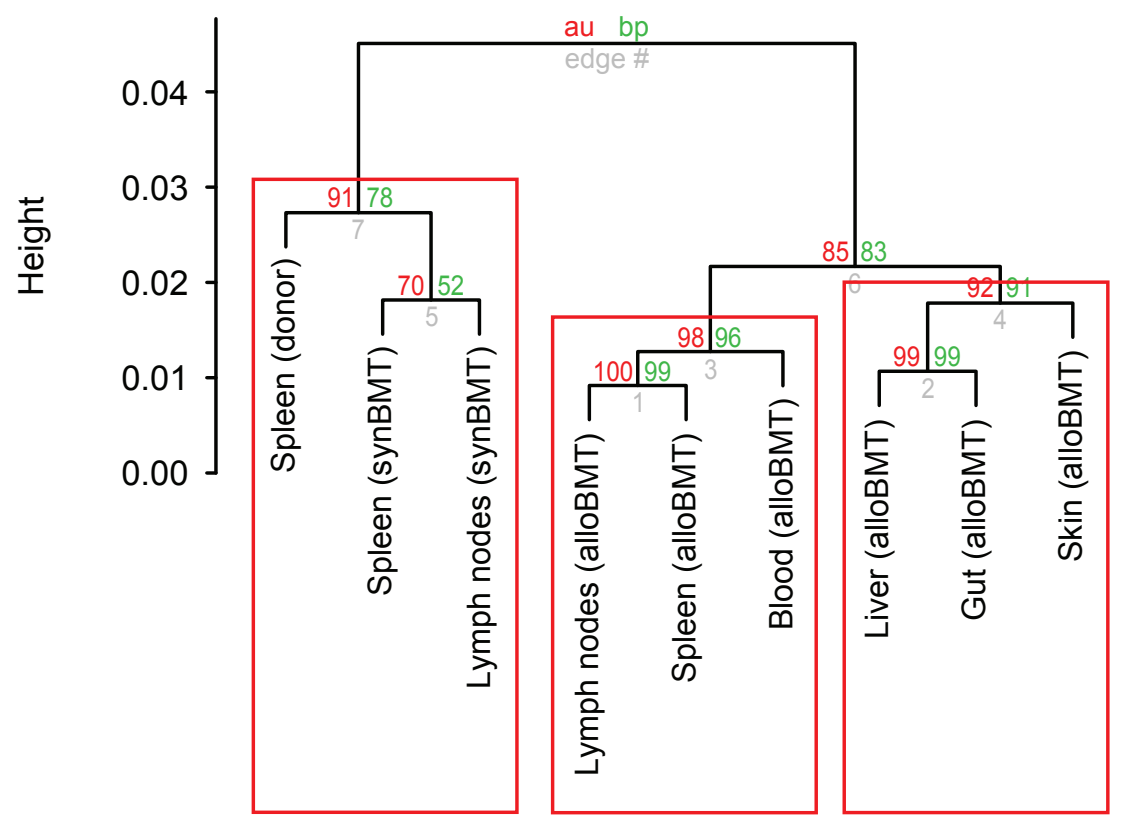

Distance: correlation Cluster method: average

Figure 21. Hierarchical clustering of the $B 6 \rightarrow 129$ data set, validated through non-parametric bootstrap methodology. Groups represent the average expression of the biological replicates. Three clusters are identified (red boxes), separating the donor + synBMT, from the alloBMT secondary lymphoid organs (SLO), from the alloBMT GVHD target organs. The approximate unbiased p-values ( $\mathrm{AU}$, in red) and the bootstrap probability (BP, in green) are shown above each node (identified by the grey numbers). 


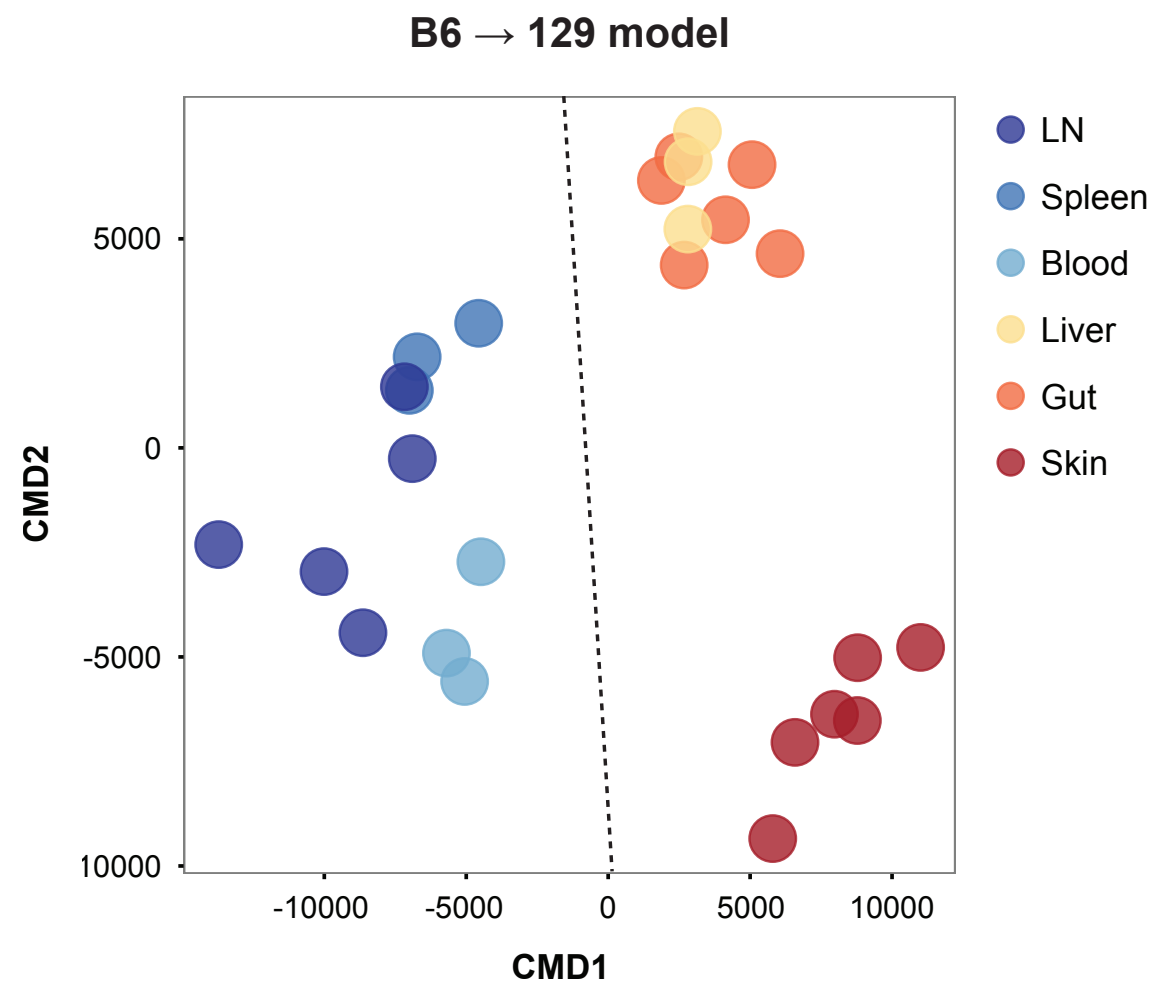

Figure 22. Multidimensional scaling analysis of the alloBMT samples from the $B 6 \rightarrow 129$ data set. A rift between the SLO and the GVHD target organs is apparent. CMD1, $1^{\text {st }}$ coordinate of multidimensional scaling; CMD2, $2^{\text {nd }}$ coordinate of multidimensional scaling; LN, lymph nodes. 


\section{MataHari T cell model}

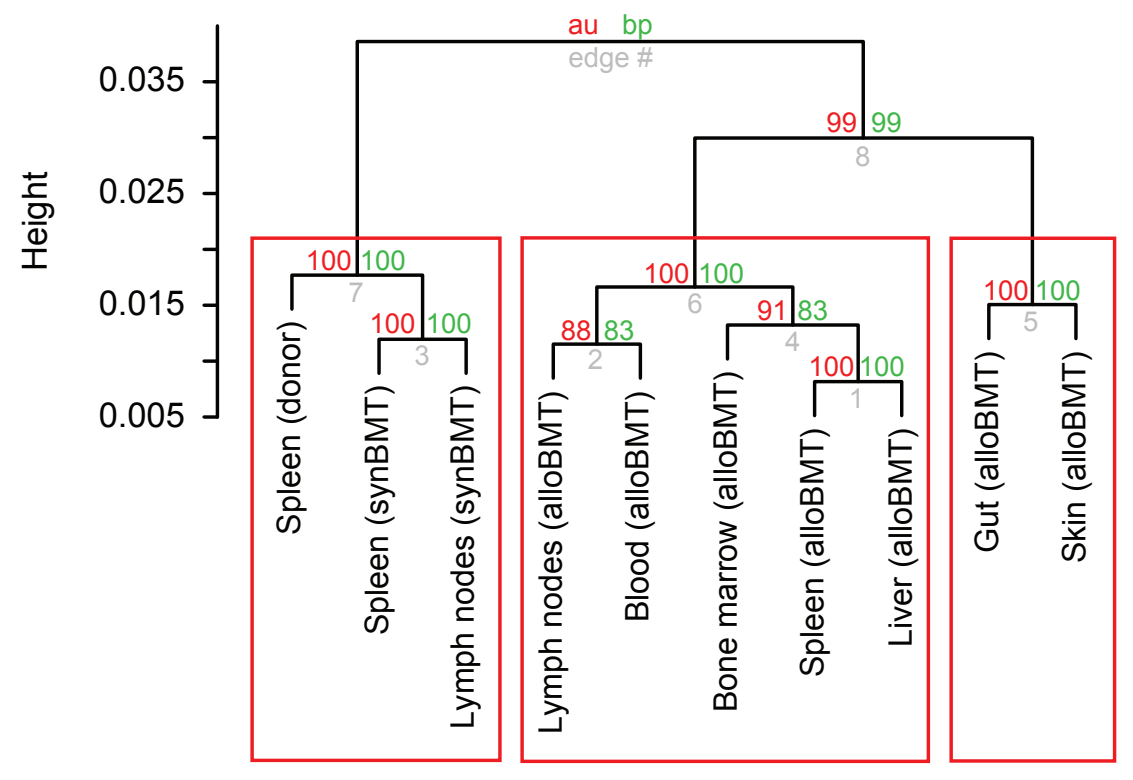

Distance: correlation

Cluster method: average

Figure 23. Hierarchical clustering of the MataHari $T$ cell data set, validated through non-parametric bootstrap methodology. Groups represent the average expression of the biological replicates. Similarly to the $\mathrm{B} 6 \rightarrow 129$ data set, three clusters are identified (red boxes), separating the donor + synBMT, from the alloBMT secondary lymphoid organs (SLO), from the alloBMT GVHD target organs. The approximate unbiased p-values (AU, in red) and the bootstrap probability (BP, in green) are shown above each node (identified by the grey numbers). 


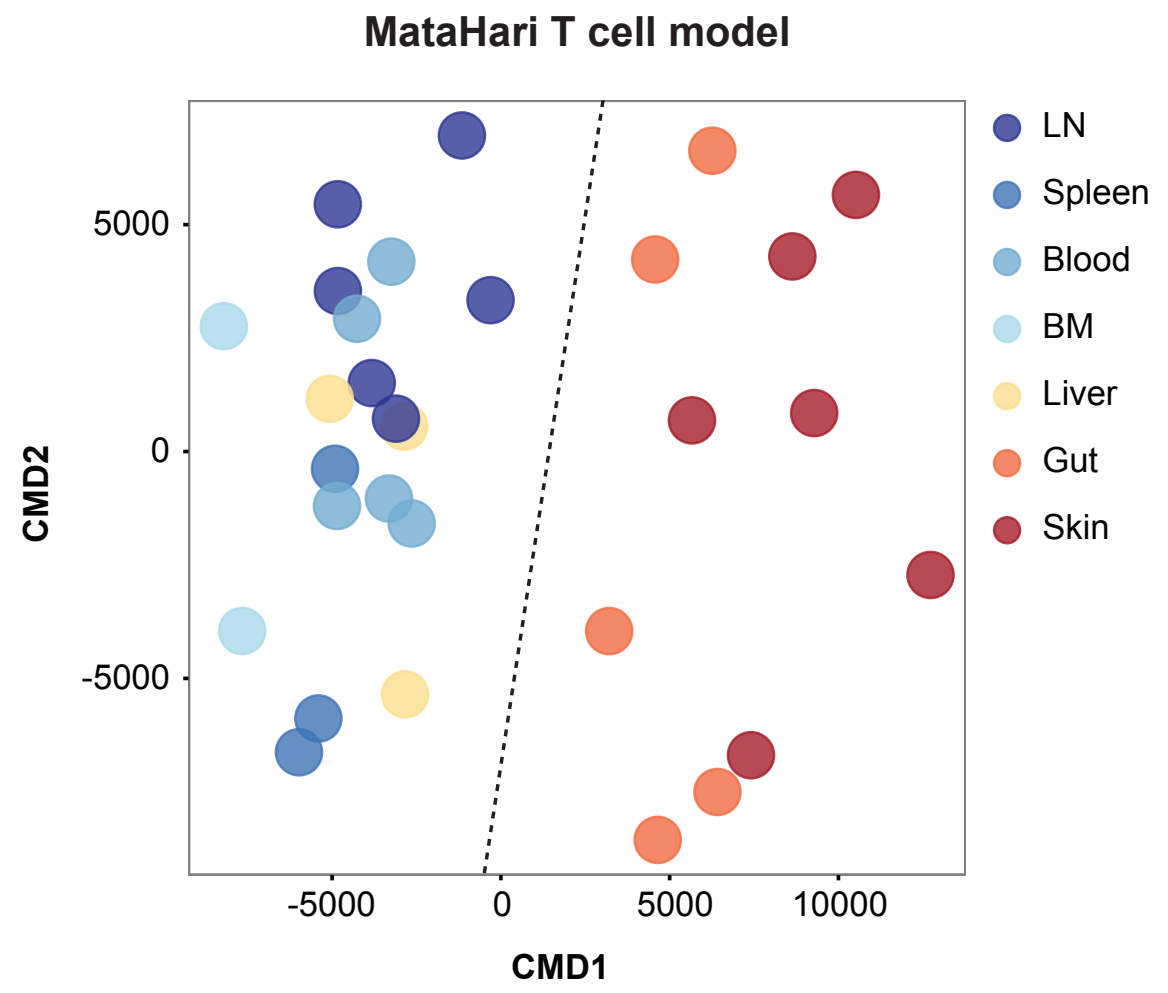

Figure 24. Multidimensional scaling analysis of the alloBMT samples from the MataHari T cell data set. The dichotomi between SLO and GVHD target organs is maintained, however in the MataHari T cell model the difference is less pronounced with the liver samples being closer to the SLO than to the remaining GVHD target organs. CMD1, $1^{\text {st }}$ coordinate of multidimensional scaling; CMD2, $2^{\text {nd }}$ coordinate of multidimensional scaling; LN, lymph nodes. 


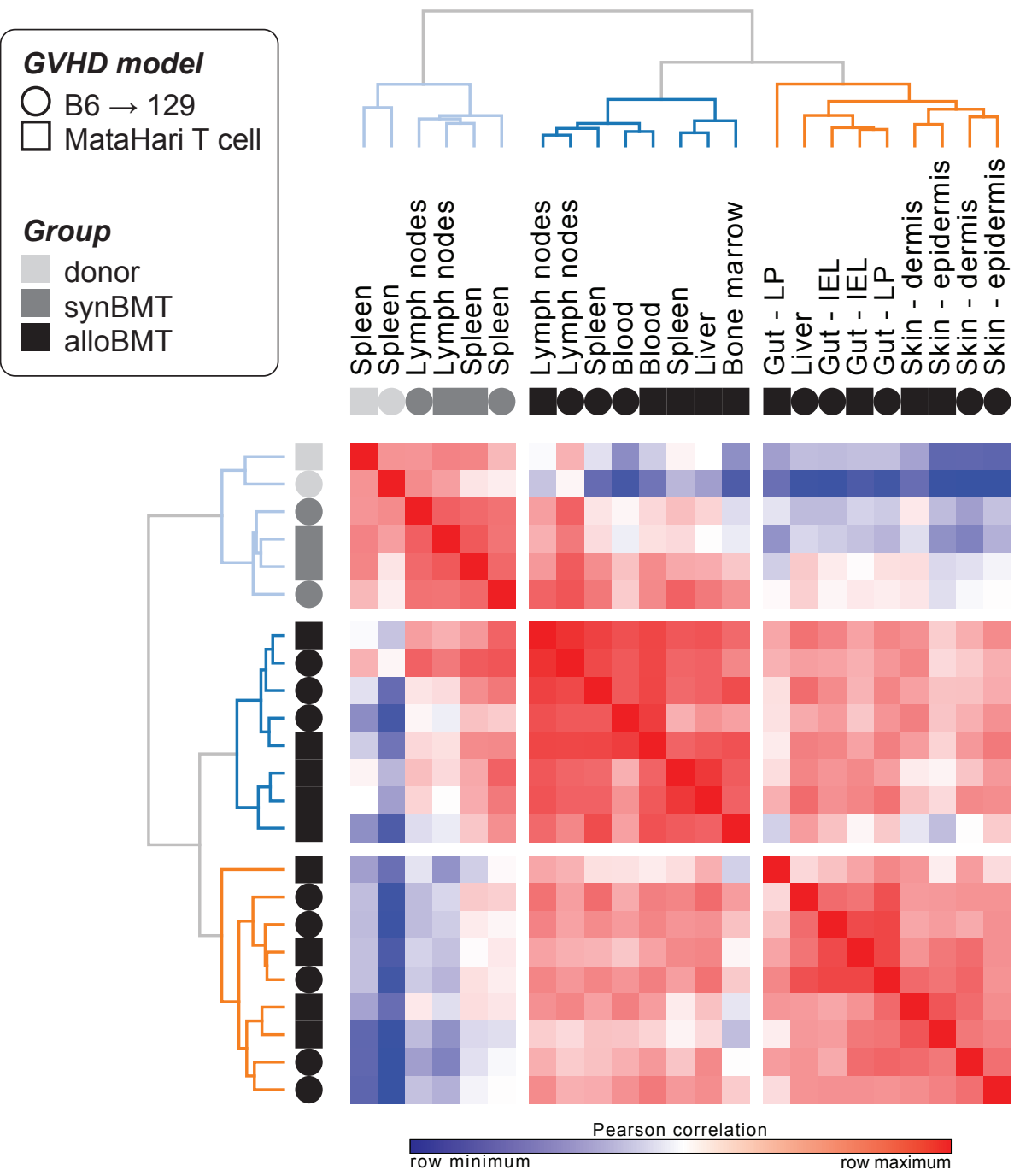

Figure 25. Transcriptional profiles are independent of the TCR repertoire and do not reflect antigen distribution. Average linkage agglomerative hierarchical clustering of all samples from both experimental models and similarity matrix with colour coded Pearson's correlation coefficients shows that sample clustering reflects the study groups (light grey: donor; dark grey: synBMT; black: alloBMT) and the tissues of origin, irrespective of GVHD model (circles: $B 6 \rightarrow 129$; squares: MataHari T cell). 


\subsection{SLO - GVHD target organ dichotomy reflects disparities in differentiation status of donor $T_{\text {eff }}$ cells}

The magnitude of the divergence in gene expression between the SLO and the GVHD target organs was further evaluated in both models by performing gene set enrichment analysis (GSEA) comparing the transcriptional profiles of these two groups. GSEA is a method that identifies biologically meaningful, even if subtle, differences in gene expression, through the analysis of coordinated changes in sets of functionally related genes. ${ }^{(254)}$

Figure 26 summarises the results from GSEA comparing the GVHD target organs to the SLO in the B6 $\rightarrow 129$ model, using the the C5:BP (Gene Ontology - Biological Process) collection from the Molecular Signatures Database (MSigDB). The Gene Ontology (GO) terms enriched in the GVHD target organs were organised into an enrichment map using Cytoscape "Enrichment Map" plug-in. ${ }^{(281)}$ This network representation of the data clusters together mutually overlapping gene-sets, where the node size represents the number of genes in the gene-set; edge thickness is proportional to the overlap between gene-sets; and the enrichment p-value is mapped to the node colour as a white-to-red gradient. Analysis of the GO enrichment map reveals that the genes differentially expressed in the GVHD target organs in comparison to the SLO are involved mainly in processes of transcription and protein metabolism; signal transduction; cell component transport and localisation; regulation of cell 
cycle, proliferation, development and apoptosis; and are linked to the establishment of the immune reaction.

In order to gain more insight into the pathways up-regulated in the GVHD target organs, a pair-wise comparison of the SLO and GVHD target organs was performed using the C2:CP:KEGG collection (Curated canonical pathways KEGG PATHWAY), both on the $\mathrm{B} 6 \rightarrow 129$ and the MataHari $\mathrm{T}$ cell models. A total of 154 pathways were identified as being enriched in the GVHD target organs, with an extensive overlap between the two models: $87.2 \%$ of pathways found in the MataHari T cell model were also present in the $B 6 \rightarrow 129$ model (Figure 27). The top 10 pathways common to both models, listed in Table 12, reflect a clear enrichment for pathways related to the effector mechanisms of adaptive immunity and to alloreactivity.

Moreover, the analysis of the expression profiles of a selection of genes frequently used to characterise the stages of $T$ cell development ${ }^{(282-286)}$ (Figure 28, data shown only for the $\mathrm{B} 6 \rightarrow \mathbf{1 2 9}$ model) revealed a coordinated change in the expression of a panoply of markers associated with $\mathrm{CD}^{+} \mathrm{T}$ cell effector function consistent with the transition from a naïve phenotype in the donor + synBMT group, to a post-TCR engagement activation status in the alloBMT group, which ranged from early to late effector phenotypes in the SLO and GVHD target organs, respectively. A similar trend was observed when the differentiation status of the donor $\mathrm{CD}^{+} \mathrm{T}$ cells in each tissue was evaluated by calculating the enrichment for published genetic signatures associated with Tc1/Tc17 polarisation, ${ }^{(287)}$ pathogenicity, ${ }^{(288)}$ and cytokine or effector response, ${ }^{(285)}$ using single-sample GSEA (Figure 29). Additionally, this 
analysis revealed a wide range of enrichment scores across tissues and a dissonance between the polarisation of the T cells and their cytotoxicity; while the Tc1 and Tc17 transcriptional signatures are upregulated early on in the SLO, the maximum expression of effector molecules associated with tissue injury is only achieved in the GVHD target organs. 

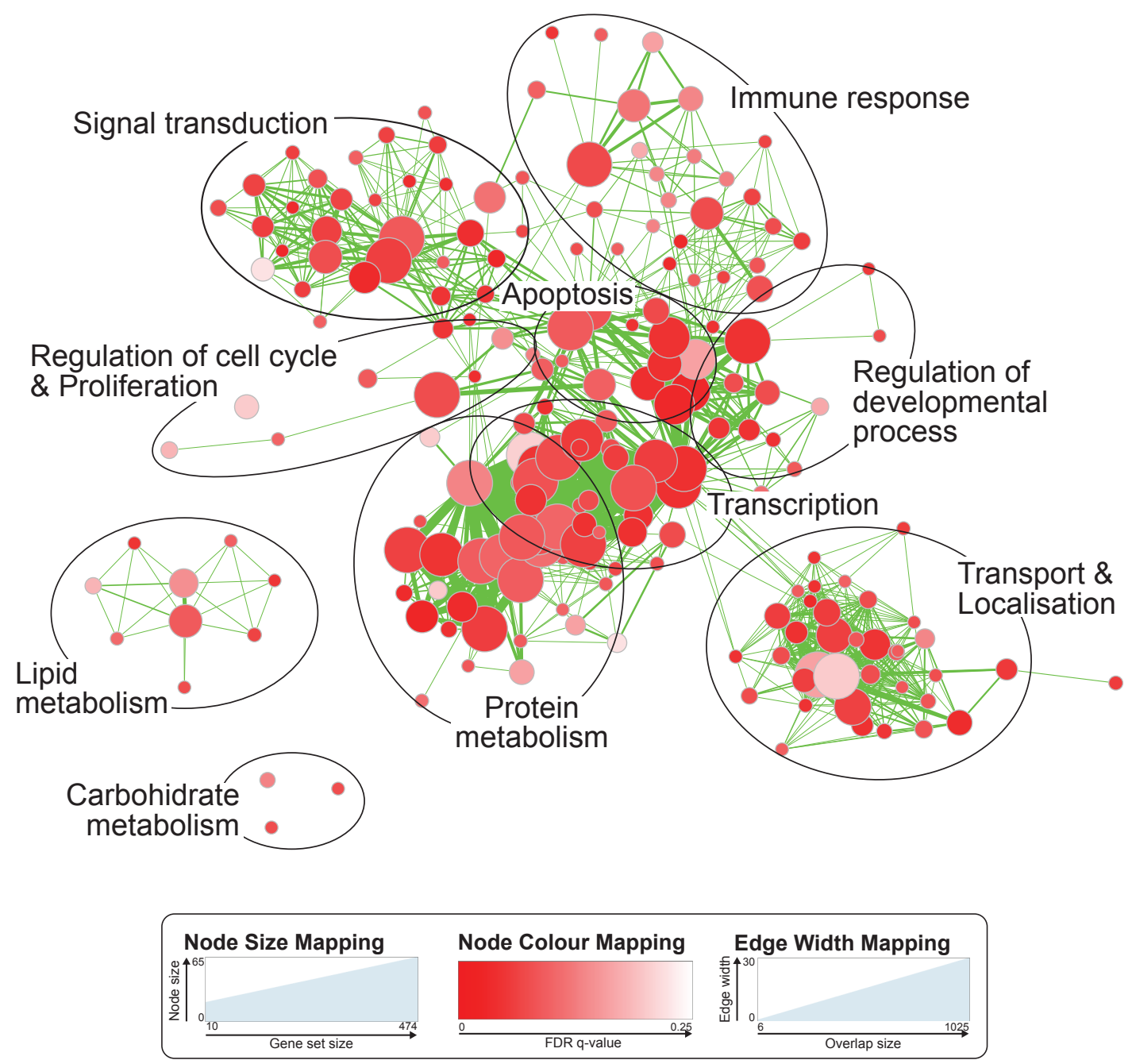

Figure 26. Transcriptional differences between SLO and GVHD target organs are biologically meaningful. Network visualization of GSEA based on the differentially expressed genes in the GVHD target organs vs the SLO, using EnrichmentMap (B6 $\rightarrow 129$ data set). Enriched GO terms are depicted by red nodes, where colour represents the corresponding FDR-adjusted enrichment P-value (q-value), size is proportional to the number of genes in each node, edge thickness indicates overlap of genes between nodes, and the theme of each clusters is specified. 


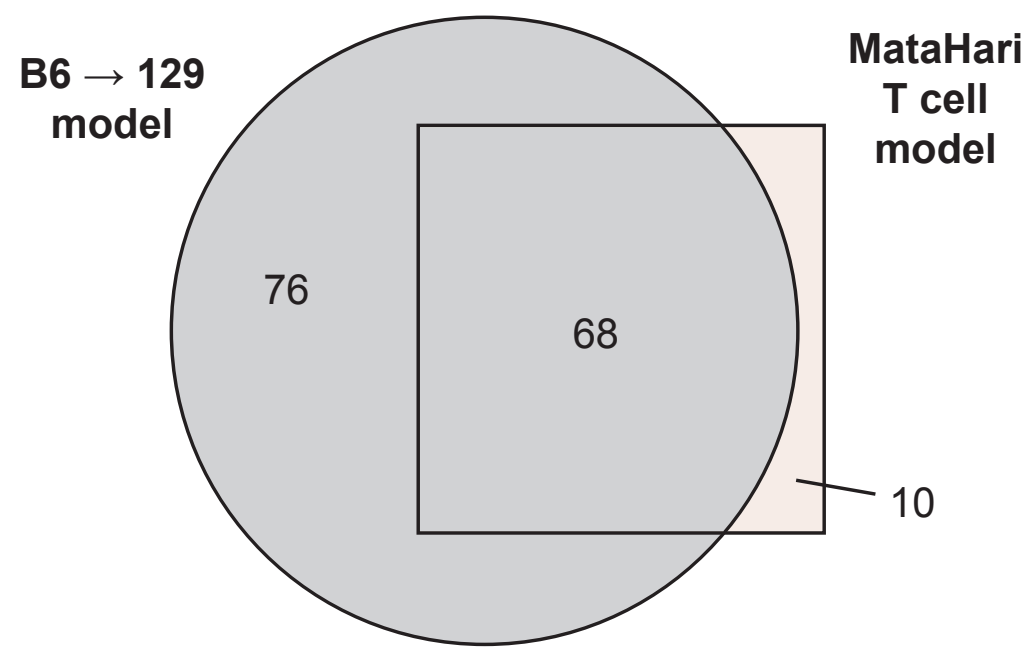

Figure 27. Venn diagram representing the overlap of enriched gene sets from the KEGG PATHWAY collection in the GVHD target organs vs the SLO, found in the B6 $\rightarrow 129$ (circle) and the MataHari T cell (square) models. An extensive overlap between the two models is observed.

Table 12. Top 10 pathways enriched in GVHD target organs vs SLO common to both data sets (MSigDB C2:CP:KEGG collection).

\begin{tabular}{lrcc}
\hline Pathway name & Size & $\begin{array}{c}\text { NOM } \\
\text { p-val }\end{array}$ & $\begin{array}{c}\text { FDR } \\
\text { q-val }\end{array}$ \\
\hline \hline T cell receptor signalling pathway & 104 & $<0.0001$ & 0.0001 \\
Endocytosis & 171 & $<0.0001$ & 0.0001 \\
B cell receptor signalling pathway & 71 & $<0.0001$ & 0.0016 \\
MAPK signalling pathway & 255 & $<0.0001$ & 0.0043 \\
Natural killer cell mediated cytotoxicity & 96 & $<0.0001$ & 0.0066 \\
Chemokine signalling pathway & 162 & $<0.0001$ & 0.0069 \\
Lysosome & 114 & $<0.0001$ & 0.0074 \\
Cytokine-cytokine receptor interaction & 225 & $<0.0001$ & 0.0220 \\
Graft-versus-host disease & 27 & 0.0051 & 0.0223 \\
Allograft rejection & 29 & 0.0056 & 0.0224 \\
\hline
\end{tabular}



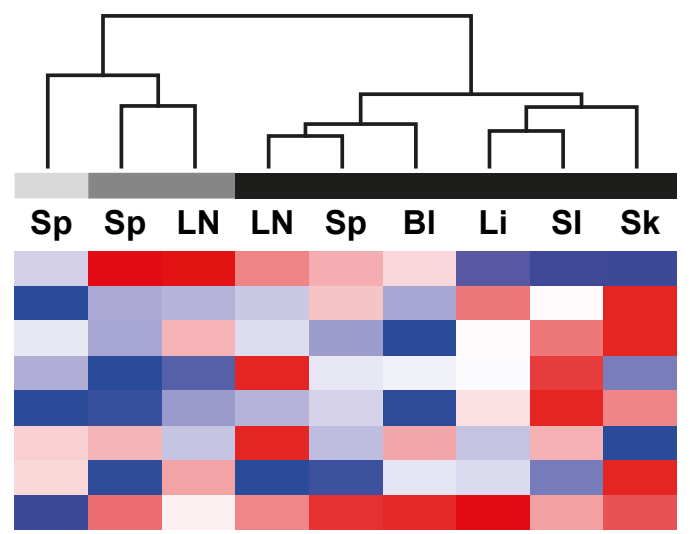

EOMES

IRF4

RELB

RORC

STAT3

STAT4

STAT5A

TBX21

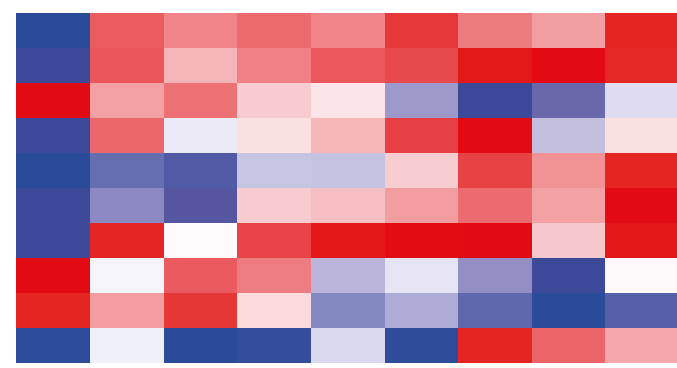

CCR2

CCR5

CCR7

CXCR3

CXCR6

IL2RA

IL2RB

IL7R

JAK1

JAK2

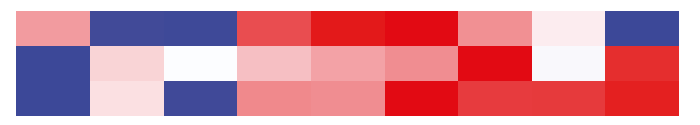

ITGA4

ITGAL

ITGAX

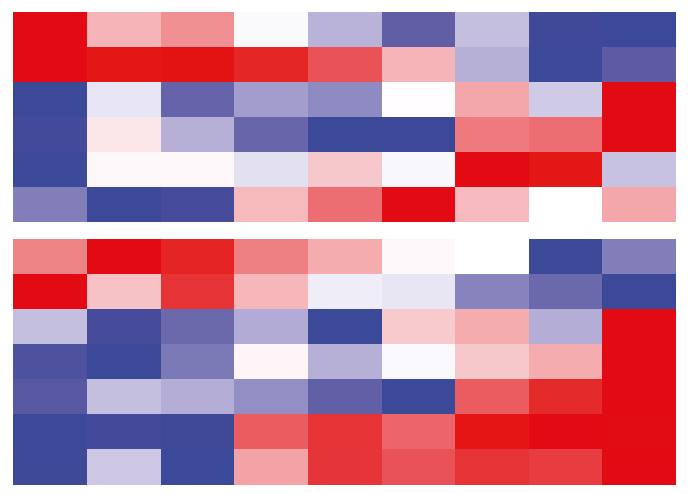

PECAM1

SELL

CD44

CD69

CD160

SPN

BTLA

CD27

CD28

ICOS

CD274

PDCD1

HAVCR2

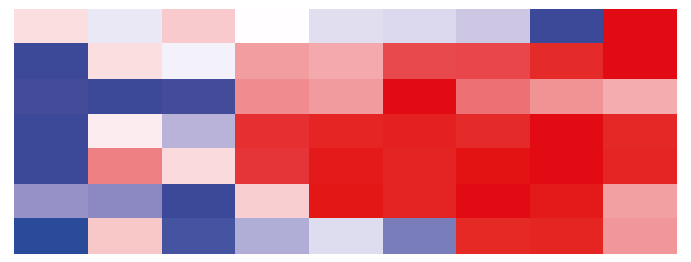

FAS

FASLG

KLRG1

GZMA

GZMB

PRF1

TNFSF10

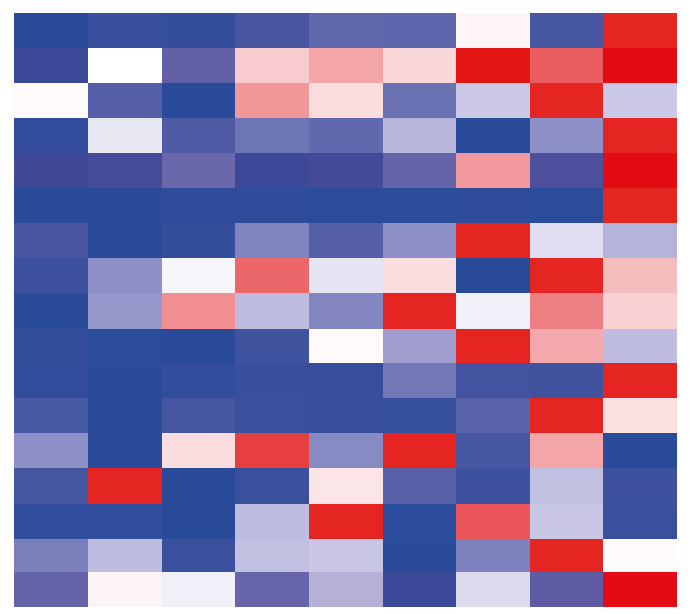

CSF2

IFNG

IL1A

IL1B

IL2

IL3

IL4

IL7

IL9

IL10

IL13

IL17A

IL17F

IL18

IL21

IL22

TNF

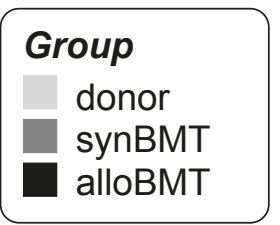

Z-score of expression row min row max
Figure 28. Heat map of selected genes commonly used to characterise T cell differentiation, including: transcription factors, chemokine/cytokine receptors, signalling molecules, integrins, activation markers, coregulatory molecules, and effector function molecules. Note the shift from a naïve phenotype in the donor + synBMT group, to an early activated status in the alloBMT SLO and a late effector phenotype in the GVHD target organs. Data from the $\mathrm{B} 6 \rightarrow 129$ model. Sp, spleen; LN, lymph nodes; $\mathrm{Bl}$, blood; $\mathrm{Li}$, liver; SI, small intestine; Sk, skin. 


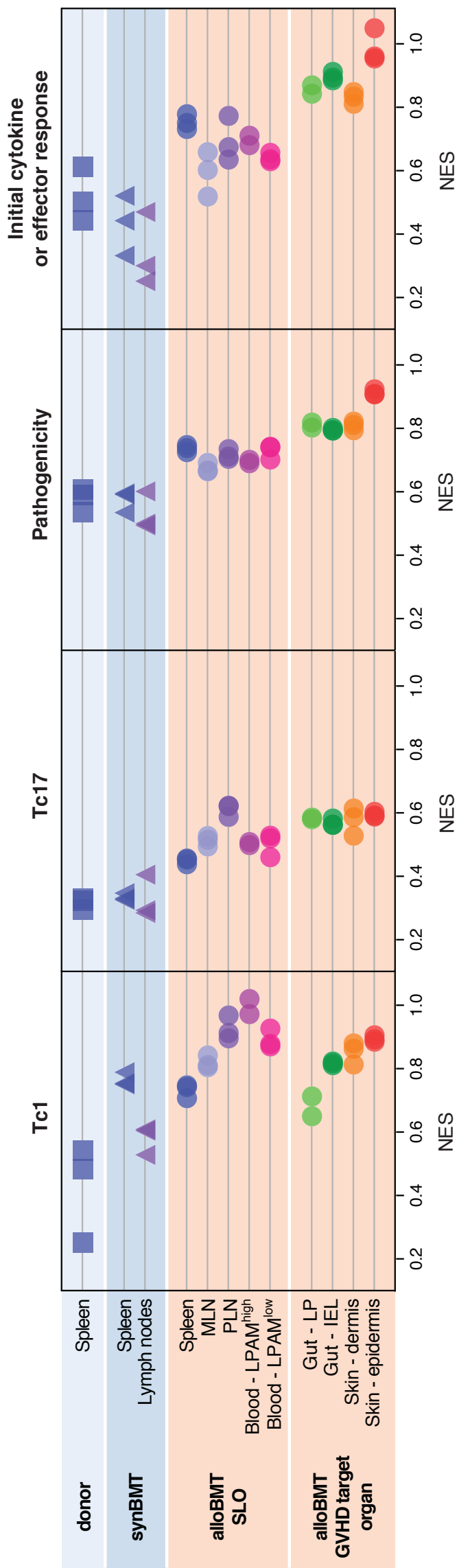

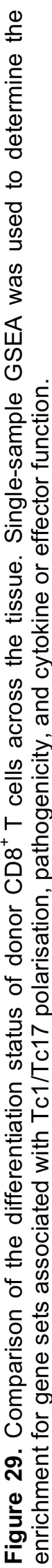




\subsection{Transcriptome diversity is neither due to early trafficking of effectors to the peripheral tissues nor to differential homing imprinting of donor-derived $\mathrm{T}$ cells}

One possible explanation for the differences in transcriptional profiles described could be that at the time point the samples were collected the effector cells have already migrated out of the SLO towards the peripheral tissues, and those that remain in the $L N$ and spleen represent the subpopulations of $T$ cells that were either ineffectively primed or that are still going through the process of activation.

To test this hypothesis, a second set of experiments were performed using the MataHari model in which the HSCT recipients were treated with FTY720 (a sphingosine-1-phosphate antagonist) to trap T cells in the SLO (Figure 30). By starting the treatment at D+3 post-transplant (a time point before MataHari T cells are easily detectable in blood or GVHD organs) the lymphocyte egress from the lymph nodes was effectively blocked, as demonstrated by the marked reduction in circulating donor $\mathrm{CD}^{+} \mathrm{T}_{\text {eff }}$ cells in FTY720 treated animals in comparison to the controls (Figure 31).

Analysis of the transcriptional profiles of donor $\mathrm{CD}^{+} \mathrm{T}_{\text {eff }}$ cells isolated from the lymph nodes prior and post-treatment revealed that at $D+3$ donor $T$ cells were in fact closer to the profiles of naïve input T cells, while at $D+7$ there was no significant difference between FTY720-treated subjects and 
PBS-treated controls (Figure 32). The data therefore refute the hypothesis that the differences in gene expression between $\mathrm{T}_{\text {eff }}$ cells isolated from SLO or GVHD target organs can be due to early egress of optimally activated $T_{\text {eff }}$ cells.

It has been demonstrated that tissue tropism imprinting is required for the induction and maintenance of acute GVHD. ${ }^{(289,290)}$ As such, the averaging of gene expression across several tissues could potentially conceal the convergence of transcriptional programs of $\mathrm{T}_{\text {eff }}$ cells from anatomically related SLO and GVHD target organs. To investigate this, LN and blood samples from the MataHari T cell data set were subdivided into their two major constituents (mesenteric and peripheral LN; LPAM-1 ${ }^{\text {high }}$ and LPAM-1 ${ }^{\text {low }}$ blood T cells) and the transcriptional profiles of $\mathrm{T}_{\text {eff }}$ cells derived from gut and skin, their respective draining LN, and GVHD target organ-imprinted peripheral blood subsets were compared. As depicted in Figure 33, the segregation SLO and GVHD target organ $T_{\text {eff }}$ cell profiles was maintained, with no evidence that local imprinting was linked to greater skin or gut-like effector differentiation. 


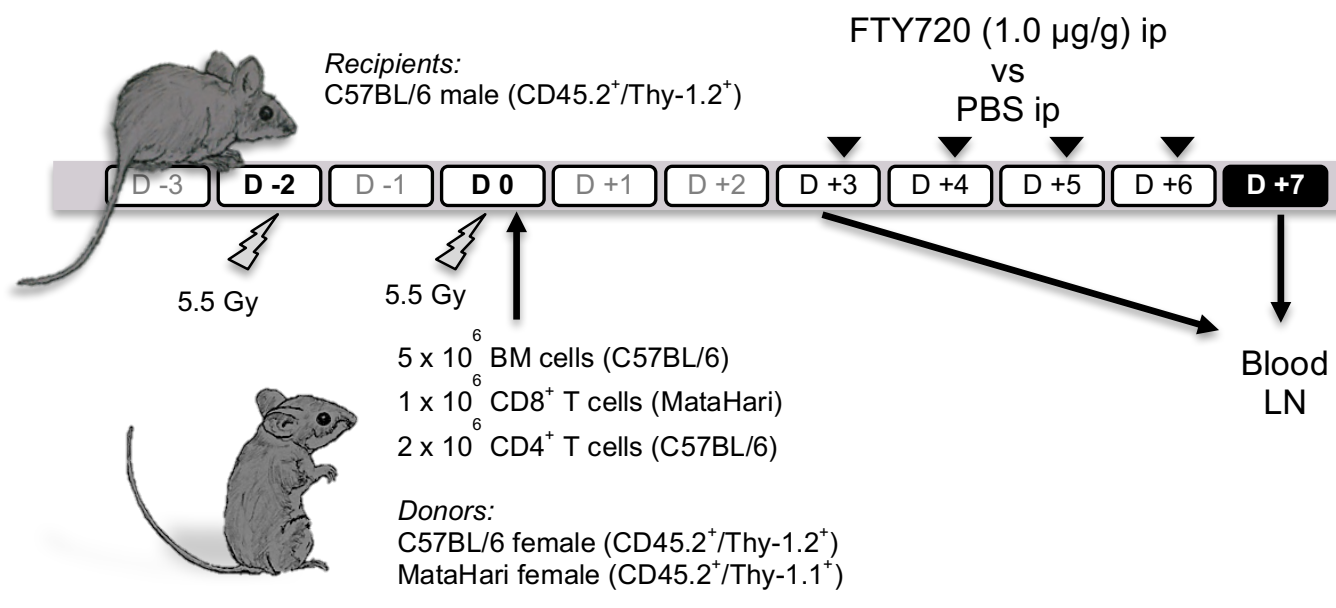

Figure 30. Experimental design to evaluate the effect of SLO lymphocyte egress blockage on the transcriptional profile of donor $\mathrm{CD}^{+} \mathrm{T}_{\text {eff }}$ cells in the lymph nodes. $\mathrm{BM}$, bone marrow; LN, lymph nodes. 

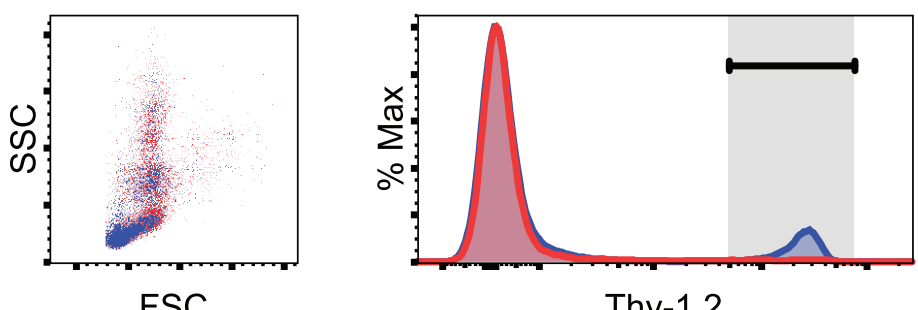

FTY720 2.3\%

PBS $\quad 11.1 \%$

Thy-1.2

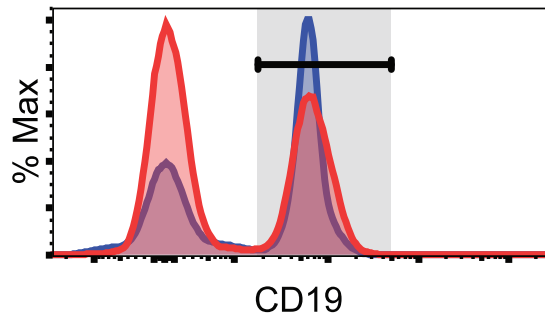

FTY720 42\%

PBS $\quad 59 \%$

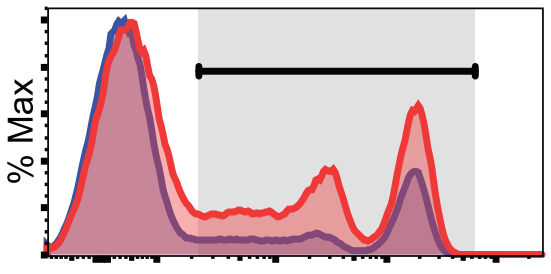

FTY720 $18.1 \%$

PBS $\quad 14.2 \%$

Ly6G (Gr1)

FTY720

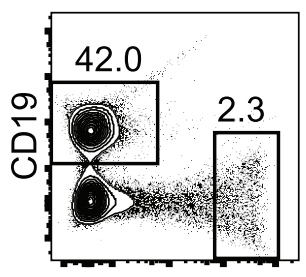

Thy-1.2
PBS

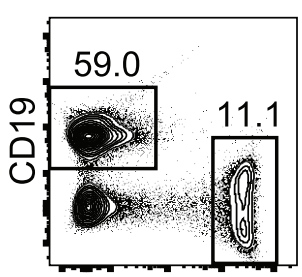

Thy -1.2

Figure 31. Treatment with FTY720 blocks lymphocyte egress from the SLO. Blood samples from FTY720- and PBS-treated animals were collected at D+7 post-transplant. A marked decrease in circulating lymphocytes is observed, affecting particularly T cells ( 4.8 fold reduction in Thy $-1.2^{+}$cells vs 1.4 fold reduction in $\mathrm{CD} 19^{+}$cells), while neutrophil (Ly6G-Gr1 ${ }^{+}$cells) counts remain fairly unaffected. 


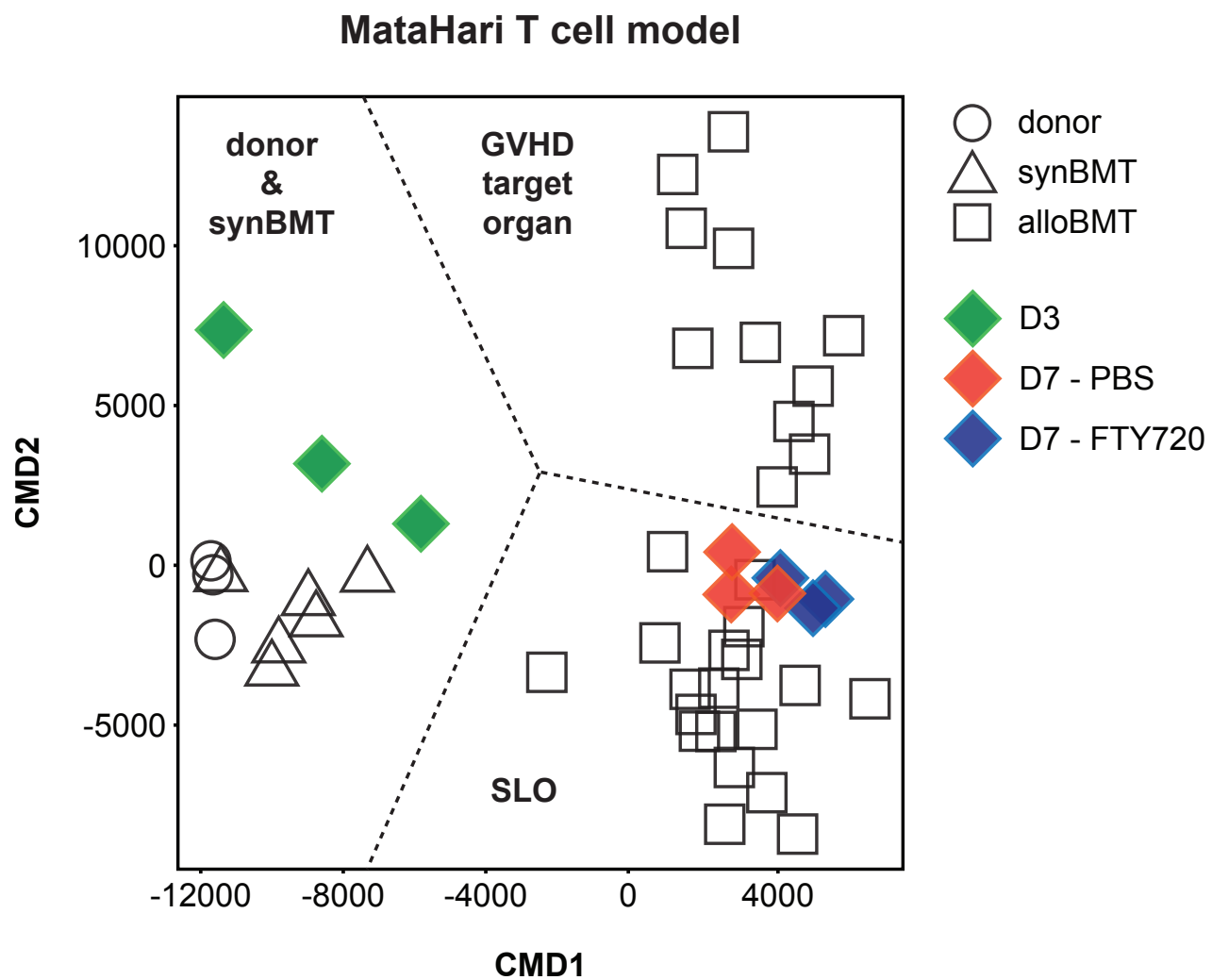

Figure 32. Differences in gene expression between $T_{\text {eff }}$ cells are not due to early egress of optimally activated $T_{\text {eff }}$ cells. Multidimensional scaling analysis of the transcriptional profiles of donor $\mathrm{CD}^{+} \mathrm{T}$ cells isolated from the $\mathrm{LN}$ at $\mathrm{D}+3$ and $\mathrm{D}+7$ post-transplant in animals treated with FTY720 or PBS showing that (1) at D+3 donor T cells in the lymph nodes are very similar to those from the donor and syngeneic BMT controls; (2) at D+7 there are no significant differences between FTY720- and PBS-treated samples. The data suggests that trafficking of effector cells to the periphery does not determine the differences observed between SLO and GVHD target organs. CMD1, $1^{\text {st }}$ coordinate of multidimensional scaling; CMD2, $2^{\text {nd }}$ coordinate of multidimensional scaling. 


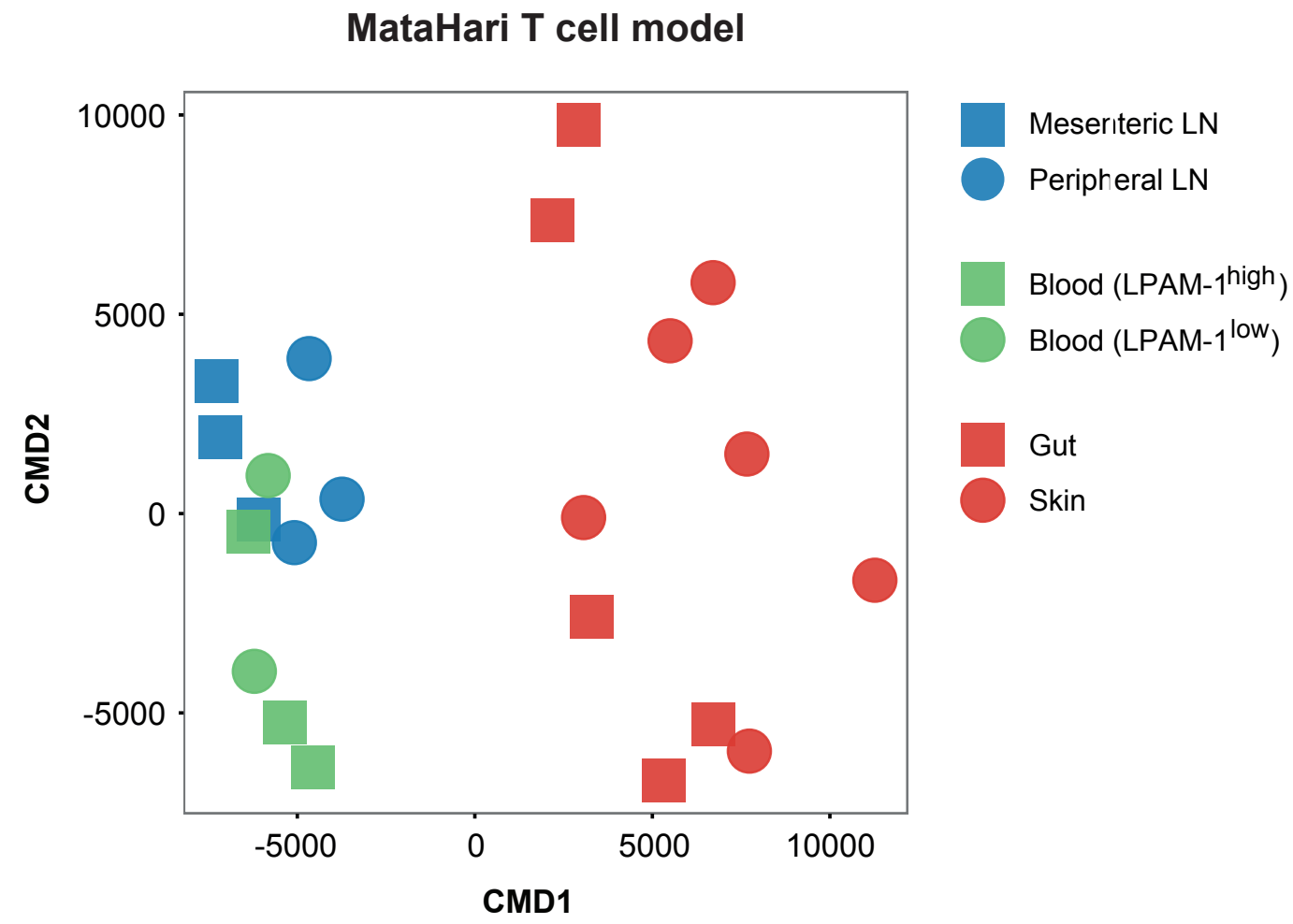

Figure 33. Diversity of transcription profiles is not explained by differences in homing imprinting of donor T cells. Multidimensional scaling analysis of the transcriptional profiles of donor $\mathrm{CD}^{+}$ $T_{\text {eff }}$ cells isolated from the gut and skin, their respective draining $L N$, and GVHD target organ-tropic peripheral blood subsets showed no preferential clustering of samples from anatomically related sources (mesenteric LN / LPAM- $1^{\text {high }}$ blood subset / gut vs peripheral LN / LPAM-1 ${ }^{\text {low }} /$ skin). LN, lymph nodes; CMD1, $1^{\text {st }}$ coordinate of multidimensional scaling; CMD2, $2^{\text {nd }}$ coordinate of multidimensional scaling. 


\subsection{Discussion}

In this project two murine models of miHA-mismatch HSCT were chosen to simulate the most frequent type of HSCT in the clinic, the HLA-matched HSCT $(\mathrm{B} 6 \rightarrow 129$ model), and the common $\mathrm{F} \rightarrow \mathrm{M}$ miHA mismatch associated with increased risk of GVHD development (MataHari T cell model). The first stage of animal model based translational research involves the validation of the experimental models used to characterise the incidence, severity and pathologic evidence of GVHD. Characterisation of the $\mathrm{B} 6 \rightarrow 129$ model was performed in the beginning of the project in collaboration with $\mathrm{Dr}$ Thomas Conlan who also characterised the MataHari $\mathrm{T}$ cell model for his own PhD project. ${ }^{(217)}$ Both models were shown to reproduce the clinical features of acute GVHD, with similar kinetics and severity (Figure 11 and Figure 15), and with the same pattern of organ involvement (Figure 12 and Figure 16).

Previous studies have identified $\mathrm{H} 60$ as a dominant miHA in the $\mathrm{B} 6 \rightarrow 129$ combination, inducing expansion of $\mathrm{H} 60$-specifc $\mathrm{CD}^{+} \mathrm{T}$ cell clones that can amount up to $\sim 20 \%$ of the total host $C D 8^{+}$T cell population by $D+10$ post-HSCT. ${ }^{(291)}$ During the characterisation of the model, the contribution of the H60-specifc $\mathrm{CD}^{+} \mathrm{T}$ cell subset was evaluated and it was determined that, although it represents a dominant population, at D+6 its frequency is usually less than half of that reported in the literature for $D+10$, and no significant difference was observed among the different organs (Figure 13). It is therefore 
very unlikely that the dominance of the anti-H60 alloreactive response may reduce the diversity in transcription profiles in this model.

Indeed, exploratory analysis of the data set showed a great diversity in transcriptional profiles in the alloBMT group with a clear distinction between the SLO and the GVHD target organs. Moreover, in the GVHD target organs two main clusters were identified, one comprising the liver and the gut samples, the other containing the skin samples (Figure 21 and Figure 22). The same pattern was also observed in the MataHari T cell model, with significant diversity in the transcriptional profiles of donor $\mathrm{T}_{\text {eff }}$ cells derived from the individual organs (Figure 23 and Figure 24).

Remarkably, although there are significant differences between the $\mathrm{B} 6 \rightarrow 129$ and the MataHari $\mathrm{T}$ cell models (in the former the donor $\mathrm{T}$ cell population is polyclonal and respond to a multitude of miHA antigens, while in the latter the donor alloreactive response is mediated by a monoclonal population that recognise a single antigen), direct comparison of the transcriptional profiles of all the samples from both data sets revealed a great degree of correspondence between them (Figure 25). It is noteworthy that the samples clustered according to the experimental groups (donor, synBMT, alloBMT) and organ of origin rather than the experimental model, implying that the differences among tissues are more prominent at driving gene expression diversity than dissimilarities between experimental models. The consistent divergence between SLO and GVHD in both the polyclonal and monoclonal systems further supports the conclusion that this effect is independent of TCR repertoire and antigen distribution. 
An interesting difference between the two models is the behaviour of the samples derived from the liver. While in the $\mathrm{B} 6 \rightarrow 129$ model the liver samples segregate with the gut, in the MataHari T cell model their transcriptional profile appears to be closer to that of the SLO. Although in both models there is an extensive lymphocyte infiltration of the liver during the development of GVHD, differences in $T_{\text {eff }}$ cell transcriptional profiles might be associated with strain specific factors that determine susceptibility to liver inflammation. ${ }^{(292)}$

Analysis of the differentially expressed genes involved in the SLO-GVHD target organ dichotomy revealed fundamental differences in cellular programs, suggesting a greater level of effector differentiation in the GVHD target tissues (Figure 26 and Table 12). This unexpected level of heterogeneity in $T_{\text {eff }}$ cell differentiation according to location was also evident from the pattern of expression of effector genes (Figure 28), which suggested a preferential Tc1/Tc17 differentiation program in the small intestine and in the skin $\mathrm{T}_{\text {eff }}$ cells, contrasting to Tc2 differentiation in the liver.

Lastly, the blockage of lymphocyte egress from the SLO and the comparison of the transcription profiles of GVHD target tissues with the corresponding draining lymph nodes and blood subset populations refuted the hypothesis that the tissue-associated variability in gene expression could be explained by early trafficking of effector cells or by differential homing imprinting of donor T cells.

Altogether, the data suggest that the diversity in transcriptional profiles of donor $\mathrm{CD}^{+} \mathrm{T}_{\text {eff }}$ cells observed after an allogeneic BMT, at the peak of the alloreactive immune reaction, does not reflect differences in antigen expression 
between tissues nor an imbalanced distribution of particular clonal populations. The divide between the SLO and the GVHD target organs seems to be indicative of meaningful disparities in biological processes occurring at each site, and denotes the existence of a bias in the functional status of donor CD8 ${ }^{+}$ $\mathrm{T}_{\text {eff }}$ cells which is dependent on the tissues they infiltrate.

Dissection of the data set and identification of biologically meaningful tissue-specific transcriptional signatures that may be applicable to other models of GVHD will require the application of unbiased methods of systems biology, which will be addressed in Section 2 of the results chapter. 


\section{2. $\mathrm{T}_{\text {eff }}$ cell transcription profiles show compartmental specificity in acute GVHD}

To obtain a more global view of the transcriptional changes in the donor $\mathrm{CD}^{+} \mathrm{T}$ cell response in the context of acute GVHD development, weighted gene correlation network analysis (WGCNA) was used to identify differences in the gene expression patterns at each site.

WGCNA has been described as "a step-wise data reduction technique, which (a) starts from the level of thousands of variables, (b) identifies biologically interesting modules based on a node significance measure, (c) represents the modules by their centroids (e.g., eigenvectors or intramodular hubs), (d) uses intramodular connectivity as quantitative measures of module membership, and (e) combines node significance and module membership measures for identifying significant hub nodes".(293)

The discovery of highly correlated clusters of genes in an unsupervised manner is considered to be extremely useful computational approach in identifying modules of both co-expressed and co-regulated genes, ${ }^{(294-296)}$ which frequently represent conserved molecular "circuits" responsible for specific cellular functions. ${ }^{(297)}$ 


\subsection{Identification of gene clusters based on correlation and co-expression across tissues using WGCNA}

In order to avoid any potential bias related to potential heterogeneity of the $\mathrm{CD}^{+} \mathrm{T}$ cell populations isolated from the different sites, the WGCNA algorithm was applied on the data set derived from the MataHari T cell model after exclusion of outlier samples and of tissue specific genes, as defined in the PaGenBase database. ${ }^{(247)}$

The gene correlation network was constructed based on the pairwise Pearson correlations between all genes, by raising the co-expression similarity to a power $\beta$ chosen using the scale-free topology criterion, while retaining the information on the correlation signs between genes. The soft threshold 8 was selected as being the smallest value of $\beta$ with which approximate scale free topology is reached (Figure 34).

The correlation expression values were further used to construct a topological overlap matrix (TOM), which measured the proximity between neighbouring genes. Hierarchical clustering of the genes was performed using the dissimilarity values "1-TOM" as the distance measure. Modules were defined using a dynamic tree-cutting algorithm, merging those with a correlation above 0.75 (Figure 35). All uncorrelated genes were assigned to a grey module. 
A total of 31 highly correlated modules of genes were identified (Table 13). To study the relationships among the found modules, the first principal component of each module (eigengenes) was determined and used as its representative profile. The modules similarity was quantified by eigengene correlation and displayed as a dendrogram and a heat map (Figure 36).

Three major groups of correlated eigengenes, meta-modules, can be recognised: $(\mathrm{A})$ comprising modules 1 - 9; (B) comprising modules 10 -19; and $(C)$ comprising modules $20-31$.

To understand the biological meaning of these findings, the correlation between the modules and the sample traits (experimental groups: donor, synBMT, alloBMT; alloBMT tissue groups: SLO, GVHD target organs; individual tissues: lymph nodes, spleen, bone, blood, liver, gut - IEL, gut - LP, skin - dermis, skin - epidermis) was performed (Figure 37).

Indeed, a positive correlation between the meta-modules and the experimental groups was observed: meta-module B related to donor the synBMT samples, while modules $A$ and $C$ related to alloBMT samples (SLO and GVHD target organs, respectively). Moreover, unique patterns of correlation were distinguishable at the tissue level; the modules with the highest significance ( $p$-value $\leq 0.05)$ being:

- lymph nodes - modules 5, 8 and 17;

- $\quad$ spleen - modules 2, 3, 4 and 6;

- blood - modules 1 and 20; 
- $\quad$ bone marrow - modules 1, 3 and 4;

- $\quad$ liver - module 4;

- gut (LP) - modules 9, 13 and 27;

- gut (IEL) - modules 6, 29, 30 and 31;

- $\quad$ skin (dermis) - modules 22, 23, 25, 26 and 29;

- $\quad$ skin (epidermis) - modules 26, 28 and 29.

To better visualise the relationships between the modules, a eigengene network was generated using the modules as nodes and the inter-module adjacency measure as edges (Figure 38). This representation illustrates the diversity of the transcriptional signatures, highlighting the similarity between donor and the synBMT transcriptional profiles (green and blue) and the dichotomy between the alloBMT SLO (yellow) and GVHD target organs (orange) gene expression. 
A

Scale independence

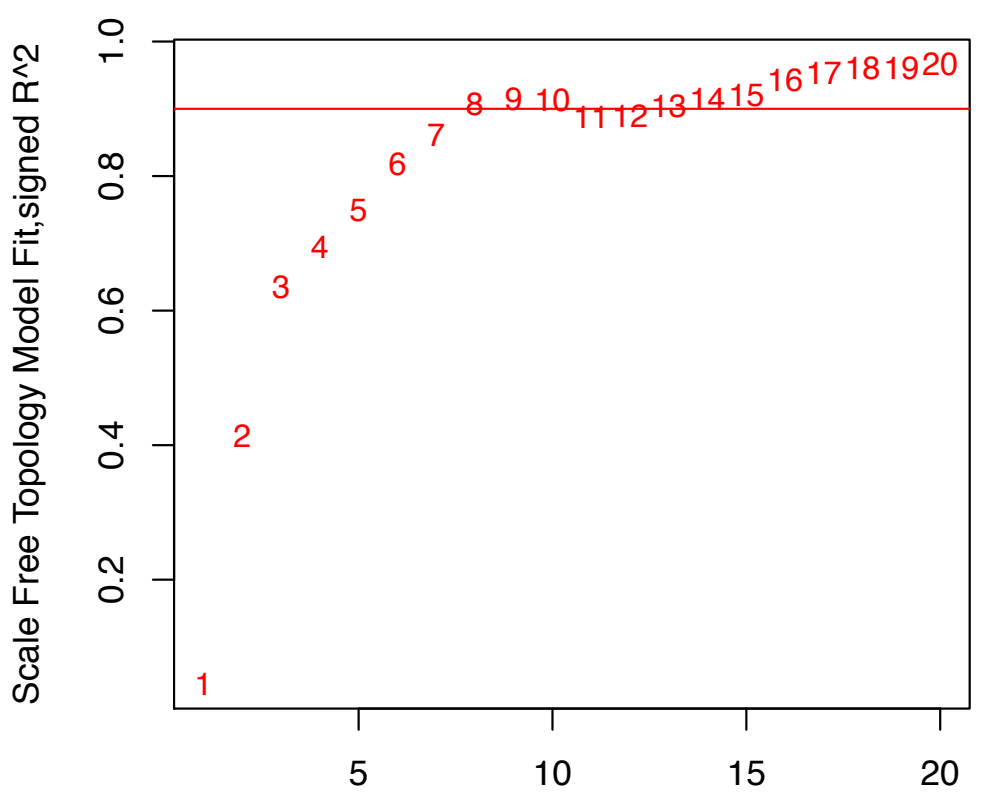

Soft Threshold (power)

B

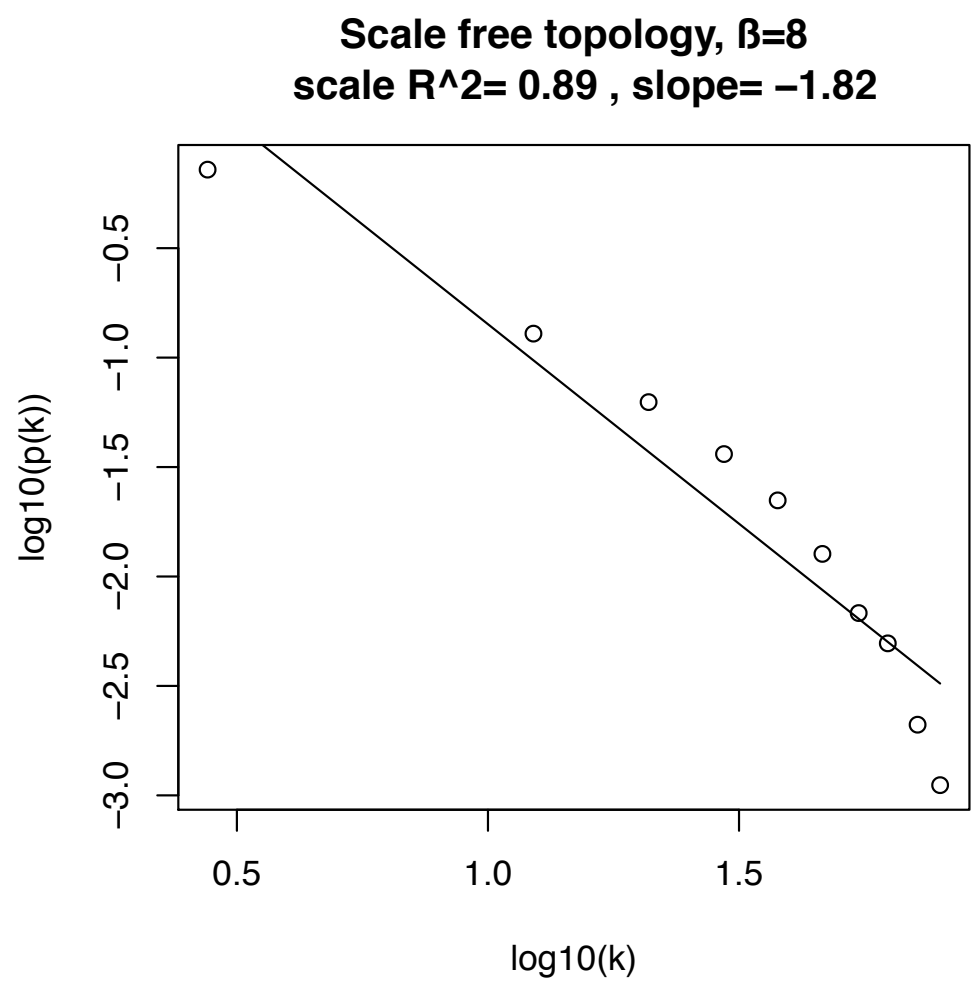

Figure 34. Selection of the power $\beta$ for gene correlation network generation. (A) Plot showing the scale-free fit index as a function of the soft-thresholding power. Soft threshold 8 is the lowest power to achieve a scale-free fit index above 0.90 (red line). (B) Log-log plot of the network connectivity using soft threshold power 8 . The approximate straight line relationship (high $\mathrm{R}^{2}$ value) shows approximate scale free topology. 


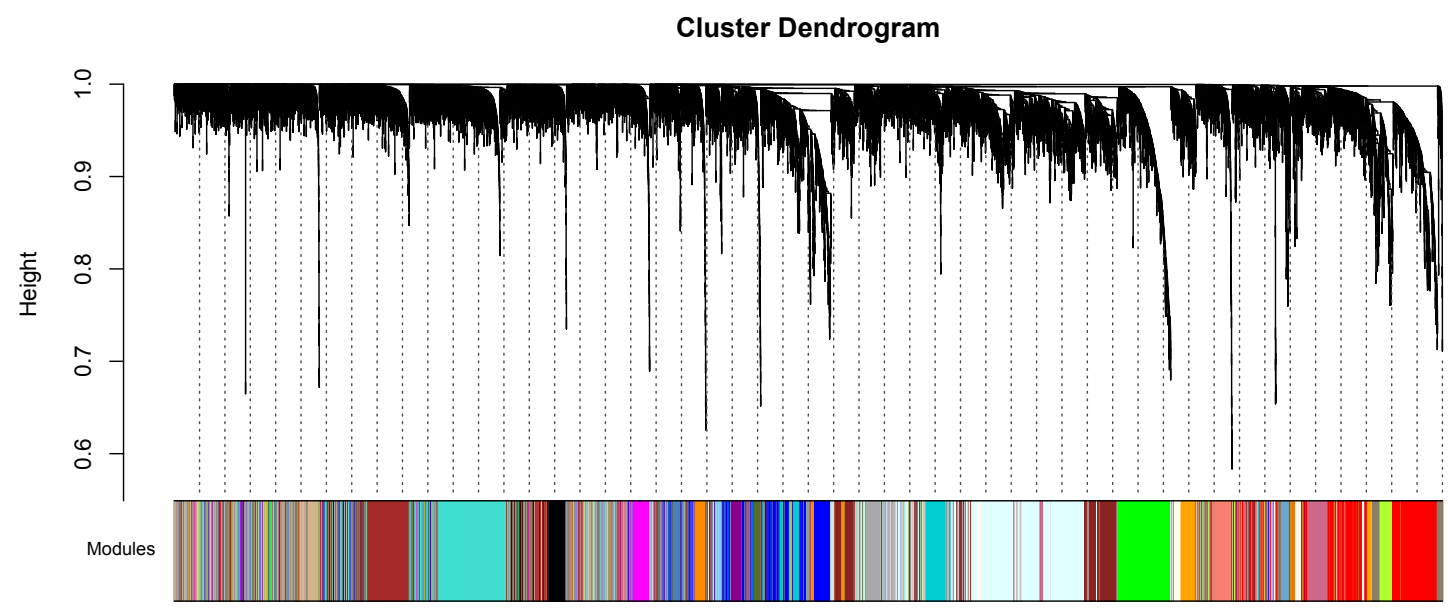

Figure 35. Dendrogram obtained by hierarchical clustering of genes based on topological overlap, showing the assigned modules colour-coded.

Table 13. WGCNA modules.

\begin{tabular}{cllcclcc}
\hline Mod. & Colour code & No. of genes & & Mod. & Colour code & No. of genes \\
\hline \hline 1 & green & 816 & & 17 & darkturquoise & 574 \\
2 & brown4 & 1113 & & 18 & skyblue & 179 \\
3 & lightcyan & 615 & & 19 & steelblue & 158 \\
4 & lightcyan1 & 1569 & & 20 & magenta & 381 \\
5 & skyblue3 & 117 & & 21 & brown & 913 \\
6 & floralwhite & 151 & & 22 & tan & 345 \\
7 & orange & 298 & & 23 & black & 559 \\
8 & ivory & 90 & & 24 & turquoise & 1384 \\
9 & palevioletred3 & 327 & & 25 & salmon & 336 \\
10 & darkorange & 481 & & 26 & white & 186 \\
11 & darkolivegreen & 134 & & 27 & red & 1520 \\
12 & grey60 & 283 & & 28 & bisque4 & 443 \\
13 & lightsteelblue1 & 103 & & 29 & greenyellow & 363 \\
14 & blue & 1100 & & 30 & darkgrey & 206 \\
15 & yellowgreen & 124 & 31 & darkorange2 & 86 \\
16 & darkmagenta & 131 & & & \\
\hline
\end{tabular}




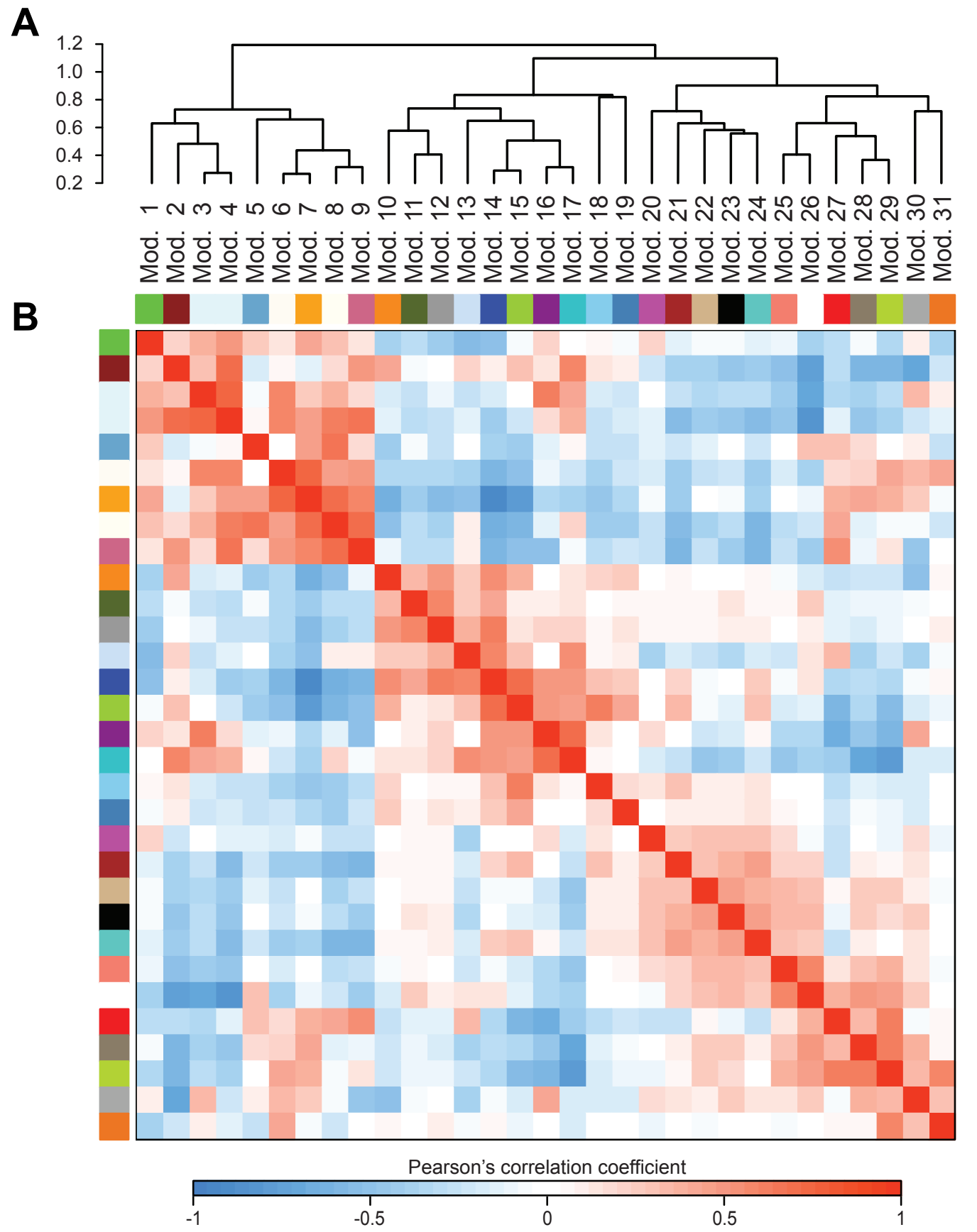

Figure 36. Visualization of the eigengene network representing the relationships among the modules. (A) Hierarchical clustering dendrogram of the eigengenes based on dissimilarity measure. (B) Heat map showing the correlation between eigengenes. 


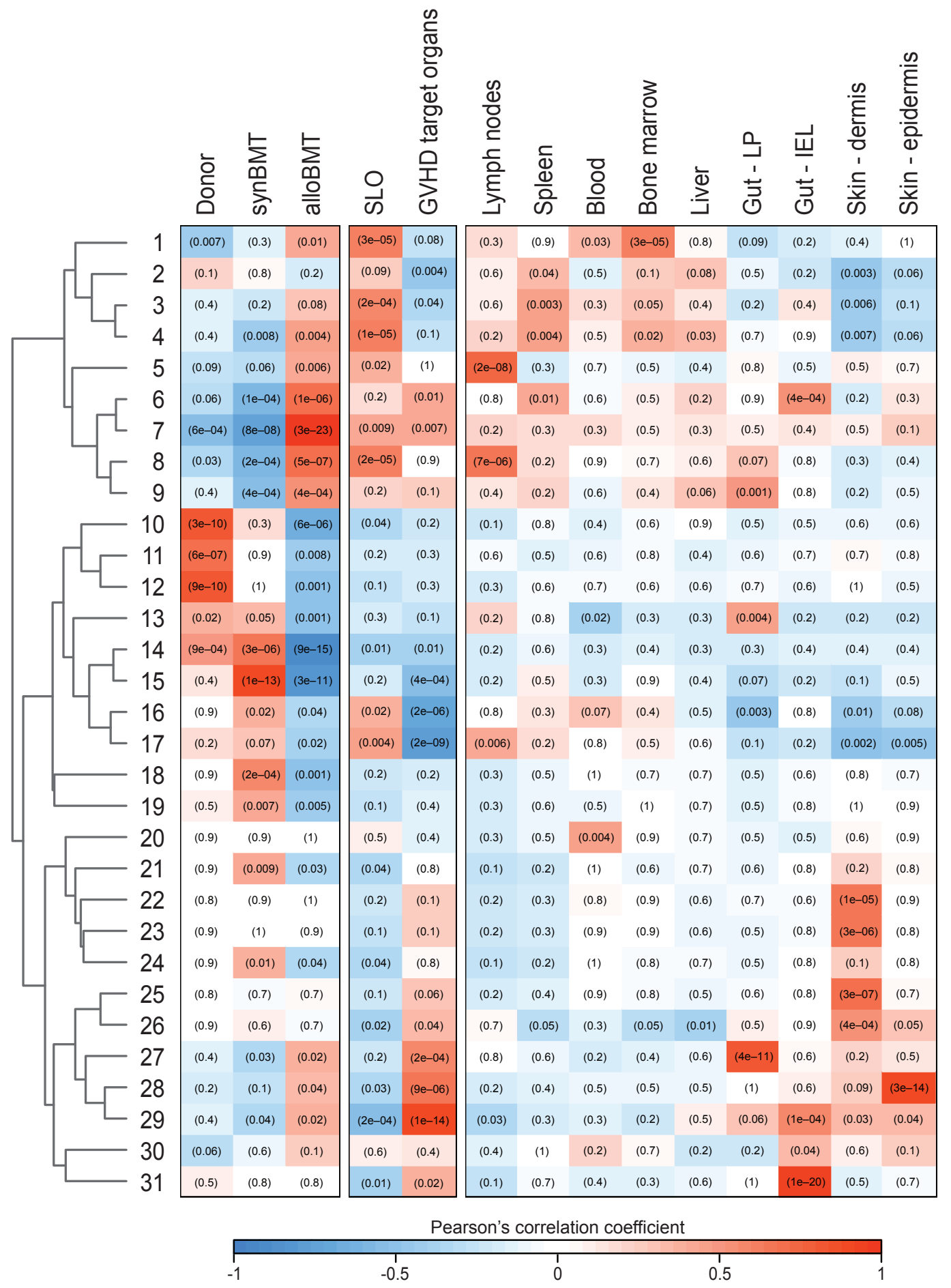

Figure 37. Heat map representation of the module-trait relationships. The table is colour-coded by correlation according to the colour label and each cell contains the corresponding $p$-value. 


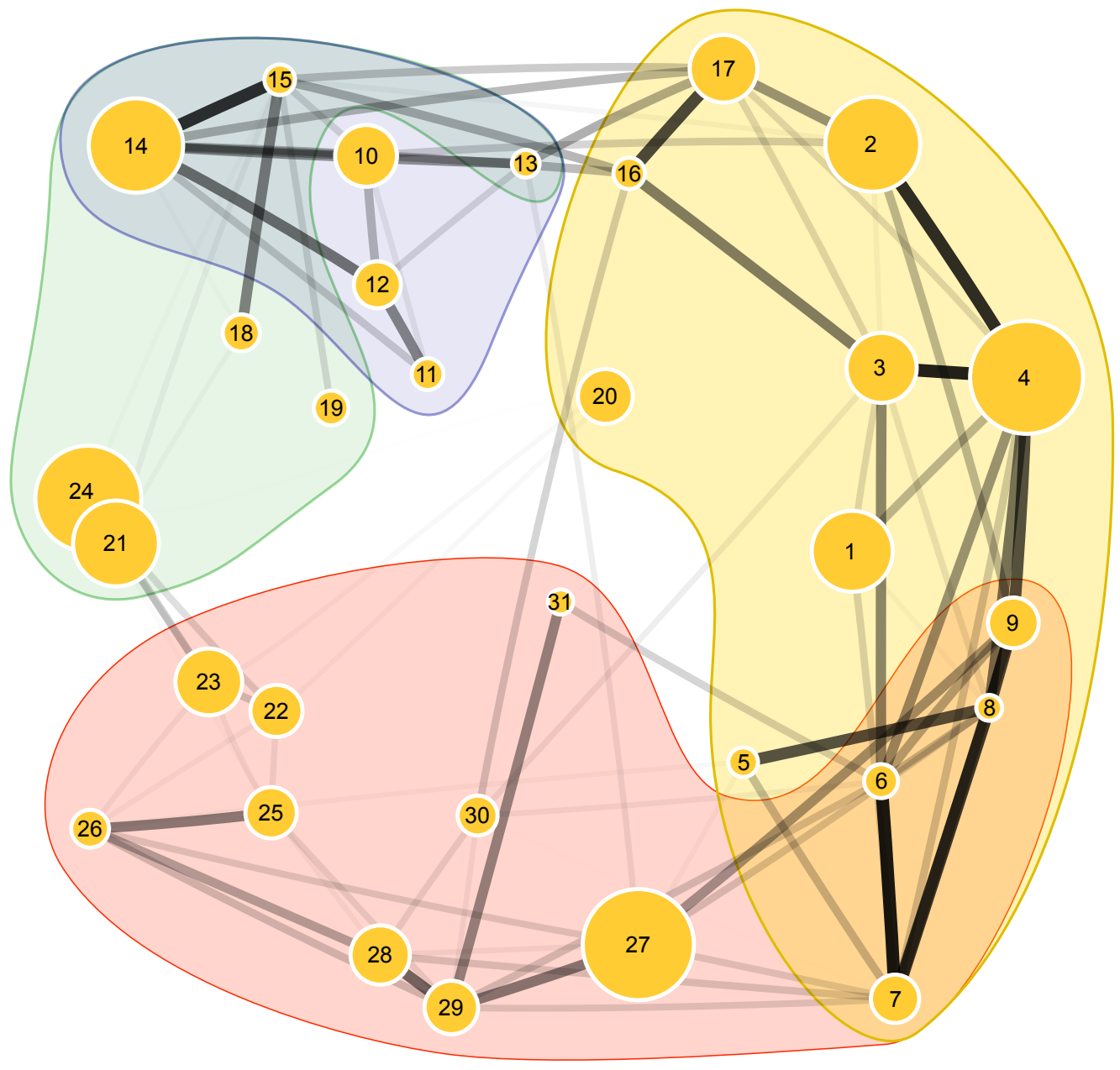

Figure 38. Eigengene network generated using the edge-weighted spring embedded layout algorithm (weight based on the inter-module adjacency measure, displayed as the relative distance between nodes; node area proportional to the size of the module; edge thickness proportional to the inter-module correlation coefficient; edge darkness proportional to the statistical significance of the correlation; edges with $p$-value $>0.05$ not displayed). 


\subsection{WGCNA defined modules are preserved in an independent murine model of GVHD}

Before advancing to the in-depth characterisation of the individual modules, the reproducibility of this modular organisation of the transcriptional profiles was tested by evaluating module preservation in an independent test data set $(\mathrm{B} 6 \rightarrow 129$ model). For this purpose, the "modulePreservation" function from the WGCNA R package ${ }^{(261)}$ was used, which calculates a composite $Z_{\text {summary }}$ statistic, taking into consideration both the network density and connectivity to determine whether the nodes remain highly connected in the test network, and if the connectivity pattern in the test network is similar to the reference network. Figure 39 summarises the results from this module preservation study.

According to the threshold guidelines recommended by Langfelder ${ }^{(261)}$ $\left(Z_{\text {summary }} \geq 10\right.$ : "strongly preserved" modules; $2 \leq Z_{\text {summary }}<10$ : "weak to moderately preserved" modules; $Z_{\text {summary }}<2$ : "not preserved" modules), 19 out of the 31 modules were strongly preserved (modules $1,2,3,4,6,7,9,13,14$, $15,17,23,24,26,27,28,29,30,31)$ and only 1 module was not preserved (module 5). 


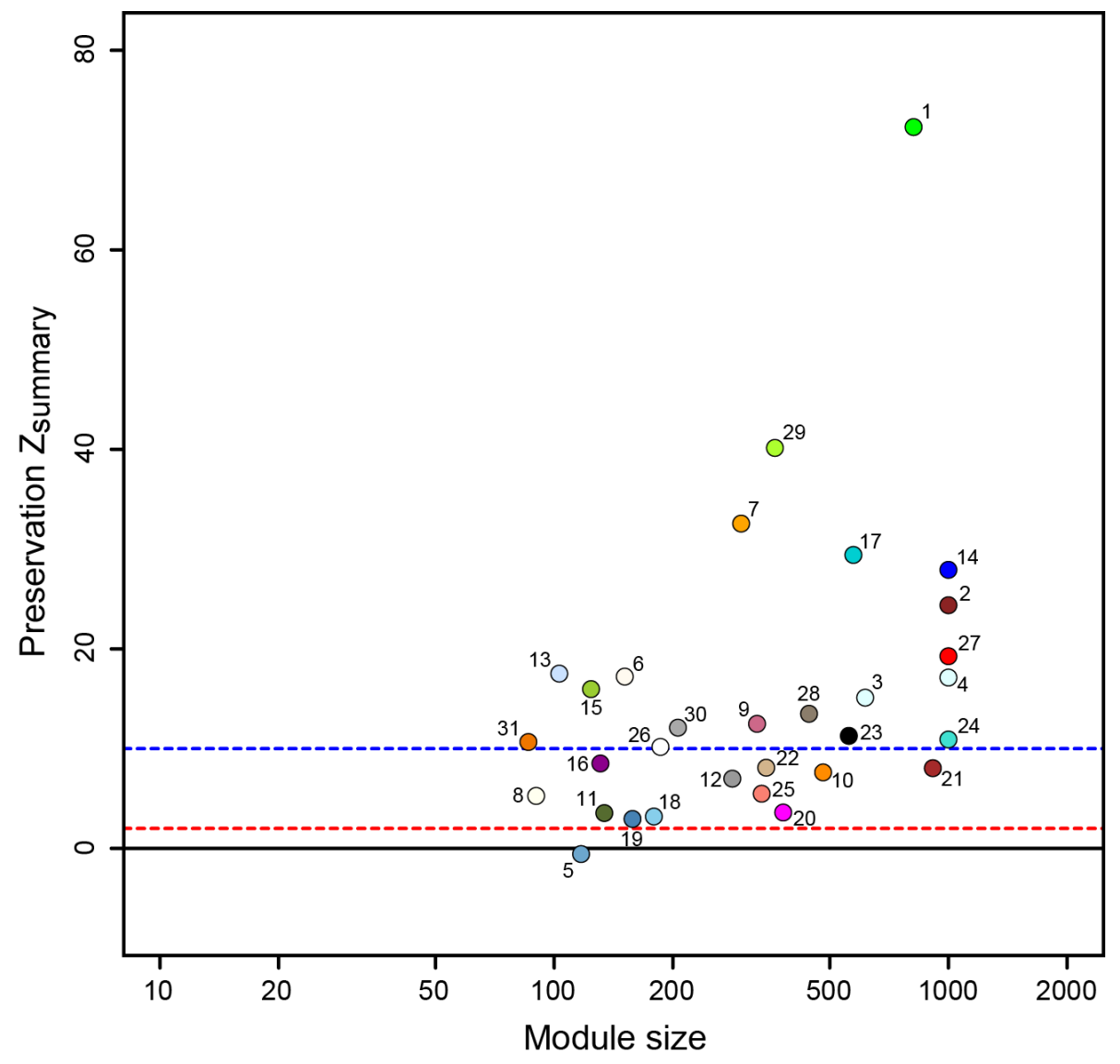

Figure 39. Module preservation study. The plot shows the summary statistics $Z_{\text {summary }}$ as a function of the module size (blue line: $Z_{\text {summary }}=10$; red line: $Z_{\text {summary }}=2$ ). 


\subsection{Organ specific modules are conserved across species}

To validate the modules further and assess their potential translational relevance, GSEA was run on two published and publically available data sets of gene expression profiling from peripheral blood $\mathrm{CD}^{+} \mathrm{T}$ cells of rhesus macaques (GSE73723) and humans (GSE73809) developing GVHD following allogeneic BMT, ${ }^{(298)}$ using the strongly preserved modules as gene sets.

In rhesus macaques, module 1 was found to be strongly associated with the GVHD samples from untreated alloBMT recipients (Figure 40-A), while module 13 was associated with the synBMT control samples (Figure 40-B). Interestingly, the enrichment for module 1 was lost in the samples from alloBMT recipients treated with sirolimus (Figure $\mathbf{4 0 - C}$ ) or with tacrolimus-methotrexate (Figure 40-D), reinforcing the relevance of this module in GVHD biology.

In the human data set, where samples from patients with and without GVHD were compared, there was a clear segregation of the SLO-associated modules (modules 1, 2, 3, 4, 6 and 17) which were enriched in the GVHD group (Figure 41), while the only gene set enriched in the non-GVHD group was module 24, a synBMT-associated module (Figure 42).

Since these two data sets were constructed using only blood samples, the preservation of the GVHD target organ-associated modules was not possible to determine in this analysis. The lack of published data examining the gene expression of donor T cells in the peripheral tissues both in animal models 
and in humans highlighted an important gap in the current knowledge of GVHD.

To explore this research niche, a collaboration with Dr Laura Jardine and Dr Mathew Collin from the Newcastle University Institute of Cellular Medicine was initiated with the objective of studying the transcriptional profile of $\mathrm{CD}^{+} \mathrm{T}$ cells isolated from the blood and skin of transplanted patients with GVHD. Being an ongoing project, the results included in this thesis are based only the analysis of the first 4 patients.

Due to the low number of cells isolated from the tissue samples, gene profiling was performed using the deep-sequencing technology RNA-seq. Calculation of read counts, normalization and differential expression analysis was performed using the Basespace Illumina tool Cufflinks Assembly \& DE (https://basespace.illumina.com/apps/2582581/Cufflinks-Assembly-DE).

Given the small number of samples and the heterogeneity of the patients' background (different acute GVHD severities, variable lengths of time from first GVHD signs and symptoms until sample collection, concurrent comorbidities and treatments) the strategy employed for the analysis of the data involved the computation of the single-sample GSEA (ssGSEA) for the 31 modules identified in the murine data set. This methodology is an extension of GSEA allowing the definition of an enrichment score that represents the degree of absolute enrichment of a gene set in each sample within a given data set. ${ }^{(265)}$ To establish the relative enrichment of the modules in each compartment, the NES were compared across the paired blood, dermis and epidermis samples for the individual patients. 
Figure 43 is a heat map representation of this comparison. From its analysis it becomes evident the existence of a pattern in module enrichment that, in 3 of the 4 patients, successfully distinguished between the samples derived from the epidermis or from the blood. Although in patient $A$ the pattern is not present, it is noteworthy that the epidermis-specific module 28 and the pan-GVHD target organ-associated module 29 are invariably overrepresented in the samples from the epidermis, in all patients.

Interestingly, the pattern of module enrichment in the dermis was considerably similar to that of the blood. Although this could suggest that, in humans, the dermis compartment is functionally very different to that of mice, it is also possible that this result could just have identified a pitfall in the processing methodology. In contrast to the epidermis, the dermis is a highly vascularised tissue; the impracticability of flushing the vasculature of the biopsy specimens may cause substantial contamination of the dermis samples with circulating lymphocytes, which could bias the analysis towards a more blood-like signature.

Altogether, these results corroborate that the modules found in the MataHari T cell model are preserved and conserved across species, which suggests they correspond to biologically meaningful sets of genes. 
A

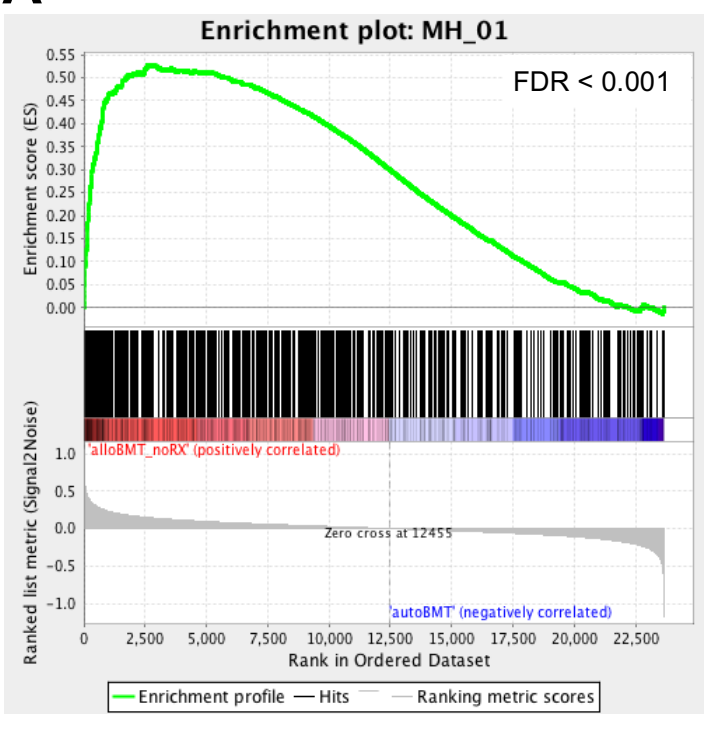

C

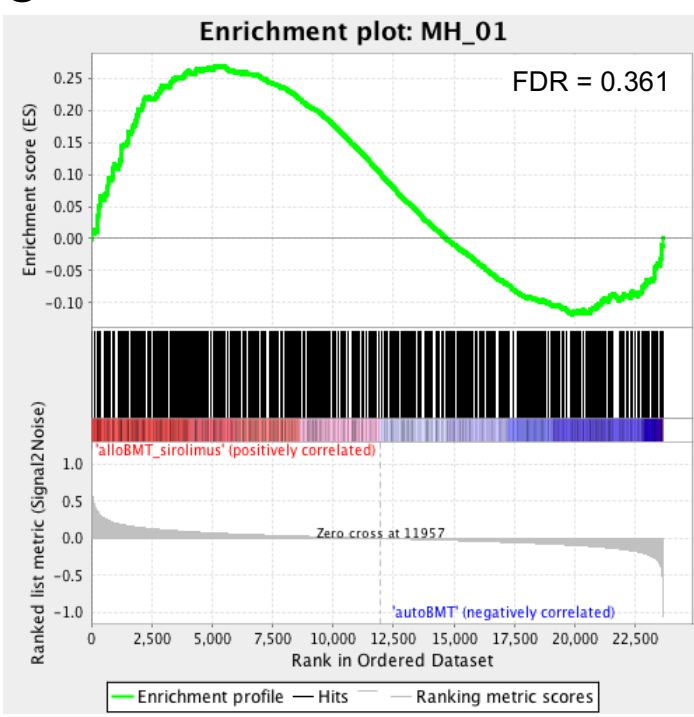

B

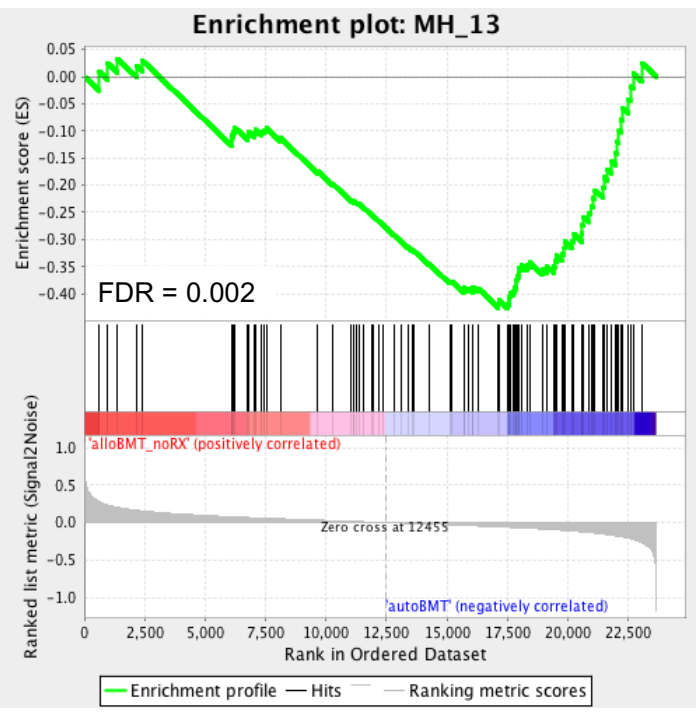

D

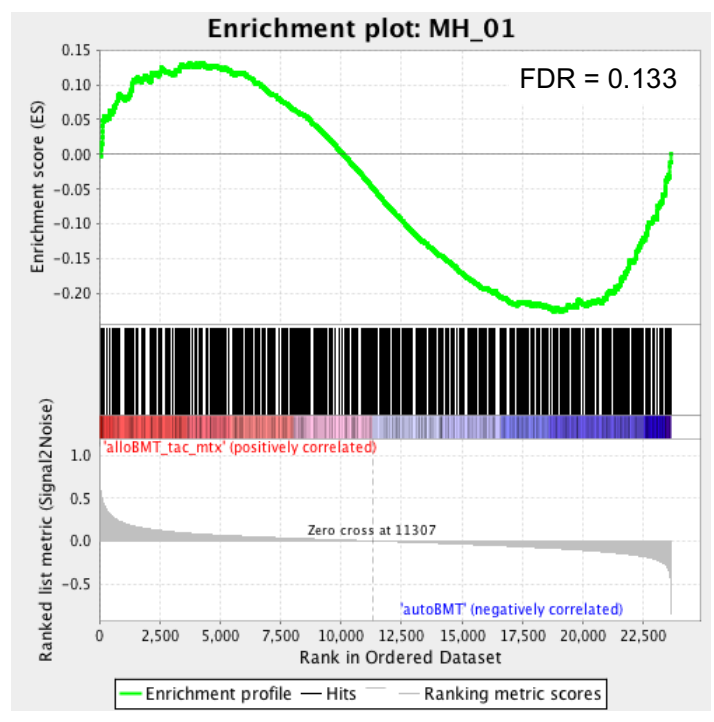

Figure 40. Validation of the murine modules on a data set from rhesus macaque. GSEA was performed to compare profiles of untreated alloBMT versus synBMT samples (A, B), sirolimus treated alloBMT versus synBMT samples (C), and tacrolimus-methotrexate treated alloBMT versus synBMT (D). Note the statistically significant enrichment for module 1 is observed in the untreated alloBMT samples (A), which is lost upon immunosupressive treatment (C, D). 
A

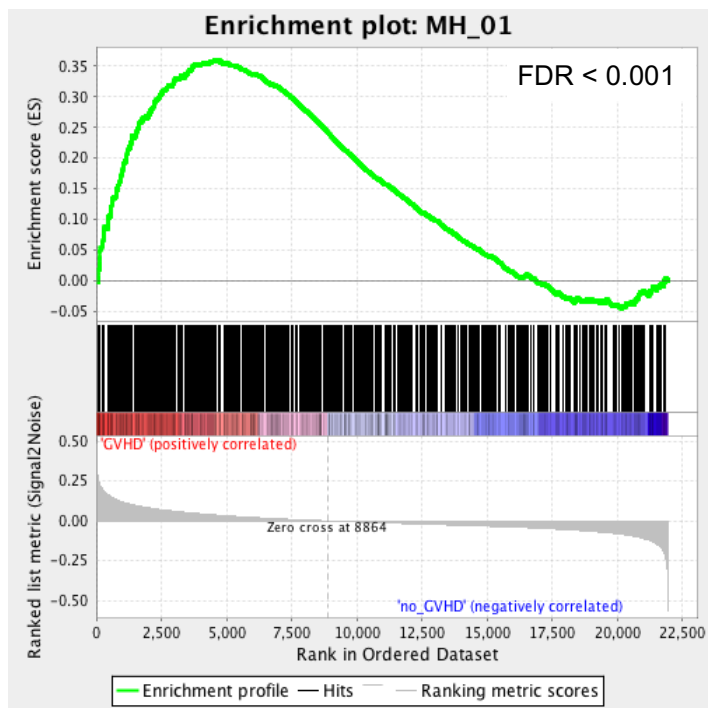

C

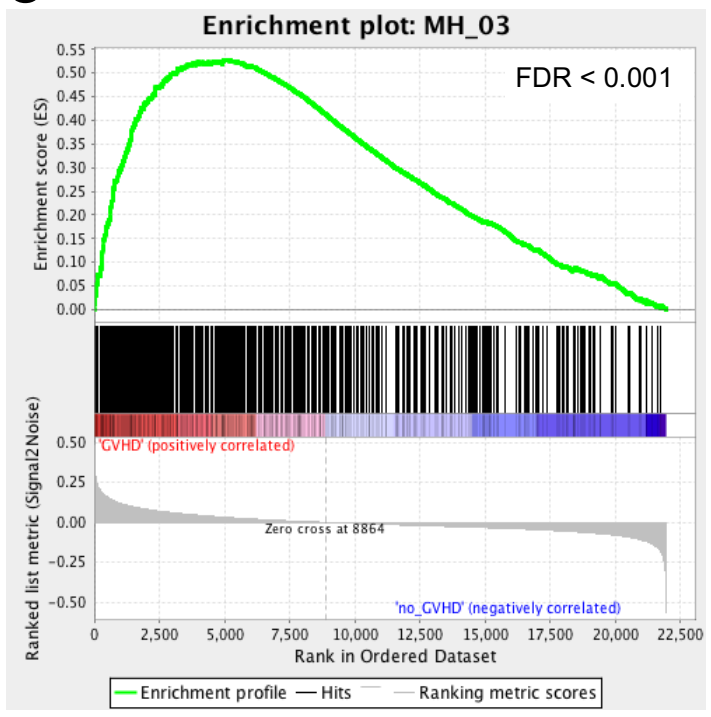

$\mathbf{E}$

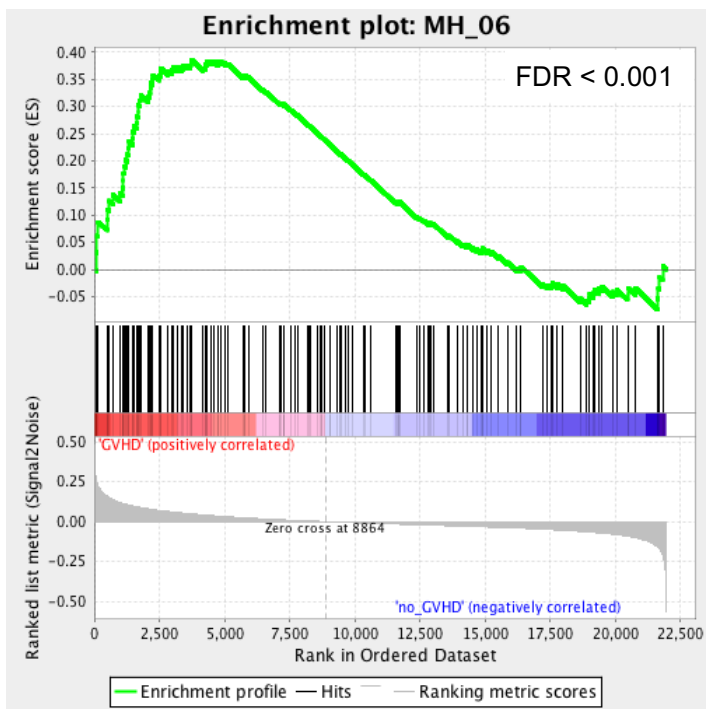

B

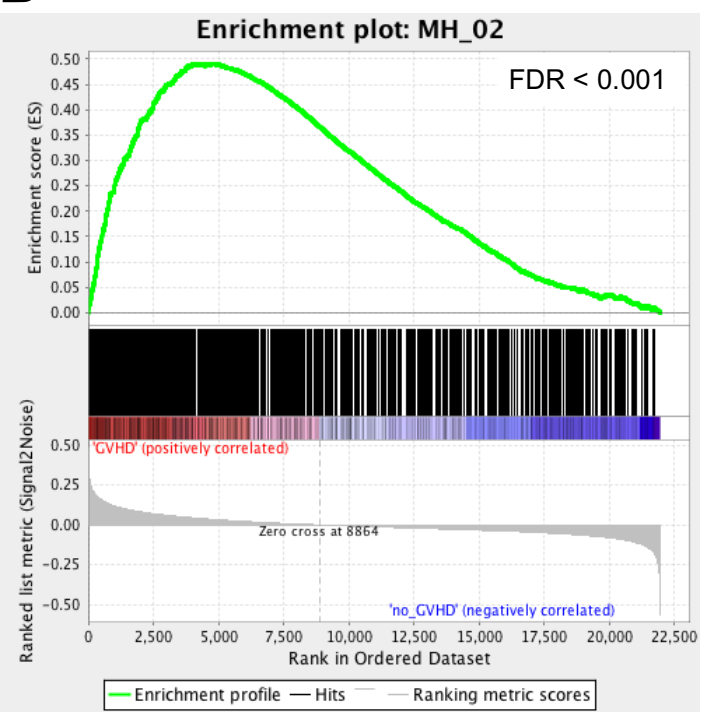

D

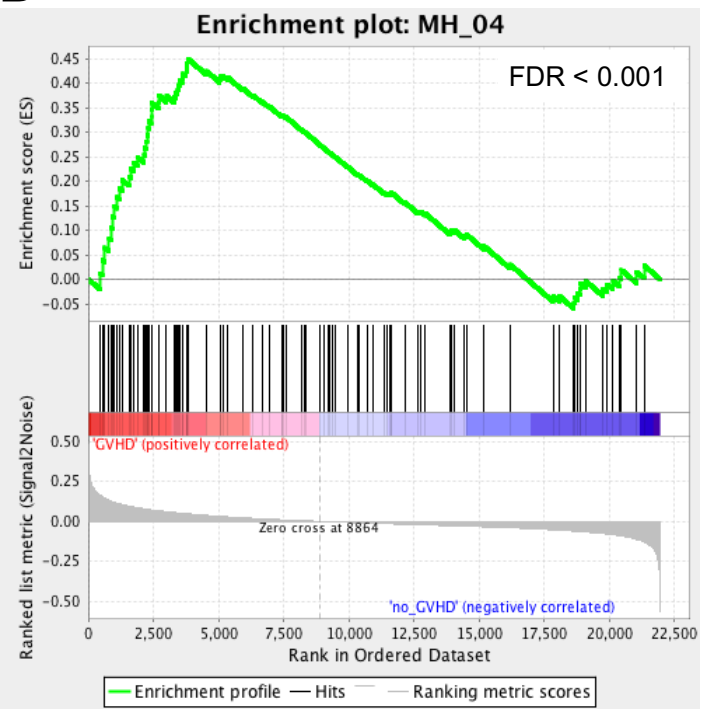

$\mathbf{F}$

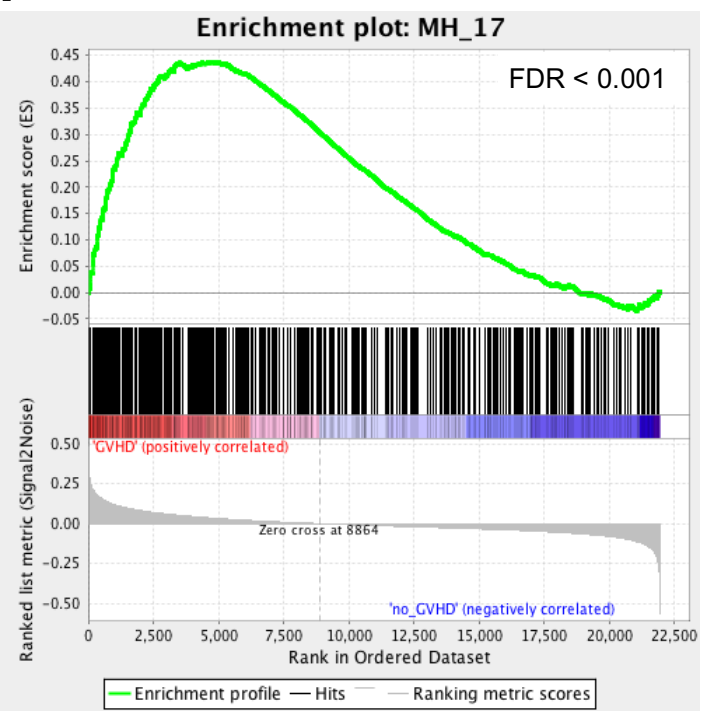

Figure 41. Validation of the murine modules on a data set from human samples. 
Figure 41 (previous page). Validation of the murine modules on a data set from human samples. GSEA was performed to compare profiles of samples from transplanted patients with and without GVHD. (A - F) Statistically significant enriched modules in the GVHD samples. Note that all the enriched gene sets $(1,2,3,4,6$ and 17) are SLO-associated modules.

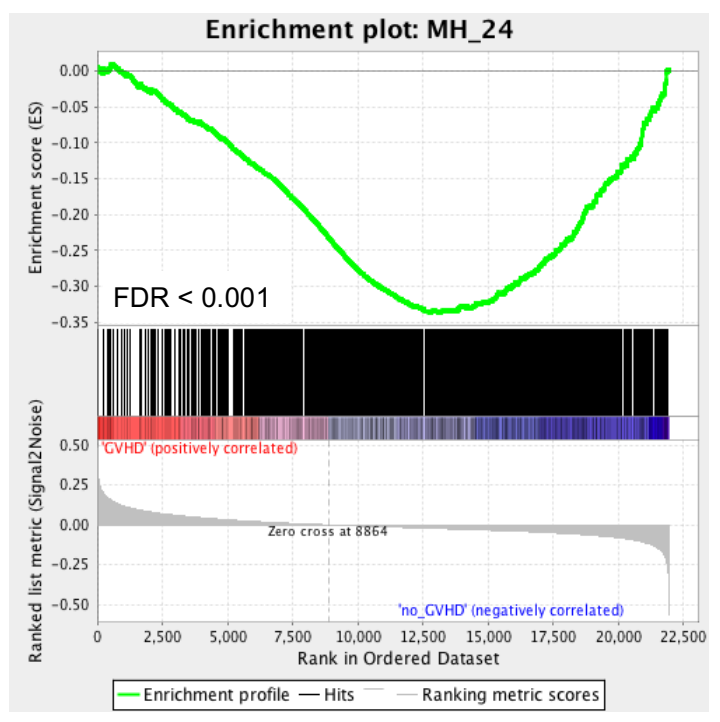

Figure 42. GSEA revealed statistically significant enrichment for module 24 in the samples from patients without GVHD. 


\section{Patient A Patient B Patient C Patient D}

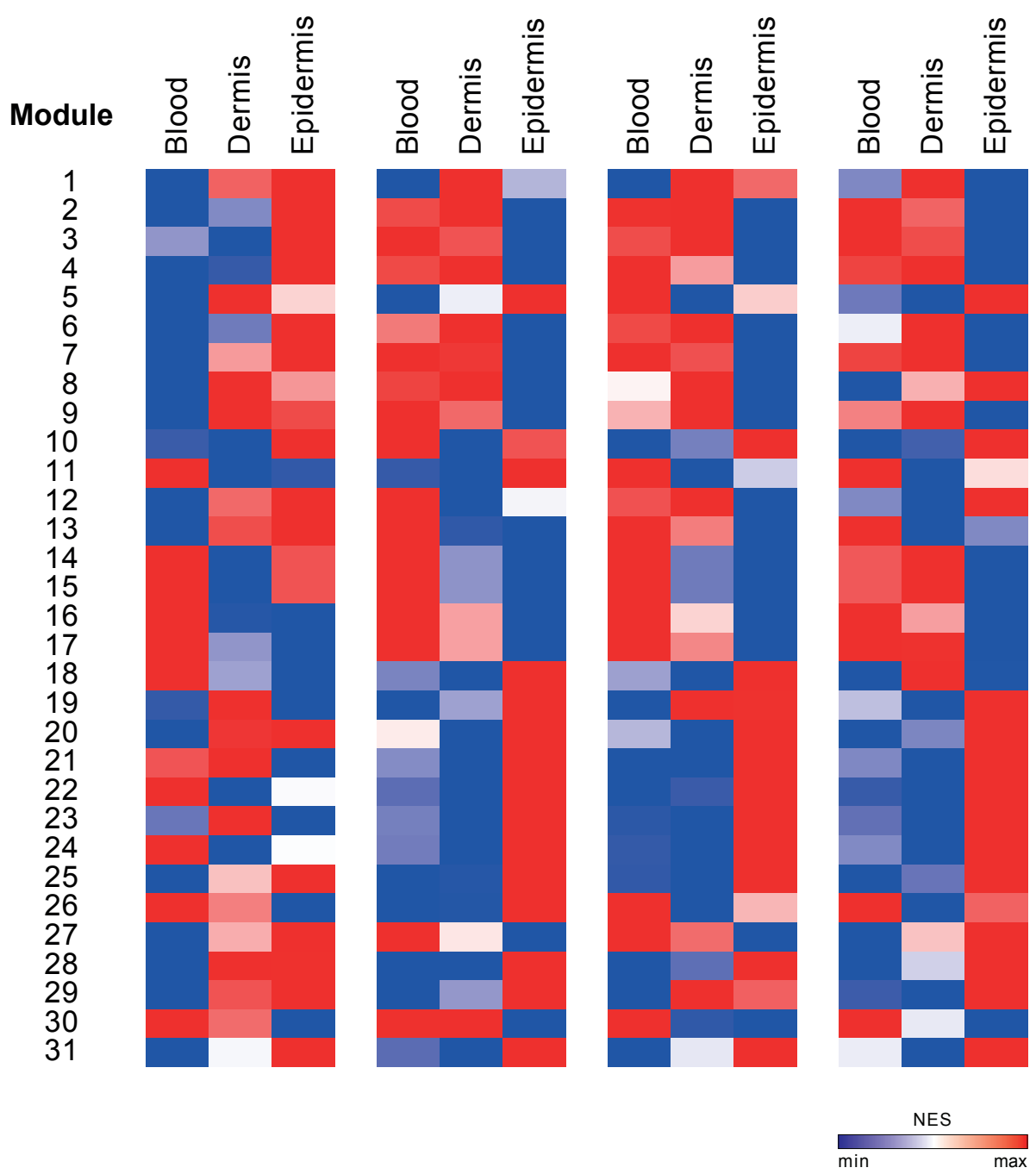

Figure 43. GVHD target organ-associated modules accurately distinguish between samples isolated from the epidermis and samples isolated from the blood of transplanted patients with cutaneous acute GVHD. The heat maps show normalised enrichment score (NES) for each gene set determined by ssGSEA of blood, dermis and epidermis paired samples from 4 different patients. 


\subsection{Module annotation reveals biologically meaningful clusters of genes}

To evaluate the functional relevance of coexpressed genes within the 19 strongly preserved modules, WebGestalt (WEB-based GEne SeT AnaLysis Toolkit) ${ }^{(263,264)}$ was used to examine the overrepresentation of the Gene Ontology (GO) categories (biological processes, molecular function, and cellular component) (Appendix - Supplementary Figures 2 - 20) and KEGG pathways (Appendix - Supplementary Table 2) within each module. The analysis of these results indicated that:

- modules 13, 14 and 15, associated both with donor and synBMT samples, were related with epigenetic regulation of gene expression (by chromatin organisation and histone modification), nucleic acid biosynthesis, and organisation and biogenesis of cellular components;

- module 7, associated with all alloBMT samples, was related with T cell activation and migration;

- module 17, associated with alloBMT LN samples, was mostly related with immune response modulation by Toll-like receptor and protein metabolism regulation by the mTOR pathway;

- modules 2, 3 and 4, associated with alloBMT spleen samples, were related with cellular component biogenesis and organisation, protein biosynthesis, and energy production through oxidative phosphorylation; 
- module 1, associated with alloBMT bone marrow and blood samples, was related mainly with regulation of the cell cycle, nucleotide metabolism, and organelle organisation;

- module 29, associated with all GVHD target organs, was related primarily with cell differentiation and regulation of apoptosis, in response to TCR and MAPK signalling;

- modules 9, 13 and 27, associated with alloBMT gut (LP) samples, were related with epigenetic regulation of transcription (through chromatin organisation and histone modification) and regulation of protein metabolism by the ubiquitin-proteasome system;

- modules 6, 30 and 31, associated with alloBMT gut (IEL) samples, were related with $\mathrm{T}$ cell migration and activation;

- modules 23 and 26, associated with alloBMT skin (dermis) samples, were related with cell migration and differentiation, and oxidative phosphorylation;

- module 28, associated with alloBMT skin (epidermis) samples, were mainly related with regulation of the inflammatory response.

This modular representation of donor $C D 8^{+} \mathrm{T}_{\text {eff }}$ cell gene expression further highlights the similarities between donor and synBMT samples, and emphasises the SLO-GVHD target organ transcriptome dichotomy revealed in the exploratory analysis of the data. In the steady state and after a synBMT, the donor T cells appear to be in quiescent state dominated by cell cycle regulation. However, in the context of an alloBMT a number of different cellular programs are activated, some of which are broadly expressed across all organs, while others are discretely associated with specific tissue compartments. 


\subsection{Identification of highly connected hub genes}

According to the theory of module networks, the genes with the highest degree of connectivity within a module (known as hub genes) are interpreted as being representative of that module and are expected to be drivers required for core cellular pathways.

Module specific driver genes were identified using the eigengene-based connectivity, as a module membership measure, and their correlation with the sample traits, as a measure of gene significance (Apendix - Supplementary Table 3), and calculation of the over-enriched GO terms was performed using WebGestalt $^{(264)}$ (Apendix - Supplementary Figures 21 -39). Table 14 summarises these results, showing the top 10 driver genes in each module and the top 3 over-represented GO terms.

Integration of the information acquired from the module-trait relationship analysis, GO and KEGG pathway enrichment study and driver genes identification revealed biologically relevant tissue-specific features that reflect distinct stages of differentiation and activation, metabolic status, and cell fates of donor $\mathrm{CD} 8^{+} \mathrm{T}_{\text {eff }}$ cells.

Consistent with the current model of GVHD immunopathology, ${ }^{(165)}$ the pan-alloBMT associated gene set, module 7, includes key genes essential for T cell activation, expansion and migration, such as CD28 and CD4OL, Tbx21 and Itga4. Moreover, the modules associated with SLO appear to mainly be 
related to priming and proliferation cellular programs. Modules 1,3 and 4 , a common SLO transcriptional signature, are linked to cell cycle, DNA replication and oxidative metabolism, including genes like Cdc26, Cdk7, Ccnh, Atr, Hadh, Cs, Mdh1, Mdh2, and numerous others encoding proteins of the electron transport chain. Of relevance, module 17 appears to mark the contrast between SLO and GVHD target organs, being highly positively correlated with the LN but negatively correlated with both the gut and the skin. Interestingly, module 17 is mostly related to Toll-like receptor signalling (e.g. TIr1, TIr3, TIr7, Irf7, Ifnar2, Irak4), stressing the importance of co-stimulation complementary to TCR-induced signals to enhance $\mathrm{T}_{\text {eff }}$ cell proliferation, survival and cytokine production in the lymphoid organs.

The GVHD target organ-associated modules, on the other hand, have an overwhelming number of genes linked to pathogenicity both through direct cytotoxicity and pro-inflammatory cytokine generation. In this context, module 29 could be viewed as a common transcriptional signature associated with all GVHD target organs, which is related to regulation of cell fate decisions through mitogen-activated protein kinases (MAPK) and TNF-alpha NF-kB signalling, and include several genes that determine Tc1 and Tc17 differentiation (e.g. Tnfr2, Traf1, Traf4, Gadd45b, Nr4a2, Tnfaip3, Rel, Fos/2, Kdm6b, Skil, Chd7). Taking into consideration the gut-associated module 31, further Tc17 polarisation is expected to occur in gastrointestinal tract as indicated by expression of additional Th17/Tc17 differentiation-related transcription factors, such as Ikzf3. In contrast, the epidermis-specific module 28 was predominantly linked to $\mathrm{T}$ cell activation (II2ra, Tnfs9, Cd44), Th1-type pro-inflammatory 
cytokine generation (Ifng, Csf1 and Csf2), glycolysis (Pkm2), Notch signalling (Rpbj, Furin), and survival (Bc/2/1). 
Table 14. Module specific driver genes summary.

Mod. Top 10 driver genes

1 Arhgap19, Hist2h2ac, Ube2c, Ccnb2, Kif18b, Ckap2, Cep55, Cdc20, Depdc1a, Prr11

2 Gtpbp8, Psmg1, Bphl, Tomm70a, Eif3m, Znhit6, Thumpd3, Trmt5, Tfb1m, 2210016F16Rik

3 Lyrm2, Cst7, Dtd1, Bloc1s1, Eif1b, Rpl37a, Pop5, Tor2a, Ostc, 2700060E02Rik

4 Asap1, Pglyrp1, Nup37, Lclat1, Dera, Slamf6, Klhdc2, Tjp2, Pts, Nqo2

6 Fdft1, Tacc2, Srgap3, Fh/2, Itih5, Ctsb, Angpt/2, Nmb, Xc/1, Bc/2a1a

7 Zdhhc2, Nek7, Ptpn11, Cox17, Plek, S100a11, Farp1, Anxa2, Ccdc50, Plscr1

$9 \quad$ Nab1, Adss, Zwint, Slu7, Ddx52, Ugp2, Cast, Tars, Gramd1c, Hat1

13 Zmym2, Kmt2a, Usp32, Herc1, Bach2, Brwd1, Nipbl, Vps13d, Dync1h1, Ly75

14 Stat1, Nuak2, Mgat4a, Arl4c, Atp1b1, Tbxa2r, Syde1, Pik3ip1, Rapgef6, Galnt10

15 Rras2, G0s2, Eomes, Slc14a1, Ppargc1b, Utp20, Prmt3, Sh3bp1, Kat2a, Dkc1 top 3 over-represented GO terms

GO:0007049 - cell cycle

GO:0051301 - cell division

GO:0048285 - organelle fission

GO:0006996 - organelle organisation

GO:0051301 - cell division

GO:0008152 - metabolic process

GO:0044237 - cellular metabolic process

GO:0009060 - aerobic respiration

GO:0007005 - mitochondrion organisation

GO:0055114 - oxidation-reduction process

GO:0006091 - generation of precursor metabolites and energy

GO:0022900 - electron transport chain

GO:0035746 - granzyme A production

GO:0071613 - granzyme B production

GO:0032623 - IL-2 production

GO:0080134 - regulation of cell activation

GO:0032101 - regulation of response to external stimulus

GO:0051174 - regulation of phosphorus metabolic process

GO:0016070 - RNA metabolic process

GO:0006397 - mRNA processing

GO:0006348 - chromatin silencing at telomere

GO:0016569 - covalent chromatin modification

GO:0016570 - histone modification

GO:0006355 - regulation of transcription, DNA-dependent

GO:0048583 - regulation of response to stimulus

GO:0023051 - regulation of signaling

GO:0010646 - regulation of cell communication

GO:0022613 - ribonucleoprotein complex biogenesis

GO:0002302 - CD8-positive, alpha-beta T cell differentiation involved in immune response

GO:0034641 - cellular nitrogen compound metabolic process 
Table 14 (continued)

Mod. Top 10 driver genes

17 Slfn5, Samhd1, Herc6, Prkdc, Smchd1, Klh/6, Fchsd2, Fam208a, Atf7ip, Parp9

23 Cytl1, Asphd2, Mia, Tslp, Slc51a, Snord88a

24 Tmem52, Krtap19-3, Ccdc85a, Amy1, Cntnap2, Pcdhb1, Nefl, Bpifa2, Rhou, D930020B18Rik

26 Clk1, Ddx5, Srrm2, Gabbr1, Malat1, Nxf1, Ptbp2, Leng8, Cdk16, 2810403A07Rik

27 Ramp3, Mpp7, Arhgap31, Socs5, Zbtb46, Mest, Tnfsf4, Rnf157, Ubtd2, $A b c b 1 b$

28 Ifitm5, Cpd, Csf2, Ifng, Nek6, Tnfsf9, Tesk1, Csf1, Ccl20, Cd44

29 Dusp5, Traf1, Tnfaip3, Tgif1, Coq10b, Rgs1, Nfkbiz, Dusp4, Smim3, Gpr132

30 Arf6, Sh3bgrl3, Bak1, Sec61a1, Mapk1ip1I

31 Slc16a10, Ikzf3, Asb2, Cd38, P2rx7, Chn2, Osgin1, 1810011H11Rik top 3 over-represented GO terms

GO:0050776 - regulation of immune response

GO:0006952 - defense response

GO:0032753 - positive regulation of IL-4 production

(*)

GO:0032536 - regulation of cell projection size

GO:0043484 - regulation of RNA splicing

GO:0000375 - RNA splicing, via transesterification reactions

GO:0000398 - mRNA splicing, via spliceosome

GO:0009966 - regulation of signal transduction

GO:0016310 - phosphorylation

GO:0036211 - protein modification process

GO:0032946 - positive regulation of mononuclear cell proliferation

GO:0043067 - regulation of programmed cell death

GO:0048584 - positive regulation of response to stimulus

GO:0043067 - regulation of programmed cell death

GO:0006355 - regulation of transcription, DNA-dependent

GO:0019219 - regulation of nucleobasecontaining compound metabolic process

GO:0010638 - positive regulation of organelle organization

GO:0046651 - lymphocyte proliferation

GO:0032845 - negative regulation of homeostatic process

${ }^{(*)}$ No enrichment for GO terms. 


\subsection{Discussion}

The development of new and more powerful computational resources in the last years has fuelled a surge of bioinformatics methods that have enabled researchers to analyse data sets of increasing complexity. As a result, systems biology has flourished and has provided valuable insight into the dynamic and complex nature of the immune system.

One of the basic principles underlying this holistic approach is the perception that the whole of living organisms are more than the sum of their parts. In the context of genomics, this aphorism translates into the observation that the understanding of cellular programs is much more complex than the identification of individual genes implicated in any given pathway, it requires the study of the network of relationships established at the molecular and cellular levels.

In this respect, correlation networks tools have proved to be much more useful than classical statistical methods to analyse large, high-dimensional data sets. ${ }^{(299)}$ WGCNA, in particular, has been successfully employed to analyse gene expression data in various contexts, i.e. brain cancer, ${ }^{(231)}$ yeast cell cycle,${ }^{(300)}$ mouse genetics, ${ }^{(301-304)}$ primate brain tissue, ${ }^{(305-307)}$ diabetes, ${ }^{(308)}$ chronic fatigue patients ${ }^{(309)}$ and plants. ${ }^{(310)}$ There is now ample evidence that gene correlation identifies clusters of co-regulated or co-expressed genes that frequently act as members of a biological pathway. For instance, this methodology was instrumental in the evolution of our understanding of 
macrophage differentiation extending the concept of macrophage activation from $\mathrm{M} 1$ versus $\mathrm{M} 2$ polarisation to a spectrum model, characterized by a variety of programs linked to chronic inflammation granulomatous diseases. ${ }^{(311)}$

By using WGCNA to explore the MataHari T cells data set, and assemble a signed network of highly correlated genes across the different study groups and tissues, 31 modules of positively correlated genes were identified (Figure 35 and Table 13). These groups of genes were shown to have distinct patterns of expression depending on the site from which samples were collected (Figure 37) and represented a robust network of genes involved in GVHD pathology, as the majority of them were strongly preserved in an independent murine GVHD model data set (Figure 39).

Moreover, the modules with the strongest preservation statistics $\left(Z_{\text {summary }} \geq 10\right)$ were shown to accurately distinguish between blood $\mathrm{CD}^{+} \mathrm{T}$ cell samples from alloBMT or synBMT recipients, and more importantly, to segregate those with GVHD, both in a non-human primate model of GVHD and with patient samples (Figure 40 - Figure 42). The paucity of published data regarding donor $\mathrm{T}$ cell gene expression at the sites of tissue injury led to the development of a collaborative study with the Newcastle University Institute of Cellular Medicine aimed at characterising the differences in $\mathrm{CD}^{+} \mathrm{T}$ cell effector programs isolated from the blood vs the skin of transplanted patients with GVHD. Even though at this time only a limited number of samples were available for analysis, the results obtained with them support the efficacy of this modular organisation in identifying tissue-specific groups of genes overrepresented in GVHD target organs (Figure 43). Nevertheless, further 
validation of the GVHD target organs-associated modules is required, in particular for modules associated with gastrointestinal pathology.

The characterisation of these highly preserved modules (Table 14, Apendix - Supplementary Table 3) provided further evidence supporting the biological relevance of the diversity of donor $\mathrm{CD}^{+} \mathrm{T}_{\text {eff }}$ cells' transcriptional profiles during the development of GVHD. The SLO-associated modules were found to mainly represent cellular programs linked with priming and proliferation, including cardinal pathways of T cell activation (e.g. TLR and RIG-I signalling), oxidative metabolic activity (e.g. fatty acid oxidation), and cell cycle regulation (e.g. CDK1 and aurora kinase pathways). The GVHD target organs-associated modules, on the other hand, were mostly related to effector function related pathways, highlighting their role in determining target organ damage. Interestingly, these modules seem to define a stepwise differentiation program which comprises a nuclear set of instructions linked to early Tc1/Tc17 polarisation, common to all GVHD target organs, that are further enhanced by tissue-specific cellular programs.

These observations suggest that the peripheral tissues play autonomous and dominant roles in determining $T_{\text {eff }}$ cell function. Both in the gut and in the skin, a progression is observed in their subcompartments, from cellular programs centred on migration in response to chemokine signalling, in the lamina propria and dermis, to programs centred on cytotoxic function, in the intra-epithelial compartment and epidermis.

Interestingly, this difference in subcompartments is most striking in the skin, where a large number of genes encoding multiple pro-inflammatory 
cytokines (i.e. Ifng, I/2, I/3, I/13, I/17a, Csf1, Csf2) and cytokine receptors (e.g. I/2ra, I/1r1) are highly correlated with $\mathrm{T}_{\text {eff }}$ cells in the epidermis, as part of module 28 .

One possible explanation for this phenomenon could be that as the $\mathrm{T}_{\text {eff }}$ cells migrate through the tissues they receive environmental cues which shape their post-activation differentiation path. Such a model would imply the requirement of in situ interactions between the donor $\mathrm{CD} 8^{+} \mathrm{T}$ cells and tissue resident cell populations which would "license" them to achieve their full effector potential. 


\section{Donor $\mathrm{CD}^{+} \mathrm{T}_{\text {eff }}$ pathogenicity in the skin is regulated in situ by epidermal Langerhans cells}

To better understand the cellular mechanisms underlying the divergence of transcriptional profiles among the GVHD target organs, and in particular between subcompartments within each organ, the remainder of the study was focused on the skin.

The skin's immune niche is composed of two main compartments: the dermis, populated by macrophages, dendritic cells, mast cells, $ү \delta \bar{T}$ cells and $\alpha \beta$ T cells; and the epidermis, where Langerhans cells (LC), dendritic epidermal y $\delta \mathrm{T}$ cells (DETC) and tissue memory $C D 8^{+} \mathrm{T}$ cells $\left(\mathrm{T}_{\mathrm{RM}}\right)$ are the only resident immune cell populations. ${ }^{(312,313)}$ The maintenance of this cellular network and its tight regulation are crucial for an effective cutaneous immune surveillance, as made evident from the increased incidence and severity of cutaneous infections and malignancies in immunocompromised patients, and by the development of a variety of skin disorders when the skin defence mechanisms are misdirected. ${ }^{(314)}$

In the context of alloBMT, the skin's microenvironment is greatly disrupted. As a consequence of the conditioning regimen, most of the host skin immune populations are depleted and quickly replaced by donor derived ones. Notably, the Langerhans cells are the exception: being radioresistant and 
capable of proliferating locally and maintaining homeostasis independently of the bone marrow, Langerhans cells persist long after BMT and full donor chimerism is only achieved in the presence of donor T cells. ${ }^{(315-317)}$

The characterisation of the changes in the skin immune network following alloBMT has been one of the focus of $\mathrm{Dr}$ Bennett and Prof Chakraverty's lab. Studies conducted by Dr Thomas Conlan, Dr Cara Lomas and Dr Ivana Ferrer in the minor histocompatibility antigen mismatched model of acute GVHD (MataHari T cell model) have shown that skin infiltrating donor $\mathrm{CD}^{+} \mathrm{T}$ cells migrate through the dermis and invade the epidermis, localising preferentially along the basement membrane, in close proximity to the Langerhans cells (Figure 44).

The analysis of the donor $\mathrm{CD}^{+} \mathrm{T}$ cells epidermal infiltration and of the Langerhans cells replacement kinetics revealed that even though the ratio between host and donor LC is reversed in the first 3 weeks following donor CD8+ $T$ cell entrance into the skin, a considerable number of host derived LC still populate the epidermis at that point (Figure 45).

Moreover, Dr Thomas Conlan's work in the Langerin-DTR system showed a unique association between LC and skin GVHD development. ${ }^{(217)}$ Expressing the simian diphtheria toxin (DT) receptor downstream of the Langerin gene promotor, these transgenic mice become selectively depleted of epidermal LC after treatment with DT. Using the Langerin-DTR mice as recipients for alloBMT, Dr Conlan was able to establish that in the absence of LC the severity of cutaneous GVHD was greatly abated, which was directly correlated with a significant reduction in the number of donor $\mathrm{CD}^{+} \mathrm{T}$ cells 
infiltrating the epidermis (Figure 46). This effect was observed both after systemic or localised LC depletion (Figure 47), suggesting that GVHD immunopathology modulation by LC occurred in situ. 
A

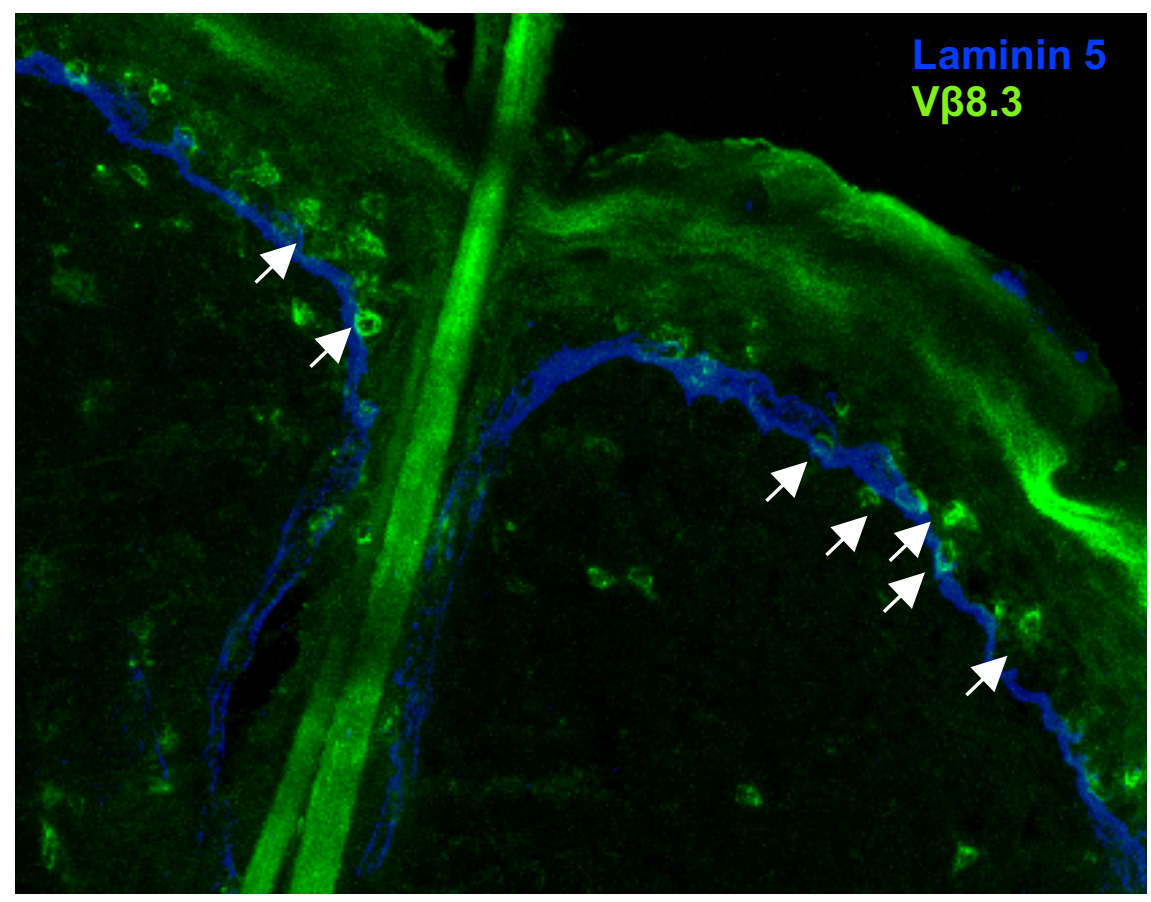

B

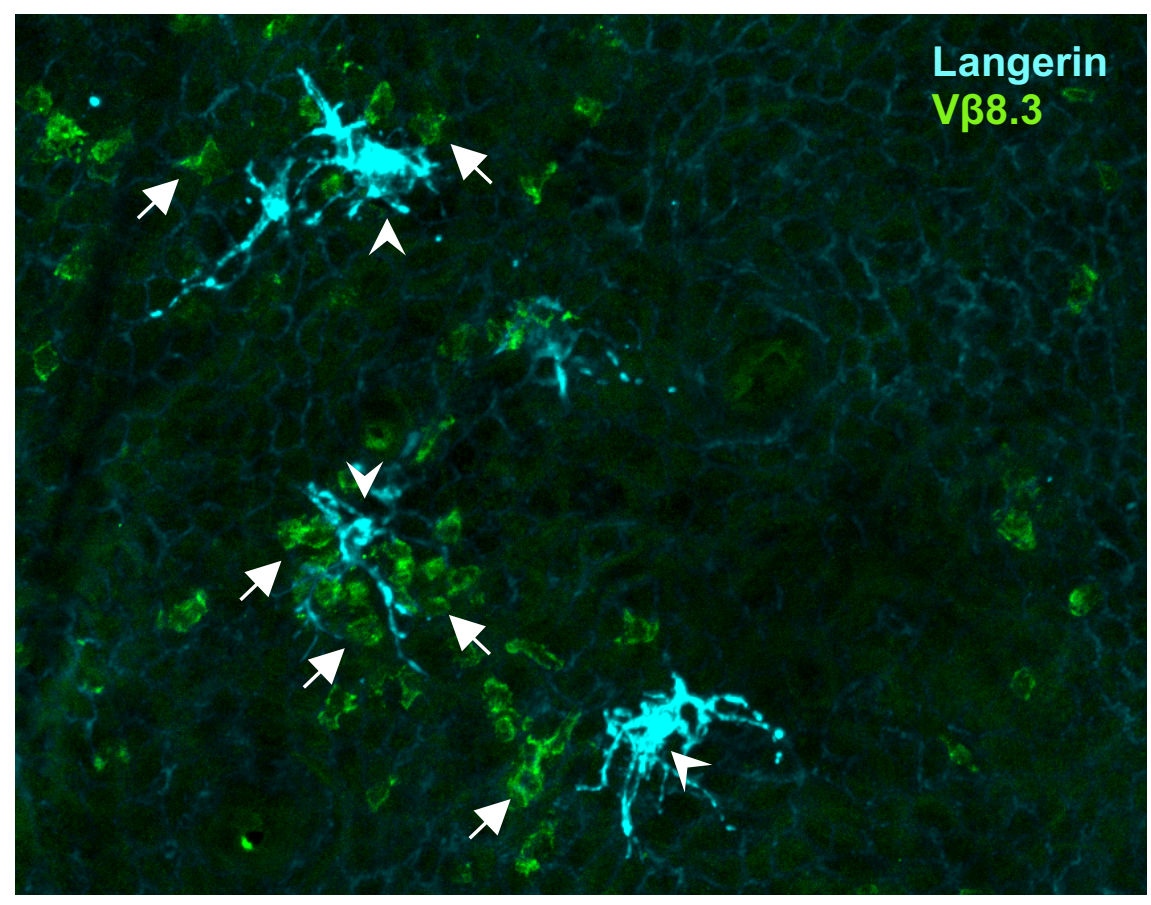

Figure 44. Immunofluorescence staining of the skin of alloBMT recipients with cutaneous acute GVHD (MataHari T cell model). (A) Cross section of the dermal-epidermal transition showing the preferential localisation of donor $\mathrm{CD}^{+} \mathrm{T}$ cells (green; arrows) along the basement membrane (blue). (B) Epidermal sheet showing the close proximity of donor $\mathrm{CD}^{+} \mathrm{T}$ cells (green; arrows) and LC (cyan; arrow heads).

Courtesy of Dr. Cara Lomas and Dr Ivana Ferrer. 
A

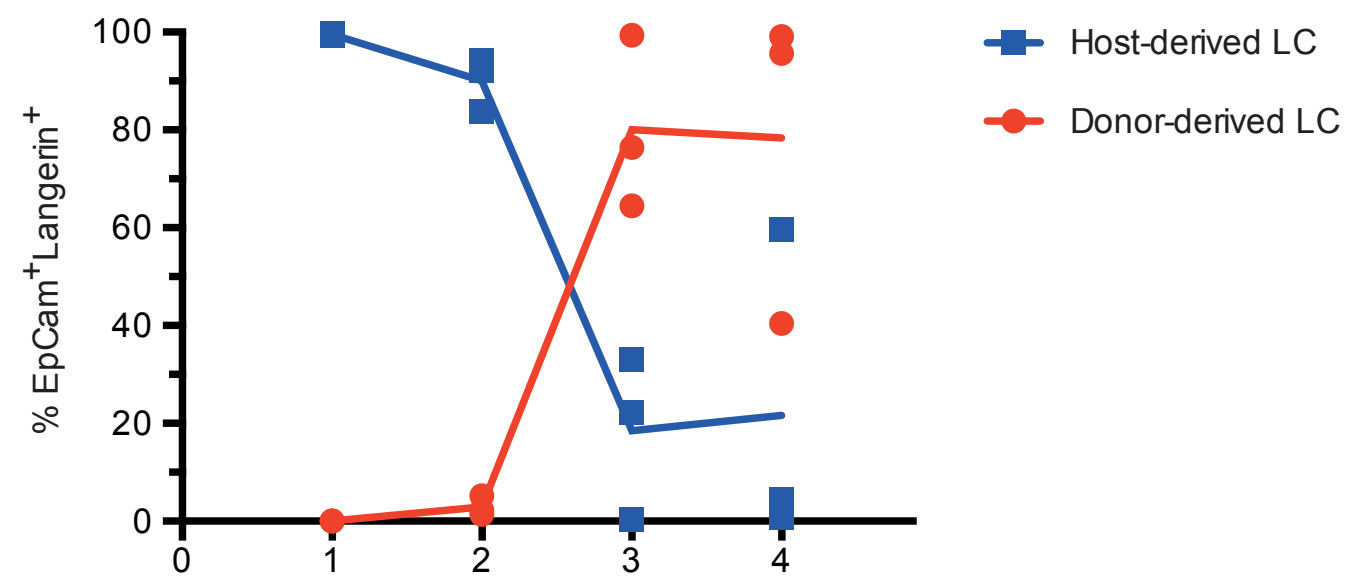

Weeks

B

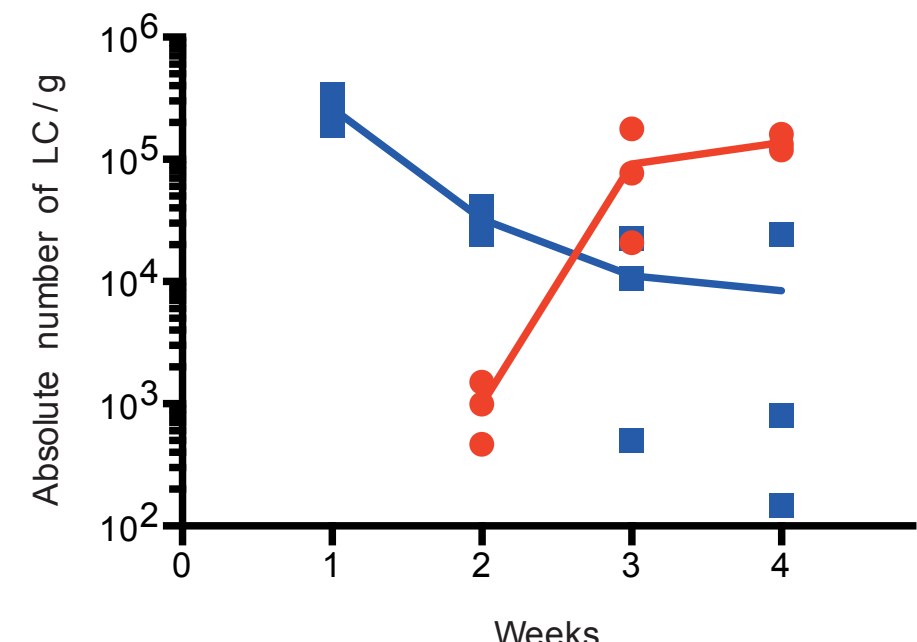

Figure 45. Host-derived LC replacement kinetics after alloBMT. Although the ratio between host and donor LC is reversed in the first 3 weeks following donor CD8+ T cell entrance into the skin (A), a considerable number of host-derived LC still populate the epidermis at that point (B).

Unpublished data, courtesy of Dr Ivana Ferrer. 
A

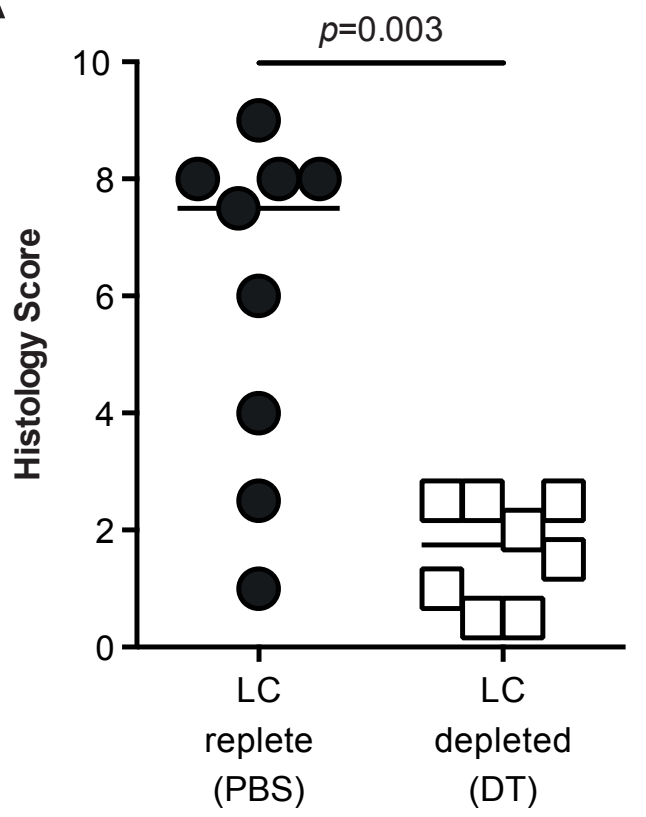

B

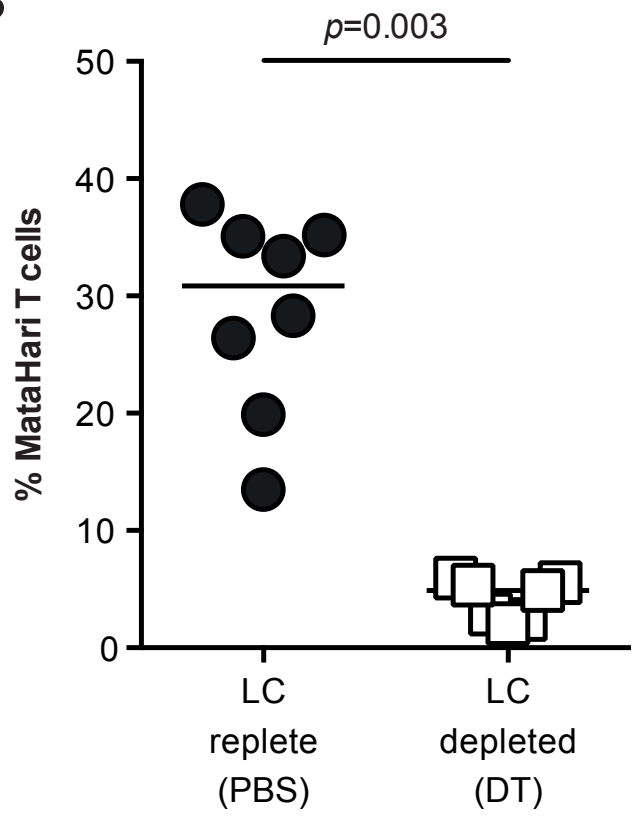

Figure 46. Effect of systemic host LC depletion on cutaneous acute GVHD. Comparison between the histology scores $(\mathbf{A})$ and donor T cell infiltration of the epidermis (B) of LC replete (black circles) and LC depleted (white squares) alloBMT recipients. In the absence of LC, the severity of cutaneous GVHD is reduced, which was accompanied by a significant contraction in the number of epidermal donor T cells (PBS treated: $n=9$; DT treated: $n=8$ ).

Courtesy of Dr Thomas Conlan.

A

\section{PBS control}

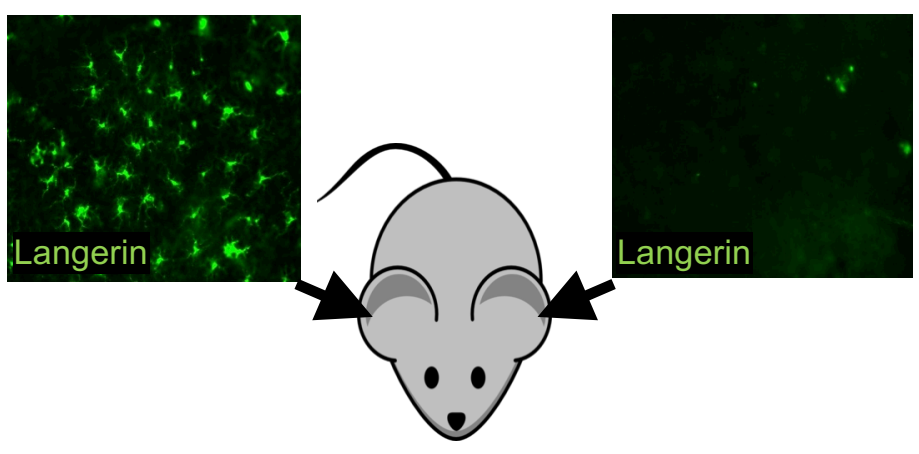

Langerin.DTR
B

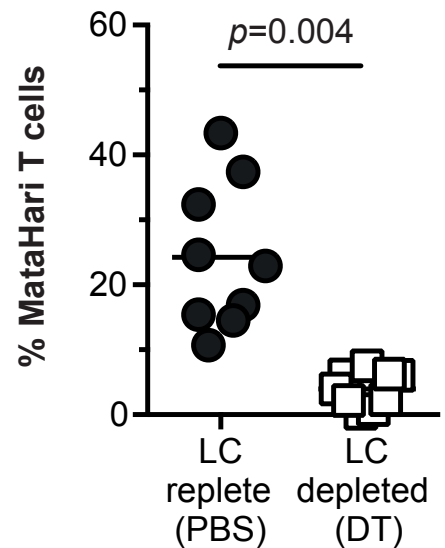

Figure 47. Effect of localised host LC depletion on donor $\mathrm{CD}^{+} \mathrm{T}$ cell infiltration of the epidermis.

(A) Langerin-DTR alloBMT recipients received PBS to the right ear and DT to the left ear by intradermal injections at D-21. (B) At D+7 post-transplant, accummulation of donor $C D 8^{+} \mathrm{T}_{\text {eff }}$ cells was significantly reduced in the ear that had been depleted of $L C$ ( $n=9$ in each group).

Courtesy of Dr Thomas Conlan. 


\subsection{LC depletion reduces the differences between dermal and epidermal donor $\mathrm{CD}^{+} \mathrm{T}_{\text {eff }}$ cells transcriptional profiles}

To further investigate the role of host LC in the development of GVHD, the adapted MataHari T cell $\rightarrow$ Langerin-DTR model was used, in which MataHari CD8 ${ }^{+}$T cells were transferred together with female wild-type C57BL/6 bone marrow and polyclonal $\mathrm{CD}^{+} \mathrm{T}$ cells into lethally irradiated Langerin-DTR male recipients, treated either with PBS or DT prior to the transplant. At D+7 post-transplant, the donor derived $\mathrm{CD}^{+} \mathrm{T}$ cells were isolated from the peripheral lymph nodes, dermis and epidermis of the recipients; the samples' RNA was purified and a microarray analysis was performed using the Affymetrix GeneChip ${ }^{\circledR}$ Mouse Gene 2.0 ST array, as previously described.

As an exploratory analysis of the dataset, the effect of LC depletion was assessed by performing pairwise comparisons of the transcriptional profiles of the six groups of samples. As illustrated by the correlation matrix depicted in Figure 48, DT treatment had no significant effect on the lymph nodes or on the dermis, but induced a change in the gene expression of $\mathrm{T}_{\text {eff }}$ cells which infiltrate the epidermis, reducing the disparity between the two skin compartments. This observation is consistent with the knowledge that treatment with DT is nontoxic to mice and only affects cells expressing the simian DT receptor. 


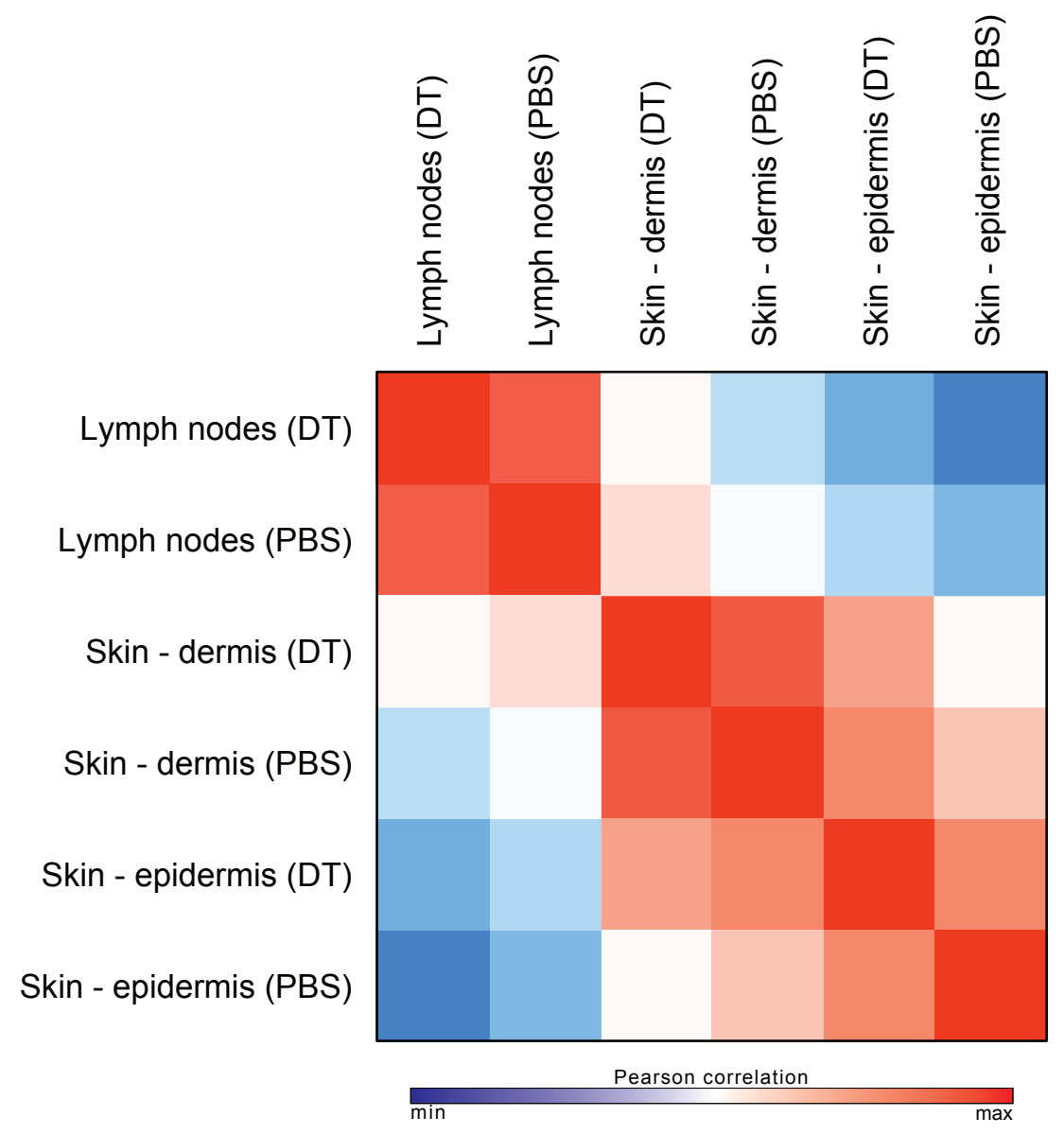

Figure 48. Correlation matrix comparing the transcription profiles of donor $C D 8^{+} \mathrm{T}_{\text {eff }}$ cells isolated from the lymph nodes, dermis and epidermis of $L C$ depleted (DT) and LC replete (PBS) alloBMT recipients, at D+7 post-tranplant. Groups represent the average expression of the biological replicates. The colour code reflects the Pearson correlation coefficient for each comparison. LC depletion affects mainly the transcritional profile of epidermal T cells, reducing the differences between the two skin compartments. 


\subsection{In the absence of LC, epidermal donor T cells fail to fully up-regulate the effector cell programs}

In order to better characterise the changes induced by DT treatment, the enrichment for the gene sets defined in the KEGG PATHWAY collection was determined. By comparing dermis vs lymph nodes and epidermis vs dermis through GSEA, it was possible to identify the shifts in cellular programs as cells transited from one compartment to another, and to evaluate the impact that LC depletion produced on these processes (Appendix - Supplementary Table 4). In Figure 49 the normalised enrichment score (NES) for the 91 gene sets (rows) significantly over- or under-represented in each pair of tissues were compared according to treatment (columns). The results confirmed that DT treatment had a negligible effect on the array of pathways that were up- and down-regulated when donor $\mathrm{CD} 8^{+} \mathrm{T}$ cells migrated from the lymph nodes to the dermis, and evidenced that a variety of cellular programs that are normally triggered upon transition from the dermis to epidermis failed to be activated in the absence of LC.

Examination of the gene sets that were differentially enriched in the epidermis in the presence of LC revealed that the genetic programs were related to: cellular processes (such as cell growth, death, transport and catabolism), signal transduction, genetic information processing (mainly replication and repair, transcription and translation), energy and nucleic acid 
metabolism; and were linked to immune system function and immune disease, namely GVHD (Figure 50, Appendix - Supplementary Table 4). 


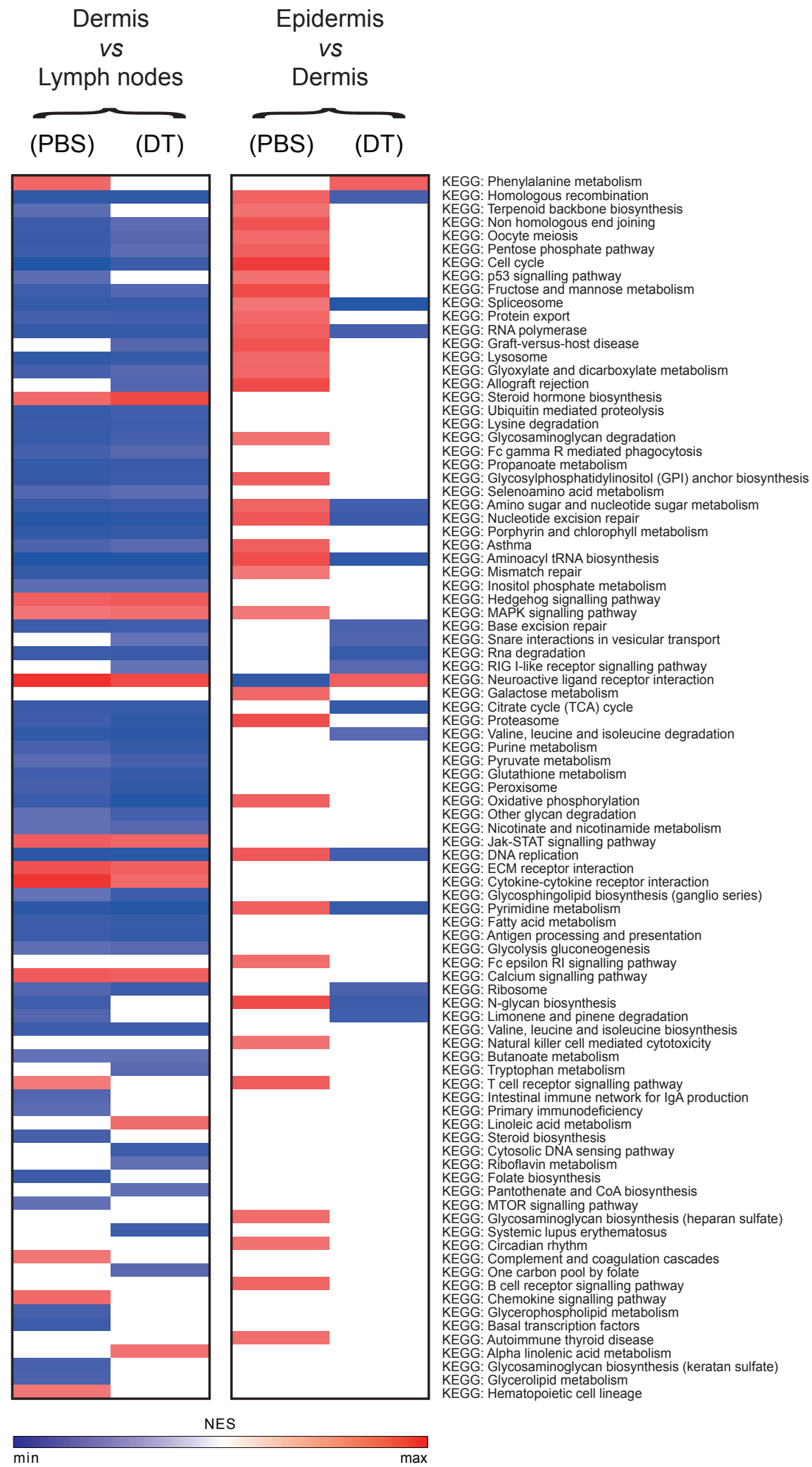

Figure 49. Heat map showing the enrichment for the 91 gene sets significantly over- or under-represented in the dermis vs the lymph nodes and in the epidermis vs the dermis, with or without LC. The colour code reflects the normalised enrichment score (NES) for each gene set. Note that the majority of pathways up-regulated by $T$ cells in a LC replete epidermis are absent when LC have been depleted. 


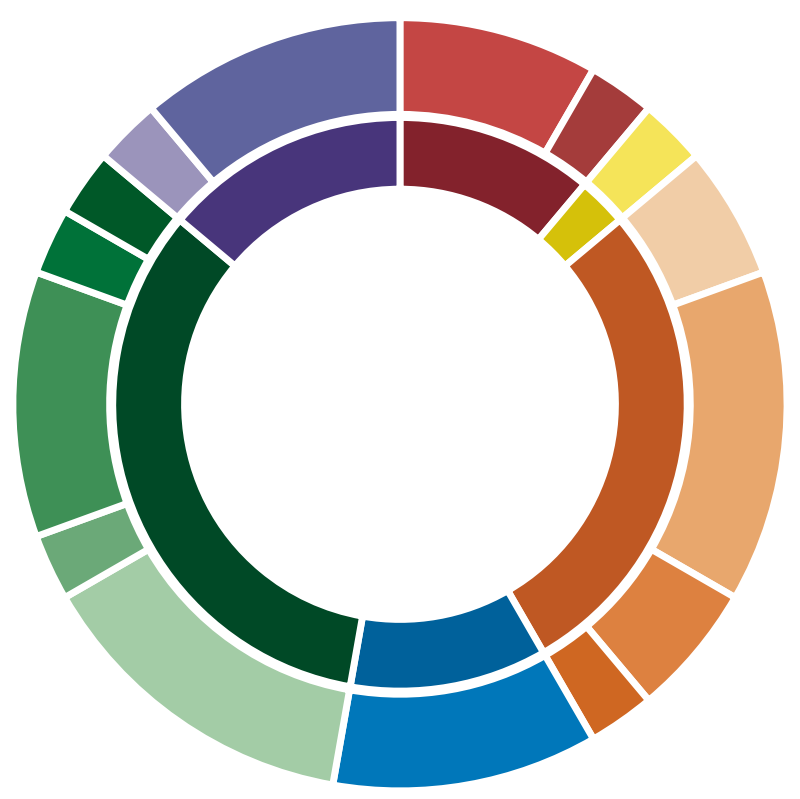

1st level classification (inner circle)

Cellular Processes

Environmental Information Processing

Genetic Information Processing

Human Diseases

Metabolism

Organismal Systems 2nd level classification (outer circle)

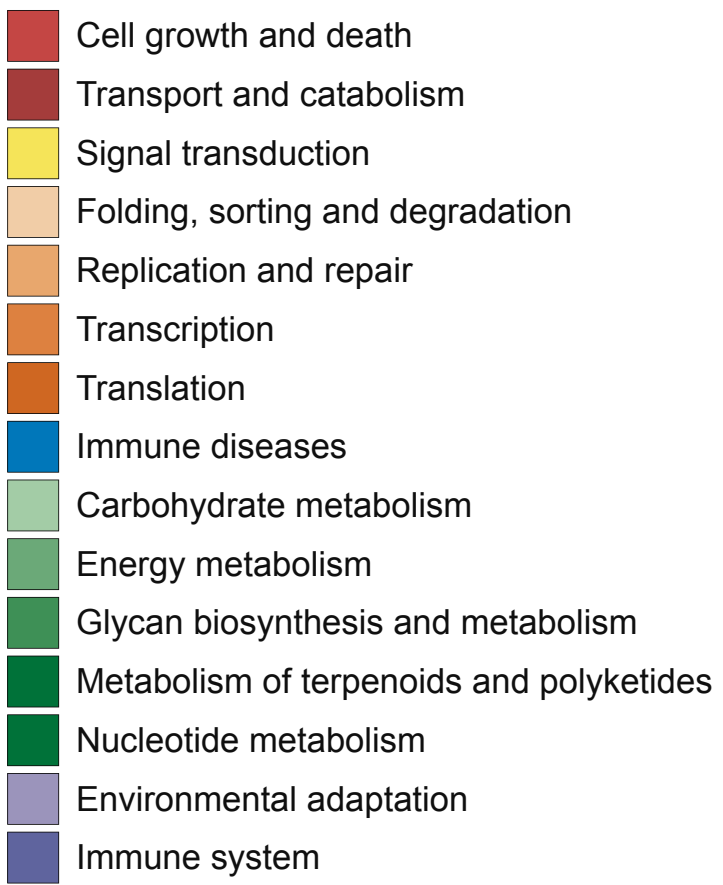

Figure 50. Classification of the gene sets that were differentially enriched in the epidermis in the presence of LC according to KEGG PATHWAY mapping. 


\subsection{Donor $\mathrm{CD}^{+} \mathrm{T}$ cells expression of the epidermal specific transcriptional signature is regulated by host LC}

To test the concept that LC were implicated in defining an epidermal specific transcriptional signature, the expression pattern of the genes from module 28 , previously identified by WGCNA in the MataHari T cell model as being highly correlated with the epidermis, was compared between PBS and DT treated groups. As depicted in the heat map in Figure 51, the 443 genes that form this module were consistently highly expressed in the LC replete epidermis samples (PBS treated), however, in the absence of LC (DT treated), this pattern was broadly disrupted and only small clusters of genes were expressed to the same extent as in the controls. This reduction in the level of expression was most profound for the module's hub genes (Figure 52) denoting their central role in driving the expression of the remaining genes.

The analysis of the overrepresentation of the GO categories and KEGG pathways for the set of module 28 genes that failed to be upregulated in the epidermis in the DT treated group (Appendix - Supplementary Figure $\mathbf{4 0}$ and Supplementary Table 5) indicated that the main biological processes affected by the absence of LC were related to regulation of leukocyte proliferation / apoptosis and development of the immune response, involving cytokine-cytokine receptor interactions, and signalling through Jak-STAT, MAPK, TLR and TCR pathways. 


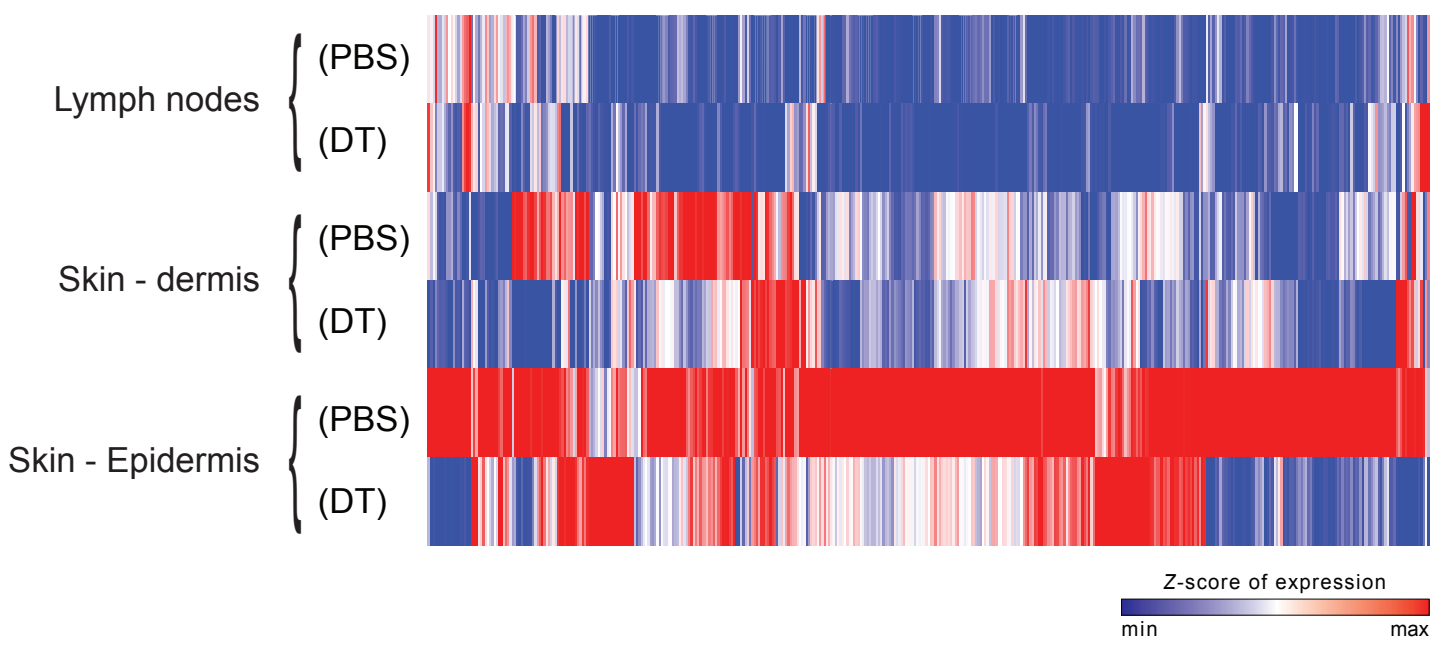

Figure 51. Heat map of the 443 genes that form module 28, comparing the transcriptional profiles of the lymph nodes, dermis and epidermis, in the presence (PBS) or absence (DT) of LC. The colour code reflects the Z-score of expression. LC depletion leads to failure in T cell upregulation of this epidermal-specific gene signature.

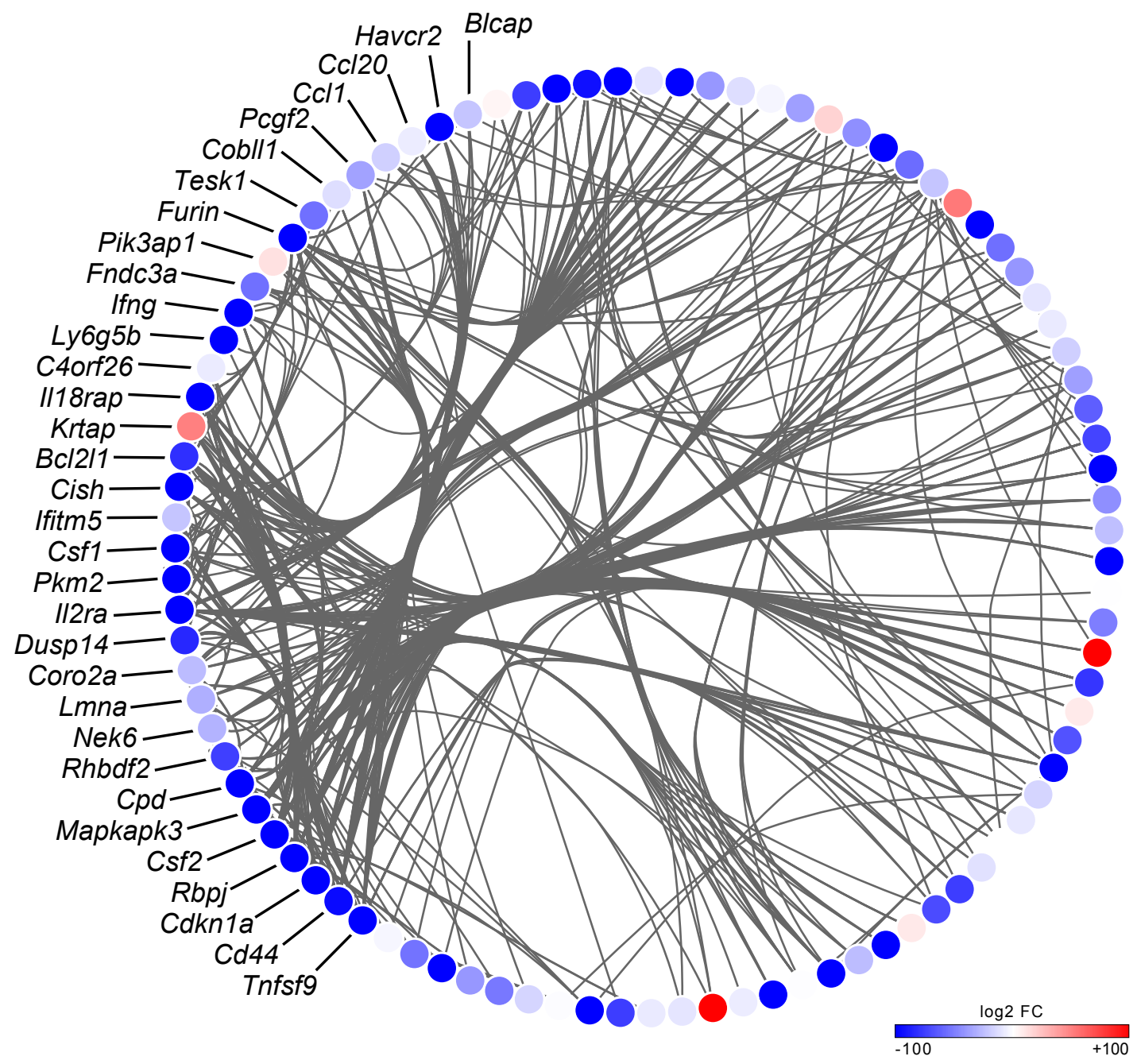

Figure 52. Module 28 top 100 gene network, where the circles represent the genes and the lines represent the connectivity between the genes. The colour code reflects the log2 fold change (log2 FC) in expression level in the epidermis of PBS treated vs DT treated groups. Note the extreme down-regulation of most of module's hub genes in the absence of LC. 


\subsection{LC dictate donor $\mathrm{T}$ cell accumulation, survival, effector} function and $T_{R M}$ differentiation potential in the epidermis

To validate these results, a second set of experiments in the MataHari T cell $\rightarrow$ Langerin-DTR model was conducted to evaluate by multicolour flow cytometry the proliferation, effector function and survival of skin infiltrating donor $\mathrm{CD}^{+} \mathrm{T}$ cells in the presence or absence of LC. AlloBMT recipients treated with either PBS or DT at D-21 were euthanized at D+7 and samples from the lymph nodes, dermis and epidermis were collected (Figure 53). The proliferative index of donor $\mathrm{CD} 8^{+} \mathrm{T}$ cells was determined by EdU staining (alloBMT recipients were given EdU $25 \mathrm{mg} / \mathrm{kg}$ iv $12 \mathrm{~h}$ prior to sacrifice); survival was assessed by quantification of caspase-3 activity; ex vivo cytotoxic effector function was measured by staining for IFN-y production, without further re-stimulation (Figure 54 and Figure 55).

Although no difference was detected on the proliferation index of epidermal T cells (Figure 54-A and Figure 55-A), it was invariably found that a higher proportion of these cells were apoptotic (PBS: $5.9 \% \pm 0.7$ vs DT: $7.8 \%$ \pm 0.4 ; mean $\pm \mathrm{SD}$ ) (Figure 54-B and Figure 55-B). Moreover, in the absence of LC, $T_{\text {eff }}$ cells produced significantly lower amounts of IFN-Y (PBS: $20.2 \% \pm$ 5.6 vs DT: $7.2 \% \pm 3.6$; mean \pm SD) (Figure $54-C$ and Figure 55-C).

To investigate the long term effects of host LC depletion on donor CD8 ${ }^{+}$ $T_{\text {eff }}$ cells' capacity to differentiate into tissue-resident memory $T$ cells $\left(T_{R M}\right)$, a 
long term experiment was conducted in collaboration with Dr Ivana Ferrer and Ms Sophie Ward in which PBS or DT treated transplanted Langerin-DTR mice (MataHari T cell $\rightarrow$ Langerin-DTR model) were sacrificed at weeks 1, 2, 3 and 4 post-transplant and skin samples collected for study of the epidermal kinetics of donor derived $\mathrm{CD}^{+} \mathrm{T}_{\mathrm{RM}}$ cells based on CD69 and CD103 expression (Figure 56). Interestingly, the results showed that in the absence of host LC the accumulation of donor $\mathrm{CD}^{+} \mathrm{T}_{\mathrm{RM}}$ cells in the epidermis was impaired, an effect that gained momentum with time and by week 4 post-transplant, the difference between the percentage of epidermal $\mathrm{CD} 69^{+} \mathrm{CD} 103^{+}$MataHari T cells in the two groups achieved statistical significance (PBS: $49.9 \% \pm 9.9$ vs DT: $37 \% \pm$ 10; mean \pm SD) (Figure 57). 


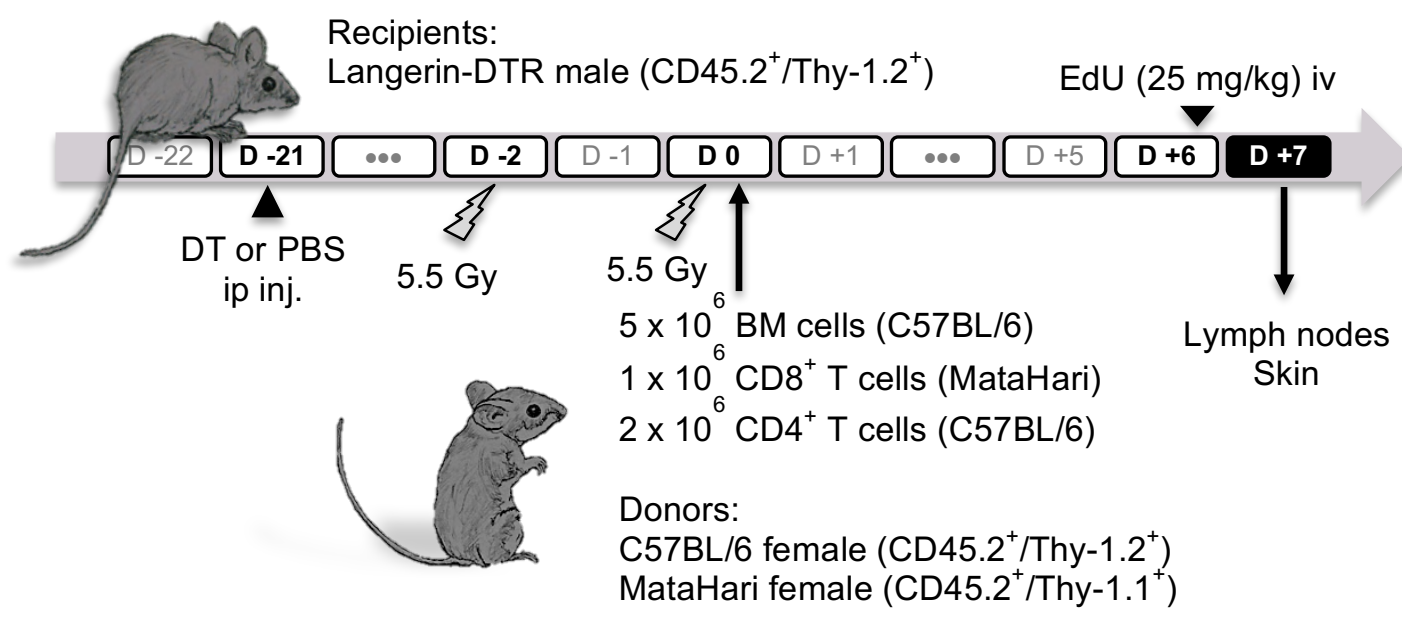

Figure 53. Experimental design to evaluate the effect of LC depletion on proliferation, effector function and survival of skin infiltrating donor $\mathrm{CD} 8^{+} \mathrm{T}$ cells. 


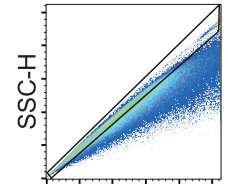

SSC-A
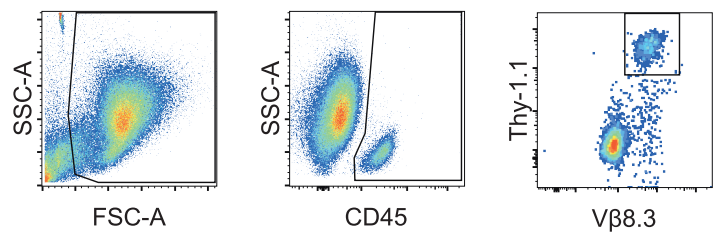

Lymph nodes

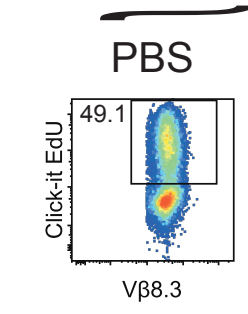

Vß8.3

DT

A

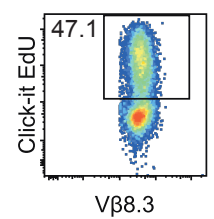

B

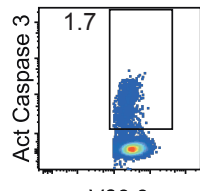

Vß8.3
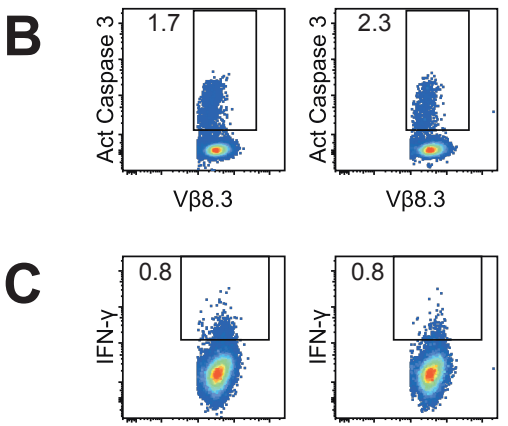

V $\beta 8.3$

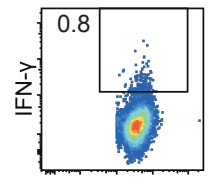

VB8.3

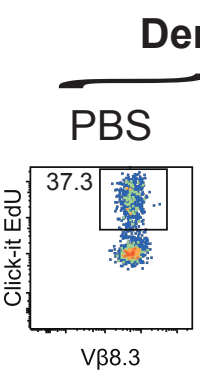

Dermis
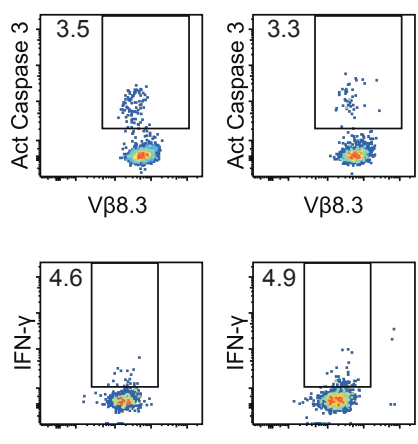

V 88.3

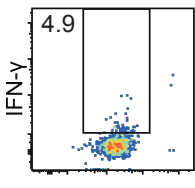

VB8.3

\section{Epidermis}

PBS

DT
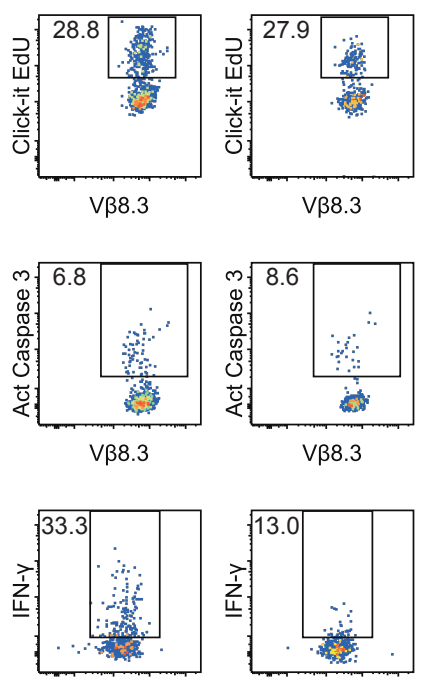

VB8.3

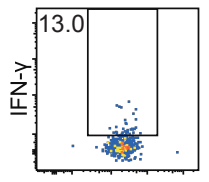

VB8.3

Figure 54. Assessment of the proliferation (A), survival (B) and effector function (C) of donor $\mathrm{CD}^{+} \mathrm{T}$ cells by FACS staining. Samples from the lymph nodes, dermis and epidermis were stained for EdU incorporation, active caspase-3 and IFN-y. The gating strategy used to identify donor $\mathrm{CD}^{+} \mathrm{T}$ cells is shown, as well as representative plots for each group and condition. 
A

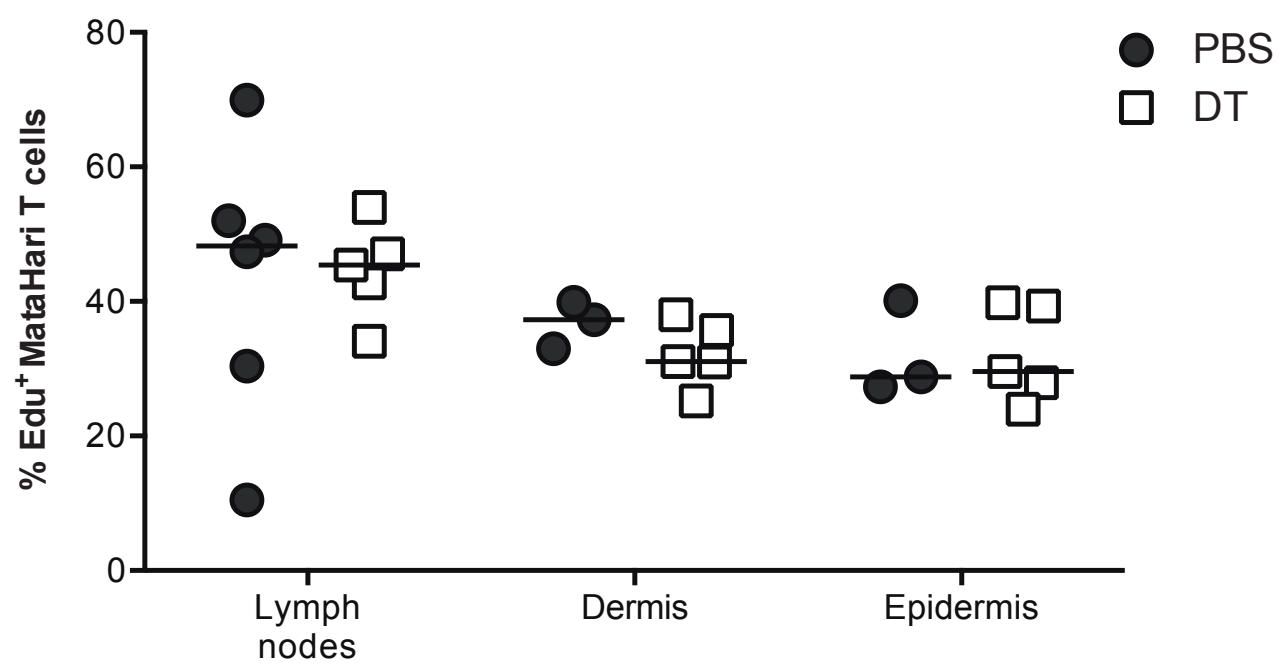

B

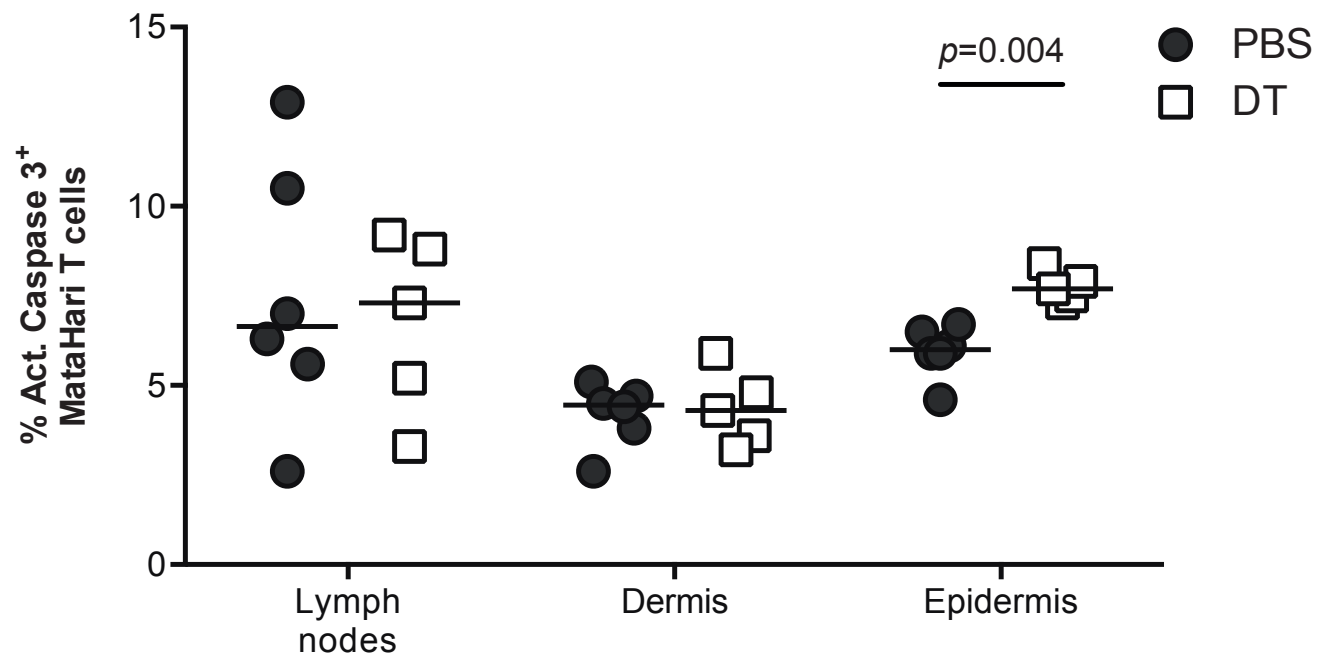

C

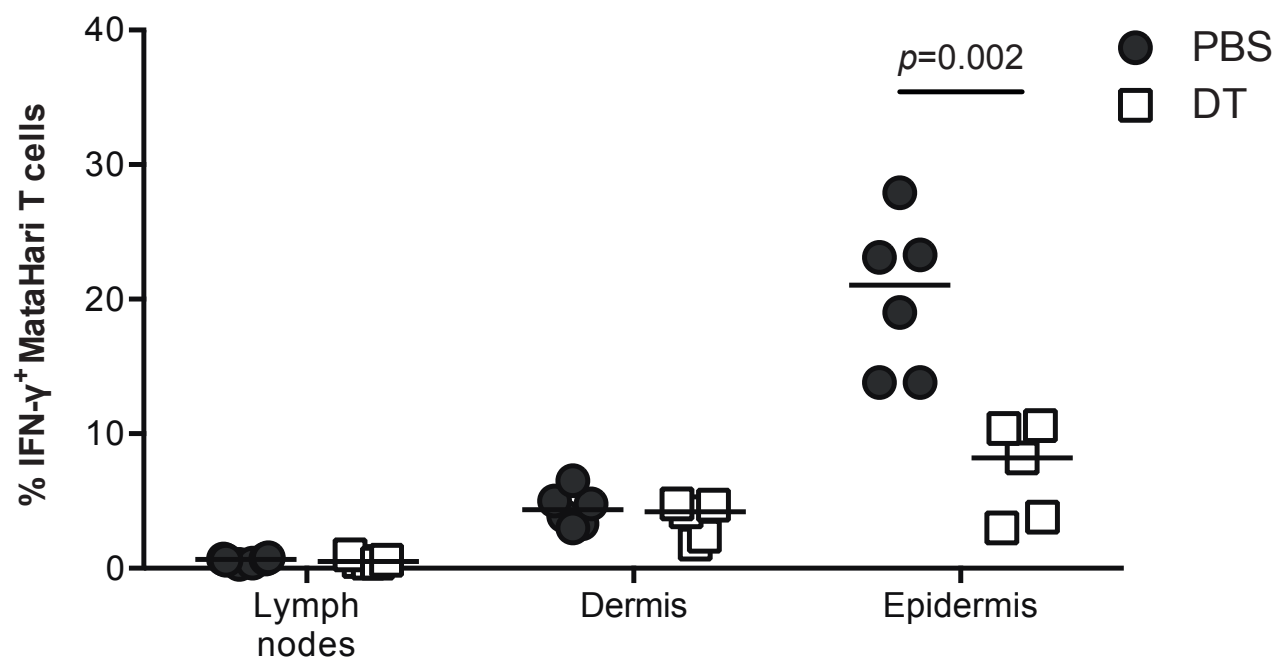

Figure 55. Summary data referring to the study of the effect of LC depletion upon proliferation (A), survival (B) and effector function (C) of donor $C D 8^{+} T_{\text {eff }}$ cells. Although no difference in proliferation was observed between groups, in the absence of LC, the percentage of donor $\mathrm{CD}^{+} \mathrm{T}_{\text {eff }}$ cells was consistently higher and the production of INF-y was significantly reduced. PBS: $n=6$; DT: $n=5$ (except for EdU staining of dermis and epidermis where PBS: $n=3$ ); data pooled from 2 independent experiments. 


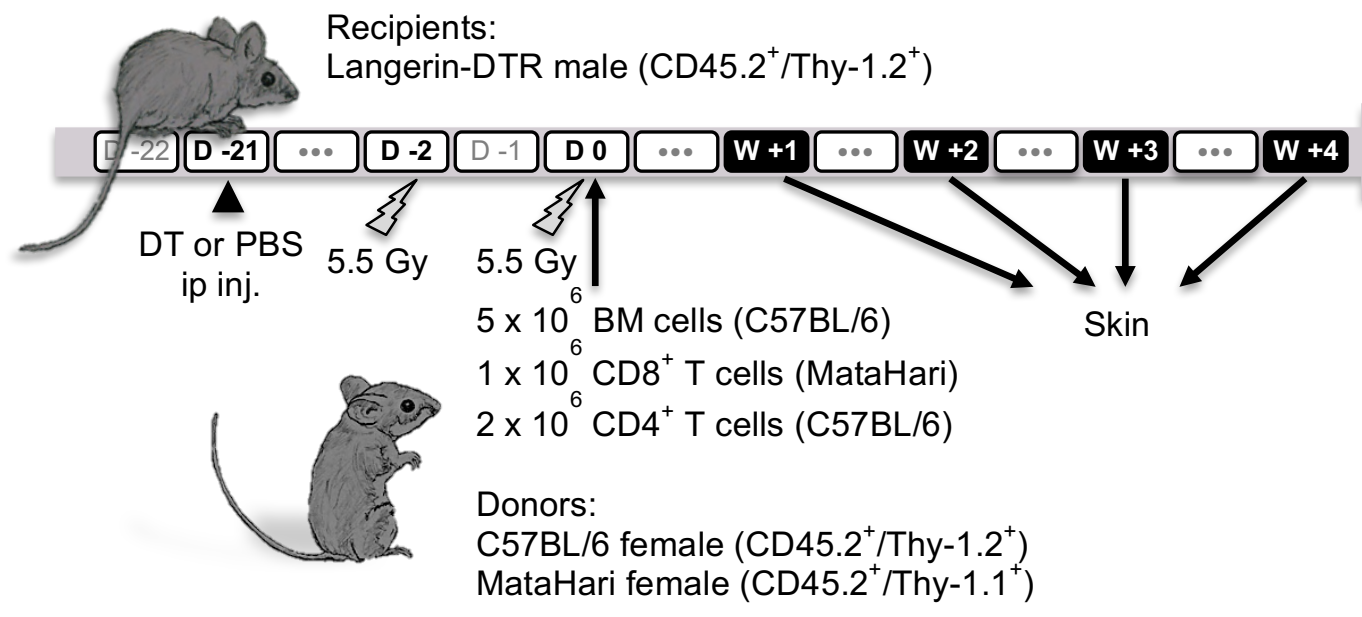

Figure 56. Experimental design to evaluate the effect of $\mathrm{LC}$ depletion donor $\mathrm{CD} 8^{+} \mathrm{T}$ cells capacity to differentiate into epidermal $\mathrm{T}_{\mathrm{RM}}$ cells. 
A

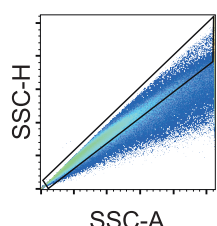

SSC-A

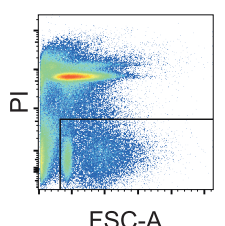

Week +2
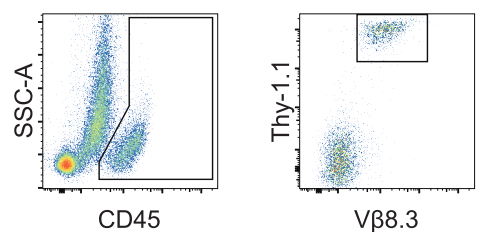

\section{Week +1}

PBS

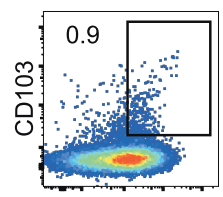

DT
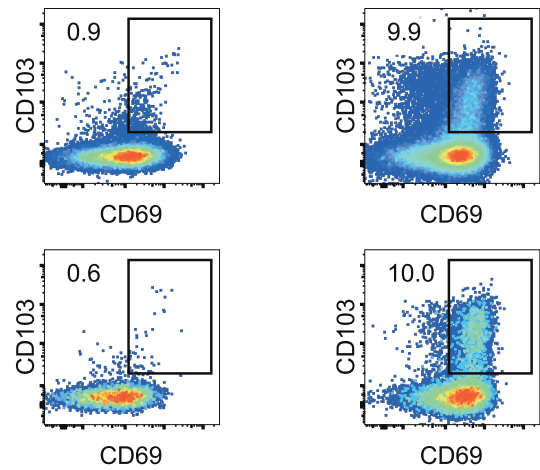

CD69

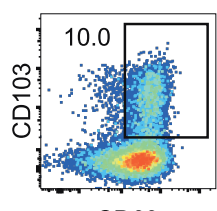

CD69

Week +3

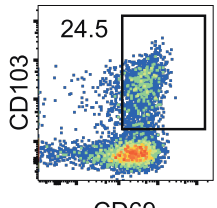

CD69

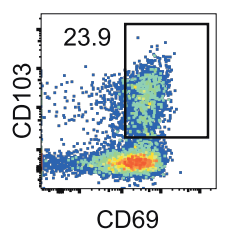

Week +4
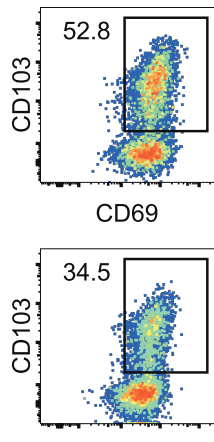

CD69

B

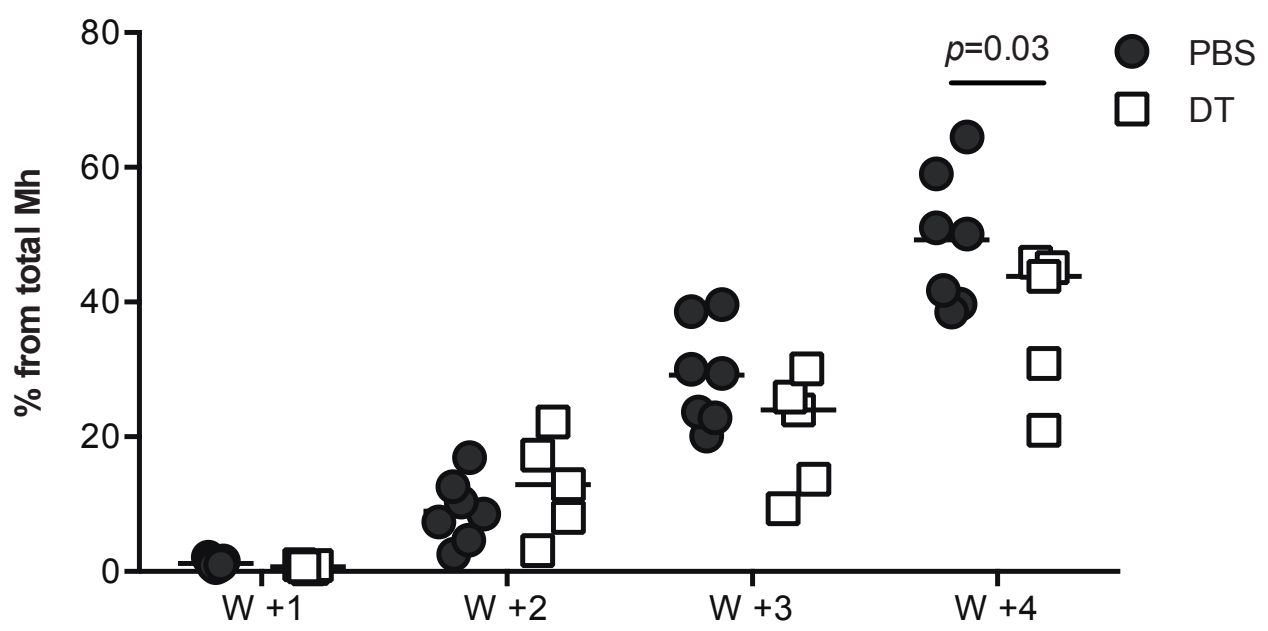

Figure 57. Kinetics of donor derived $C D 8^{+}$epidermal $T_{R M}$ cells in the presence or absence of LC. (A) Gating strategy used to identify donor $\mathrm{CD}^{+} \mathrm{T}$ cells in the epidermis. Representative plots of CD103 and CD69 staining are shown for each time point and condition. (B) Summary data. In the absence of $L C$, a lower proportion of donor $T$ cells express a $T_{R M}$ cell phenotype. For each time point, PBS: $n=7$, DT: $n=5$; data pooled from 2 independent experiments. 


\subsection{Responsiveness to IFN-Y by LC and Notch pathway signalling are central in the host $\mathrm{LC}$ - donor $\mathrm{T}$ cell crosstalk}

Altogether, the gene expression profiling and the protein expression data support the hypothesis that a crosstalk between LC and donor $\mathrm{CD}^{+} \mathrm{T}_{\text {eff }}$ cells occurs in the epidermis which regulates $\mathrm{T}$ cell effector function and fate, and ultimately determines the severity of the GVHD immune reaction. Consistent with this concept, in experiments performed by $\mathrm{Dr}$ Thomas Conlan which compared the transcription profile of LC after allogeneic versus syngeneic BMT controls, it was observed a significant enrichment for type I and type II IFN responsive genes (Table 15). ${ }^{(217)}$

One possible interpretation of these data is that a feed-forward loop involving $L C$ responsiveness to IFN- $y$ is required to be established for $L C$ to induce cognate activation, expansion and survival of incoming $\mathrm{T}$ cells. Moreover, in accordance with the current understanding of the relevance of Notch signalling to T cell alloreactivity, ${ }^{(318-320)}$ in particular to the production of IFN-y, ${ }^{(321)}$ the present work identified Rbpj and Furin, two key regulators of Notch pathway, as part of the group of genes that drives the expression of module 28 .

In order to test this hypothesis, two sets of experiments (Figure $\mathbf{5 8}$ and Figure 62) were performed using the MataHari T cell model that evaluated the 
effect of interrupting these two signalling pathways upon the accumulation, survival and IFN-y production of donor $\mathrm{CD}^{+} \mathrm{T}$ cells in the skin.

To evaluate the requirement of LC responsiveness to IFN- $\mathrm{y}$, Ifngr ${ }^{-/-}$or WT recipients were transplanted following the MataHari T cell model, with the exception that T cells were activated in vitro with concanavalin A and IL-7 prior to injection into the recipients, in order to overcome any priming deficit induced by absence of IFN-y receptor signalling in the SLO (Figure 58). At D+7 post-transplant samples from skin and spleen were collected and analysed by flow cytometry (Figure 59 - Figure 61).

Both the host $\mathrm{LC}$ and the donor $\mathrm{CD} 8^{+} \mathrm{T}_{\text {eff }}$ cells were analysed in this study. It was observed that although IFN-y signalling did not significantly affect LC expression levels of costimulatory molecules, such as CD40, CD70 or CD86, when unable to detect IFN-ץ signalling, LC failed to upregulate the expression of MHC class I H-2Db molecules (H-2Db MFI - WT: $1753 \pm 77$ vs Ifngr $1^{-1-}: 604 \pm 306$; mean $\pm \mathrm{SD}$ ) (Figure 59). As a result, the accumulation of donor $\mathrm{CD}^{+} \mathrm{T}_{\text {eff }}$ cells was reduced specifically in the epidermis of $/ f n g r 1^{-/-}$ recipients (WT: $20 \times 10^{3}$ cells $/ \mathrm{g} \pm 7 \times 10^{3}$ vs Ifngr $1^{-1-}: 4.7 \times 10^{3}$ cells $/ g \pm 4 \times 10^{3}$; mean $\pm S D$ ), even though the expansion of donor $C D 8^{+} T_{\text {eff }}$ cells in the spleen was similar in both groups and no difference in the absolute numbers of tissue infiltrating $T$ cells was noted in the dermis (Figure 60 and Figure 61-A). Remarkably, absence of IFN- $y$ receptor signalling in host LC neither increased T cell apoptosis (Figure 60 and Figure 61-B) nor reduced T cell production of IFN-y (Figure 60 and Figure 61-C). 
The impact of Notch signalling blockade on the development of cutaneous GVHD was assessed through the treatment of alloBMT recipients with LY411575, a potent inhibitor of the presenilin-dependent gamma secretase complex responsible for the release of the Notch Intracellular Domain (NICD) after ligand binding to the Notch receptor. For the purpose of minimising the interference with $T$ cell priming events, the treatment was given only from $D+5$ onwards, which corresponded to the onset of skin infiltration by donor $\mathrm{T}$ cells (Figure 62). As before, at D+7 post-transplant samples from skin and spleen were collected and analysed by flow cytometry.

As illustrated in Figure 63, Notch signalling blockade induced a decrease in IFN-y production by donor $\mathrm{CD}^{+} \mathrm{T}_{\text {eff }}$ cells in the skin, particularly in the epidermis (DMSO: $23.8 \% \pm 9.5$ vs LY411575: $11.2 \% \pm 2.5$; mean \pm SD), whereas it had no significant effect in the spleen or in the gut. These findings reinforce the idea that donor $\mathrm{T}$ cell pathogenicity is determined by distinct tissue specific / selective signals which regulate $\mathrm{T}$ cell effector function. 
Table 15. Top 10 type I and type II IFN responsive genes upregulated by LC after alloBMT.

\begin{tabular}{llc}
\hline Gene Symbol & Description & Log2 Fold Change \\
\hline \hline ligp1 & Interferon inducible GTPase 1 & 25.66 \\
Cxc19 & Chemokine (C-X-C motif) ligand 9 & 20.76 \\
Ifi202b & Interferon activated gene 202B & 13.70 \\
Ifi205 & Interferon activated gene 205 & 11.94 \\
Gbp2 & Guanylate binding protein 2 & 9.89 \\
Cish & Cytokine inducible SH2-containing protein & 8.36 \\
Cxcl10 & Chemokine (C-X-C motif) ligand 10 & 7.95 \\
Ccl22 & Chemokine (C-C motif) ligand 22 & 6.49 \\
Ccr7 & Chemokine (C-C motif) receptor 7 & 5.76 \\
Oasl2 & 2'-5' oligoadenylate synthetase-like 2 & 5.48 \\
\hline
\end{tabular}

Courtesy of Dr Thomas Conlan 


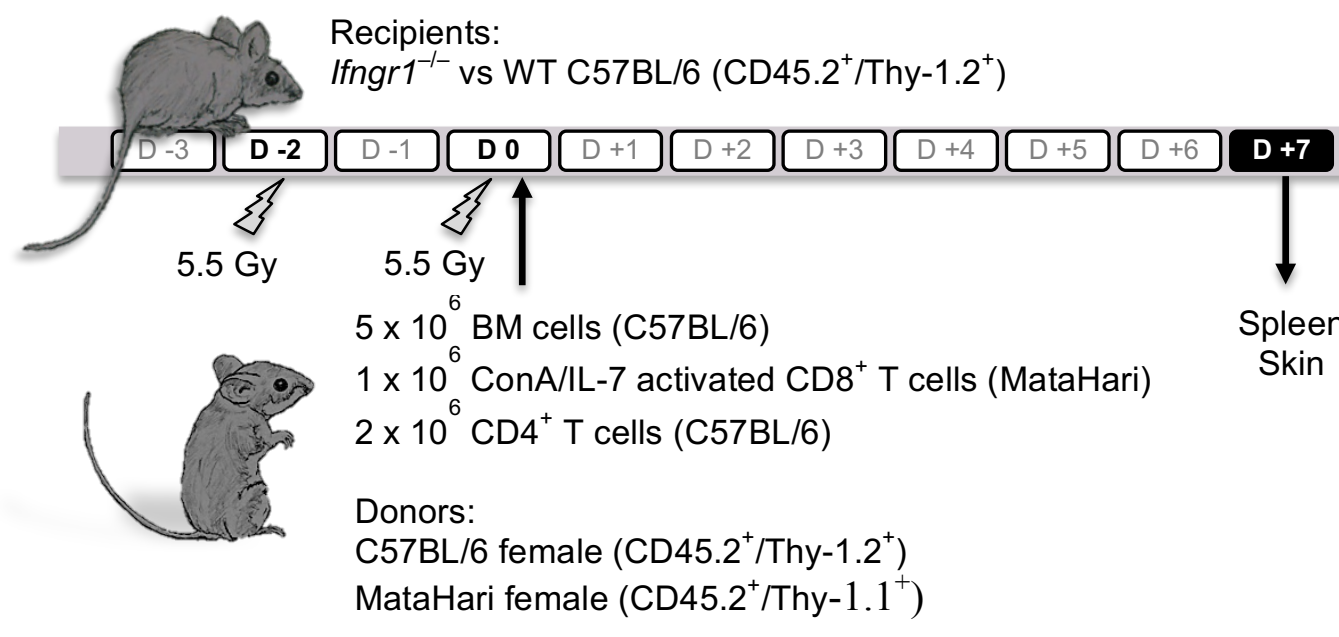

Figure 58. Experimental design to evaluate the requirement of LC responsiveness to IFN- $y$ for donor $\mathrm{CD}^{+} \mathrm{T}$ cell accumulation in the epidermis. 


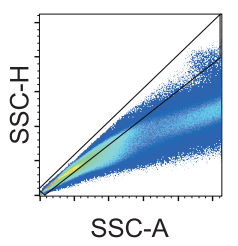

A

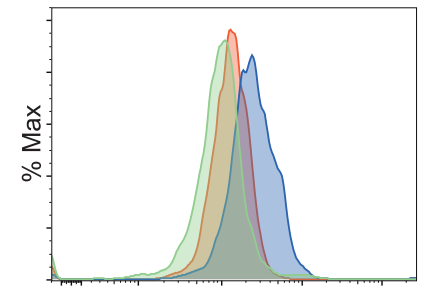

$\mathrm{H}-2 \mathrm{Db}$

B

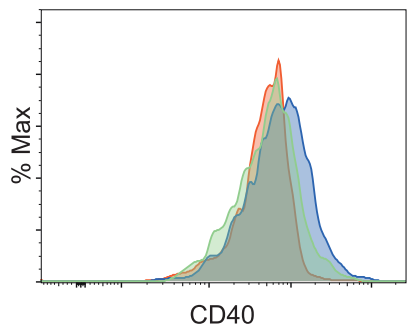

C

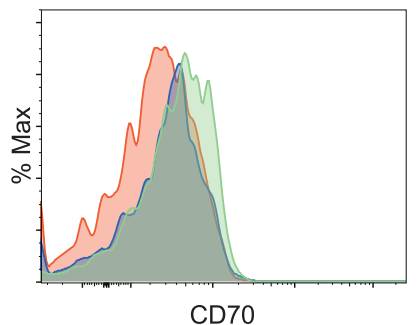

D

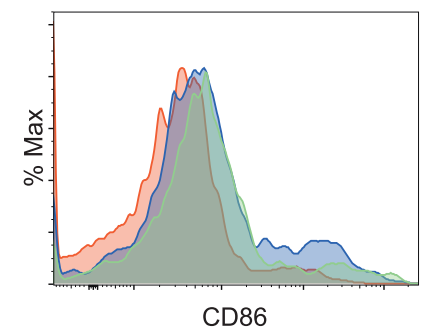

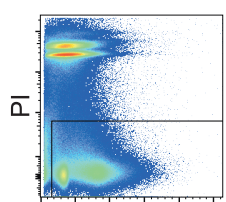

FSC-A

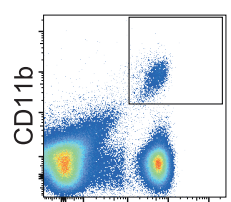

CD45

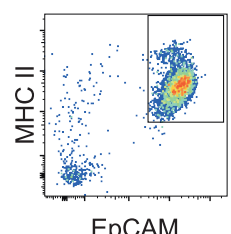

EpCAM
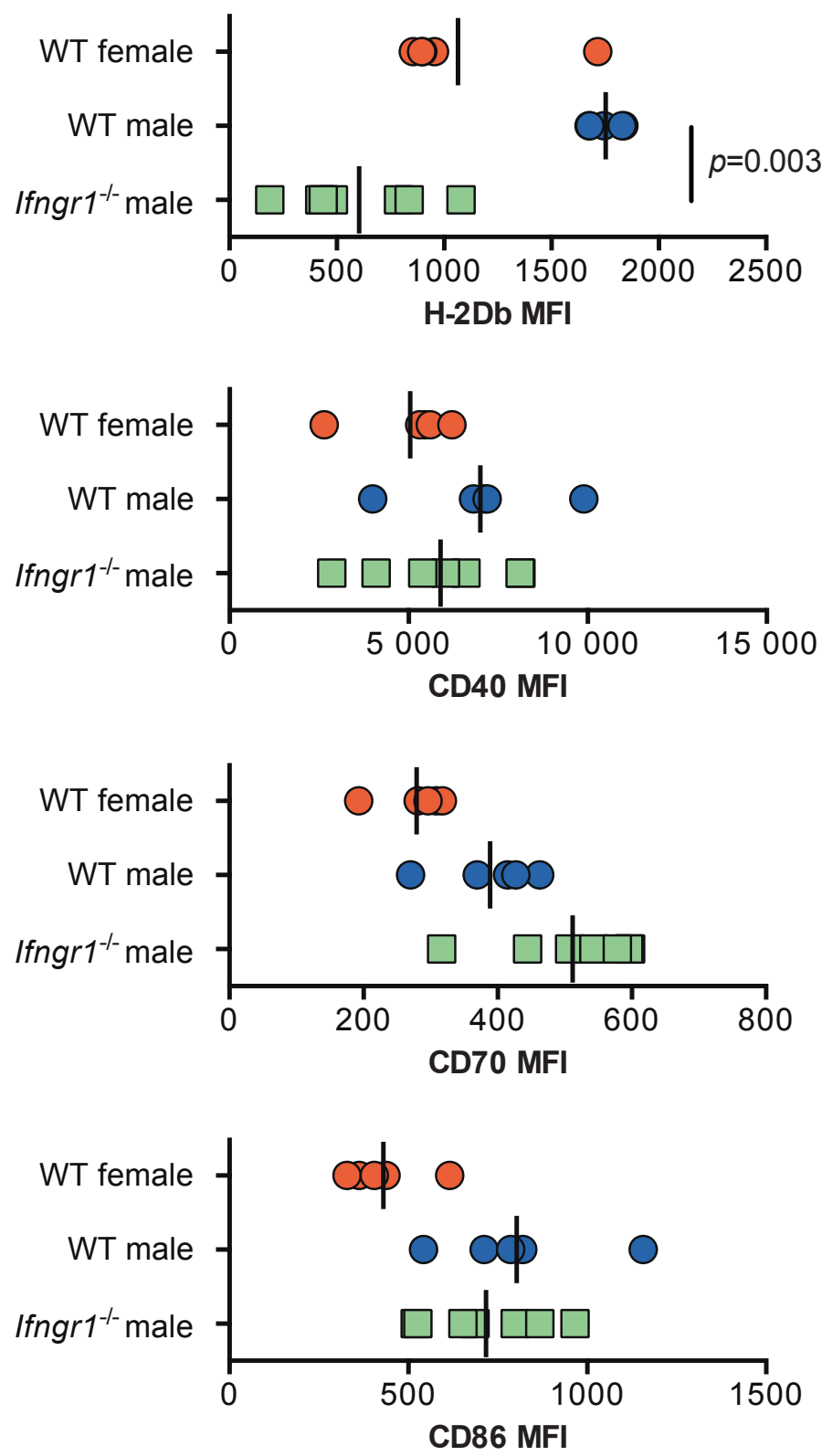

WT female

WT male

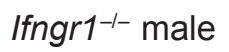

Figure 59. Comparison of alloBMT driven changes in LC expression of MHC class I H-2Db (A), CD40 (B), CD70 (C) and CD86 (D). Gating strategy used to identify LC, representative histograms of cell surface staining and summary data are shown. When unable to detect IFN-Y signalling (Ifngr $1^{-1-}$ ), LC fail to up-regulate MHC class I. WT female (synBMT control): $n=5$; WT male (alloBMT): $\mathrm{n}=5$; Ifngr $^{-{ }^{-l}}$ (alloBMT): $\mathrm{n}=7$; data pooled from 2 independent experiments. 


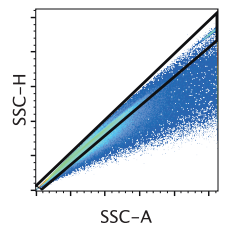

Spleen

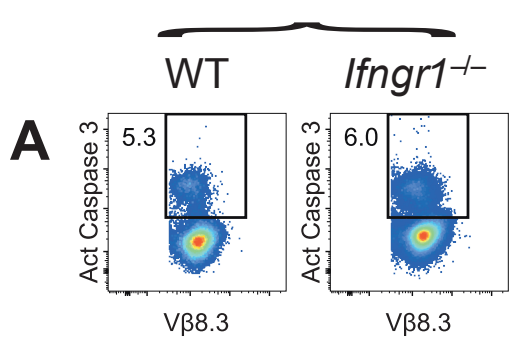

B

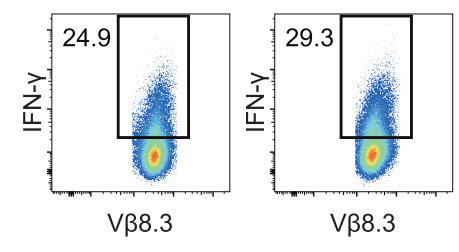

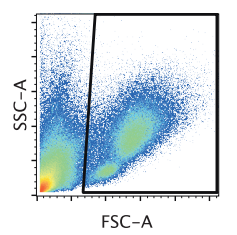

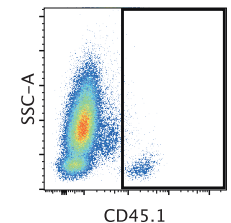

Dermis
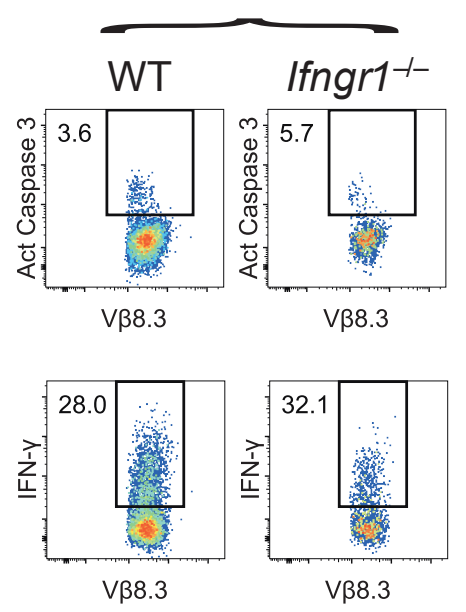

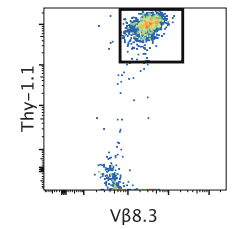

\section{Epidermis}
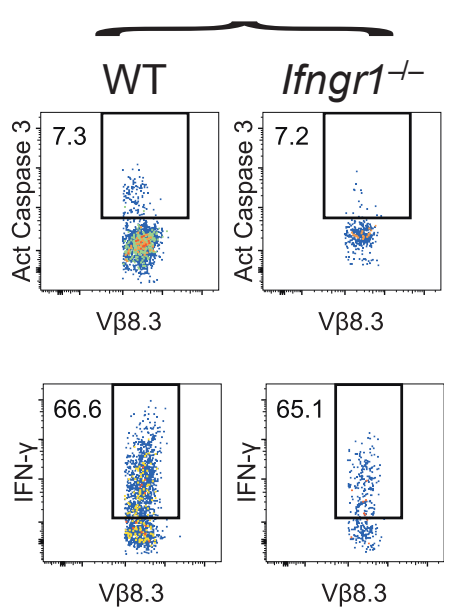

Figure 60. Study of the survival (A) and effector function (B) of donor $\mathrm{CD} 8^{+} \mathrm{T}$ cells isolated from alloBMT recipients incapable of detecting IFN- $y$ signalling $\left(\right.$ Ifngr $\left.1^{-1-}\right)$ vs WT. Samples from the spleen, dermis and epidermis were stained active caspase-3 and IFN- $y$. The gating strategy used to identify donor $\mathrm{CD}^{+} \mathrm{T}$ cells and representative plots for each group and condition are shown. 
A

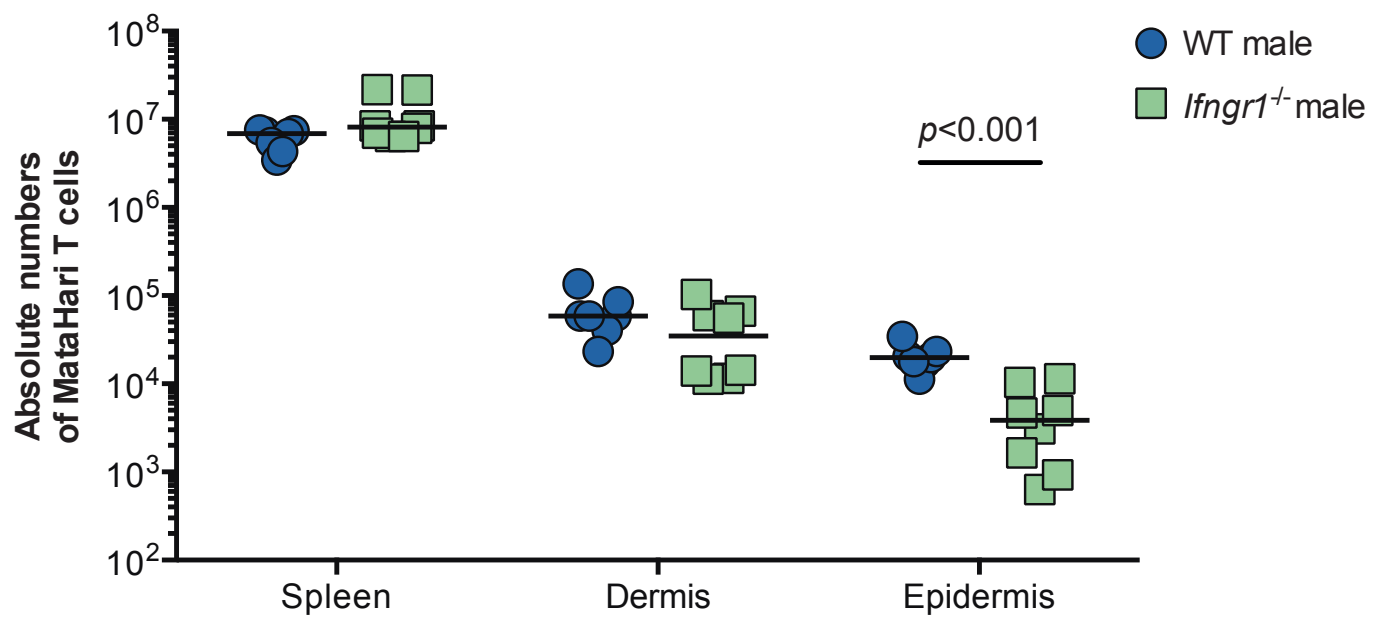

B

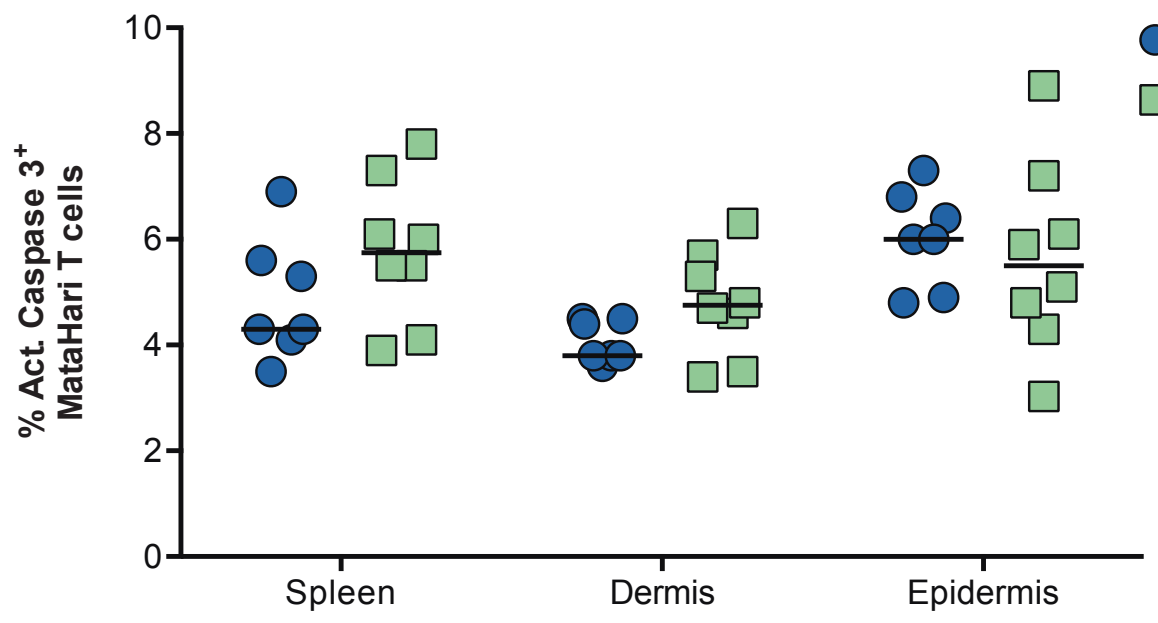

C

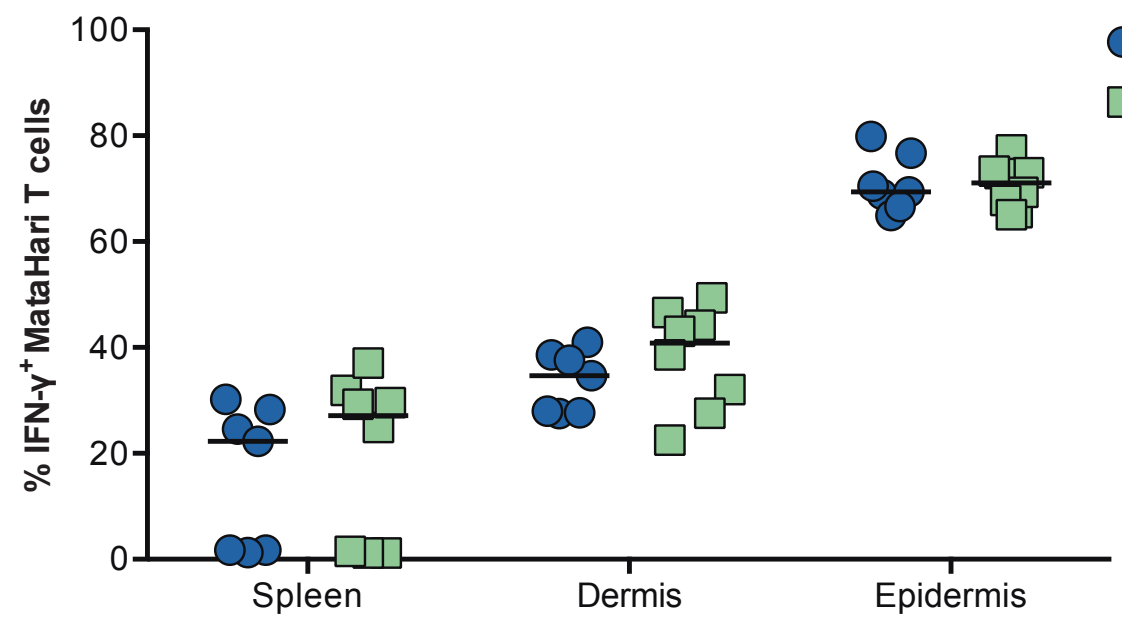

Figure 61. Summary data refering to the study of the effect of LC responsiveness to IFN-Y signalling upon accumulation (A), survival (B) and effector function (C) of donor $\mathrm{CD} 8^{+} \mathrm{T}_{\text {eff }}$ cells. Note the significant reduction in tissue infiltrating donor $\mathrm{T}$ cells specifically in the epidermis of Ifngr $^{-1-}$ recipients. No differences in donor $\mathrm{CD}^{+} \mathrm{T}$ cell survival or effector function was observed between the groups. WT male: $\mathrm{n}=7$; Ifngr $^{-1-}: \mathrm{n}=8$; data pooled from 2 independent experiments. 


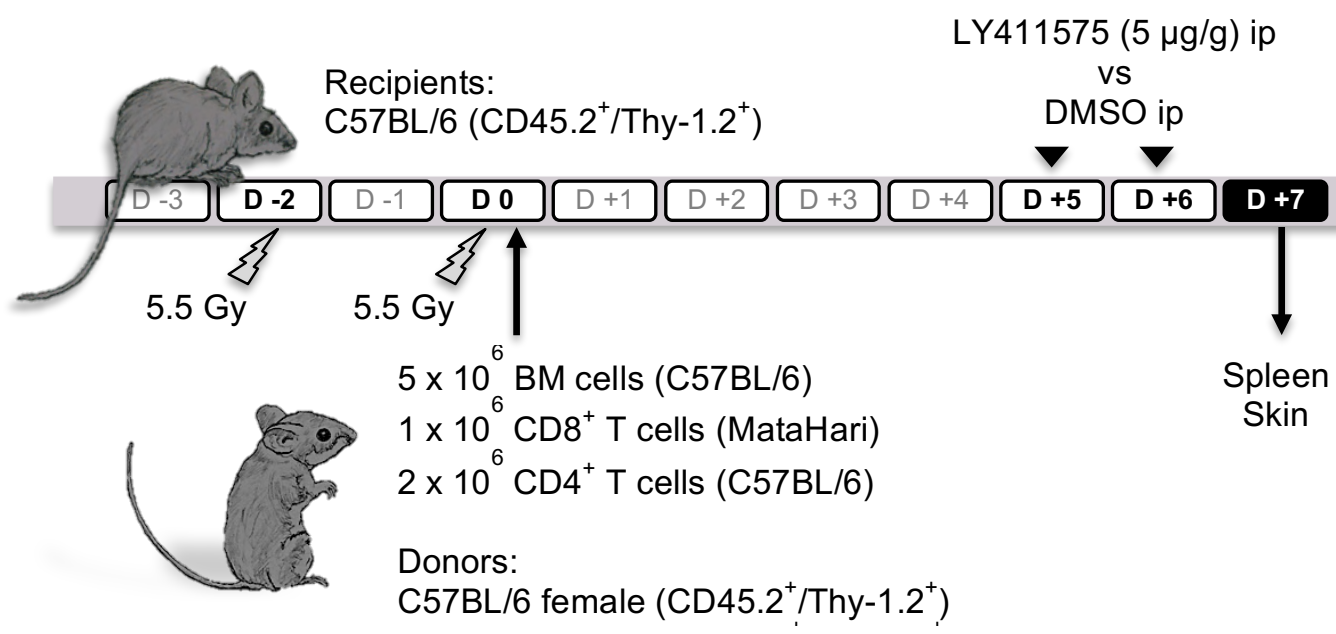

Figure 62. Experimental design to evaluate the effect of Notch signalling blockade on donor $\mathrm{CD} 8^{+} \mathrm{T}$ cell effector function in the epidermis. 
A
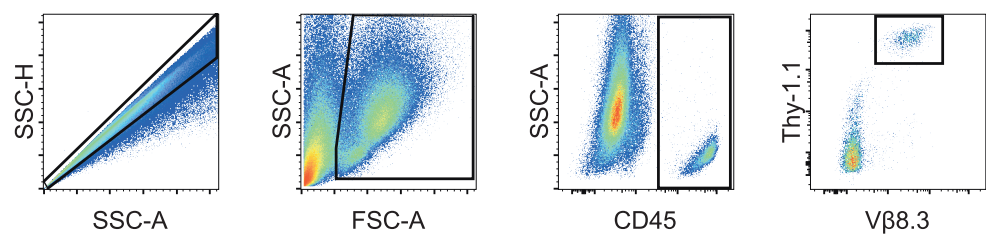

Spleen

\section{LP}

IEL

Dermis

Epidermis

DMSO
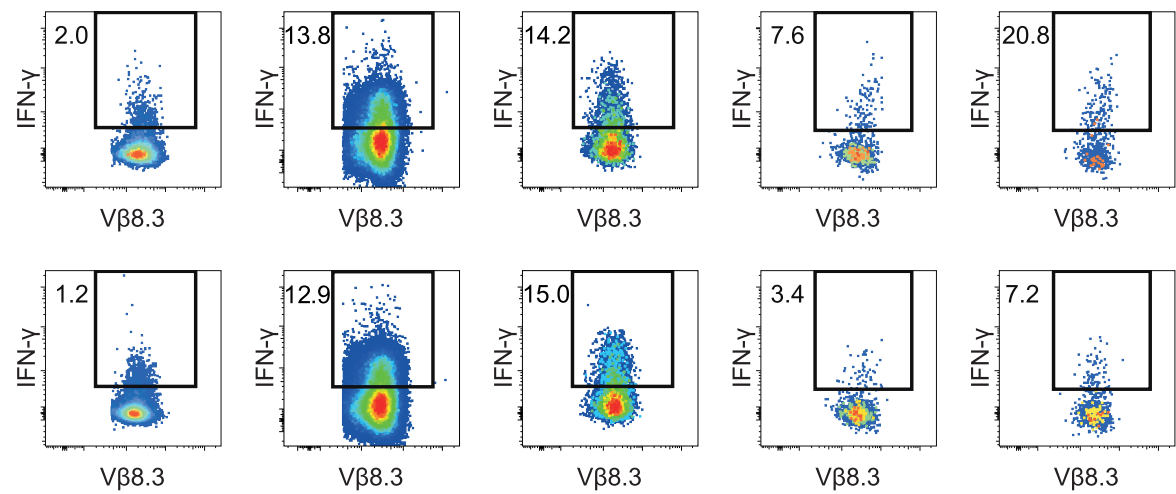

B

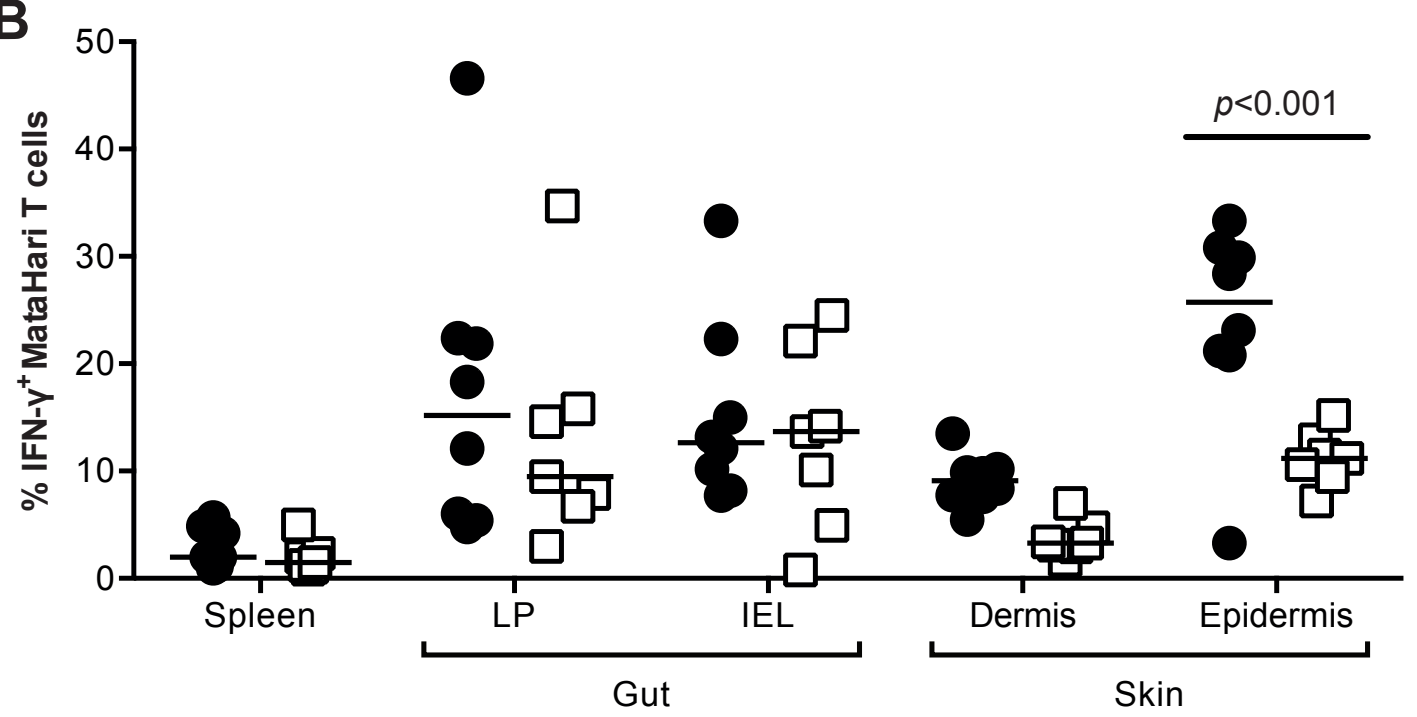

DMSO

LY411575

Figure 63. Study of the effect of Notch signalling blockade on donor $\mathrm{CD} 8^{+} \mathrm{T}$ cell effector function in the spleen, dermis and epidermis. (A) Gating strategy used to identify donor CD8 ${ }^{+}$ $T$ cells and representative plots for each tissue and condition. (B) Summary data. Treatment with LY411575 dramatically reduced donor $\mathrm{CD}^{+} \mathrm{T}$ cell IFN-y production in the epidermis, with no statistically significant effect on the other tissues. DMSO (control): $n=8$; LY411575: $n=7$; data pooled from 3 independent experiments. 


\subsection{Discussion}

Prior work in various infection models has documented the enhancement of the effector function of T cells when they enter the peripheral tissues. McGill et al. and Dolfi et al. have shown that after initial activation in the LN, influenzaspecific $\mathrm{CD}^{+} \mathrm{T}$ cells require additional antigen-dependent interactions with $\mathrm{DC}$ in the lungs for the establishment of an effective protective response. ${ }^{(222-224)}$ Likewise, in a model of LCMV meningitis, Kang et al. observed that cognate peptide-MHC I interactions occurring in the meninges influenced cytotoxic $\mathrm{T}$ cell division in situ; ${ }^{(322)}$ in the skin, McLachlan et al. showed that CD11 ${ }^{\text {hi }}$ dermal dendritic cells' antigen presentation in situ drives IFN- $\gamma$ production by cutaneous $\mathrm{T}_{\text {eff }}$ cells. ${ }^{(225)}$

It is conceivable that this "two-hit" model of the immune response is also applicable in the context of GVHD and it is supported by observation of a dichotomy between the transcriptional profiles of $\mathrm{T}_{\text {eff }}$ cells in SLO and GVHD target organs, which reflected an up-regulation of effector function genes and pathways in the peripheral tissues. Moreover, in the particular case of the skin, this study identified distinct transcriptional signatures for $T_{\text {eff }}$ cells isolated from the dermis or the epidermis.

Dr Thomas Conlan had demonstrated using the Langerin-DTR system that the severity of cutaneous acute GVHD was greatly reduced when host LC were depleted. ${ }^{(217)}$ The advantage of using the Langerin-DTR system is that by treating the recipients with DT at D-21, selective depletion of host LC is 
achieved as the repopulation kinetics of LC is considerably longer than other Langerin expressing cell populations. ${ }^{(323,324)}$ Following on his work, the same model was used to test the effects of LC depletion on the donor CD8 ${ }^{+} \mathrm{T}$ cells at the gene expression level.

DT treatment did not alter significantly $T_{\text {eff }}$ cell transcriptome in the $L N$ and dermis, yet it produced a profound shift in the gene expression of $\mathrm{T}_{\text {eff }}$ cells in the epidermis (Figure 48 and Figure 49). This suggests that the changes induced by LC depletion were not due to the absence of migratory LC compromising priming events, but rather a result of the disruption of the epidermal niche. Interestingly the main effect observed was the abatement of the differences between the skin's two compartments: in a LC replete skin, the dermis-to-epidermis transition of $\mathrm{T}_{\text {eff }}$ cells was characterised by the up-regulation of a set of genes, identified by WGCNA as module 28, which failed to occur when LC had been depleted (Figure 51).

Consistent with the previous reports that LC depletion was associated with abrogation of cutaneous GVHD, comparison of the cellular programs activated in the epidermis of PBS or DT treated recipients revealed that host LC increased donor $\mathrm{CD}^{+} \mathrm{T}_{\text {eff }}$ cell pathogenicity through cognate antigen recognition, co-stimulatory signalling and cytokine-cytokine receptor interactions, thus promoting their accumulation and survival, and enhancing their effector function.

These results were validated by FACS in experiments which confirmed that the reduction in cutaneous GVHD severity was not only due to having lower numbers of donor $T_{\text {eff }}$ cells in the skin, in the absence of $L C, T$ cells infiltrating 
the epidermis were clearly more pro-apoptotic, produced lower levels of effector molecules such as IFN-Y (Figure 55), and had a reduced potential of differentiation into $\mathrm{T}_{\mathrm{RM}}$ cells (Figure 57).

Two main features were identified as being central to this $L C-T_{\text {eff }}$ cell crosstalk: LC responsiveness to IFN-y, required for DC maturation ${ }^{(325)}$ and up-regulation of $\mathrm{MHC}$ class $\mathrm{I}^{(326)}$ and signalling through the Notch pathway, recognised as a critical regulator of allogeneic $T$ cell responses in GVHD. ${ }^{(319,321,327)}$

Indeed, in the absence of IFN-Y receptor signalling, LC failed to up-regulate $\mathrm{H}-2 \mathrm{Db}$ expression and $\mathrm{T}_{\text {eff }}$ cell accumulation in the epidermis was reduced (Figure 60 and Figure 61). It could be argued that the defect in $\mathrm{T}_{\text {eff }}$ cell accumulation observed in the epidermis was a result of impaired expansion of donor $\mathrm{T}$ cells in a system unresponsive to IFN-y signalling. However, no differences in the absolute numbers of donor T cells were detected in the spleen and dermis, suggesting that in vitro ConA/IL-7 activation of donor T cells was successful in overcoming any priming deficits inherent to the Ifngr $1^{-/-}$system.

It has been previously reported that Notch signalling blockade reduces the severity of GVHD by inducing a hyporesponsive program in $T$ cells. ${ }^{(318,319,321)}$ However, these studies were performed either by using genetic models or prolonged pharmacological and antibody-mediated treatments which produce a sustained suppression of Notch signalling affecting not only $T_{\text {eff }}$ cells function but all developmental stages. The alternative approach adopted here (treatment with a GSI only from D+5 onwards) was aimed at preserving the priming events which usually occur in the first 3 days after T cell transfer and 
allow for a normal infiltration and early accumulation of donor $\mathrm{T}$ cells in the peripheral tissues. Moreover, in these studies only the lymphoid organs (spleen and lymph nodes) were evaluated and the differences in IFN-y production do not accurately reflect a decrease in $\mathrm{T}$ cell effector function in vivo as all measurements were performed after re-stimulation either with anti-CD3/antiCD28 or phorbol 12-myristate 13- acetate and ionomycin. By performing direct ex vivo measurements (without re-stimulation) of intracellular IFN-y in donor $\mathrm{CD}^{+} \mathrm{T}_{\text {eff }}$ cells from the various sites, it was possible to evaluate the true effect of Notch signalling blockade in each compartment. With this protocol (Figure 63), it became evident that the production of IFN-y donor $\mathrm{CD}^{+} \mathrm{T}_{\text {eff }}$ cells is only detectable in the GVHD target organs. Interestingly, the GSI treatment did not impact all the compartments evenly: while there was a consistent reduction in the levels of IFN-Y in the skin, particularly in the epidermis, in the gut the production of IFN-y remained mostly unaffected. These results are consistent with the proposed modular architecture of donor $\mathrm{T}_{\text {eff }}$ cells gene expression during GVHD development, in which independent programs would be autonomously regulated. The epidermis specificity of the GSI treatment effect upon IFN-y production, mirroring the changes in effector function observed in donor $\mathrm{CD}^{+} \mathrm{T}$ cells induced by LC depletion, furthers the hypothesis that, in the skin, Notch signalling is a potential dominant pathway involved in the host LC - donor T cell cross talk.

Altogether, the data demonstrate that, in the skin, GVHD is defined by tissue-autonomous instructions dictated by $\mathrm{T}_{\text {eff }}$ cell migration to the epidermis and interaction with LC in situ, and is regulated by Notch-dependent signals to enhance local cytokine production by $\mathrm{T}_{\text {eff }}$ cells and Notch-independent signals 
to promote $\mathrm{T}$ cell accumulation and resistance to apoptosis. Although the results strongly suggest that LC are the central participants in this cross-talk with donor $\mathrm{CD}^{+} \mathrm{T}$ cells, to fully characterise this mechanism, further work is required in models allowing for inducible and cell selective inhibition of these signalling pathways. 
IV. Discussion 


\subsection{Systems immunology - a comprehensive approach to acute GVHD study}

The aim of this project was to undertake an unbiased and systems-wide methodology to defining the cellular mechanisms underlying tissue-specific pathology in GVHD. The primary hypothesis being tested was that GVHD target tissues exert dominant, idiosyncratic roles in regulating effector T cell functions.

The approach taken, involving gene expression profiling of donor $\mathrm{CD} 8^{+}$ $\mathrm{T}$ cells isolated at key time points of the alloreactive response development (before $\mathrm{T}$ cell transfer - naïve donor $\mathrm{T}$ cells; $\mathrm{D}+3$ - early post-priming; $D+6 / D+7$ - peak of peripheral tissue $T$ cell infiltration) and from a variety of tissues (secondary lymphoid organs, blood, and GVHD target organs), permitted the characterisation of the transcriptional response of donor T cells during the evolution of GVHD.

For this purpose, two well characterised murine models of GVHD were used which reproduce the clinical features and histological abnormalities found in patients with acute GVHD. The models were selected for their clinical relevance, as the $\mathrm{B} 6 \rightarrow 129$ model, ${ }^{(267,268)}$ being a multiple minor histocompatibility antigen mismatched BMT model, is similar to a standard HLA-identical sibling SCT, ${ }^{(328)}$ and the MataHari T cell model, ${ }^{(118)}$ being a single 
minor histocompatibility antigen mismatched BMT model, simulates female $\rightarrow$ male HLA-identical SCT which is associated with higher risk of GVHD development. ${ }^{(329,330)}$

Exploratory analysis of the data revealed a remarkable heterogeneity in the gene expression of donor $\mathrm{CD}^{+} \mathrm{T}_{\text {eff }}$ cells. At $\mathrm{D}+7$ post-transplant, a clear dichotomy between the transcriptional profiles of SLO and GVHD target organs was identified, underlining a distinct $\mathrm{T}_{\text {eff }}$ cell differentiation pattern according to their location (Figure 22 and Figure 24). These differences were found to be independent of the TCR repertoire and of antigen distribution, and reflected an enrichment for effector function related genes in the peripheral tissues (Figure 26 and Figure 28).

Such findings challenge the SLO-centric classical scheme of acute GVHD development, ${ }^{(331,332)}$ which envisages the alloreactive response as a sequence of events: (1) priming of the immune response, entailing APC activation and maturation; (2) donor $\mathrm{T}$ cell activation, proliferation and differentiation; (3) migration of $\mathrm{T}_{\text {eff }}$ cells and other effector leukocytes to GVHD target tissues; and (4) target tissue destruction by $\mathrm{T}_{\text {eff }}$ cells. ${ }^{(81,90,91)}$ According to this model, the full process of effector differentiation would be determined by priming, so T cells entering the peripheral tissues would already be hard-wired to cause tissue damage. However, contrary to what would have been anticipated, a great diversity in $T_{\text {eff }}$ cell transcriptional profiles was observed among the tissues which could not be explained by an early egress of effector cells from the SLO to the GVHD target organs (Figure 32), and were not indicative of homing imprinting idiosyncrasies (Figure 33). 
Thus far, transcriptome data analysis in GVHD has been focused on the blood and SLO, and has been based on gene expression level comparison between groups followed by description of the gene ontology of the transcripts found to be significantly differentially expressed. ${ }^{(298,333-335)}$ However, this methodology underestimates the differences in the system, disregarding any change that does not meet the preconceived thresholds. Moreover, by failing to recognise patterns of correlated gene expression, it lacks insight into important biological aspects of the disease pathogenesis.

WGCNA is a powerful analytical tool which groups genes into modules on the basis of their coexpression similarities. ${ }^{(228)}$ This systems biology methodology has been proven effective in identifying functionally related genes, hence enabling a more systematic and global interpretation of gene expression data. ${ }^{(230,231)}$

In the present study, 31 modules of genes were identified by WGCNA and, remarkably, their value in representing gene expression changes occurring during alloreactive response development in independent models of GVHD was largely validated. Not only were the majority of the modules shown to be preserved in a second murine model of GVHD (Figure 39), but, more importantly, the ones with the strongest preservation statistics accurately segregated synBMT from alloBMT samples (Figure 40), and identified those with GVHD (Figure 41), in published data sets from Rhesus macaques and human samples. ${ }^{(298)}$ Of note, a statistically significant association was found between several SLO-specific modules and blood samples from GVHD 
affected subjects. In contrast, no GVHD target organ-specific modules were enriched in the blood from either rhesus macaques or humans.

Due to the paucity of published transcriptional data on $\mathrm{T}_{\text {eff }}$ cells infiltrating the peripheral tissues, validation of the GVHD target organ-specific modules required a collaboration with Dr Laura Jardine and Dr Mathew Collin from the Newcastle University Institute of Cellular Medicine who provided paired samples of $\mathrm{CD}^{+} \mathrm{T}$ cells isolated from blood and skin biopsies of patients with GVHD. Likewise, SLO-specific modules were found to be enriched mainly in the blood samples, while the GVHD target organ-specific modules were over-represented in the epidermis (Figure 43). 


\subsection{A modular conception of the cellular programs underpinning GVHD development}

Analysis of SLO- and GVHD target organ-specific modules has broadened the understanding of GVHD biology, providing valuable insight into cellular programs reported to be associated with GVHD in previous studies, and aiding to the mapping of the alloreactive response.

For instance, module 1, associated with the blood and bone marrow, was related to the regulation of the cell cycle, and included Cdk1, Aurka and Aurkb as driver genes, lending support to the concept proposed by Li et al. that cyclin-dependent kinases are required for alloreactive antigen-specific $\mathrm{T}$ cell clonal expansion, ${ }^{(336)}$ and to Furlan et al. observation that aurora kinase control of $T$ cell turnover is dysregulated during GVHD. ${ }^{(298)}$ Furthermore, the LN associated module 17 was linked to Toll like receptor and retinoic acid-inducible gene 1 signalling, two extensively studied type I IFN inducing pathways recognised in the literature as playing an important role in the pathogenesis of GVHD. ${ }^{(104,337)}$ Interestingly, modules 3 and 4 , which are mostly related to oxidative phosphorylation and fatty acid metabolism, were positively correlated with $T_{\text {eff }}$ cells from the spleen, bone marrow and lymph nodes, but negatively correlated with $\mathrm{T}_{\text {eff }}$ cells from the gut and skin, suggesting that the dependence 
of alloreactive T cells upon fatty acid oxidation described by Byersdorfer et al. may be limited to the SLO. ${ }^{(338)}$

In contrast to the priming and proliferation programs that characterised the SLO, GVHD target organ-associated modules were mostly involved in pro-inflammatory cytokine generation. Notably, the pan-GVHD target organ associated module 29 included multiple transcription factor genes that regulate Th17/Tc17 fate, e.g. Fos/2, Kdm6b, Skil and Chd7. ${ }^{(339-341)}$ Additional Th17/Tc17-related transcription factors, such as $/ k z f 3$, were identified as driver genes of module 31, reinforcing the role of Tc17 differentiation program during GVHD, particularly in the gastrointestinal tract. ${ }^{(287)}$ On the other hand, in the skin, the epidermis-specific module 28 driver genes were predominantly linked to T cell activation (I/2ra, Tnfs9, Cd44), Th1-type pro-inflammatory cytokine generation (Ifng, Csf1 and Csf2), glycolysis (Pkm2), Notch signalling (Rpbj, Furin), and survival (Bc/2/1).

The diversity of transcription profiles and the organ specificity of cellular programs in GVHD uncovered by this novel approach suggests that $\mathrm{T}$ cell function is driven by compartment-specific interactions occurring at each site. However, further work is required to understand the regulatory mechanisms responsible for driving this spatial diversity of effector $\mathrm{T}$ cell programs. One attractive research avenue would be to integrate the gene expression data obtained in this study with multi-omic data from public repositories to define gene regulatory networks and develop working models capable of explaining and predicting gene expression, and simulating the effect of specific changes in silico. ${ }^{(342)}$ For instance, the data from published ChIP-seq studies now 
available as libraries ${ }^{(343,344)}$ containing information on transcription factors, their genomic binding sites and DNA binding profiles, could be used to probe the gene modules and identify the transcriptional networks that regulate each program. Additionally, it would be of great value to analyse the DNA and histone modifications associated with each compartment and study their relationship with the patterns of program activation/silencing observed, considering the emergent information concerning the characterisation of the epigenomic landscapes in primary cells and tissues. ${ }^{(345)}$ 


\subsection{A "two-hit" model of cutaneous acute GVHD development}

To investigate the mechanisms of induction of the tissue-specific programs, the study was focused on the skin as it displayed one of the most striking differences between compartments, and its associated modules, in particular module 28, were also enriched in samples collected from human GVHD patients.

The key histopathological findings that characterise acute cutaneous GVHD include a sparse to extensive lymphocytic infiltrate which commonly involves the epidermis and adnexal structures, accompanied by hydropic changes to the basement membrane and keratinocyte necrosis. ${ }^{(346)}$ Similar changes were observed in the MataHari T cell model of GVHD, ${ }^{(118)}$ with donor $\mathrm{CD}^{+} \mathrm{T}_{\text {eff }}$ cells spanning the dermis-epidermis interface, in close proximity to the radio-resistant host-derived LC (unpublished data).

It has long been recognised that host APC are required for the induction of acute GVHD, ${ }^{(112)}$ while donor derived APC are mostly involved in perpetuating tissue injury. ${ }^{(113)}$ However, in the particular case of the skin, this subject is still controversial: while Merad et al. showed that host LC were sufficient to cause skin GVHD, ${ }^{(214)} \mathrm{Li}$ et al. demonstrated that LC were in fact 
not required to induce GVHD, as other subsets of APC were equally able to prime T cells. ${ }^{(215)}$ However, in both studies the observations reported were based on experimental models in which the normal skin immune niche had been extensively remodelled. In the former, by using MHC-mismatched donor $\rightarrow$ host BM chimeras as recipients for a second GVHD-inducing transplant, Merad et al. created a state in which the only residual recipient APC at the time of the second transplant were the LC. On the other hand, Li et al. used a transgenic model in which LC are constitutively depleted (Langerin-DTA system), disregarding any changes that this determined to the skin immune baseline.

In contrast, the Langerin-DTR model developed by Dr Clare Bennett allows for the selective depletion of LC when recipients are treated with DT at least 21 days prior to transplantation. ${ }^{(216)}$ By allowing 3 weeks of rest, all other populations of Langerin ${ }^{+}$cells, including the Langerin $^{+}$dermal DC, are regenerated and have reached steady state equilibrium at the time of BMT. ${ }^{(323,324)}$ Using these transgenic mice as recipients of minor histocompatibility antigen mismatch BMT (MataHari T cell model), it was demonstrated that host LC were indeed required for donor $\mathrm{CD}^{+} \mathrm{T}_{\text {eff }}$ cell accumulation in the epidermis, while host Langerin ${ }^{+}$dermal dendritic cells were found to be redundant. ${ }^{(217)}$ Moreover, it was observed that the severity of cutaneous GVHD was greatly reduced when LC were absent, even when only localised LC depletion was performed. ${ }^{(217)}$

These results suggest that in situ interactions between donor $\mathrm{CD}^{+} \mathrm{T}_{\text {eff }}$ cells and host LC were pivotal for the regulation of tissue damage in skin GVHD. 
This concept that $\mathrm{T}$ cell effector function is enhanced in the peripheral tissues through antigen-dependent interactions with resident DC conforms to the "two-hit" model of the immune response which has already been well-documented in several studies on inflammation and infection. ${ }^{(222-225,322)}$ It was thus hypothesised that the differences detected in the transcriptional profiles of $\mathrm{T}_{\text {eff }}$ cells isolated from the two skin compartments, dermis and epidermis, were dictated by LC.

Indeed, with LC depletion the contrast between the gene expression profile of dermal and epidermal donor $\mathrm{CD}^{+} \mathrm{T}_{\text {eff }}$ cells was attenuated. It was found that migration of $T_{\text {eff }}$ cells from the dermis to the epidermis enhanced their pathogenicity and was linked to the expression of a broad effector program, characterized by cell cycle commitment, mRNA synthesis and increased metabolic activity (Figure 50). However, in the absence of LC, donor CD8 ${ }^{+} \mathrm{T}$ cells infiltrating the epidermis were rendered incapable of up-regulating the full array of effector genes (Figure 51), had a reduced survival (Figure 54), and showed impaired differentiation into resident memory cells (Figure 57).

Consistent with the hypothesis of LC- $T_{\text {eff }}$ cell crosstalk, a complementarity of signalling pathways was predicted from the gene expression profiles of the two cell types: Ifng was a driver gene of module 28 in $\mathrm{T}_{\text {eff }}$ cells; and multiple IFN-y responsive genes were up-regulated in host LC in the context of alloBMT. Despite the caveats of the model used (Ifngr ${ }^{-/-}$mice), pilot experiments showed that in the absence of IFN-y receptor signalling, LC failed to up-regulate $\mathrm{H}-2 \mathrm{Db}$ expression and $\mathrm{T}_{\text {eff }}$ cell accumulation in the epidermis was significantly reduced, even though donor T cell expansion in the 
SLO and infiltration of other peripheral tissues was not affected (Figure 59 and Figure 61).

Furthermore, in line with the results from various groups which reported Notch signalling as being central in the development of alloreactive T cells, ${ }^{(318-}$ 320) particularly for IFN-Y production, ${ }^{(321)}$ two key regulators of the Notch pathway (Rbpj and Furin) were identified as possible drivers of module 28 expression in the epidermis. These observations were validated by Notch signalling blockade experiments through treatment with a GSI, which induced a significant reduction of IFN-y production in the skin (Figure 63). By delaying GSI treatment until D+5, preservation of priming events and migration of T cells to the peripheral tissues was achieved, as no differences in donor $\mathrm{CD}^{+} \mathrm{T}_{\text {eff }}$ cell accumulation was observed in any of the studied tissues.

It is recognised that the models used to test the requirement of host IFN- $\mathrm{y}$ responsiveness and Notch signalling are non-specific, and that further work is needed to ascertain their relevance in LC regulation of donor $T_{\text {eff }}$ cell pathogenicity. However, given that the results consistently mirror the changes observed when LC are depleted, it is proposed that these are instrumental pathways central to the $L C-T_{\text {eff }}$ cell crosstalk. 


\subsection{Study limitations \& Future work}

Although the paramount role of experimental models in the progress of our understanding of immune system complexity is undeniable, and in providing important insight into the pathophysiology of immune mediated diseases, caution must be taken when interpreting the results obtained in animal studies. In the particular case of GVHD, to draw conclusions from murine models and translate them into the scenario of clinical GVHD, it is necessary to consider 5 potential caveats: $(90,191)$

a) Differences in conditioning regimens used to prepare the recipient.

While in humans pre-transplant conditioning regimens consist mainly of chemotherapy, with or without low rate-delivered fractioned TBI, in mouse models of GVHD irradiation alone is typically used with large fraction doses and high dose rates. This disparity in conditioning regimens results in important differences in the extent of the tissue damage and proinflammatory reaction, which may influence the GVHD phenotype. In all BMT experiments performed in this project, a split dose of irradiation was delivered to the recipients with a $48 \mathrm{~h}$ interval between fractions. Moreover, characterisation of the GVHD phenotype was performed in both models to ascertain that it mirrored the clinical and histological features observed in human patients. 
b) Donor-recipient genetic and immunologic disparities.

In humans, the majority of HSTC performed are matched by high-resolution HLA typing at the allele level for several MHC loci, but mismatched for miHAs. To reproduce these conditions, various $\mathrm{MHC}$ and/or miHA-disparate models have been created through the combination of different inbred mouse strains. It has been shown that different combinations of strains result in a variety of GVHD phenotypes which depend greatly on the MHC type and immunodominant target antigens. Such differences are known to determine the TCR repertoire and polarisation of donor T cells. ${ }^{(351)}$ Awareness of these strain-specific variations is critical for the comparison of results between models. Moreover, mice and humans differ in many aspects of the immune system, not only in terms of cell populations present at each niche (e.g. the mouse skin is primarily populated by DETC, whereas in humans $\alpha / \beta$ $\mathrm{T}$ cells predominate), but also in the expression of co-stimulatory molecules and chemokine receptors (e.g. CD28 is expressed by nearly all mouse $\mathrm{CD}^{+}$and $\mathrm{CD}^{+} \mathrm{T}$ cells, while in humans it is restricted to only $80 \%$ of $\mathrm{CD}^{+}$and $50 \%$ of $\mathrm{CD}^{+} \mathrm{T}$ cells). Additionally, the non-haematopoietic compartment also displays important species-specificities (e.g. in contrast to mice, human endothelial cells express both $\mathrm{MHC}-\mathrm{I}$ and $\mathrm{MHC}-\mathrm{II}$ molecules constitutively, and are thus able to present antigen both to $\mathrm{CD}^{+}$and $\mathrm{CD}^{+} \mathrm{T}$ cells), which make some observations made in animal models difficult to translate into the human system. ${ }^{(352)}$ 


\section{c) Source of donor immune cells.}

The graft for HSCT in humans is most commonly derived from mobilized stem cell products containing circulating donor immune cells. On the other hand, typically, in murine models spleen cells and/or lymph node cells are delivered together with the BM graft to ensure a sufficient dose of T cells for the induction of GVHD. Not only immune-cell populations from different sources may have different trafficking capacities, but, most importantly, differences in cell composition can have a great influence on GVHD phenotype. To mitigate these differences, in the BMT protocol used, the $\mathrm{BM}$ graft was solely enriched with purified $\mathrm{CD} 4^{+} \mathrm{T}$ cells and $\mathrm{CD} 8^{+} \mathrm{T}$ cells, in a 2:1 ratio.

\section{d) Differences in the microbial baseline.}

The kinetics and severity of GVHD, in particular intestinal GVHD, has been reported to be affected by the enteric pathogens. ${ }^{(347)}$ Notably, both the composition and the diversity of the enteric microbiome of humans and rodents are very different, as animals used in experimental models are, unlike humans, housed under specific-pathogen free conditions since birth. Moreover, transplanted patients are frequently required to undergo antibiotic prophylaxis and/or treatment which can alter significantly the enteric microbiome.

\section{e) Age of the donors and recipients.}

While the majority of HSCT are performed on adult humans, murine HSCT studies tendentiously use young adult mice, as older age in mice is associated with increased sensitivity to radiation, and reduced efficiency 
of immune reconstitution after transplant. For this project, all transplant recipients were 10 - 20 weeks old, and those used as donors were 8 - 16 weeks old.

Regarding the systems immunology approach, although WGCNA is a powerful tool to analyse microarray data sets, it requires an adequate pre-processing and normalization of the expression data and it can be biased when dealing with technical artifacts, tissue contamination or poor experimental design. ${ }^{(348)}$ In the case of this project, it was necessary to exclude tissue specific genes from the analysis. Despite the efforts to avoid preconceptions, it is recognised that by relying on gene ontology annotations or pathway information to analyse the modules, the characterisation of the cellular programs is incomplete and may show a bias towards what is already known. ${ }^{(349)}$

Although the SLO-associated modules were readily validated in a non-human model of GVHD and with human samples from transplanted patients with GVHD, testing of the value of GVHD target organ-associated modules was hindered by the lack of studies focusing on the peripheral tissues. The analysis performed in collaboration with the Newcastle University Institute of Cellular Medicine permitted confirmation that these modules were effective at distinguishing between $\mathrm{CD}^{+} \mathrm{T}$ cells isolated from the blood and skin of GVHD patients, but lacked the power to determine their correlation with individual compartments. Future work should therefore include follow-up studies designed with larger sample sizes comprising specimens from other GVHD target organs. 
In terms of the characterisation of the tissue-specific cues that control donor T cell effector function, this work provides compelling evidence that in the skin LC play a central role in regulating the development of cutaneous GVHD. Although the experiments performed in the Ifngr $^{-1-}$ model and with $\mathrm{GSI}$ treatment are insufficient to demonstrate the requirement of IFN- $\gamma$ and Notch signalling in the $\mathrm{LC}-\mathrm{T}_{\text {eff }}$ cell crosstalk, this work provides the framework for future studies in which these signalling pathways may be inhibited in inducible and cell selective systems.

Moreover, a better understanding of LC biology and their interactions with the remaining elements of the skin immune network will grant important insight into the mechanisms regulating the homeostasis of the skin's immune niche. A prominent subject of interest that would merit exploring is their effect on the skin's microbiota, as there is increasing data supporting a correlation between the risk of GVHD and the patient's microbiome diversity. ${ }^{(100,188,350)}$

Lastly, the classical prophylactic approaches to acute GVHD, such as treatment with methotrexate and cyclosporine/tacrolimus, has proved to be of great value in the prevention of GVHD. However, prolonged non-specific immunosuppression can impair the GVL response and hamper the reconstitution of the immune system, increasing the risk of relapse of the underlying neoplastic disease and of infectious complications. By demonstrating that GVHD is defined by tissue-autonomous regulation of effector $\mathrm{T}$ cells, the data presented here establishes the rationale for future studies aimed at identifying tissue-specific molecular targets which may translate into the prevention of the development of severe GVHD in the clinic. 
V. Conclusion 


\section{Conclusion}

Current knowledge of GVHD biology is based on research mainly focused on the events occurring in the SLO, using classical analytical methods that employ preconceived thresholds. Their capacity to identify novel and biologically meaningful pathways and cellular programs involved in GVHD pathogenesis has thus been limited, and the translational clinical benefit has been reduced. The systems immunology methodology explored in this thesis represents an innovative approach to the study of GVHD that not only takes into account the variability in donor $\mathrm{T}$ cell expression programs determined by their location (SLO versus GVHD target organs), but also characterises the cellular programs expressed at each site based on the analysis of a weighed correlation network of genes.

It was demonstrated that (1) the transcriptional profiles of donor CD8 ${ }^{+}$ $\mathrm{T}_{\text {eff }}$ cells in peripheral tissues (skin, gut, liver) were very distinct from those in the lymphoid organs (lymph node, spleen, bone marrow and blood), (2) these profiles diverged sharply between the different GVHD target organs, and (3) also differed between individual sub-compartments of single organs. This phenomenon was independent of the TCR repertoire, and did not reflect variations in antigen distribution, suggesting that $\mathrm{T}$ cell effector programs are driven by compartment-specific interactions occurring at each site. 
In the particular case of the skin, $L C-\mathrm{T}_{\text {eff }}$ cell crosstalk was shown to be central to the development of cutaneous acute GVHD, where responsiveness to IFN- $\gamma$ was required for LC maturation, while Notch-dependent signals enhanced local cytokine production by $T_{\text {eff }}$ cells and Notch-independent signals to promoted T cell accumulation and resistance to apoptosis.

Collectively, these data demonstrate that GVHD is defined by tissue-autonomous regulation of effector T cells and provides a rationale for the development of precision therapies directed at blocking GVHD in individual tissues, so that by avoiding global immune suppression, these approaches may permit preservation of GVT effects. 


\section{Acknowledgements}




\section{Acknowledgements}

First and foremost, I want to express my sincere gratitude to my supervisors Professor Ronjon Chakraverty, Dr Clare Bennett and Professor Stephen Mackinnon for accepting me as their PhD student and giving me the opportunity to work in their laboratory; and to Bloodwise and Fundação para a Ciência e Tecnologia (FCT Portugal), for their financial support, without which this work would not have been possible. I am particularly thankful to Professor Chakraverty and Dr Bennett for the frequent thought-provoking discussions which so often led to new research avenues that were critical for the success of my project. Their continuous support, patience, motivation, and immense knowledge have been inspirational.

My gratitude goes out to all past members of the lab who welcomed me with open arms and helped with the transition from the clinics to the lab, but specially to Janani Sivakumaran for her warm-hearted enthusiasm for science and for setting the example of high standards research, to Thomas Conlan for having the patience to teach me and help me when I started in the lab, to Cara Lomas for her cheerful companionship, and to Sophie Ward for all the help during the long days of experiments. I extend a warm thank you to everyone at the Institute of Immunity and Transplantation, from Prof Chakraverty and 
Dr Bennett's research group and beyond, for all the input, help, discussions and friendship.

I would also like to pay a special tribute to Prof João Forjaz Lacerda, Dr Maria João Costa and Dr Maria de Lurdes Guerra, my mentors during my Haematology residency, for their guidance, friendship and incentive to pursue a physician-scientist career.

Finally, I must thank my family: my parents and my sister, for always believing in me and encouraging me to go further, even when it meant moving to a different country; my parents-in-law, for their unconditional support and accepting to give Snoopy a new home until we can be re-joined; and most of all, my wife Rita, for her unfaltering love and for being by my side all the way through this long journey. 
VII. References 


\section{References}

1. Baron F, Storb R, Little MT. Hematopoietic cell transplantation: Five decades of progress. Arch Med Res 2003;34(6):528-44.

2. Little MT, Storb R. History of haematopoietic stem-cell transplantation. Nat Rev Cancer 2002, Mar;2(3):231-8.

3. Gratwohl A, Carreras E. Chapter 8 - principles of conditioning. In: Apperley J, Carreras E, Gluckman E, Masszi T, editors. ESH-EBMT Handbook on Haematopoietic Stem Cell Transplantation. European School of Haematology, Paris; 2012. p. 122-37.

4. Gyurkocza B, Sandmaier BM. Conditioning regimens for hematopoietic cell transplantation: One size does not fit all. Blood 2014, Jul 17;124(3):344-53.

5. Deeg HJ, Maris MB, Scott BL, Warren EH. Optimization of allogeneic transplant conditioning: Not the time for dogma. Leukemia 2006, Oct;20(10):1701-5.

6. Gluckman E. Chapter 6 - choice of the donor according to HLA typing and stem cell source. In: Apperley J, Carreras E, Gluckman E, Masszi T, editors. ESH-EBMT Handbook on Haematopoietic Stem Cell Transplantation. European School of Haematology, Paris; 2012. p. 90107.

7. Chervenick PA, Boggs DR. In vitro growth of granulocytic and mononuclear cell colonies from blood of normal individuals. Blood 1971, Feb;37(2):131-5.

8. Kurnick JE, Robison WA. Colony growth of human peripheral white blood cells in vitro. Blood 1971, Feb;37(2):136-41.

9. McCredie KB, Hersh EM, Freireich EJ. Cells capable of colony formation in the peripheral blood of man. Science 1971, Jan 22;171(3968):293-4.

10. Fliedner TM, Körbling M, Calvo W, Bruch C, Herbst E. Cryopreservation of blood mononuclear leukocytes and stem cells suspended in a large fluid 
volume. A preclinical model for a blood stem cell bank. Blut 1977, Sep 29;35(3):195-202.

11. Passweg JR, Baldomero H, Bader P, Bonini C, Cesaro S, Dreger P, et al. Hematopoietic stem cell transplantation in europe 2014: More than 40000 transplants annually. Bone Marrow Transplant 2016, Jun;51(6):786-92.

12. Prindull G, Prindull B, Meulen N. Haematopoietic stem cells (cfuc) in human cord blood. Acta Paediatr Scand 1978, Jul;67(4):413-6.

13. Gluckman E, Broxmeyer HA, Auerbach AD, Friedman HS, Douglas GW, Devergie $A$, et al. Hematopoietic reconstitution in a patient with fanconi's anemia by means of umbilical-cord blood from an hla-identical sibling. $\mathrm{N}$ Engl J Med 1989, Oct 26;321(17):1174-8.

14. Alshemmari S, Ameen R, Gaziev J. Haploidentical hematopoietic stemcell transplantation in adults. Bone Marrow Res 2011;2011:303487.

15. Martelli MF, Di lanni M, Ruggeri L, Falzetti F, Carotti A, Terenzi A, et al. HLA-haploidentical transplantation with regulatory and conventional t-cell adoptive immunotherapy prevents acute leukemia relapse. Blood 2014, Jul 24;124(4):638-44.

16. Schumm M, Lang $P$, Bethge $W$, Faul C, Feuchtinger T, Pfeiffer M, et al. Depletion of $\mathrm{t}$-cell receptor alpha/beta and CD19 positive cells from apheresis products with the clinimacs device. Cytotherapy 2013, Oct;15(10):1253-8.

17. Bertaina A, Merli P, Rutella S, Pagliara D, Bernardo ME, Masetti R, et al. HLA-haploidentical stem cell transplantation after removal of $\alpha \beta+T$ and $B$ cells in children with nonmalignant disorders. Blood 2014, Jul 31;124(5):822-6.

18. Pende D, Marcenaro S, Falco M, Martini S, Bernardo ME, Montagna D, et al. Anti-leukemia activity of alloreactive NK cells in KIR ligand-mismatched haploidentical HSCT for pediatric patients: Evaluation of the functional role of activating KIR and redefinition of inhibitory KIR specificity. Blood 2009, Mar 26;113(13):3119-29.

19. Roy D-C, Guerin M, Boumedine RS, Lachance S, Cohen S, Sauvageau $G$, et al. Reduction in incidence of severe infections by transplantation of high doses of haploidentical $T$ cells selectively depleted of alloreactive units. Blood 2011;118(21):3020-.

20. Sureda A, Bader P, Cesaro S, Dreger P, Duarte RF, Dufour C, et al. Indications for allo- and auto-sct for haematological diseases, solid 
tumours and immune disorders: Current practice in europe, 2015. Bone Marrow Transplant 2015, Aug;50(8):1037-56.

21. Thomas ED. A history of haemopoietic cell transplantation. $\mathrm{Br} \mathrm{J}$ Haematol 1999;105(2):330-9.

22. Jacobson LO, Marks EK, Robson MJ, Gaston EO, Zirkle RE. Effect of spleen protection on mortality following x-irradiation. J Lab Clin Med 1949;34:1538-43.

23. Jacobson LO, Simmons EL, Marks EK, Eldredge JH. Recovery from radiation injury. Science 1951, May 4;113(2940):510-1.

24. Lorenz E, Uphoff D, Reid TR, Shelton E. Modification of irradiation injury in mice and guinea pigs by bone marrow injections. J Natl Cancer Inst 1951, Aug;12(1):197-201.

25. Barnes DWH, Loutit JF. What is the recovery factor in spleen? Nucleonics 1954;12(5):68-71.

26. Main JM, Prehn RT. Successful skin homografts after the administration of high dosage $X$ radiation and homologous bone marrow. J Natl Cancer Inst 1955, Feb;15(4):1023-9.

27. Ford CE, Hamerton JL, Barnes DW, Loutit JF. Cytological identification of radiation-chimaeras. Nature 1956, Mar 10;177(4506):452-4.

28. Nowell PC, Cole LJ, Habermeyer JG, Roan PL. Growth and continued function of rat marrow cells in x-radiated mice. Cancer Res 1956, Mar;16(3):258-61.

29. Trentin JJ. Mortality and skin transplantability in x-irradiated mice receiving isologous, homologous or heterologous bone marrow. Proc Soc Exp Biol Med 1956;92(4):688-93.

30. Bekkum DWV. Radiation chimaeras. London: Logos Press; 1967.

31. van Bekkum DW, Vos O, Weyzen WW. [Homografts and heterografts of hemopoietic tissue in mice]. Rev Hematol 1956;11(5):477-85.

32. Santos GW, Owens $A H$. Allogeneic marrow transplants in cyclophosphamide treated mice. Transplant Proc 1969, Mar;1(1):44-6.

33. Billingham RE, Brent L. Quantitative studies on tissue transplantation immunity. IV. Induction of tolerance in newborn mice and studies on the phenomenon of runt disease. Philosophical Transactions of the Royal Society of London B: Biological Sciences 1959;242(694):439-77. 
34. Uphoff DE. Genetic factors influencing irradiation protection by bone marrow. I. The F1 hybrid effect. J Natl Cancer Inst 1957, Jul;19(1):12330 .

35. Lochte HL, Levy AS, Guenther DM, Thomas ED, Ferrebee JW. Prevention of delayed foreign marrow reaction in lethally irradiated mice by early administration of methotrexate. Nature 1962, Dec 15;196:1110-1.

36. Bortin MM. A compendium of reported human bone marrow transplants. Transplantation 1970, Jun;9(6):571-87.

37. Storb R, Thomas ED. Graft-versus-host disease in dog and man: The seattle experience. Immunol Rev 1985, Dec;88:215-38.

38. Amos DB, Gorer PA, Mikulska ZB. An analysis of an antigenic system in the mouse (the H-2 system). Proc R Soc Lond B Biol Sci 1955, Nov 29;144(916):369-80.

39. Epstein RB, Storb R, Ragde H, Thomas ED. Cytotoxic typing antisera for marrow grafting in littermate dogs. Transplantation 1968, Jan;6(1):45-58.

40. Storb R, Rudolph RH, Kolb HJ, Graham TC, Mickelson E, Erickson V, et al. Marrow grafts between dl-a-matched canine littermates. Transplantation 1973, Jan;15(1):92-100.

41. Storb R, Epstein RB, Graham TC, Thomas ED. Methotrexate regimens for control of graft-versus-host disease in dogs with allogeneic marrow grafts. Transplantation 1970, Mar;9(3):240-6.

42. Dausset J. [Iso-leuko-antibodies]. Acta Haematol 1958;20(1-4):156-66.

43. van Rood JJ. The detection of transplantation antigens in leukocytes. Semin Hematol 1968, Apr;5(2):187-214.

44. Vriesendorp HM, Heidt PJ. History of graft-versus-host disease. Exp Hematol 2016, Aug;44(8):674-88.

45. Gatti RA, Meuwissen HJ, Allen HD, Hong R, Good RA. Immunological reconstitution of sex-linked lymphopenic immunological deficiency. Lancet 1968, Dec 28;2(7583):1366-9.

46. Bach FH, Albertini RJ, Joo P, Anderson JL, Bortin MM. Bone-marrow transplantation in a patient with the wiskott-aldrich syndrome. Lancet 1968, Dec 28;2(7583):1364-6.

47. De Koning J, Van Bekkum DW, Dicke KA, Dooren LJ, Rádl J, Van Rood JJ. Transplantation of bone-marrow cells and fetal thymus in an infant with lymphopenic immunological deficiency. Lancet 1969, Jun 21;1(7608):1223-7. 
48. Bortin MM, Bach FH, van Bekkum DW, Good RA, van Rood JJ. 25th anniversary of the first successful allogeneic bone marrow transplants. Bone Marrow Transplant 1994, Aug;14(2):211-2.

49. Storb R, Buckner CD, Fefer A, Clift RA, Neiman PE, Gluksberg H, et al. Marrow transplantation in aplastic anemia. Transplant Proc 1974, Dec;6(4):355-8.

50. Thomas ED, Buckner CD, Banaji M, Clift RA, Fefer A, Flournoy N, et al. One hundred patients with acute leukemia treated by chemotherapy, total body irradiation, and allogeneic marrow transplantation. Blood 1977, Apr;49(4):511-33.

51. Storb R, Raff RF, Appelbaum FR, Deeg HJ, Fitzsimmons W, Graham TC, et al. FK-506 and methotrexate prevent graft-versus-host disease in dogs given 9.2 gy total body irradiation and marrow grafts from unrelated dog leukocyte antigen-nonidentical donors. Transplantation 1993, Oct;56(4):800-7.

52. Storb R, Deeg HJ, Whitehead J, Appelbaum F, Beatty P, Bensinger W, et al. Methotrexate and cyclosporine compared with cyclosporine alone for prophylaxis of acute graft versus host disease after marrow transplantation for leukemia. N Engl J Med 1986, Mar 20;314(12):729-35.

53. Ratanatharathorn V, Nash RA, Przepiorka D, Devine SM, Klein JL, Weisdorf $\mathrm{D}$, et al. Phase III study comparing methotrexate and tacrolimus (prograf, FK506) with methotrexate and cyclosporine for graft-versus-host disease prophylaxis after hla-identical sibling bone marrow transplantation. Blood 1998, Oct 1;92(7):2303-14.

54. Weiden PL, Flournoy N, Thomas ED, Prentice R, Fefer A, Buckner CD, Storb R. Antileukemic effect of graft-versus-host disease in human recipients of allogeneic-marrow grafts. N Engl J Med 1979, May 10;300(19):1068-73.

55. Weiden PL, Sullivan KM, Flournoy N, Storb R, Thomas ED. Antileukemic effect of chronic graft-versus-host disease: Contribution to improved survival after allogeneic marrow transplantation. N Engl J Med 1981, Jun 18;304(25):1529-33.

56. Sullivan KM, Weiden PL, Storb R, Witherspoon RP, Fefer A, Fisher L, et al. Influence of acute and chronic graft-versus-host disease on relapse and survival after bone marrow transplantation from hla-identical siblings as treatment of acute and chronic leukemia. Blood 1989, May $1 ; 73(6): 1720-8$. 
57. Freedman AS, Takvorian T, Anderson KC, Mauch P, Rabinowe SN, Blake $\mathrm{K}$, et al. Autologous bone marrow transplantation in b-cell non-hodgkin's lymphoma: Very low treatment-related mortality in 100 patients in sensitive relapse. J Clin Oncol 1990, May;8(5):784-91.

58. Philip T, Ladenstein R, Zucker JM, Pinkerton R, Bouffet E, Louis D, et al. Double megatherapy and autologous bone marrow transplantation for advanced neuroblastoma: The LMCE2 study. Br J Cancer 1993, Jan;67(1):119-27.

59. Santos GW, Tutschka PJ, Brookmeyer R, Saral R, Beschorner WE, Bias WB, et al. Marrow transplantation for acute nonlymphocytic leukemia after treatment with busulfan and cyclophosphamide. N Engl J Med 1983, Dec $1 ; 309(22): 1347-53$.

60. Oudshoorn M, van Leeuwen A, vd Zanden HG, van Rood JJ. Bone marrow donors worldwide: A successful exercise in international cooperation. Bone Marrow Transplant 1994, Jul;14(1):3-8.

61. Storb R, Deeg HJ. Failure of allogeneic canine marrow grafts after totalbody irradiation. Allogeneic "resistance" versus transfusion-induced sensitization. Transplantation 1986, Dec;42(6):571-80.

62. Erlich H. HLA DNA typing: Past, present, and future. Tissue Antigens 2012, Jul;80(1):1-11.

63. Saber W, Opie S, Rizzo JD, Zhang M-J, Horowitz MM, Schriber J. Outcomes after matched unrelated donor versus identical sibling hematopoietic cell transplantation in adults with acute myelogenous leukemia. Blood 2012, Apr 26;119(17):3908-16.

64. Körbling M, Drach J, Champlin RE, Engel H, Huynh L, Kleine HD, et al. Large-scale preparation of highly purified, frozen/thawed CD34+, HLADR- hematopoietic progenitor cells by sequential immunoadsorption (CEPRATE SC) and fluorescence-activated cell sorting: Implications for gene transduction and/or transplantation. Bone Marrow Transplant 1994, May;13(5):649-54.

65. McSweeney PA, Niederwieser D, Shizuru JA, Sandmaier BM, Molina AJ, Maloney DG, et al. Hematopoietic cell transplantation in older patients with hematologic malignancies: Replacing high-dose cytotoxic therapy with graft-versus-tumor effects. Blood 2001, Jun 1;97(11):3390-400.

66. Dreger P, Brand R, Hansz J, Milligan D, Corradini P, Finke J, et al. Treatment-related mortality and graft-versus-leukemia activity after 
allogeneic stem cell transplantation for chronic lymphocytic leukemia using intensity-reduced conditioning. Leukemia 2003, May;17(5):841-8.

67. Aoudjhane M, Labopin M, Gorin NC, Shimoni A, Ruutu T, Kolb H-J, et al. Comparative outcome of reduced intensity and myeloablative conditioning regimen in HLA identical sibling allogeneic haematopoietic stem cell transplantation for patients older than 50 years of age with acute myeloblastic leukaemia: A retrospective survey from the acute leukemia working party (ALWP) of the european group for blood and marrow transplantation (EBMT). Leukemia 2005, Dec;19(12):2304-12.

68. Mohty M, Labopin M, Volin L, Gratwohl A, Socié G, Esteve J, et al. Reduced-intensity versus conventional myeloablative conditioning allogeneic stem cell transplantation for patients with acute lymphoblastic leukemia: A retrospective study from the european group for blood and marrow transplantation. Blood 2010, Nov 25;116(22):4439-43.

69. Champlin RE, Passweg JR, Zhang MJ, Rowlings PA, Pelz CJ, Atkinson $\mathrm{KA}$, et al. T-cell depletion of bone marrow transplants for leukemia from donors other than hla-identical siblings: Advantage of t-cell antibodies with narrow specificities. Blood 2000, Jun 15;95(12):3996-4003.

70. Wagner JE, Thompson JS, Carter SL, Kernan NA, Unrelated Donor Marrow Transplantation Trial. Effect of graft-versus-host disease prophylaxis on 3-year disease-free survival in recipients of unrelated donor bone marrow (t-cell depletion trial): A multi-centre, randomised phase II-III trial. Lancet 2005;366(9487):733-41.

71. Schmid C, Labopin M, Nagler A, Bornhäuser M, Finke J, Fassas A, et al. Donor lymphocyte infusion in the treatment of first hematological relapse after allogeneic stem-cell transplantation in adults with acute myeloid leukemia: A retrospective risk factors analysis and comparison with other strategies by the EBMT acute leukemia working party. J Clin Oncol 2007, Nov 1;25(31):4938-45.

72. Sathe A, Ortega SB, Mundy DI, Collins RH, Karandikar NJ. In vitro methotrexate as a practical approach to selective allodepletion. Biol Blood Marrow Transplant 2007, Jun;13(6):644-54.

73. Anderson BE, McNiff J, Yan J, Doyle H, Mamula M, Shlomchik MJ, Shlomchik WD. Memory CD4+ T cells do not induce graft-versus-host disease. J Clin Invest 2003, Jul;112(1):101-8.

74. Verfuerth S, Sousa PSE, Beloki L, Murray M, Peters MD, Mackinnon S, et al. Generation of memory $T$ cells for adoptive transfer using clinical-grade 
anti-cd62I magnetic beads. Bone Marrow Transplant 2015, Jun 15;50(10):1358-64.

75. Chakraverty R, Flutter B, Fallah-Arani F, Eom H-S, Means T, Andreola G, et al. The host environment regulates the function of CD8+ graft-versushost-reactive effector cells. J Immunol 2008, Nov 15;181(10):6820-8.

76. Cohen JL, Trenado A, Vasey D, Klatzmann D, Salomon BL. CD4(+)CD25(+) immunoregulatory T cells: New therapeutics for graftversus-host disease. J Exp Med 2002, Aug 5;196(3):401-6.

77. Rojas JM, Knight K, Wang L, Clark RE. Clinical evaluation of BCR-ABL peptide immunisation in chronic myeloid leukaemia: Results of the EPIC study. Leukemia 2007, Nov;21(11):2287-95.

78. Manzo T, Michelini RH, Sturmheit T, Basso V, Bellone M, Mondino A. Tumor-targeting vaccination instructs graft-vs.-tumor immune responses. Oncoimmunology 2013, Oct 1;2(10):e25996.

79. Tsimberidou A-M, Giles FJ, Estey E, O'Brien S, Keating MJ, Kantarjian $\mathrm{HM}$. The role of gemtuzumab ozogamicin in acute leukaemia therapy. $\mathrm{Br}$ J Haematol 2006, Feb;132(4):398-409.

80. Marin V, Dander E, Biagi E, Introna M, Fazio G, Biondi A, D'Amico G. Characterization of in vitro migratory properties of anti-cd19 chimeric receptor-redirected CIK cells for their potential use in B-ALL immunotherapy. Exp Hematol 2006, Sep;34(9):1219-29.

81. Ferrara JLM, Levine JE, Reddy P, Holler E. Graft-versus-host disease. Lancet 2009, May 2;373(9674):1550-61.

82. Pavletic SZ, Fowler DH. Are we making progress in GVHD prophylaxis and treatment? Hematology Am Soc Hematol Educ Program 2012;2012:251-64.

83. Filipovich AH, Weisdorf D, Pavletic S, Socie G, Wingard JR, Lee SJ, et al. National institutes of health consensus development project on criteria for clinical trials in chronic graft-versus-host disease: I. Diagnosis and staging working group report. Biol Blood Marrow Transplant 2005, Dec;11(12):945-56.

84. Jacobsohn DA. Acute graft-versus-host disease in children. Bone Marrow Transplant 2008, Jan;41(2):215-21.

85. Ball LM, Egeler RM, EBMT Paediatric Working Party. Acute gvhd: Pathogenesis and classification. Bone Marrow Transplant 2008, Jun;41 Suppl 2:S58-64. 
86. Lee SJ, Vogelsang G, Flowers MED. Chronic graft-versus-host disease. Biol Blood Marrow Transplant 2003, Apr;9(4):215-33.

87. Barnes DW, Loutit JF. Treatment of murine leukaemia with x-rays and homologous bone marrow. II. Br J Haematol 1957, Jul;3(3):241-52.

88. Billingham RE. The biology of graft-versus-host reactions. Harvey Lect 1966;62:21-78.

89. Korngold R, Sprent J. Lethal graft-versus-host disease after bone marrow transplantation across minor histocompatibility barriers in mice. Prevention by removing mature T cells from marrow. J Exp Med 1978, Dec 1;148(6):1687-98.

90. Socie G, Blazar BR. Acute graft-versus-host disease: From the bench to the bedside. Blood 2009, Nov 12;114(20):4327-36.

91. Welniak LA, Blazar BR, Murphy WJ. Immunobiology of allogeneic hematopoietic stem cell transplantation. Annu Rev Immunol 2007;25:13970.

92. Nestel FP, Price KS, Seemayer TA, Lapp WS. Macrophage priming and lipopolysaccharide-triggered release of tumor necrosis factor alpha during graft-versus-host disease. J Exp Med 1992, Feb 1;175(2):405-13.

93. Xun CQ, Thompson JS, Jennings CD, Brown SA, Widmer MB. Effect of total body irradiation, busulfan-cyclophosphamide, or cyclophosphamide conditioning on inflammatory cytokine release and development of acute and chronic graft-versus-host disease in h-2-incompatible transplanted SCID mice. Blood 1994, Apr 15;83(8):2360-7.

94. Hill GR, Crawford JM, Cooke KR, Brinson YS, Pan L, Ferrara JL. Total body irradiation and acute graft-versus-host disease: The role of gastrointestinal damage and inflammatory cytokines. Blood 1997, Oct 15;90(8):3204-13.

95. Markey KA, MacDonald KPA, Hill GR. The biology of graft-versus-host disease: Experimental systems instructing clinical practice. Blood 2014, Jul 17;124(3):354-62.

96. Cooke KR, Olkiewicz K, Erickson N, Ferrara JLM. The role of endotoxin and the innate immune response in the pathophysiology of acute graft versus host disease. J Endotoxin Res 2002;8(6):441-8.

97. Calcaterra C, Sfondrini L, Rossini A, Sommariva M, Rumio C, Ménard S, Balsari A. Critical role of TLR9 in acute graft-versus-host disease. J Immunol 2008, Nov 1;181(9):6132-9. 
98. Panoskaltsis-Mortari A, Lacey DL, Vallera DA, Blazar BR. Keratinocyte growth factor administered before conditioning ameliorates graft-versushost disease after allogeneic bone marrow transplantation in mice. Blood 1998, Nov 15;92(10):3960-7.

99. Hill GR, Cooke KR, Teshima T, Crawford JM, Keith JC, Brinson YS, et al. Interleukin-11 promotes $\mathrm{T}$ cell polarization and prevents acute graftversus-host disease after allogeneic bone marrow transplantation. J Clin Invest 1998, Jul 1;102(1):115-23.

100. Mathewson ND, Jenq $R$, Mathew AV, Koenigsknecht M, Hanash A, Toubai $\mathrm{T}$, et al. Gut microbiome-derived metabolites modulate intestinal epithelial cell damage and mitigate graft-versus-host disease. Nat Immunol 2016, May;17(5):505-13.

101. Shono Y, Docampo MD, Peled JU, Perobelli SM, Jenq RR. Intestinal microbiota-related effects on graft-versus-host disease. Int $\mathrm{J}$ Hematol 2015, May;101(5):428-37.

102. Wang W, Xu S, Ren Z, Jiang J, Zheng S. Gut microbiota and allogeneic transplantation. J Transl Med 2015;13:275.

103. Zama D, Biagi E, Masetti R, Gasperini P, Prete A, Candela M, et al. Gut microbiota and hematopoietic stem cell transplantation: Where do we stand? Bone Marrow Transplant 2016, Jun 27.

104. Chakraverty R, Côté D, Buchli J, Cotter P, Hsu R, Zhao G, et al. An inflammatory checkpoint regulates recruitment of graft-versus-host reactive T cells to peripheral tissues. J Exp Med 2006, Aug 7;203(8):202131.

105. Wilhelm K, Ganesan J, Müller T, Dürr C, Grimm M, Beilhack A, et al. Graftversus-host disease is enhanced by extracellular ATP activating P2X7R. Nat Med 2010, Dec;16(12):1434-8.

106. Clift RA, Buckner CD, Appelbaum FR, Bryant E, Bearman SI, Petersen $\mathrm{FB}$, et al. Allogeneic marrow transplantation in patients with chronic myeloid leukemia in the chronic phase: A randomized trial of two irradiation regimens. Blood 1991, Apr 15;77(8):1660-5.

107. Socié G, Clift RA, Blaise D, Devergie A, Ringden O, Martin PJ, et al. Busulfan plus cyclophosphamide compared with total-body irradiation plus cyclophosphamide before marrow transplantation for myeloid leukemia: Long-term follow-up of 4 randomized studies. Blood 2001, Dec 15;98(13):3569-74. 
108. Mielcarek M, Martin PJ, Leisenring W, Flowers MED, Maloney DG, Sandmaier BM, et al. Graft-versus-host disease after nonmyeloablative versus conventional hematopoietic stem cell transplantation. Blood 2003, Jul 15;102(2):756-62.

109. Appleman LJ, Boussiotis VA. T cell anergy and costimulation. Immunol Rev 2003, Apr;192:161-80.

110. Blazar BR, Taylor PA, Linsley PS, Vallera DA. In vivo blockade of CD28/CTLA4: B7/BB1 interaction with ctla4-ig reduces lethal murine graftversus-host disease across the major histocompatibility complex barrier in mice. Blood 1994, Jun 15;83(12):3815-25.

111. Buchan SL, Manzo T, Flutter B, Rogel A, Edwards N, Zhang L, et al. OX40- and cd27-mediated costimulation synergizes with anti-pd-I1 blockade by forcing exhausted CD8+ T cells to exit quiescence. J Immunol 2015, Jan 1;194(1):125-33.

112. Shlomchik WD, Couzens MS, Tang CB, McNiff J, Robert ME, Liu J, et al. Prevention of graft versus host disease by inactivation of host antigenpresenting cells. Science 1999, Jul 16;285(5426):412-5.

113. Matte CC, Liu J, Cormier J, Anderson BE, Athanasiadis I, Jain D, et al. Donor apcs are required for maximal GVHD but not for GVL. Nat Med 2004, Sep;10(9):987-92.

114. Lechler R, Ng WF, Steinman RM. Dendritic cells in transplantation--friend or foe? Immunity 2001, Apr;14(4):357-68.

115. Sayegh $\mathrm{MH}$, Carpenter $\mathrm{CB}$. Role of indirect allorecognition in allograft rejection. Int Rev Immunol 1996;13(3):221-9.

116. Wang X, Li H, Matte-Martone C, Cui W, Li N, Tan HS, et al. Mechanisms of antigen presentation to $\mathrm{T}$ cells in murine graft-versus-host disease: Cross-presentation and the appearance of cross-presentation. Blood 2011, Dec 8;118(24):6426-37.

117. Koyama M, Kuns RD, Olver SD, Raffelt NC, Wilson YA, Don ALJ, et al. Recipient nonhematopoietic antigen-presenting cells are sufficient to induce lethal acute graft-versus-host disease. Nat Med 2012, Jan;18(1):135-42.

118. Toubai T, Tawara I, Sun Y, Liu C, Nieves E, Evers R, et al. Induction of acute GVHD by sex-mismatched $\mathrm{H}-\mathrm{Y}$ antigens in the absence of functional radiosensitive host hematopoietic-derived antigen-presenting cells. Blood 2012, Apr 19;119(16):3844-53. 
119. Zhu J, Yamane H, Paul WE. Differentiation of effector CD4 $T$ cell populations $\left(^{*}\right)$. Annu Rev Immunol 2010;28:445-89.

120. Sung AD, Chao NJ. Concise review: Acute graft-versus-host disease: Immunobiology, prevention, and treatment. Stem Cells Transl Med 2013, Jan;2(1):25-32.

121. Reddy P. Pathophysiology of acute graft-versus-host disease. Hematol Oncol 2003, Dec;21(4):149-61.

122. Lu Y, Waller EK. Dichotomous role of interferon-gamma in allogeneic bone marrow transplant. Biol Blood Marrow Transplant 2009, Nov;15(11):1347-53.

123. Fowler DH, Gress RE. Th2 and tc2 cells in the regulation of GVHD, GVL, and graft rejection: Considerations for the allogeneic transplantation therapy of leukemia and lymphoma. Leuk Lymphoma 2000, Jul;38(34):221-34.

124. Reddy P, Teshima T, Hildebrandt G, Williams DL, Liu C, Cooke KR, Ferrara JLM. Pretreatment of donors with interleukin-18 attenuates acute graft-versus-host disease via STAT6 and preserves graft-versusleukemia effects. Blood 2003, Apr 1;101(7):2877-85.

125. Fowler DH, Kurasawa K, Smith R, Eckhaus MA, Gress RE. Donor cd4enriched cells of th2 cytokine phenotype regulate graft-versus-host disease without impairing allogeneic engraftment in sublethally irradiated mice. Blood 1994, Nov 15;84(10):3540-9.

126. Fowler DH, Kurasawa K, Husebekk A, Cohen PA, Gress RE. Cells of th2 cytokine phenotype prevent Ips-induced lethality during murine graftversus-host reaction. Regulation of cytokines and CD8+ lymphoid engraftment. J Immunol 1994, Feb 1;152(3):1004-13.

127. Krenger W, Cooke KR, Crawford JM, Sonis ST, Simmons R, Pan L, et al. Transplantation of polarized type 2 donor T cells reduces mortality caused by experimental graft-versus-host disease. Transplantation 1996, Nov 15;62(9):1278-85.

128. Nakae S, Nambu A, Sudo K, Iwakura Y. Suppression of immune induction of collagen-induced arthritis in il-17-deficient mice. J Immunol 2003, Dec 1;171(11):6173-7.

129. Komiyama $Y$, Nakae S, Matsuki T, Nambu A, Ishigame $H$, Kakuta $S$, et al. IL-17 plays an important role in the development of experimental autoimmune encephalomyelitis. J Immunol 2006, Jul 1;177(1):566-73. 
130. Di Cesare A, Di Meglio P, Nestle FO. The IL-23/th17 axis in the immunopathogenesis of psoriasis. J Invest Dermatol 2009, Jun;129(6):1339-50.

131. Nograles KE, Zaba LC, Guttman-Yassky E, Fuentes-Duculan J, SuárezFariñas M, Cardinale I, et al. Th17 cytokines interleukin (IL)-17 and IL-22 modulate distinct inflammatory and keratinocyte-response pathways. $\mathrm{Br} \mathrm{J}$ Dermatol 2008, Nov;159(5):1092-102.

132. Sawa S, Lochner M, Satoh-Takayama N, Dulauroy S, Bérard $M$, Kleinschek $\mathrm{M}$, et al. RORyt+ innate lymphoid cells regulate intestinal homeostasis by integrating negative signals from the symbiotic microbiota. Nat Immunol 2011, Apr;12(4):320-6.

133. Mielke LA, Jones SA, Raverdeau M, Higgs R, Stefanska A, Groom JR, et al. Retinoic acid expression associates with enhanced IL-22 production by $y \delta T$ cells and innate lymphoid cells and attenuation of intestinal inflammation. J Exp Med 2013, Jun 3;210(6):1117-24.

134. Hanash AM, Dudakov JA, Hua G, O'Connor MH, Young LF, Singer NV, et al. Interleukin-22 protects intestinal stem cells from immune-mediated tissue damage and regulates sensitivity to graft versus host disease. Immunity 2012, Aug 24;37(2):339-50.

135. Panoskaltsis-Mortari A, Price A, Hermanson JR, Taras E, Lees C, Serody JS, Blazar BR. In vivo imaging of graft-versus-host-disease in mice. Blood 2004, May 1;103(9):3590-8.

136. Wysocki CA, Panoskaltsis-Mortari A, Blazar BR, Serody JS. Leukocyte migration and graft-versus-host disease. Blood 2005, Jun 1;105(11):4191-9.

137. Cyster JG, Schwab SR. Sphingosine-1-phosphate and lymphocyte egress from lymphoid organs. Annu Rev Immunol 2012;30:69-94.

138. Kim Y-M, Sachs T, Asavaroengchai W, Bronson R, Sykes M. Graftversus-host disease can be separated from graft-versus-lymphoma effects by control of lymphocyte trafficking with FTY720. J Clin Invest 2003, Mar;111(5):659-69.

139. Kim CH, Nagata K, Butcher EC. Dendritic cells support sequential reprogramming of chemoattractant receptor profiles during naive to effector T cell differentiation. J Immunol 2003, Jul 1;171(1):152-8.

140. New JY, Li B, Koh WP, Ng HK, Tan SY, Yap EH, et al. T cell infiltration and chemokine expression: Relevance to the disease localization in 
murine graft-versus-host disease. Bone Marrow Transplant 2002, Jun;29(12):979-86.

141. Murai M, Yoneyama H, Harada A, Yi Z, Vestergaard C, Guo B, et al. Active participation of CCR5(+)CD8(+) T lymphocytes in the pathogenesis of liver injury in graft-versus-host disease. J Clin Invest 1999, Jul;104(1):4957.

142. Cyster JG. Chemokines, sphingosine-1-phosphate, and cell migration in secondary lymphoid organs. Annu Rev Immunol 2005;23:127-59.

143. Waldman E, Lu SX, Hubbard VM, Kochman AA, Eng JM, Terwey TH, et al. Absence of beta7 integrin results in less graft-versus-host disease because of decreased homing of alloreactive $T$ cells to intestine. Blood 2006, Feb 15;107(4):1703-11.

144. Petrovic A, Alpdogan O, Willis LM, Eng JM, Greenberg AS, Kappel BJ, et al. LPAM (alpha 4 beta 7 integrin) is an important homing integrin on alloreactive $\mathrm{T}$ cells in the development of intestinal graft-versus-host disease. Blood 2004, Feb 15;103(4):1542-7.

145. Baker M, Podack ER, Levy RB. Fas and perforin cytotoxic pathways are not the major effector mechanisms in allogeneic resistance to bone marrow. Ann N Y Acad Sci 1995, Dec 29;770:368-9.

146. Baker MB, Altman NH, Podack ER, Levy RB. The role of cell-mediated cytotoxicity in acute GVHD after mhc-matched allogeneic bone marrow transplantation in mice. J Exp Med 1996, Jun 1;183(6):2645-56.

147. Das H, Imoto S, Murayama $\mathrm{T}$, Kajimoto $\mathrm{K}$, Sugimoto $\mathrm{T}$, Isobe $\mathrm{T}$, et al. Levels of soluble fasl and fasl gene expression during the development of graft-versus-host disease in dlt-treated patients. Br J Haematol 1999, Mar;104(4):795-800.

148. Liem LM, van Lopik T, van Nieuwenhuijze AE, van Houwelingen HC, Aarden L, Goulmy E. Soluble fas levels in sera of bone marrow transplantation recipients are increased during acute graft-versus-host disease but not during infections. Blood 1998, Feb 15;91(4):1464-8.

149. van den Brink MRM, Burakoff SJ. Cytolytic pathways in haematopoietic stem-cell transplantation. Nat Rev Immunol 2002, Apr;2(4):273-81.

150. Jaksch M, Mattsson J. The pathophysiology of acute graft-versus-host disease. Scand J Immunol 2005, May;61(5):398-409.

151. Chopra M, Brandl A, Siegmund D, Mottok A, Schäfer V, Biehl M, et al. Blocking tweak-fn14 interaction inhibits hematopoietic stem cell 
transplantation-induced intestinal cell death and reduces GVHD. Blood 2015, Jul 23;126(4):437-44.

152. Ghosh A, Dogan Y, Moroz M, Holland AM, Yim NL, Rao UK, et al. Adoptively transferred TRAIL+ T cells suppress GVHD and augment antitumor activity. J Clin Invest 2013, Jun;123(6):2654-62.

153. Tamada K, Tamura H, Flies D, Fu Y-X, Celis E, Pease LR, et al. Blockade of LIGHT/ltbeta and CD40 signaling induces allospecific T cell anergy, preventing graft-versus-host disease. J Clin Invest 2002, Feb;109(4):54957.

154. Reddy P, Ferrara JLM. Mouse models of graft-versus-host disease. In: Mathis D, Ritz J, editors. StemBook. Cambridge (MA): The Stem Cell Research Community,; 2008.

155. Villa NY, Rahman MM, McFadden G, Cogle CR. Therapeutics for graftversus-host disease: From conventional therapies to novel virotherapeutic strategies. Viruses 2016, Mar;8(3):85.

156. Couriel D, Saliba R, Hicks K, Ippoliti C, de Lima M, Hosing C, et al. Tumor necrosis factor-alpha blockade for the treatment of acute GVHD. Blood 2004, Aug 1;104(3):649-54.

157. Levine JE, Paczesny S, Mineishi S, Braun T, Choi SW, Hutchinson RJ, et al. Etanercept plus methylprednisolone as initial therapy for acute graftversus-host disease. Blood 2008, Feb 15;111(4):2470-5.

158. Alousi AM, Weisdorf DJ, Logan BR, Bolaños-Meade J, Carter S, Difronzo $\mathrm{N}$, et al. Etanercept, mycophenolate, denileukin, or pentostatin plus corticosteroids for acute graft-versus-host disease: A randomized phase 2 trial from the blood and marrow transplant clinical trials network. Blood 2009, Jul 16;114(3):511-7.

159. Antin JH, Ferrara JL. Cytokine dysregulation and acute graft-versus-host disease. Blood 1992, Dec 15;80(12):2964-8.

160. Abhyankar S, Gilliland DG, Ferrara JL. Interleukin-1 is a critical effector molecule during cytokine dysregulation in graft versus host disease to minor histocompatibility antigens. Transplantation 1993, Dec;56(6):151823.

161. Hill GR, Teshima T, Gerbitz A, Pan L, Cooke KR, Brinson YS, et al. Differential roles of IL-1 and tnf-alpha on graft-versus-host disease and graft versus leukemia. J Clin Invest 1999, Aug;104(4):459-67. 
162. Park M-J, Lee SH, Lee S-H, Lee E-J, Kim E-K, Choi JY, Cho M-L. IL-1 receptor blockade alleviates graft-versus-host disease through downregulation of an interleukin-1 $\beta$-dependent glycolytic pathway in th17 cells. Mediators Inflamm 2015;2015:631384.

163. Antin JH, Weisdorf D, Neuberg D, Nicklow R, Clouthier S, Lee SJ, et al. Interleukin-1 blockade does not prevent acute graft-versus-host disease: Results of a randomized, double-blind, placebo-controlled trial of interleukin-1 receptor antagonist in allogeneic bone marrow transplantation. Blood 2002, Nov 15;100(10):3479-82.

164. Yang Y-G, Wang H, Asavaroengchai W, Dey BR. Role of interferongamma in GVHD and GVL. Cell Mol Immunol 2005, Oct;2(5):323-9.

165. Ferrara JLM, Reddy P. Pathophysiology of graft-versus-host disease. Semin Hematol 2006, Jan;43(1):3-10.

166. Ellison CA, Natuik SA, Mclntosh AR, Scully SA, Danilenko DM, Gartner JG. The role of interferon-gamma, nitric oxide and lipopolysaccharide in intestinal graft-versus-host disease developing in f1-hybrid mice. Immunology 2003, Jul;109(3):440-9.

167. Krenger W, Falzarano G, Delmonte J, Snyder KM, Byon JC, Ferrara JL. Interferon-gamma suppresses t-cell proliferation to mitogen via the nitric oxide pathway during experimental acute graft-versus-host disease. Blood 1996, Aug 1;88(3):1113-21.

168. Chen X, Das R, Komorowski R, Beres A, Hessner MJ, Mihara M, Drobyski WR. Blockade of interleukin-6 signaling augments regulatory t-cell reconstitution and attenuates the severity of graft-versus-host disease. Blood 2009, Jul 23;114(4):891-900.

169. Tawara I, Koyama M, Liu C, Toubai T, Thomas D, Evers R, et al. Interleukin-6 modulates graft-versus-host responses after experimental allogeneic bone marrow transplantation. Clin Cancer Res 2011, Jan $1 ; 17(1): 77-88$.

170. Das R, Chen X, Komorowski R, Hessner MJ, Drobyski WR. Interleukin-23 secretion by donor antigen-presenting cells is critical for organ-specific pathology in graft-versus-host disease. Blood 2009, Mar 5;113(10):235262.

171. Das R, Komorowski R, Hessner MJ, Subramanian H, Huettner CS, Cua $D$, Drobyski WR. Blockade of interleukin-23 signaling results in targeted protection of the colon and allows for separation of graft-versus-host and graft-versus-leukemia responses. Blood 2010, Jun 24;115(25):5249-58. 
172. Fulton LM, Carlson MJ, Coghill JM, Ott LE, West ML, Panoskaltsis-Mortari $A$, et al. Attenuation of acute graft-versus-host disease in the absence of the transcription factor roryt. J Immunol 2012, Aug 15;189(4):1765-72.

173. Liu Y, Cai Y, Dai L, Chen G, Ma X, Wang Y, et al. The expression of th17associated cytokines in human acute graft-versus-host disease. Biol Blood Marrow Transplant 2013, Oct;19(10):1421-9.

174. Muranski $P$, Restifo NP. Essentials of th17 cell commitment and plasticity. Blood 2013, Mar 28;121(13):2402-14.

175. Deeg HJ, Storb R, Shulman HM, Weiden PL, Graham TC, Thomas ED. Engraftment of dla-nonidentical unrelated canine marrow after high-dose fractionated total body irradiation. Transplantation 1982, Apr;33(4):443-6.

176. Storb R, Yu C, Wagner JL, Deeg HJ, Nash RA, Kiem HP, et al. Stable mixed hematopoietic chimerism in dla-identical littermate dogs given sublethal total body irradiation before and pharmacological immunosuppression after marrow transplantation. Blood 1997, Apr 15;89(8):3048-54.

177. Storb R, Kolb HJ, Graham TC, Kolb H, Weiden PL, Thomas ED. Treatment of established graft-versus-host disease in dogs by antithymocyte serum or prednisone. Blood 1973, Oct;42(4):601-9.

178. Atkinson K, Shulman HM, Deeg HJ, Weiden PL, Graham TC, Thomas ED, Storb R. Acute and chronic graft-versus-host disease in dogs given hemopoietic grafts from dla-nonidentical littermates. Two distinct syndromes. Am J Pathol 1982, Aug;108(2):196-205.

179. Stolfi JL, Pai C-CS, Murphy WJ. Preclinical modeling of hematopoietic stem cell transplantation - advantages and limitations. FEBS J 2016, May;283(9):1595-606.

180. Blazar BR, Murphy WJ, Abedi M. Advances in graft-versus-host disease biology and therapy. Nat Rev Immunol 2012, Jun;12(6):443-58.

181. Ericsson AC, Davis JW, Spollen W, Bivens N, Givan S, Hagan CE, et al. Effects of vendor and genetic background on the composition of the fecal microbiota of inbred mice. PLoS One 2015;10(2):e0116704.

182. Chang H-YS, Mitzner W, Watson J. Variation in airway responsiveness of male C57BL/6 mice from 5 vendors. J Am Assoc Lab Anim Sci 2012, Jul;51(4):401-6.

183. Casellas J. Inbred mouse strains and genetic stability: A review. Animal 2011, Jan;5(1):1-7. 
184. Blazar BR, Lees CJ, Martin PJ, Noelle RJ, Kwon B, Murphy W, Taylor PA. Host $T$ cells resist graft-versus-host disease mediated by donor leukocyte infusions. J Immunol 2000, Nov 1;165(9):4901-9.

185. Via CS, Sharrow SO, Shearer GM. Role of cytotoxic T lymphocytes in the prevention of lupus-like disease occurring in a murine model of graft-vshost disease. J Immunol 1987, Sep 15;139(6):1840-9.

186. Bachar-Lustig E, Li HW, Gur H, Krauthgamer R, Marcus H, Reisner Y. Induction of donor-type chimerism and transplantation tolerance across major histocompatibility barriers in sublethally irradiated mice by sca$1(+)$ lin(-) bone marrow progenitor cells: Synergism with non-alloreactive (host $x$ donor) $F(1)$ T cells. Blood 1999, Nov 1;94(9):3212-21.

187. Holler E, Rogler G, Brenmoehl J, Hahn J, Herfarth H, Greinix H, et al. Prognostic significance of NOD2/CARD15 variants in hla-identical sibling hematopoietic stem cell transplantation: Effect on long-term outcome is confirmed in 2 independent cohorts and may be modulated by the type of gastrointestinal decontamination. Blood 2006, May 15;107(10):4189-93.

188. Holler E, Butzhammer P, Schmid K, Hundsrucker C, Koestler J, Peter K, et al. Metagenomic analysis of the stool microbiome in patients receiving allogeneic stem cell transplantation: Loss of diversity is associated with use of systemic antibiotics and more pronounced in gastrointestinal graftversus-host disease. Biol Blood Marrow Transplant 2014, May;20(5):6405 .

189. Ordemann R, Hutchinson R, Friedman J, Burakoff SJ, Reddy P, Duffner $U$, et al. Enhanced allostimulatory activity of host antigen-presenting cells in old mice intensifies acute graft-versus-host disease. J Clin Invest 2002, May;109(9):1249-56.

190. Gupta V, Eapen M, Brazauskas R, Carreras J, Aljurf M, Gale RP, et al. Impact of age on outcomes after bone marrow transplantation for acquired aplastic anemia using hla-matched sibling donors. Haematologica 2010, Dec;95(12):2119-25.

191. Schroeder MA, DiPersio JF. Mouse models of graft-versus-host disease: Advances and limitations. Dis Model Mech 2011, May;4(3):318-33.

192. Teshima T, Ordemann R, Reddy P, Gagin S, Liu C, Cooke KR, Ferrara JLM. Acute graft-versus-host disease does not require alloantigen expression on host epithelium. Nat Med 2002, Jun;8(6):575-81. 
193. Reddy P, Negrin R, Hill GR. Mouse models of bone marrow transplantation. Biol Blood Marrow Transplant 2008, Jan;14(1 Suppl 1):129-35.

194. Korngold R, Sprent J. Variable capacity of L3T4+ T cells to cause lethal graft-versus-host disease across minor histocompatibility barriers in mice. J Exp Med 1987, Jun 1;165(6):1552-64.

195. Chao NJ. Minors come of age: Minor histocompatibility antigens and graftversus-host disease. Biol Blood Marrow Transplant 2004, Apr;10(4):21523.

196. Eden PA, Christianson GJ, Fontaine P, Wettstein PJ, Perreault C, Roopenian DC. Biochemical and immunogenetic analysis of an immunodominant peptide (b6dom1) encoded by the classical $\mathrm{H} 7$ minor histocompatibility locus. J Immunol 1999, Apr 15;162(8):4502-10.

197. Choi EY, Christianson GJ, Yoshimura Y, Jung N, Sproule TJ, Malarkannan S, et al. Real-time t-cell profiling identifies $\mathrm{H} 60$ as a major minor histocompatibility antigen in murine graft-versus-host disease. Blood 2002, Dec 15;100(13):4259-65.

198. Yang J, Jaramillo A, Liu W, Olack B, Yoshimura Y, Joyce S, et al. Chronic rejection of murine cardiac allografts discordant at the $\mathrm{H} 13$ minor histocompatibility antigen correlates with the generation of the h13specific CD8+ cytotoxic T cells. Transplantation 2003, Jul 15;76(1):84-91.

199. Berger M, Wettstein PJ, Korngold R. T cell subsets involved in lethal graftversus-host disease directed to immunodominant minor histocompatibility antigens. Transplantation 1994, Apr 15;57(7):1095-102.

200. Fontaine P, Roy-Proulx G, Knafo L, Baron C, Roy DC, Perreault C. Adoptive transfer of minor histocompatibility antigen-specific $T$ lymphocytes eradicates leukemia cells without causing graft-versus-host disease. Nat Med 2001, Jul;7(7):789-94.

201. Reddy P, Socié G, Cutler C, Weisdorf D. GVHD prevention: An ounce is better than a pound. Biol Blood Marrow Transplant 2012, Jan;18(1 Suppl):S17-26.

202. Griem P, Wallny HJ, Falk K, Rötzschke O, Arnold B, Schönrich G, et al. Uneven tissue distribution of minor histocompatibility proteins versus peptides is caused by MHC expression. Cell 1991, May 17;65(4):633-40.

203. Zhang P, Wu J, Deoliveira D, Chao NJ, Chen BJ. Allospecific CD4(+) effector memory $\mathrm{T}$ cells do not induce graft-versus-host disease in mice. Biol Blood Marrow Transplant 2012, Oct;18(10):1488-99. 
204. Hartwig UF, Robbers M, Wickenhauser C, Huber C. Murine acute graftversus-host disease can be prevented by depletion of alloreactive $T$ lymphocytes using activation-induced cell death. Blood 2002, Apr 15;99(8):3041-9.

205. Shibaki A, Sato A, Vogel JC, Miyagawa F, Katz SI. Induction of gvhd-like skin disease by passively transferred CD8(+) t-cell receptor transgenic $T$ cells into keratin 14-ovalbumin transgenic mice. J Invest Dermatol 2004, Jul;123(1):109-15.

206. Randolph SSB, Gooley TA, Warren EH, Appelbaum FR, Riddell SR. Female donors contribute to a selective graft-versus-leukemia effect in male recipients of hla-matched, related hematopoietic stem cell transplants. Blood 2004, Jan 1;103(1):347-52.

207. Zorn E, Miklos DB, Floyd BH, Mattes-Ritz A, Guo L, Soiffer RJ, et al. Minor histocompatibility antigen DBY elicits a coordinated $B$ and $T$ cell response after allogeneic stem cell transplantation. J Exp Med 2004, Apr 19;199(8):1133-42.

208. Vogt MHJ, van den Muijsenberg JW, Goulmy E, Spierings E, Kluck P, Kester MG, et al. The DBY gene codes for an hla-dq5-restricted human male-specific minor histocompatibility antigen involved in graft-versushost disease. Blood 2002, Apr 15;99(8):3027-32.

209. Vogt MH, Goulmy E, Kloosterboer FM, Blokland E, de Paus RA, Willemze $\mathrm{R}$, Falkenburg JH. UTY gene codes for an hla-b60-restricted human malespecific minor histocompatibility antigen involved in stem cell graft rejection: Characterization of the critical polymorphic amino acid residues for t-cell recognition. Blood 2000, Nov 1;96(9):3126-32.

210. Flutter B, Edwards N, Fallah-Arani F, Henderson S, Chai J-G, Sivakumaran $S$, et al. Nonhematopoietic antigen blocks memory programming of alloreactive CD8+ $\mathrm{T}$ cells and drives their eventual exhaustion in mouse models of bone marrow transplantation<br $>$. J Clin Invest 2010, Nov;120(11):3855-68.

211. Capitini CM, Nasholm NM, Duncan BB, Guimond M, Fry TJ. Graft-versushost disease impairs vaccine responses through decreased CD4+ and CD8 $+\mathrm{T}$ cell proliferation and increased perforin-mediated CD8 $+\mathrm{T}$ cell apoptosis. J Immunol 2013, Feb 1;190(3):1351-9.

212. Murai M, Yoneyama $H$, Ezaki T, Suematsu M, Terashima $Y$, Harada A, et al. Peyer's patch is the essential site in initiating murine acute and lethal graft-versus-host reaction. Nat Immunol 2003, Feb;4(2):154-60. 
213. Graubert TA, DiPersio JF, Russell JH, Ley TJ. Perforin/granzymedependent and independent mechanisms are both important for the development of graft-versus-host disease after murine bone marrow transplantation. J Clin Invest 1997, Aug 15;100(4):904-11.

214. Merad M, Hoffmann P, Ranheim E, Slaymaker S, Manz MG, Lira SA, et al. Depletion of host langerhans cells before transplantation of donor alloreactive $\mathrm{T}$ cells prevents skin graft-versus-host disease. Nat Med 2004, May;10(5):510-7.

215. Li H, Kaplan DH, Matte-Martone C, Tan HS, Venkatesan S, Johnson K, et al. Langerhans cells are not required for graft-versus-host disease. Blood 2011, Jan 13;117(2):697-707.

216. Bennett CL, van Rijn E, Jung S, Inaba K, Steinman RM, Kapsenberg ML, Clausen BE. Inducible ablation of mouse langerhans cells diminishes but fails to abrogate contact hypersensitivity. J Cell Biol 2005, May 23;169(4):569-76.

217. Conlan TJ. Mechanisms of graft-versus-host-disease: A role for langerhans cells in regulating skin GVHD. London: University College London; 2014.

218. Antin JH. Acute graft-versus-host disease: Inflammation run amok? J Clin Invest 2001, Jun;107(12):1497-8.

219. Evert S. Chapter 7. Nutrition. In: Maziarz RT, Slater S, editors. Blood and marrow transplant handbook: comprehensive guide for patient care. Springer International Publishing; 2015. p. 81-9.

220. Ferrara JL, Deeg HJ. Graft-versus-Host disease. N Engl J Med 1991, Mar 7;324(10):667-74.

221. Shlomchik WD. Graft-versus-host disease. Nat Rev Immunol 2007, May;7(5):340-52.

222. McGill J, Legge KL. Cutting edge: Contribution of lung-resident $\mathrm{T}$ cell proliferation to the overall magnitude of the antigen-specific CD8 $T$ cell response in the lungs following murine influenza virus infection. J Immunol 2009, Oct 1;183(7):4177-81.

223. McGill J, Van Rooijen N, Legge KL. Protective influenza-specific CD8 T cell responses require interactions with dendritic cells in the lungs. J Exp Med 2008, Jul 7;205(7):1635-46.

224. Dolfi DV, Duttagupta PA, Boesteanu AC, Mueller YM, Oliai CH, Borowski $A B$, Katsikis PD. Dendritic cells and CD28 costimulation are required to 
sustain virus-specific CD8+ $T$ cell responses during the effector phase in vivo. J Immunol 2011, Apr;186(8):4599-608.

225. McLachlan JB, Catron DM, Moon JJ, Jenkins MK. Dendritic cell antigen presentation drives simultaneous cytokine production by effector and regulatory T cells in inflamed skin. Immunity 2009, Feb 20;30(2):277-88.

226. Eichler GS. Bioinformatics/biostatistics: Microarray analysis. Methods Mol Biol 2012;823:347-58.

227. Valafar F. Pattern recognition techniques in microarray data analysis: A survey. Ann N Y Acad Sci 2002, Dec;980:41-64.

228. Zhang B, Horvath S. A general framework for weighted gene coexpression network analysis. Stat Appl Genet Mol Biol 2005;4:Article17.

229. Langfelder $P$, Horvath S. WGCNA: An R package for weighted correlation network analysis. BMC Bioinformatics 2008;9:559.

230. Hu S, Zhou M, Jiang J, Wang J, Elashoff D, Gorr S, et al. Systems biology analysis of sjögren's syndrome and mucosa-associated lymphoid tissue lymphoma in parotid glands. Arthritis Rheum 2009, Jan;60(1):81-92.

231. Horvath S, Zhang B, Carlson M, Lu KV, Zhu S, Felciano RM, et al. Analysis of oncogenic signaling networks in glioblastoma identifies ASPM as a molecular target. Proc Natl Acad Sci U S A 2006, Nov 14;103(46):17402-7.

232. Matloubian M, Lo CG, Cinamon G, Lesneski MJ, Xu Y, Brinkmann V, et al. Lymphocyte egress from thymus and peripheral lymphoid organs is dependent on S1P receptor 1. Nature 2004, Jan 22;427(6972):355-60.

233. Kruisbeek AM. Isolation of mouse mononuclear cells. Curr Protoc Immunol 2001, May;Chapter 3:Unit 3.1.

234. Ema H, Morita Y, Nakauchi H, Matsuzaki Y. Isolation of murine hematopoietic stem cells and progenitor cells. Curr Protoc Immunol 2005, Jul;Chapter 22:Unit 22B.1.

235. McCarthy DA. Introduction to the general principles of sample preparation. In: Macey MG, editors. Cytometric analysis of cell phenotype and function. Cambridge University Press; 2001. p. 15-44.

236. Crispe IN. Isolation of mouse intrahepatic Iymphocytes. Curr Protoc Immunol 2001, May;Chapter 3:Unit 3.21.

237. Sheridan BS, Lefrançois L. Isolation of mouse lymphocytes from small intestine tissues. Curr Protoc Immunol 2012, Nov;Chapter 3:Unit3.19. 
238. Henri S, Poulin LF, Tamoutounour S, Ardouin L, Guilliams M, de Bovis B, et al. CD207+ CD103+ dermal dendritic cells cross-present keratinocytederived antigens irrespective of the presence of langerhans cells. J Exp Med 2010, Jan 18;207(1):189-206.

239. Holmes K, Lantz LM, Fowlkes BJ, Schmid I, Giorgi JV. Preparation of cells and reagents for flow cytometry. Curr Protoc Immunol 2001, Nov;Chapter 5:Unit 5.3.

240. Bolstad BM, Irizarry RA, Astrand M, Speed TP. A comparison of normalization methods for high density oligonucleotide array data based on variance and bias. Bioinformatics 2003, Jan 22;19(2):185-93.

241. Irizarry RA, Bolstad BM, Collin F, Cope LM, Hobbs B, Speed TP. Summaries of affymetrix genechip probe level data. Nucleic Acids Res 2003, Feb 15;31(4):e15.

242. Irizarry RA, Hobbs B, Collin F, Beazer-Barclay YD, Antonellis KJ, Scherf $U$, Speed TP. Exploration, normalization, and summaries of high density oligonucleotide array probe level data. Biostatistics 2003, Apr;4(2):24964.

243. Carvalho BS, Irizarry RA. A framework for oligonucleotide microarray preprocessing. Bioinformatics 2010, Oct 1;26(19):2363-7.

244. Johnson WE, Li C, Rabinovic A. Adjusting batch effects in microarray expression data using empirical bayes methods. Biostatistics 2007, Jan;8(1):118-27.

245. Leek JT, Johnson WE, Parker HS, Jaffe AE, Storey JD. The sva package for removing batch effects and other unwanted variation in highthroughput experiments. Bioinformatics 2012, Mar 15;28(6):882-3.

246. Reich M, Liefeld T, Gould J, Lerner J, Tamayo P, Mesirov JP. GenePattern 2.0. Nat Genet 2006, May;38(5):500-1.

247. Pan J-B, Hu S-C, Shi D, Cai M-C, Li Y-B, Zou Q, Ji Z-L. PaGenBase: A pattern gene database for the global and dynamic understanding of gene function. PLoS One 2013;8(12):e80747.

248. R Core Team (2016). [computer program]. R Foundation for Statistical Computing; Vienna, Austria.

249. Suzuki R, Shimodaira H. Pvclust: An R package for assessing the uncertainty in hierarchical clustering. Bioinformatics 2006, Jun 15;22(12):1540-2.

250. Suzuki R, Shimodaira H (2015). [computer program]. Japan. 
251. Gould J (2013). [computer program]. Broad Institute of MIT and Harvard; Cambridge, USA.

252. Ritchie ME, Phipson B, Wu D, Hu Y, Law CW, Shi W, Smyth GK. Limma powers differential expression analyses for rna-sequencing and microarray studies. Nucleic Acids Res 2015, Apr 20;43(7):e47.

253. Smyth GK. Linear models and empirical bayes methods for assessing differential expression in microarray experiments. Stat Appl Genet Mol Biol 2004;3:Article3.

254. Subramanian A, Tamayo P, Mootha VK, Mukherjee S, Ebert BL, Gillette $\mathrm{MA}$, et al. Gene set enrichment analysis: A knowledge-based approach for interpreting genome-wide expression profiles. Proc Natl Acad Sci U S A 2005, Oct 25;102(43):15545-50.

255. Subramanian A, Mesirov J, Tamayo P, Golub T, Lander E (2005). [computer program]. Broad Institute of MIT and Harvard; Cambridge, USA.

256. Du J, Yuan Z, Ma Z, Song J, Xie X, Chen Y. KEGG-PATH: Kyoto encyclopedia of genes and genomes-based pathway analysis using a path analysis model. Mol Biosyst 2014, Jul 29;10(9):2441-7.

257. Kanehisa M, Sato Y, Kawashima M, Furumichi M, Tanabe M. KEGG as a reference resource for gene and protein annotation. Nucleic Acids Res 2016, Jan 4;44(D1):D457-62.

258. Ashburner M, Ball CA, Blake JA, Botstein D, Butler H, Cherry JM, et al. Gene ontology: Tool for the unification of biology. The gene ontology consortium. Nat Genet 2000, May;25(1):25-9.

259. Gene Ontology Consortium. Gene ontology consortium: Going forward. Nucleic Acids Res 2015, Jan;43(Database issue):D1049-56.

260. Langfelder $P$, Horvath $S$. Fast $R$ functions for robust correlations and hierarchical clustering. J Stat Softw 2012, Mar;46(11).

261. Langfelder $P$, Luo R, Oldham MC, Horvath $S$. Is my network module preserved and reproducible? PLoS Comput Biol 2011;7(1):e1001057.

262. Shannon P, Markiel A, Ozier O, Baliga NS, Wang JT, Ramage D, et al. Cytoscape: A software environment for integrated models of biomolecular interaction networks. Genome Res 2003, Nov;13(11):2498-504.

263. Zhang B, Kirov S, Snoddy J. WebGestalt: An integrated system for exploring gene sets in various biological contexts. Nucleic Acids Res 2005, Jul 1;33(Web Server issue):W741-8. 
264. Wang J, Duncan D, Shi Z, Zhang B. WEB-based gene set analysis toolkit (webgestalt): Update 2013. Nucleic Acids Res 2013, Jul;41(Web Server issue):W77-83.

265. Barbie DA, Tamayo P, Boehm JS, Kim SY, Moody SE, Dunn IF, et al. Systematic RNA interference reveals that oncogenic kras-driven cancers require TBK1. Nature 2009, Nov 5;462(7269):108-12.

266. . [computer program]. GraphPad Software; USA.

267. Lamarthée B, Malard F, Gamonet C, Bossard C, Couturier M, Renauld J$\mathrm{C}$, et al. Donor interleukin-22 and host type I interferon signaling pathway participate in intestinal graft-versus-host disease via STAT1 activation and CXCL10. Mucosal Immunol 2016, Mar;9(2):309-21.

268. Shono Y, Docampo MD, Peled JU, Perobelli SM, Velardi E, Tsai JJ, et al. Increased gvhd-related mortality with broad-spectrum antibiotic use after allogeneic hematopoietic stem cell transplantation in human patients and mice. Sci Transl Med 2016, May 18;8(339):339ra71.

269. Wettstein PJ, Bailey DW. Immunodominance in the immune response to "multiple" histocompatibility antigens. Immunogenetics 1982;16(1):47-58.

270. Choi EY, Yoshimura Y, Christianson GJ, Sproule TJ, Malarkannan S, Shastri $\mathrm{N}$, et al. Quantitative analysis of the immune response to mouse non-mhc transplantation antigens in vivo: The $\mathrm{H} 60$ histocompatibility antigen dominates over all others. J Immunol 2001, Apr 1;166(7):4370-9.

271. Leung YF, Cavalieri D. Fundamentals of cdna microarray data analysis. Trends Genet 2003, Nov;19(11):649-59.

272. Pearson K. On lines and planes of closest fit to systems of points in space. Philosophical Magazine 1901;2(11):559-72.

273. Basilevsky AT. Statistical factor analysis and related methods: Theory and applications. New York, USA: John Wiley \& Sons; 1994.

274. Sokal RR, Michener CD. A statistical method for evaluating systematic relationships. University of Kansas Scientific Bulletin 1958;28:1409-38.

275. Berrar DP, Dubitzky W, Granzow M. A practical approach to microarray data analysis. Boston, MA: Kluwer Academic Publishers; 2003.

276. Ringnér M. What is principal component analysis? Nat Biotechnol 2008, Mar;26(3):303-4.

277. Nirmalakumari K, Harikumar R, Rajkumar P. Clustering techniques from significance analysis of microarrays. In: Senthilkumar M, Ramasamy V, Sheen S, Veeramani C, Bonato A, Batten L, editors. Computational 
Intelligence, Cyber Security and Computational Models: Proceedings of ICC3 2015. Singapore: Springer Singapore; 2016. p. 181-94.

278. Hennig C. Cluster-wise assessment of cluster stability. Computational Statistics \& Data Analysis 2007, Sep 15;52(1):258-71.

279. Lacher DA. Interpretation of laboratory results using multidimensional scaling and principal component analysis. Ann Clin Lab Sci 1987;17(6):412-7.

280. Bécavin C, Tchitchek N, Mintsa-Eya C, Lesne A, Benecke A. Improving the efficiency of multidimensional scaling in the analysis of highdimensional data using singular value decomposition. Bioinformatics 2011, May 15;27(10):1413-21.

281. Merico D, Isserlin R, Bader GD. Visualizing gene-set enrichment results using the cytoscape plug-in enrichment map. Methods Mol Biol 2011;781:257-77.

282. Wherry EJ, Ahmed R. Memory CD8 t-cell differentiation during viral infection. J Virol 2004, Jun;78(11):5535-45.

283. Wherry EJ. T cell exhaustion. Nat Immunol 2011, Jun;131(6):492-9.

284. Vasconcelos JR, Dominguez MR, Araújo AF, Ersching J, Tararam CA, Bruna-Romero O, Rodrigues MM. Relevance of long-lived CD8(+) T effector memory cells for protective immunity elicited by heterologous prime-boost vaccination. Front Immunol 2012;3:358.

285. Best JA, Blair DA, Knell J, Yang E, Mayya V, Doedens A, et al. Transcriptional insights into the CD8+ $T$ cell response to infection and memory T cell formation. Nat Immunol 2013, Feb 10.

286. Böttcher JP, Beyer M, Meissner F, Abdullah Z, Sander J, HÃđchst B, et al. Functional classification of memory CD8(+) $T$ cells by CX3CR1 expression. Nat Commun 2015;6:8306.

287. Gartlan KH, Markey KA, Varelias A, Bunting MD, Koyama M, Kuns RD, et al. Tc17 cells are a pro-inflammatory, plastic lineage of pathogenic CD8+ t-cells that induce GVHD without anti-leukemic effects. Blood 2015, Jul 23.

288. Gaublomme JT, Yosef N, Lee Y, Gertner RS, Yang LV, Wu C, et al. Single-Cell genomics unveils critical regulators of th17 cell pathogenicity. Cell 2015, Dec 3;163(6):1400-12. 
289. Stagg AJ, Kamm MA, Knight SC. Intestinal dendritic cells increase T cell expression of alpha4beta7 integrin. Eur J Immunol 2002, May;32(5):144554.

290. Johansson-Lindbom B, Svensson M, Pabst O, Palmqvist C, Marquez G, Förster R, Agace WW. Functional specialization of gut CD103+ dendritic cells in the regulation of tissue-selective T cell homing. J Exp Med 2005, Oct 17;202(8):1063-73.

291. Zimmerman ZF, Levy $\mathrm{RB}$. MiHA reactive CD4 and CD8 t-cells effect resistance to hematopoietic engraftment following reduced intensity conditioning. Am J Transplant 2006, Sep;6(9):2089-98.

292. Biburger M, Tiegs G. Animal models of autoimmune liver diseases. In: Gershwin ME, Vierling JM, Manns MP, editors. Liver Immunology: Principles and Practice. Totowa, NJ: Humana Press; 2007. p. 293-306.

293. Horvath S. Correlation and gene co-expression networks. In: Weighted network analysis: applications in genomics and systems biology. New York: Springer-Verlag; 2011.

294. Deshpande V, Sharma A, Mukhopadhyay R, Thota LNR, Ghatge M, Vangala RK, et al. Understanding the progression of atherosclerosis through gene profiling and co-expression network analysis in apob(tm2sgy)Idlr(tm1her) double knockout mice. Genomics 2016, Apr 28.

295. Barla A, Jurman G, Visintainer R, Squillario M, Filosi M, Riccadonna S, Furlanello $\mathrm{C}$. A machine learning pipeline for identification of discriminant pathways. In: Kasabov $\mathrm{N}$, editors. Springer Handbook of Bio/Neuroinformatics. Berlin, Heidelberg: Springer Berlin Heidelberg; 2014. p. 951-68.

296. Pappas DJ, Coppola G, Gabatto PA, Gao F, Geschwind DH, Oksenberg JR, Baranzini SE. Longitudinal system-based analysis of transcriptional responses to type I interferons. Physiol Genomics 2009, Aug 7;38(3):36271.

297. Novershtern N, Subramanian A, Lawton LN, Mak RH, Haining WN, McConkey $\mathrm{ME}$, et al. Densely interconnected transcriptional circuits control cell states in human hematopoiesis. Cell 2011, Jan 21;144(2):296309.

298. Furlan SN, Watkins B, Tkachev V, Flynn R, Cooley S, Ramakrishnan S, et al. Transcriptome analysis of GVHD reveals aurora kinase $A$ as a targetable pathway for disease prevention. Sci Transl Med 2015, Nov 25;7(315):315ra191. 
299. Leskovec JA, Rajaraman AA, Ullman JD. 11. Dimensionality reduction. In: Mining of massive datasets. Cambridge, UK: Cambridge University Press; 2014. p. 405-37.

300. Carlson MRJ, Zhang B, Fang Z, Mischel PS, Horvath S, Nelson SF. Gene connectivity, function, and sequence conservation: Predictions from modular yeast co-expression networks. BMC Genomics 2006;7:40.

301. Ghazalpour A, Doss S, Zhang B, Wang S, Plaisier C, Castellanos R, et al. Integrating genetic and network analysis to characterize genes related to mouse weight. PLoS Genet 2006, Aug 18;2(8):e130.

302. Fuller TF, Ghazalpour A, Aten JE, Drake TA, Lusis AJ, Horvath S. Weighted gene coexpression network analysis strategies applied to mouse weight. Mamm Genome 2007, Jul;18(6-7):463-72.

303. Emilsson V, Thorleifsson G, Zhang B, Leonardson AS, Zink F, Zhu J, et al. Genetics of gene expression and its effect on disease. Nature 2008, Mar 27;452(7186):423-8.

304. van Nas A, Guhathakurta D, Wang SS, Yehya N, Horvath S, Zhang B, et al. Elucidating the role of gonadal hormones in sexually dimorphic gene coexpression networks. Endocrinology 2009, Mar;150(3):1235-49.

305. Oldham MC, Horvath S, Geschwind DH. Conservation and evolution of gene coexpression networks in human and chimpanzee brains. Proc Natl Acad Sci U S A 2006, Nov 21;103(47):17973-8.

306. Miller JA, Oldham MC, Geschwind DH. A systems level analysis of transcriptional changes in alzheimer's disease and normal aging. $J$ Neurosci 2008, Feb 6;28(6):1410-20.

307. Oldham MC, Konopka G, Iwamoto K, Langfelder P, Kato T, Horvath S, Geschwind $\mathrm{DH}$. Functional organization of the transcriptome in human brain. Nat Neurosci 2008, Nov;11(11):1271-82.

308. Keller MP, Choi Y, Wang P, Davis DB, Rabaglia ME, Oler AT, et al. A gene expression network model of type 2 diabetes links cell cycle regulation in islets with diabetes susceptibility. Genome Res 2008, May;18(5):706-16.

309. Presson AP, Sobel EM, Papp JC, Suarez CJ, Whistler T, Rajeevan MS, et al. Integrated weighted gene co-expression network analysis with an application to chronic fatigue syndrome. BMC Syst Biol 2008;2:95. 
310. Weston DJ, Gunter LE, Rogers A, Wullschleger SD. Connecting genes, coexpression modules, and molecular signatures to environmental stress phenotypes in plants. BMC Syst Biol 2008;2:16.

311. Xue J, Schmidt SV, Sander J, Draffehn A, Krebs W, Quester I, et al. Transcriptome-based network analysis reveals a spectrum model of human macrophage activation. Immunity 2014, Feb 20;40(2):274-88.

312. Zaid A, Mackay LK, Rahimpour A, Braun A, Veldhoen M, Carbone FR, et al. Persistence of skin-resident memory T cells within an epidermal niche. Proc Natl Acad Sci U S A 2014, Apr 8;111(14):5307-12.

313. Pasparakis M, Haase I, Nestle FO. Mechanisms regulating skin immunity and inflammation. Nat Rev Immunol 2014, May;14(5):289-301.

314. Kupper TS, Fuhlbrigge RC. Immune surveillance in the skin: Mechanisms and clinical consequences. Nat Rev Immunol 2004, Mar;4(3):211-22.

315. Collin M, Jardine L. A question of persistence: Langerhans cells and graftversus-host disease. Exp Dermatol 2014, Apr;23(4):234-5.

316. Mielcarek M, Kirkorian AY, Hackman RC, Price J, Storer BE, Wood BL, et al. Langerhans cell homeostasis and turnover after nonmyeloablative and myeloablative allogeneic hematopoietic cell transplantation. Transplantation 2014, Sep 15;98(5):563-8.

317. Andani R, Robertson I, Macdonald KPA, Durrant S, Hill GR, Khosrotehrani K. Origin of langerhans cells in normal skin and chronic GVHD after hematopoietic stem-cell transplantation. Exp Dermatol 2014, Jan;23(1):75-7.

318. Ebens CL, Maillard I. Notch signaling in hematopoietic cell transplantation and T cell alloimmunity. Blood Rev 2013, Nov;27(6):269-77.

319. Tran IT, Sandy AR, Carulli AJ, Ebens C, Chung J, Shan GT, et al. Blockade of individual notch ligands and receptors controls graft-versushost disease. J Clin Invest 2013, Apr;123(4):1590-604.

320. Toubai T, Sun Y, Tawara I, Friedman A, Liu C, Evers R, et al. Ikaros-Notch axis in host hematopoietic cells regulates experimental graft-versus-host disease. Blood 2011, Jul 7;118(1):192-204.

321. Sandy AR, Chung J, Toubai T, Shan GT, Tran IT, Friedman A, et al. T cell-specific notch inhibition blocks graft-versus-host disease by inducing a hyporesponsive program in alloreactive CD4+ and CD8+ T cells. J Immunol 2013, Jun 1;190(11):5818-28. 
322. Kang SS, Herz J, Kim JV, Nayak D, Stewart-Hutchinson P, Dustin ML, McGavern DB. Migration of cytotoxic lymphocytes in cell cycle permits local MHC i-dependent control of division at sites of viral infection. J Exp Med 2011, Apr 11;208(4):747-59.

323. Poulin LF, Henri S, de Bovis B, Devilard E, Kissenpfennig A, Malissen B. The dermis contains langerin+ dendritic cells that develop and function independently of epidermal langerhans cells. J Exp Med 2007, Dec 24;204(13):3119-31.

324. Merad M, Ginhoux F, Collin M. Origin, homeostasis and function of langerhans cells and other langerin-expressing dendritic cells. Nat Rev Immunol 2008, Dec;8(12):935-47.

325. He T, Tang C, Xu S, Moyana T, Xiang J. Interferon gamma stimulates cellular maturation of dendritic cell line DC2.4 leading to induction of efficient cytotoxic $T$ cell responses and antitumor immunity. Cell Mol Immunol 2007, Apr;4(2):105-11.

326. Zhou F. Molecular mechanisms of ifn-gamma to up-regulate MHC class I antigen processing and presentation. Int Rev Immunol 2009;28(3-4):23960 .

327. Zhang Y, Sandy AR, Wang J, Radojcic V, Shan GT, Tran IT, et al. Notch signaling is a critical regulator of allogeneic CD4+ $t$-cell responses mediating graft-versus-host disease. Blood 2011, Jan 6;117(1):299-308.

328. Goulmy E, Schipper R, Pool J, Blokland E, Falkenburg JH, Vossen J, et al. Mismatches of minor histocompatibility antigens between hla-identical donors and recipients and the development of graft-versus-host disease after bone marrow transplantation. N Engl J Med 1996, Feb 1;334(5):2815.

329. Atkinson K, Farrell C, Chapman G, Downs K, Penny R, Biggs J. Female marrow donors increase the risk of acute graft-versus-host disease: Effect of donor age and parity and analysis of cell subpopulations in the donor marrow inoculum. Br J Haematol 1986, Jun;63(2):231-9.

330. Markiewicz M, Siekiera U, Karolczyk A, Szymszal J, Helbig G, Wojnar J, et al. Immunogenic disparities of 11 minor histocompatibility antigens (mhas) in hla-matched unrelated allogeneic hematopoietic SCT. Bone Marrow Transplant 2009, Feb;43(4):293-300.

331. Beilhack A, Schulz S, Baker J, Beilhack GF, Wieland CB, Herman El, et al. In vivo analyses of early events in acute graft-versus-host disease 
reveal sequential infiltration of t-cell subsets. Blood 2005, Aug 1;106(3):1113-22.

332. Silva IA, Olkiewicz K, Askew D, Fisher JM, Chaudhary MN, Vannella KM, et al. Secondary lymphoid organs contribute to, but are not required for the induction of graft-versus-host responses following allogeneic bone marrow transplantation: A shifting paradigm for $\mathrm{T}$ cell allo-activation. Biol Blood Marrow Transplant 2010, May;16(5):598-611.

333. Cocho L, Fernández I, Calonge M, Martínez V, González-García MJ, Caballero D, et al. Gene expression-based predictive models of graft versus host disease-associated dry eye. Invest Ophthalmol Vis Sci 2015, Jul;56(8):4570-81.

334. Buzzeo MP, Yang J, Casella G, Reddy V. A preliminary gene expression profile of acute graft-versus-host disease. Cell Transplant 2008;17(5):489-94.

335. Das H, Imoto S, Murayama T, Mizuno I, Sugimoto T, Taniguchi R, et al. Kinetic analysis of cytokine gene expression in patients with GVHD after donor lymphocyte infusion. Bone Marrow Transplant 2001, Feb;27(4):373-80.

336. Li L, Wang H, Kim JS, Pihan G, Boussiotis V. The cyclin dependent kinase inhibitor (R)-roscovitine prevents alloreactive $\mathrm{T}$ cell clonal expansion and protects against acute gvhd. Cell Cycle 2009, Jun 1;8(11):1794 - 1802.

337. Heidegger S, van den Brink MRM, Haas T, Poeck $H$. The role of patternrecognition receptors in graft-versus-host disease and graft-versusleukemia after allogeneic stem cell transplantation. Front Immunol 2014;5:337.

338. Byersdorfer CA, Tkachev V, Opipari AW, Goodell S, Swanson J, Sandquist $\mathrm{S}$, et al. Effector $\mathrm{T}$ cells require fatty acid metabolism during murine graft-versus-host disease. Blood 2013, Oct 31;122(18):3230-7.

339. Guedan S, Chen X, Madar A, Carpenito C, McGettigan SE, Frigault MJ, et al. ICOS-based chimeric antigen receptors program bipolar TH17/TH1 cells. Blood 2014, Aug 14;124(7):1070-80.

340. Yosef N, Shalek AK, Gaublomme JT, Jin H, Lee Y, Awasthi A, et al. Dynamic regulatory network controlling $\mathrm{TH} 17$ cell differentiation. Nature 2013, Apr 25;496(7446):461-8.

341. Ciofani M, Madar A, Galan C, Sellars M, Mace K, Pauli F, et al. A validated regulatory network for th17 cell specification. Cell 2012, Oct $12 ; 151(2): 289-303$. 
342. Angelini C, Costa V. Understanding gene regulatory mechanisms by integrating chip-seq and rna-seq data: Statistical solutions to biological problems. Front Cell Dev Biol 2014;2:51.

343. Matys V, Fricke E, Geffers R, Gössling E, Haubrock M, Hehl R, et al. TRANSFAC: Transcriptional regulation, from patterns to profiles. Nucleic Acids Res 2003, Jan 1;31(1):374-8.

344. Janky R, Verfaillie A, Imrichová $H$, Van de Sande B, Standaert L, Christiaens $V$, et al. IRegulon: From a gene list to a gene regulatory network using large motif and track collections. PLoS Comput Biol 2014, Jul;10(7):e1003731.

345. Romanoski CE, Glass CK, Stunnenberg HG, Wilson L, Almouzni G. Epigenomics: Roadmap for regulation. Nature 2015, Feb 19;518(7539):314-6.

346. Vargas-Díez E, García-Díez A, Marín A, Fernández-Herrera J. Lifethreatening graft-vs-host disease. Clin Dermatol 2005;23(3):285-300.

347. Vossen JM, Guiot HFL, Lankester AC, Vossen ACTM, Bredius RGM, Wolterbeek $\mathrm{R}$, et al. Complete suppression of the gut microbiome prevents acute graft-versus-host disease following allogeneic bone marrow transplantation. PLoS One 2014;9(9):e105706.

348. Fuller T, Langfelder P, Presson A, Horvath S. Review of weighted gene coexpression network analysis. In: Handbook of Statistical Bioinformatics. Springer; 2011. p. 369-88.

349. Scott-Boyer M-P, Haibe-Kains B, Deschepper CF. Network statistics of genetically-driven gene co-expression modules in mouse crosses. Front Genet 2013;4:291.

350. Mathewson N, Reddy P. The microbiome and graft versus host disease. Curr Stem Cell Rep 2015;1(1):39-47.

351. Kaplan DH, Anderson BE, McNiff JM, Jain D, Shlomchik MJ, Shlomchik WD. Target antigens determine graft-versus-host disease phenotype. J Immunol 2004;173(9):5467-75.

352. Mestas J, Hughes CC. Of mice and not men: Differences between mouse and human immunology. J Immunol 2004;172(5):2731-8. 


\section{Appendix A}

CD containing:

- .pdf files of the Supplementary Figures

- .xlsx files of the Suplementary Tables

\section{List of Supplementary Figures}

Supplementary Figure 1. Evaluation of internal reproducibility: correlation between the pairs of biological replicates for each tissue.

Supplementary Figure 2. Direct acyclic graph for enriched GO categories for module 1 genes.

Supplementary Figure 3. Direct acyclic graph for enriched GO categories for module 2 genes.

Supplementary Figure 4. Direct acyclic graph for enriched GO categories for module 3 genes. 
Supplementary Figure 5. Direct acyclic graph for enriched GO categories for module 4 genes.

Supplementary Figure 6. Direct acyclic graph for enriched GO categories for module 6 genes.

Supplementary Figure 7. Direct acyclic graph for enriched GO categories for module 7 genes.

Supplementary Figure 8. Direct acyclic graph for enriched GO categories for module 9 genes.

Supplementary Figure 9. Direct acyclic graph for enriched GO categories for module 13 genes.

Supplementary Figure 10. Direct acyclic graph for enriched GO categories for module 14 genes.

Supplementary Figure 11. Direct acyclic graph for enriched GO categories for module 15 genes.

Supplementary Figure 12. Direct acyclic graph for enriched GO categories for module 17 genes.

Supplementary Figure 13. Direct acyclic graph for enriched GO categories for module 23 genes.

Supplementary Figure 14. Direct acyclic graph for enriched GO categories for module 24 genes. 
Supplementary Figure 15. Direct acyclic graph for enriched GO categories for module 26 genes.

Supplementary Figure 16. Direct acyclic graph for enriched GO categories for module 27 genes.

Supplementary Figure 17. Direct acyclic graph for enriched GO categories for module 28 genes.

Supplementary Figure 18. Direct acyclic graph for enriched GO categories for module 29 genes.

Supplementary Figure 19. Direct acyclic graph for enriched GO categories for module 30 genes.

Supplementary Figure 20. Direct acyclic graph for enriched GO categories for module 31 genes.

Supplementary Figure 21. Direct acyclic graph for enriched GO categories for module 1 driver genes.

Supplementary Figure 22. Direct acyclic graph for enriched GO categories for module 2 driver genes.

Supplementary Figure 23. Direct acyclic graph for enriched GO categories for module 3 driver genes.

Supplementary Figure 24. Direct acyclic graph for enriched GO categories for module 4 driver genes. 
Supplementary Figure 25. Direct acyclic graph for enriched GO categories for module 6 driver genes.

Supplementary Figure 26. Direct acyclic graph for enriched GO categories for module 7 driver genes.

Supplementary Figure 27. Direct acyclic graph for enriched GO categories for module 9 driver genes.

Supplementary Figure 28. Direct acyclic graph for enriched GO categories for module 13 driver genes.

Supplementary Figure 29. Direct acyclic graph for enriched GO categories for module 14 driver genes.

Supplementary Figure 30. Direct acyclic graph for enriched GO categories for module 15 driver genes.

Supplementary Figure 31. Direct acyclic graph for enriched GO categories for module 17 driver genes.

Supplementary Figure 32. Direct acyclic graph for enriched GO categories for module 23 driver genes.

Supplementary Figure 33. Direct acyclic graph for enriched GO categories for module 24 driver genes.

Supplementary Figure 34. Direct acyclic graph for enriched GO categories for module 26 driver genes. 
Supplementary Figure 35. Direct acyclic graph for enriched GO categories for module 27 driver genes.

Supplementary Figure 36. Direct acyclic graph for enriched GO categories for module 28 driver genes.

Supplementary Figure 37. Direct acyclic graph for enriched GO categories for module 29 driver genes.

Supplementary Figure 38. Direct acyclic graph for enriched GO categories for module 30 driver genes.

Supplementary Figure 39. Direct acyclic graph for enriched GO categories for module 31 driver genes.

Supplementary Figure 40. Direct acyclic graph for enriched GO categories for the genes in module 28 that failed to be upregulated in the epidermis in the DT treated group.

\section{List of Supplementary Tables}

Supplementary Table 1. List of the liver, small intestine and skin specific genes excluded from the analysis.

Supplementary Table 2. List of overrepresented KEGG pathways in the 19 highly preserved modules. 
Supplementary Table 3. List of the driver genes for each of the 19 highly preserved modules.

Supplementary Table 4. List of KEGG pathways enriched in the dermis vs lymph nodes and in the epidermis vs dermis, in the presence (PBS) or absence (DT) of LC.

Supplementary Table 5. List of KEGG pathways overrepresented in the genes that failed to be up-regulated in the epidermis in the absence of LC. 


\section{Appendix B}

During my PhD, I contributed with experimental work that led to the publishing of four scientific articles:

- Verfuerth S, Santos e Sousa P, Beloki L, Murray M, Peters MD, Mackinnon S, Lowdell MW, Chakraverty R, Samuel ER. Generation of memory $\mathrm{T}$ cells for adoptive transfer using clinical-grade anti-cd62l magnetic beads. Bone Marrow Transplant 2015, Jun 15;50(10):1358-64.

- Veliça P, Zech M, Henson S, Holler A, Manzo T, Pike R, Santos e Sousa P, Zhang L, Schiedlmeier B, Pule M, Stauss H, Chakraverty R. Genetic regulation of fate decisions in therapeutic $T$ cells to enhance tumor protection and memory formation. Cancer Res 2015, Jul 1;75(13):264152.

- Sivakumaran S, Henderson S, Ward S, Santos e Sousa P, Manzo T, Zhang L, Conlan T, Means TK, D'Aveni M, Hermine O, Rubio MT, Chakraverty $\mathrm{R}$, Bennett $\mathrm{CL}$. Depletion of $\mathrm{CD} 11 \mathrm{c}(+)$ cells in the CD11c.DTR model drives expansion of unique CD64(+) Ly6c(+) monocytes that are poised to release TNF-a. Eur J Immunol 2015, Oct 14;46(1):192-203.

- D'Aveni M, Rossignol J, Coman T, Sivakumaran S, Henderson S, Manzo T, Santos e Sousa P, Bruneau J, Fouquet G, Zavala F, Alegria-Prévot O, Garfa-Traoré $M$, Suarez $F$, Trebeden-Nègre $H$, Mohty $M$, Bennett $C L$, Chakraverty R, Hermine O, Rubio MT. G-CSF mobilizes CD34+ regulatory monocytes that inhibit graft-versus-host disease. Sci Transl Med 2015, Apr 1;7(281):281ra42. 